\title{
AFIP-2 Irradiation Summary Report
}

D. M. Perez

M. A. Lillo

G. S. Chang

G. A. Roth

N. E. Woolstenhulme

D. M. Wachs

April 2011

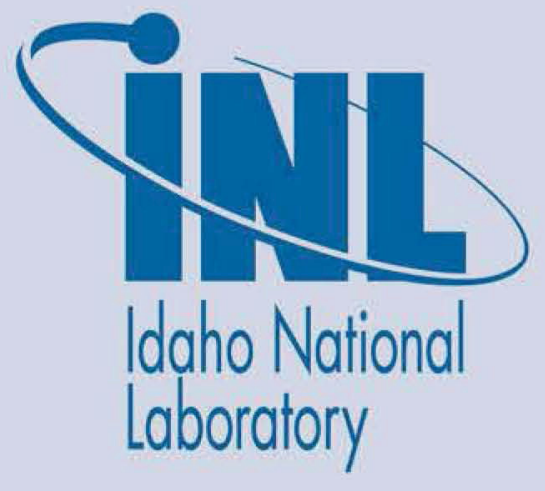

The INL is a U.S. Department of Energy National Laboratory operated by Battelle Energy Alliance 


\section{DIS CLAIMER}

This information was prepared as an account of work sponsored by an agency of the U.S. Government. Neither the U.S. Government nor any agency thereof, nor any of their employees, makes any warranty, expressed or implied, or assumes any legal liability or responsibility for the accuracy, completeness, or usefulness, of any information, apparatus, product, or process disclosed, or represents that its use would not infringe privately owned rights. References herein to any specific commercial product, process, or service by trade name, trade mark, manufacturer, or otherwise, does not necessarily constitute or imply its endorsement, recommendation, or favoring by the U.S. Government or any agency thereof. The views and opinions of authors expressed herein do not necessarily state or reflect those of the U.S. Government or any agency thereof. 


\section{AFIP-2 Irradiation Summary Report}

D. M. Perez, M. A. Lillo, G. S. Chang,

G. A. Roth, N. E. Woolstenhulme,

D. M. Wachs

April 2011

Idaho National Laboratory Idaho Falls, Idaho 83415

http://www.inl.gov

Prepared for the

U.S. Department of Energy

Office of

National Nuclear Security Administration

Under DOE Idaho Operations Office

Contract DE-AC07-05ID14517 


\title{
AFIP-2 Irradiation Summary Report
}

\author{
INL/EXT-11-21599
}

April 2011
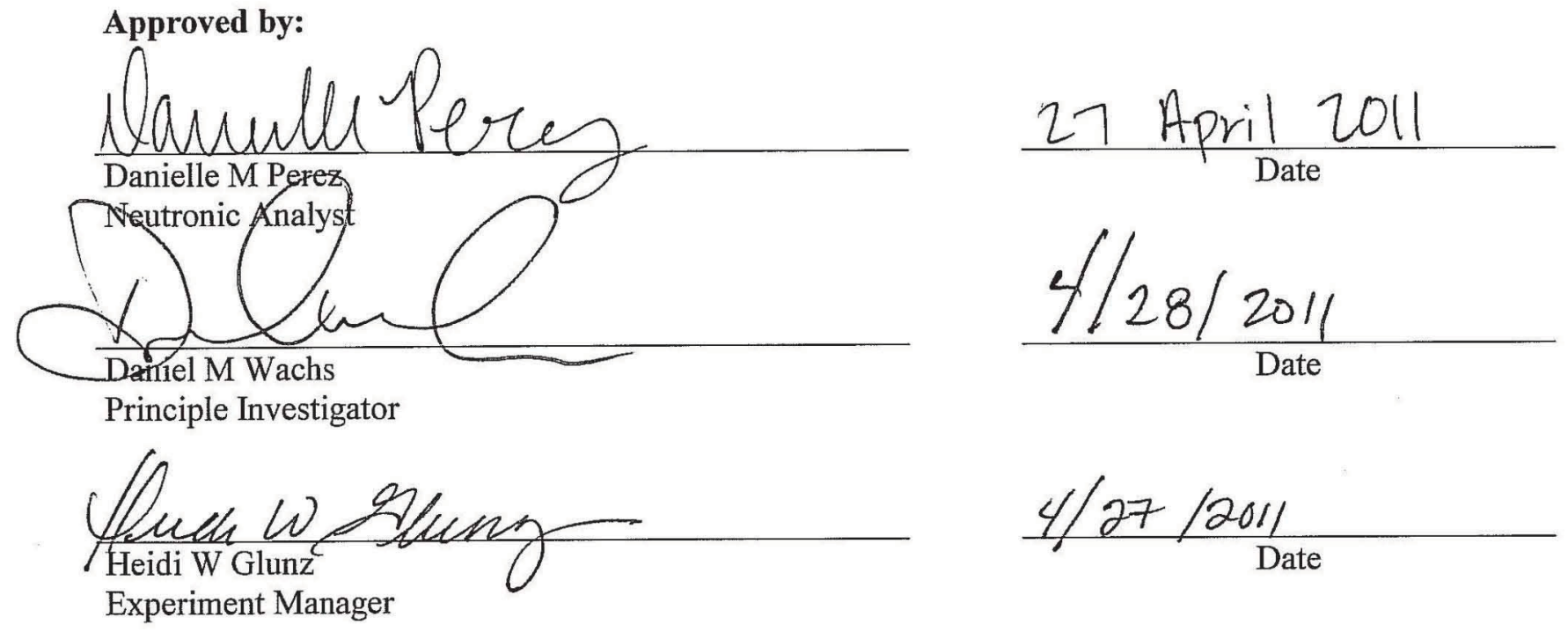


\section{SUMMARY}

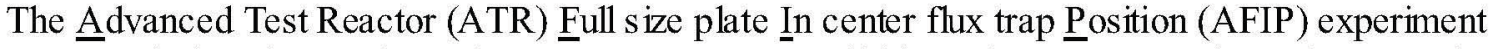
AFIP-2 was designed to evaluate the performance of monolithic fuels at a prototypic scale of 2.25 inches $x 21.5$ inches $x 0.050$ inches $(5.75 \mathrm{~cm}$ x $54.6 \mathrm{~cm}$ x $0.13 \mathrm{~cm})$. The AFIP-2 experiment was fabricated by friction bond (FB) and consists of two plates, one with a zirconium ( $\mathrm{Zr}$ ) diffusion barrier and one with a silicon (Si) enhanced fuel/clad interface ${ }^{1,2}$.

The following report summarizes the life of the AFIP-2 experiment through end of irradiation, including a brief description of the safety analysis, as-run neutronic analysis results, hydraulic testing results, and thermal analys is results. The safety analyses performed for AFIP-2 are summarized in Table 5 of the follow ing report. 


\section{CONTENTS}

SUMMARY.

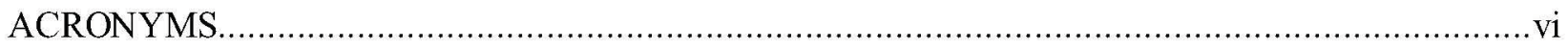

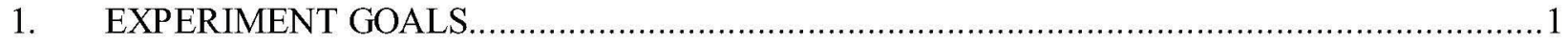

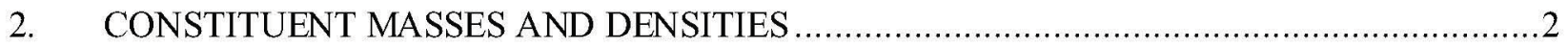

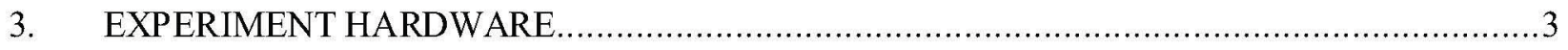

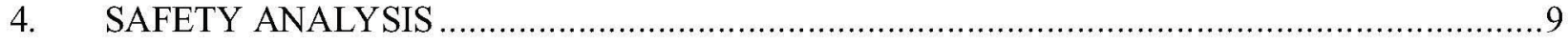

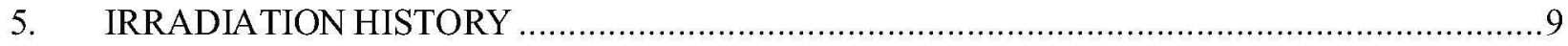

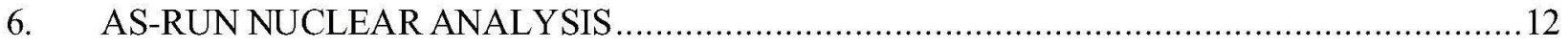

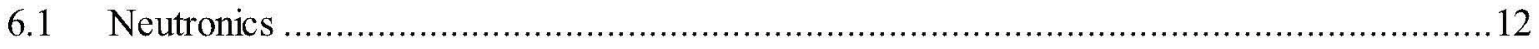

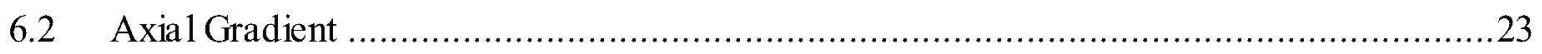

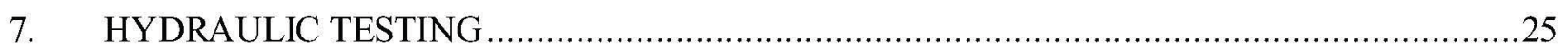

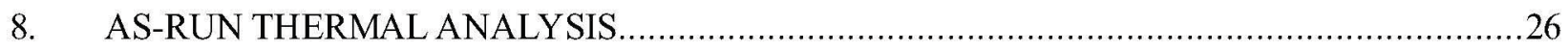

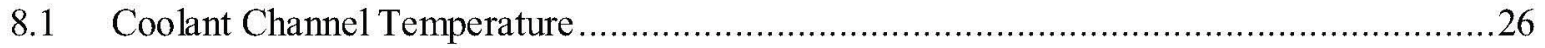

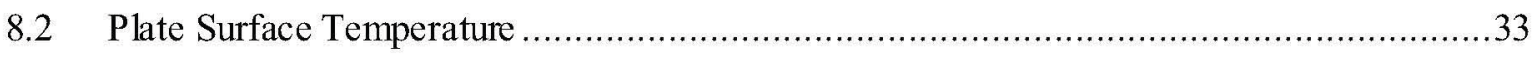

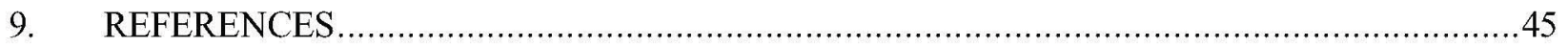

Appendix A Individua1 Plate Power and Fission Density Plots ...................................................46

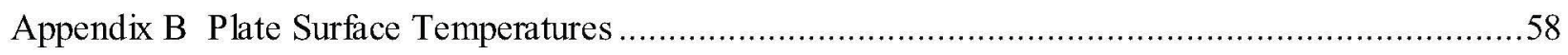

\section{FIGURES}

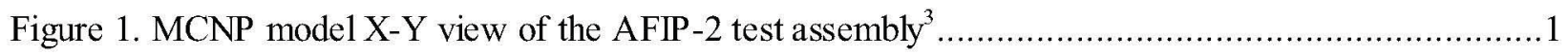

Figure 2. DWG 635793 ATR Full Size Plate in Center Flux Trap Position (AFIP) Test Train

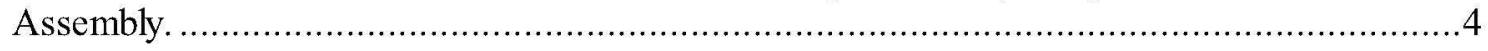

Figure 3. Isometric view of the AFIP-2 Test Train Assembly..............................................

Figure 4. DWG-635789 REV 4 AFIP-2 Frame Assembly.......................................................

Figure 5. DWG-635788 REV 2 ATR Full Size Plate in Center Flux Trap Position Fuel Plate. ...............7

Figure 6. Radial Cross Section of the AFIP Test Train Assembly .................................................

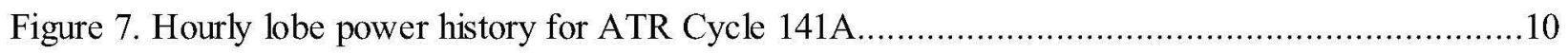

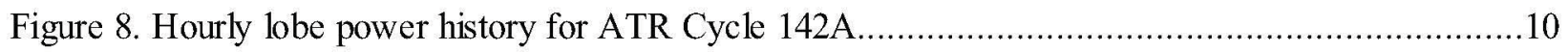

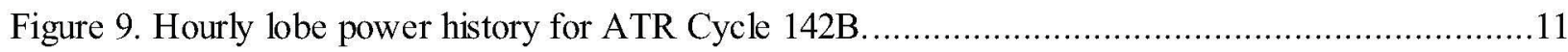


Figure 10. AFIP-2 axial neutron flux profile. ${ }^{3}$ .23

Figure 11. AFIP-2 corrected fission density axial profile.

Figure 12. Coolant channel temperatures as a function of location along the test assembly at BOC 141A (0.0 EFPD).

Figure 13. Coolant channel temperatures as a function of location along the test assembly at MOC 141A (15.0 EFPD).

Figure 14. Coolant channel temperatures as a function of location along the test assembly at MOC 141A (25.0 EFPD).

Figure 15. Coolant channel temperatures as a function of location along the test assembly at EOC 141A (32.4 EFPD)

Figure 16. Coolant channel temperatures as a function of location along the test assembly at BOC 142A (32.4 EFPD)

Figure 17. Coolant channel temperatures as a function of location along the test assembly at MOC 142A (48.4 EFPD).

Figure 18. Coolant channel temperatures as a function of location along the test assembly at MOC 142A (64.4 EFPD)

Figure 19. Coolant channel temperatures as a function of location along the test assembly at EOC 142A (80.4 EFPD)

Figure 20. Coolant channel temperatures as a function of location along the test assembly at BOC 142B (80.4 EFPD). NOTE: AFIP-1 was inserted into plate position B and BOC 142B.

Figure 21. Coolant channel temperatures as a function of location along the test assembly at MOC 142B (96.4 EFPD).

Figure 22. Coolant channel temperatures as a function of location along the test assembly at MOC 142B (110.1 EFPD).

Figure 23. Coolant channel temperatures as a function of location along the test assembly at EOC 142B (132.4 EFPD).

\section{TABLES}

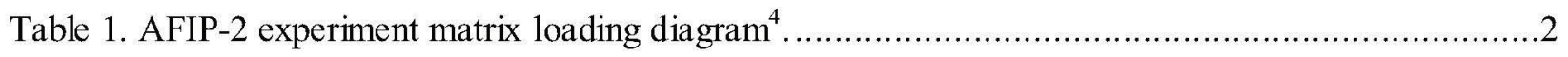

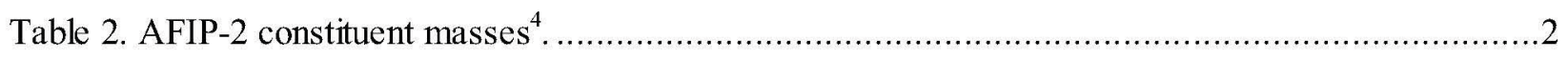

Table 4. AFIP-2 Irradiation Hardware Drawing List................................................................

Table 5. Summary table of the safety analyses done for the AFIP-2 experiment. ..............................

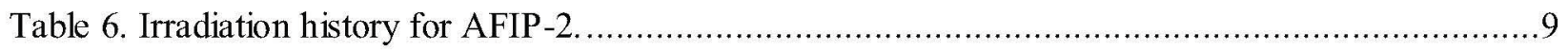

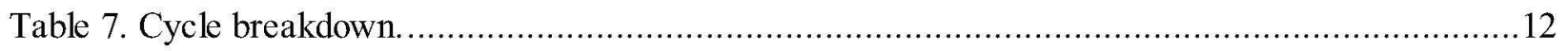

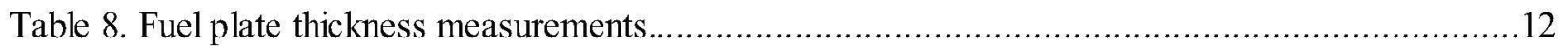

Table 9. End of cycle fission power density, surface heat flux, U-235 depletion and fission density for AFIP-2 experiment.

Table 10. Cycle 141A, MCNP-Calculated heat generation rate and neutron flux for AFIP-2 fuel foils 0 EFPD (BOC) Center Lobe Power at 23.4 MW. ${ }^{3}$ 
Table 11. Cycle 141A, MCNP-Calculated HGR, Neutron Flux, Depletion, and Fiss ion Density for AFIP-2 Fuel Foils, 15.0 EFPD (MOC1), Center Lobe Power@23.4 MW (15.0 EFPD Cumulative). ${ }^{3}$

Table 12. Cycle 141A, MCNP-Calculated HGR, Neutron Flux, Depletion, and Fission Density for AFIP-2 Fuel Foils, 25.0 EFPD (MOC2), Center Lobe Power@23.4 MW (25.0 EFPD Cumulative). ${ }^{3}$

Table 14. Cycle 142A, MCNP-Calculated HGR and Neutron Flux for AFIP-2 Fuel Foils, 0 EFPD (BOC), Center Lobe Power@24.7 MW.3... 16

Table 15. Cycle 142A, MCNP-Calculated HGR, Neutron Flux, Depletion, and Fission Density for AFIP-2 Fuel Foils, 16.0 EFPD (MOC1), Center Lobe Power@24.7 MW (48.4 EFPD Cumulative). ${ }^{3}$...... 16

Table 16. Cycle 142A, MCNP-Calculated HGR, Neutron Flux, Depletion, and Fission Density for AFIP-2 Fuel Foils, 32.0 EFPD (MOC2), Center Lobe Power@ 24.7 MW (64.4 EFPD Cumulative) ${ }^{3}$.

Table 17 Cycle 142A, MCNP-Calculated HGR, Neutron Flux, Depletion, and Fission Density for AFIP-2 Fuel Foils, 48.0 EFPD (EOC), Center Lobe Power@ 24.7 MW (80.4 EFPD Cumulative) ${ }^{3}$.

Table 18 Cycle 142B, MCNP-Calculated HGR and Neutron Flux for AFIP-2 Fuel Foils, 0 EFPD (BOC), Center Lobe Power@25.4 MW.3.

Table 19 Cycle 142B, MCNP-Calculated HGR, Neutron Flux, Depletion, and Fission Density for AFIP-2 Fuel Foils, 16.0 EFPD (MOC1), Center Lobe Power@ 25.4 MW (96.4 EFPD Cumulative) ${ }^{3}$.....

Table 20 Cycle 142B, MCNP-Calculated HGR, Neutron Flux, Depletion, and Fission Density for AFIP-2 Fuel Foils, 29.7 EFPD (MOC2), Center Lobe Power@25.4 MW (110.1 EFPD Cumulative) ${ }^{3}$.

Table 21 Cycle 142B, MCNP-Calculated HGR, Neutron Flux, Depletion, and Fission Density for AFIP-2 Fuel Foils, 52.0 EFPD (EOC), Center Lobe Power@ 25.4 MW (132.4 EFPD Cumulative) ${ }^{3}$

Table 22. Corrected fission power density (W/cc) and fission density (fissions/cc) for ATR Cycle $141 \mathrm{~A}$.

Table 23. Corrected fission power density (W/cc) and fission dens ity (fissions/cc) for ATR Cycle $142 \mathrm{~A}$.

Table 24. Corrected fission power density (W/cc) and fission dens ity (fissions/cc) for ATR Cycle 142B.

Table 25. AFIP irradiation vehicle flow conditions for each orifice configuration. ${ }^{7}$.............................25

Table 26. Temperature $\left({ }^{\circ} \mathrm{C}\right)$ map of the east side of plate 2TT at EOC 141A (32.4 EFPD)...................33

Table 27. Temperature $\left({ }^{\circ} \mathrm{C}\right)$ map of the west side of plate 2TT at EOC 141A (32.4 EFPD).................34

Table 28. Temperature $\left({ }^{\circ} \mathrm{C}\right)$ map of the east side of plate $2 \mathrm{BZ}$ at EOC 141A (32.4 EFPD)..................35

Table 29. Temperature $\left({ }^{\circ} \mathrm{C}\right)$ map of the west side of plate $2 \mathrm{BZ}$ at EOC 141A (32.4 EFPD).................36

Table 30. Temperature $\left({ }^{\circ} \mathrm{C}\right)$ map of the east side of plate 2TT at EOC 142A (80.4 EFPD)..................37

Table 31. Temperature $\left({ }^{\circ} \mathrm{C}\right)$ map of the west side of plate 2TT at EOC 142A (80.4 EFPD).................38 
Table 32. Temperature $\left({ }^{\circ} \mathrm{C}\right)$ map of the east side of plate 2BZ at EOC 142A (80.4 EFPD) ..................39

Table 33. Temperature $\left({ }^{\circ} \mathrm{C}\right)$ map of the west side of plate 2BZ at EOC 142A (80.4 EFPD)................40

Table 34. Temperature $\left({ }^{\circ} \mathrm{C}\right)$ map of the east side of plate 2TT at EOC 142B (132.4 EFPD)................41

Table 35. Temperature $\left({ }^{\circ} \mathrm{C}\right)$ map of the west side of plate 2TT at EOC 142B (132.4 EFPD)...............42

Table 36. Temperature $\left({ }^{\circ} \mathrm{C}\right)$ map of the east side of plate 2BZ at EOC 142B (132.4 EFPD)................43

Table 37. Temperature $\left({ }^{\circ} \mathrm{C}\right)$ map of the west side of plate $2 \mathrm{BZ}$ at EOC 142B (132.4 EFPD) ...............44 


\section{ACRONYMS}

\begin{tabular}{ll} 
Al & Aluminum \\
ATR & Advanced Test Reactor \\
AFIP & ATR Eull-size plate In center flux trap Position \\
CFT & Center Flux Trap \\
DAS & Data Acquisition System \\
DNBR & Departure from Nucleate Boiling Ratio \\
ECAR & Engineering Calculations and Analysis Report \\
EFPD & Effective Full Power Days \\
FB & Friction Bond \\
FD & Fuel Development \\
FIR & Flow Instability Ratio \\
GTRI & Global Threat Reduction Initiative \\
MCNP & Monte Carlo N-Particle \\
Mo & Molybdenum \\
RERTR & Reduced Enrichment Research and Test Reactor \\
Si & Silicon \\
U & Uranium \\
U-Mo & Uranium-Molybdenum \\
Zr & Zirconium \\
\hline
\end{tabular}




\section{AFIP-2 Irradiation Summary Report}

\section{EXPERIMENT GOALS}

In support of the Global Threat Reduction Initiative (GTRI) Fuel Development (FD) program (historically known as Reduced Enrichment for Research and Test Reactors (RERTR)), the Advanced Test Reactor (ATR) Full size plate In center flux trap Position (AFIP) experiment AFIP-2 was designed to evaluate the performance of monolithic fuels at a prototypic scale of 2.25 inches $\times 21.5$ inches $\times 0.050$ inches $(5.75 \mathrm{~cm} \times 54.6 \mathrm{~cm} \times 0.13 \mathrm{~cm})$. The AFIP-2 experiment was fabricated by friction bond $(\mathrm{FB})$ and consists of two plates, one with a zirconium ( $\mathrm{Zr}$ ) diffusion barrier and one with a silicon ( $\mathrm{Si}$ ) enhanced fuel/clad interface ${ }^{1,2}$.

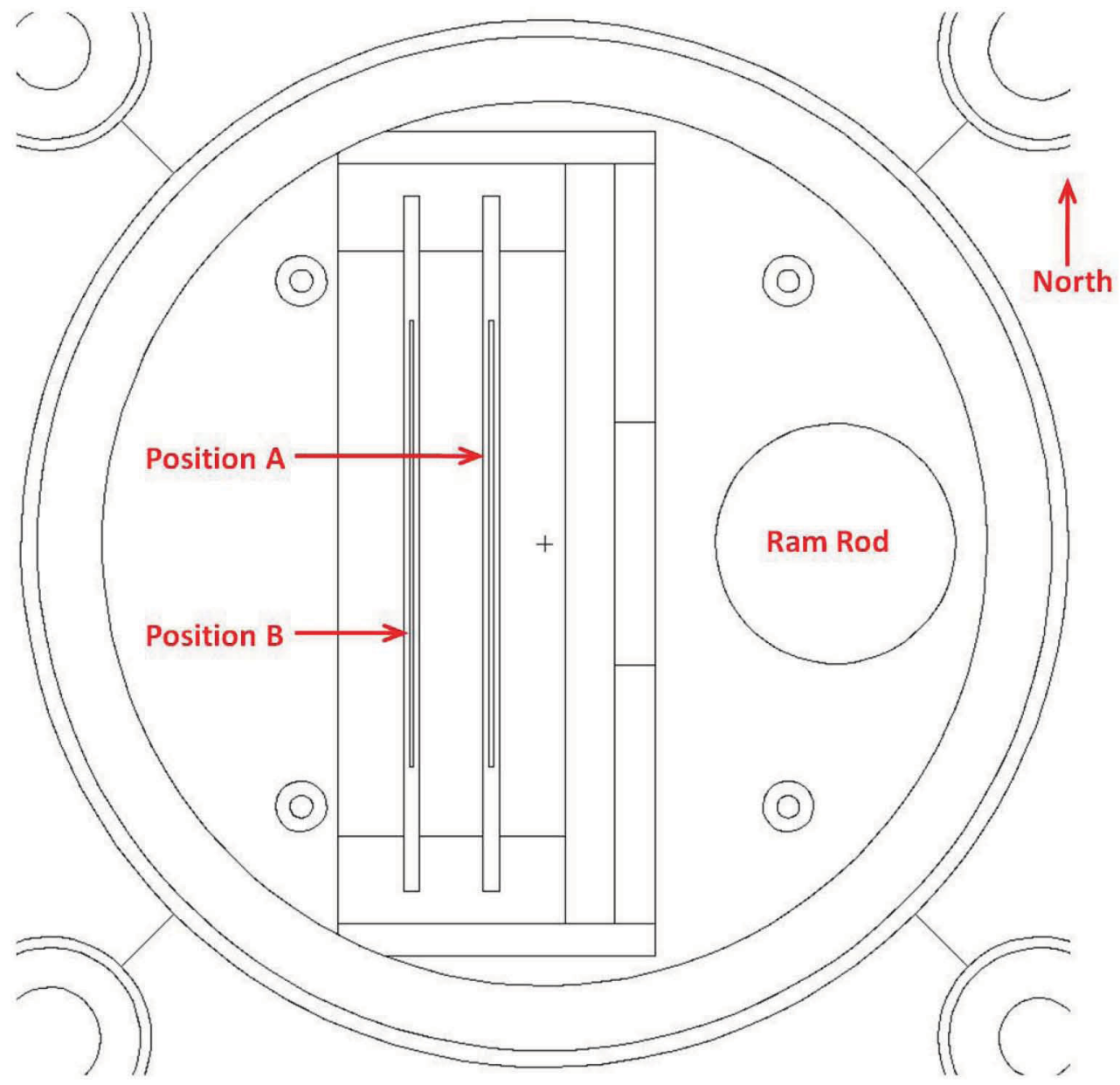

Figure 1. MCNP model X-Y view of the AFIP-2 test assembly ${ }^{3}$.

The AFIP-2 test assembly holds two full length (48 inch) frames, designated as A and B, a ramrod and four flux monitor wires. Frame A is located closest to the ramrod and frame B is on the outer (west) location in the assembly (see Figure 1). Each fuel plate is nominally 22.5 inches in length, 2.205 inches in width and 0.050 inches in thickness. The nominal fuel meat zone is 20.625 inches in length and 1.450 inches in width. The AFIP-2 experiment consisted of two fuel plates that were located in the A position of the test assembly with a plate on the top half of the frame and a plate on the bottom half. The experiment matrix is shown in Table 1. 
Table 1. AFIP-2 experiment matrix loading diagram ${ }^{4}$.

\begin{tabular}{|c|c|c|}
\hline $\begin{array}{c}\text { Asse mbly } \\
\text { Position }\end{array}$ & A-Position & B-Position \\
\hline \multirow{2}{*}{ Top } & $\begin{array}{c}\text { U-10 Mo } \\
\text { Thermal Spray Si } \\
\text { Friction Bond } \\
\text { 2TT }\end{array}$ & $\begin{array}{c}\text { Blank } \\
\text { Al-6061 }\end{array}$ \\
\hline & U-10 Mo & BT-Dum \\
\hline Bottom & Zirconium & Blank \\
& $\begin{array}{c}\text { Friction Bond } \\
\text { 2BZ }\end{array}$ & Al-6061 \\
& \multicolumn{2}{|}{ BB-Dum } \\
\hline
\end{tabular}

\section{CONSTITUENT MASSES AND DENSITIES}

The constituent masses and densities for plate $2 \mathrm{TT}$ and $2 \mathrm{BZ}$ were obtained from the as-built data package ${ }^{4}$ plate summary sheets. Table 2 summarizes the constituent masses for both plates and Table 3 summarizes the constituent densities for both plates.

Table 2. AFIP-2 constituent masses ${ }^{4}$.

\begin{tabular}{|c|c|c|c|c|c|c|}
\hline $\begin{array}{c}\text { Fuel Plate } \\
\text { Location }\end{array}$ & $\begin{array}{c}\text { Fuel } \\
\text { Plate ID }\end{array}$ & $\begin{array}{c}\text { Total-U } \\
(\mathrm{g})\end{array}$ & $\begin{array}{c}\mathrm{U}-238 \\
(\mathrm{~g})\end{array}$ & $\begin{array}{c}\mathrm{U}-235 \\
(\mathrm{~g})\end{array}$ & $\begin{array}{c}\mathrm{Zr} \\
(\mathrm{g})\end{array}$ & $\begin{array}{c}\text { Mo } \\
(\mathrm{g})\end{array}$ \\
\hline Top & $2 \mathrm{TT}$ & 97.955 & 78.481 & 19.474 & -- & 11.231 \\
\hline Bottom & $2 \mathrm{BZ}$ & 89.580 & 71.771 & 17.809 & 8.860 & 10.271 \\
\hline
\end{tabular}

Table 3. AFIP-2 constituent densities.

\begin{tabular}{|c|c|c|c|c|c|c|c|}
\hline $\begin{array}{c}\text { Fuel Plate } \\
\text { Location }\end{array}$ & $\begin{array}{c}\text { Fuel Plate } \\
\text { ID }\end{array}$ & $\begin{array}{c}\text { Fuel Meat } \\
\text { Volume } \\
(\mathrm{cc})\end{array}$ & $\begin{array}{c}\text { Total-U } \\
(\mathrm{g} / \mathrm{cc})\end{array}$ & $\begin{array}{c}\mathrm{U}-238 \\
(\mathrm{~g} / \mathrm{cc})\end{array}$ & $\begin{array}{c}\mathrm{U}-235 \\
(\mathrm{G} / \mathrm{cc})\end{array}$ & $\begin{array}{c}\mathrm{Zr} \\
(\mathrm{g} / \mathrm{cc})\end{array}$ & $\begin{array}{c}\text { Mo } \\
(\mathrm{g} / \mathrm{cc})\end{array}$ \\
\hline Top & 2TT & 6.445 & 15.198 & 12.176 & 3.021 & -- & 1.742 \\
\hline Bottom & $2 \mathrm{BZ}$ & 6.783 & 13.898 & 11.135 & 2.763 & 1.375 & 1.594 \\
\hline
\end{tabular}




\section{EXPERIMENT HARDWARE}

The experiment hardware list for AFIP-2 is that used for all of the four-plate AFIP experiments ${ }^{5}$. The drawing numbers and titles are in Table 4.

Table 4. AFIP-2 Irradiation Hardware Drawing List.

\begin{tabular}{|c|c|}
\hline $\begin{array}{l}\text { Drawing } \\
\text { Number }\end{array}$ & Drawing Title \\
\hline 635791 & $\begin{array}{l}\text { ATR Full Size Plate in Center Flux Trap Position (AFIP) Flux Monitor } \\
\text { Wire Holder Assembly Details }\end{array}$ \\
\hline 635792 & $\begin{array}{l}\text { ATR Full Size Plate in Center Flux Trap Position (AFIP) Plate Holder } \\
\text { Tube Assembly and Details }\end{array}$ \\
\hline 635793 & $\begin{array}{l}\text { ATR Full Size Plate in Center Flux Trap Position (AFIP) Test Train } \\
\text { Assembly }\end{array}$ \\
\hline 635790 & $\begin{array}{l}\text { ATR Full Size Plate in Center Flux Trap Position (AFIP) Ramrod and } \\
\text { Ram Details }\end{array}$ \\
\hline 636544 & $\begin{array}{l}\text { ATR Full Size Plate in Center Flux Trap Position (AFIP) Holder } \\
\text { Retriever Assembly and Details }\end{array}$ \\
\hline 635789 & $\begin{array}{l}\text { RERTR ATR Full Size Plate in Center Flux Trap Position Fue1 Plate } \\
\text { Frame Assembly and Details }\end{array}$ \\
\hline 635788 & RERTR ATR Full Size Plate in Center Flux Trap Position Fue1 Plate \\
\hline
\end{tabular}

The AFIP test train assembly as shown in Figure 2 shows the main components of the test assembly, which includes the flux monitor wire holders, the ram and ramrod, the frame assembly and the fuel plates. Figure 3 shows the test train assembly with the retriever attached to the top. The retriever is used to get the test train assembly out of the reactor. The flux monitor wire holders house the flux monitor wires. The ram is used to clamp onto the rails of the fuel plate frame assemblies to minimize vibrations and hold the fuel plate frame assemblies in place. The ramrod is the last component to be inserted; it is used to push the ram against the fuel plate frame assembly. The frame assembly holds the fuel plates into place and is shown in Figure 4. Figure 5 has the specific fuel plate dimensions and nominal fuel foil dimensions. Figure 6 is a radial cross section of the test train assembly and shows the locations of all the components. 


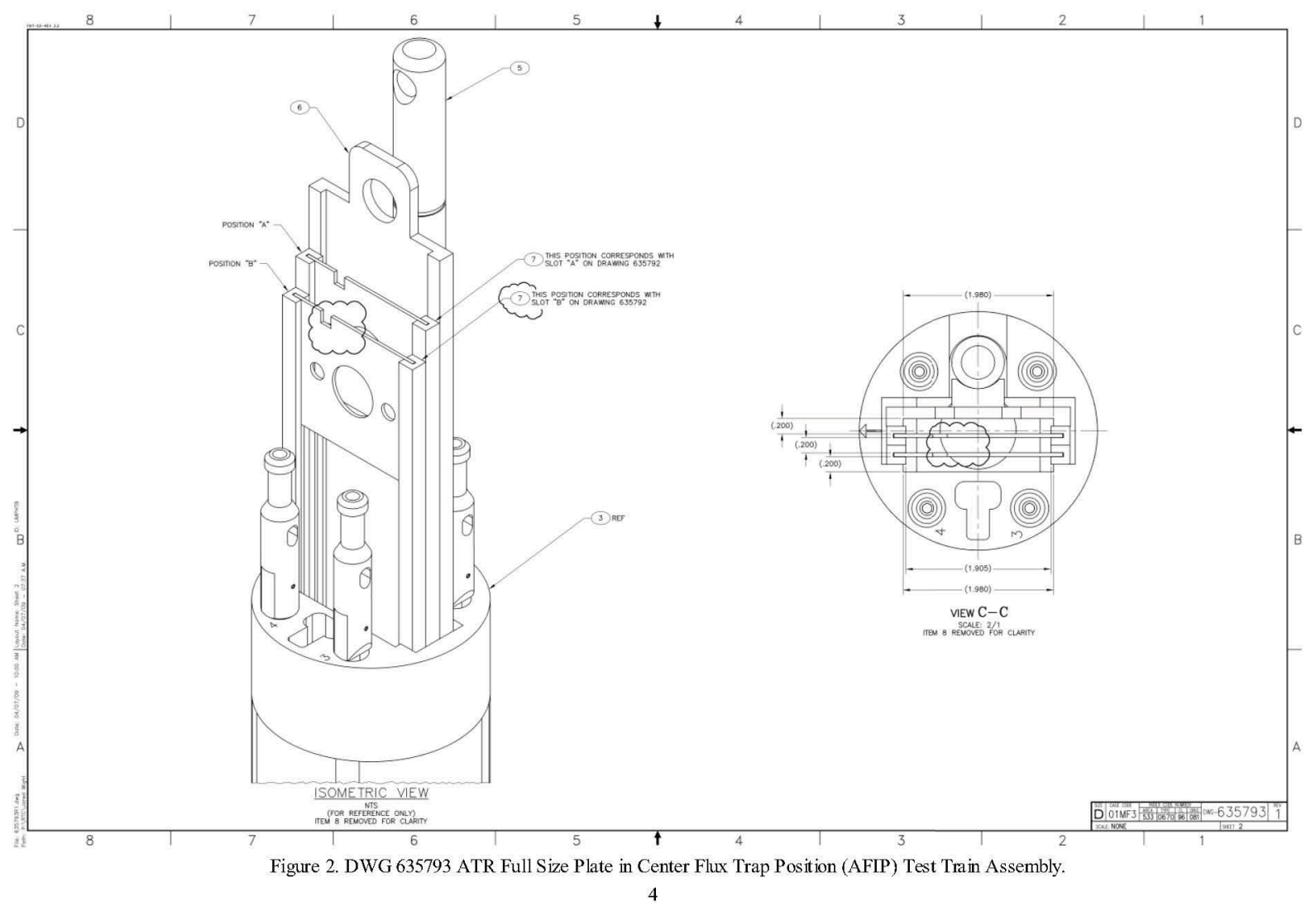




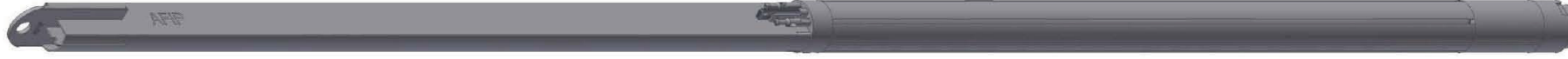

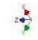

Figure 3. Isometric view of the AFIP-2 Test Train Assembly. 


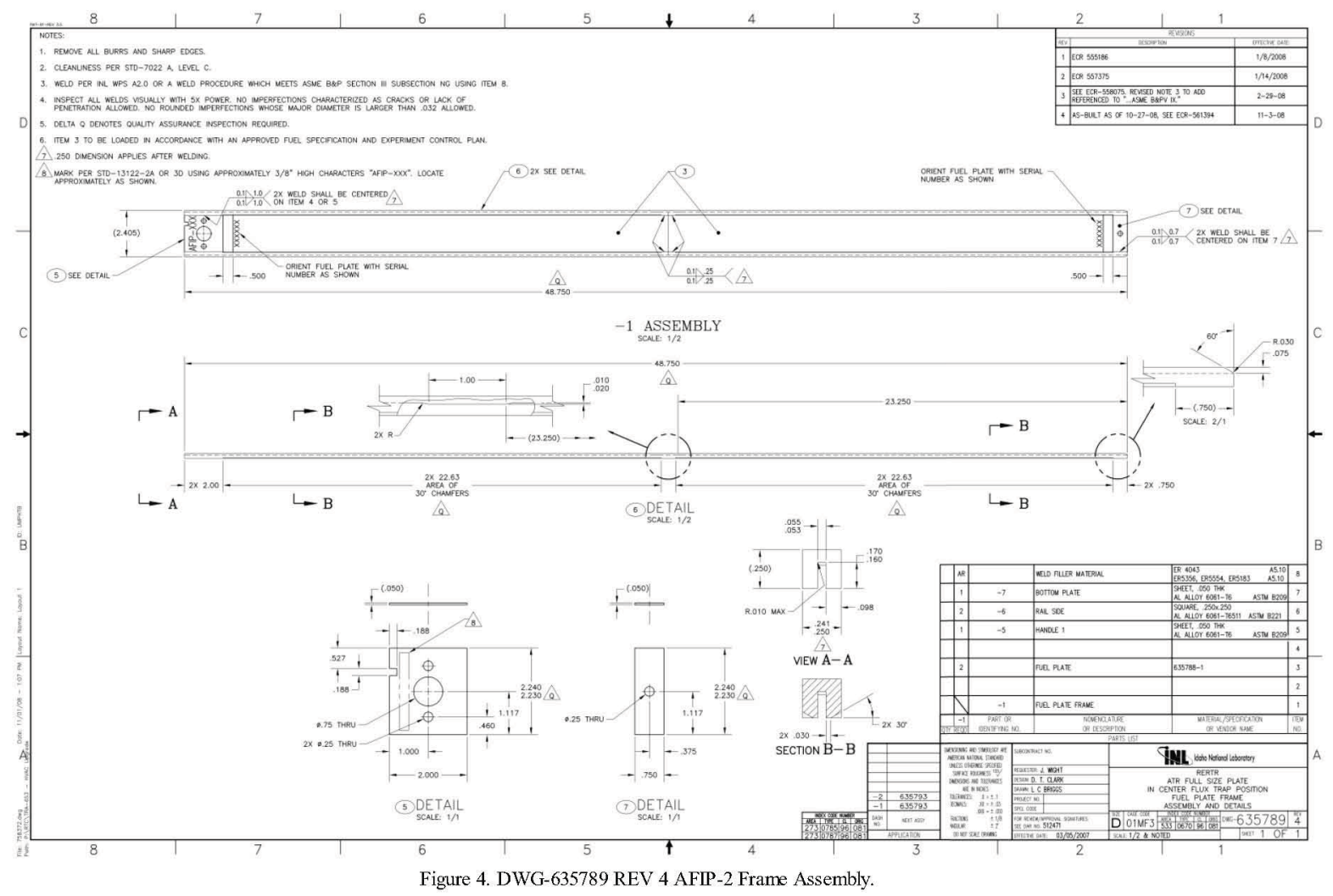




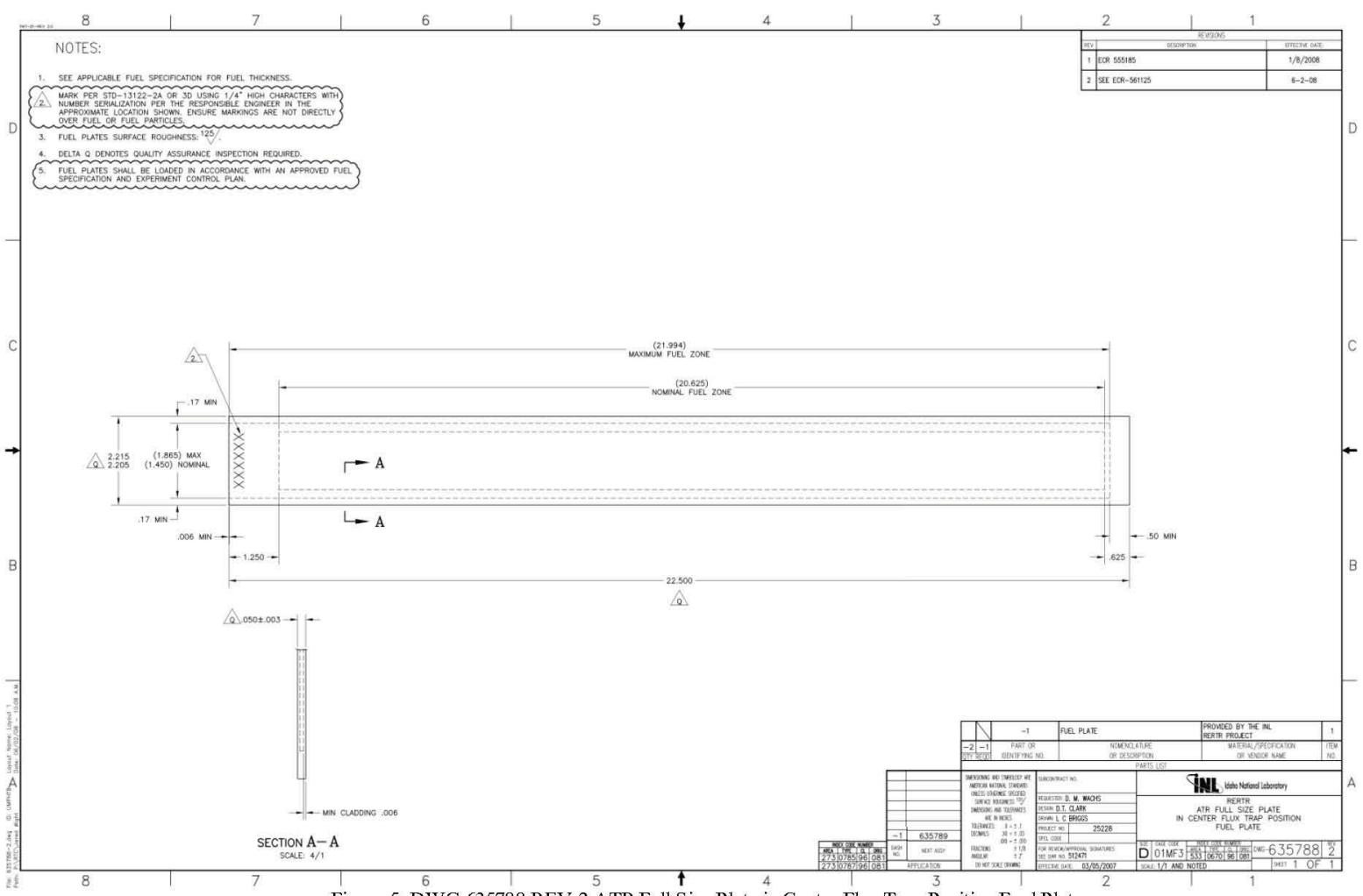

Figure 5. DWG-635788 REV 2 ATR Full Size Plate in Center Flux Trap Position Fuel Plate.

7 


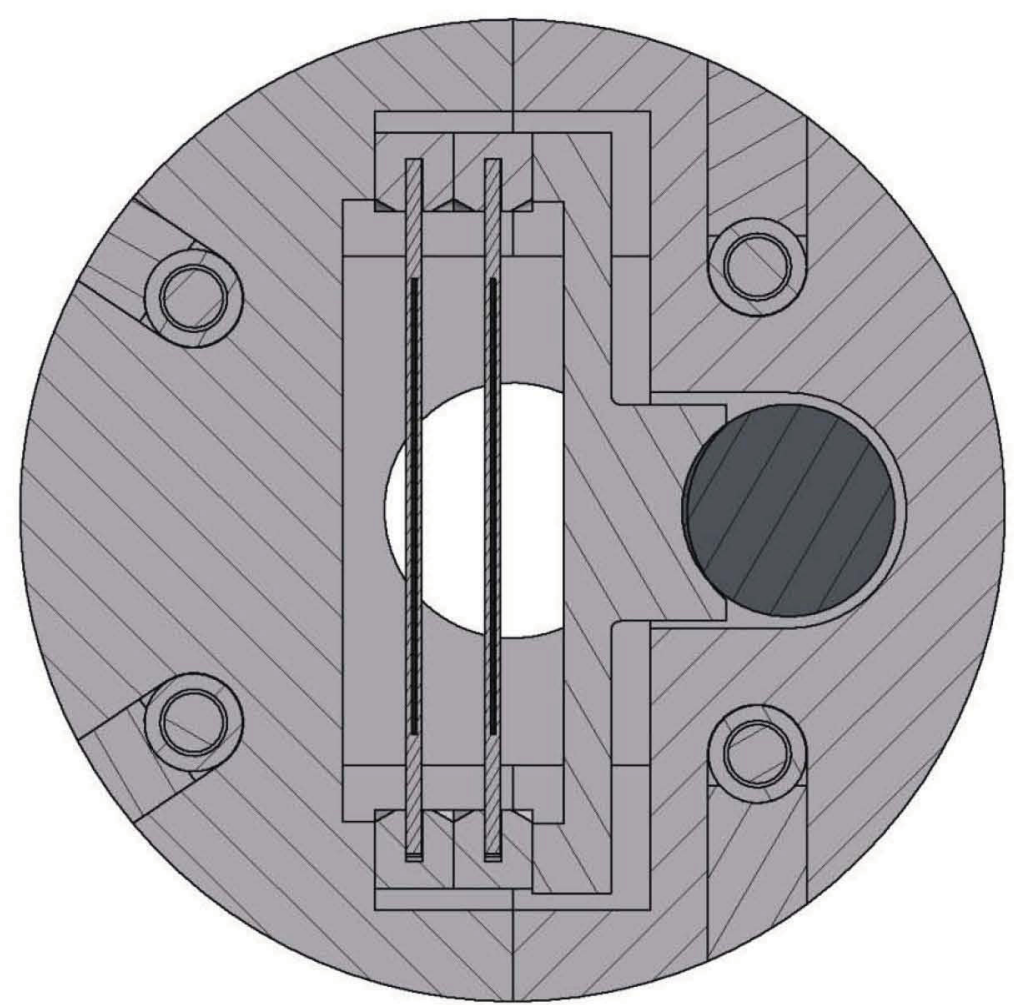

Figure 6. Radial Cross Section of the AFIP Test Train Assembly. 


\section{SAFETY ANALYSIS}

The safety analysis that was performed on the AFIP-2 experiment includes thermal/hydraulic analys is, physics analys is, and structural analysis. The following table (Table 5) summarizes the safety analyses performed on the AFIP-2 experiment.

Table 5. Summary table of the safety analyses done for the AFIP-2 experiment.

\begin{tabular}{|c|l|}
\hline ECAR Number & \multicolumn{1}{|c|}{ Description } \\
\hline ECAR-60 & Physics Analysis of the AFIP Experiments in the Center Flux Trap \\
\hline ECA R-59, Rev. 2 & $\begin{array}{l}\text { Temperature Reactivity Coefficients for the AFIP Experiment in } \\
\text { the Center Flux Trap of ATR }\end{array}$ \\
\hline ECAR-119, Rev. 3 & Thermal Analys is of AFIP-2 Experiment \\
\hline EDF-8209 & AFIP Plate Frame, Test Tra in, and Backup Analys is (Structural) \\
\hline ECAR-121 & Total Flow Evaluations for AFIP and Backups \\
\hline ECAR-126 & RELAP Analysis for AFIP-2 Flow Restrictor \\
\hline
\end{tabular}

\section{IRRADIATION HISTORY}

The AFIP-2 test assembly was irradiated during cycles 141A, 142A and 142B in the ATR Center Flux Trap (CFT). Cycle 141A ran for 32.4 effective full power days (EFPDs) with a center lobe power of 23.4 MW (total core power of 105.4 MW). Cycle 142A ran for 48 EFPDs at a center lobe power of 24.7 MW (total core power of $113.5 \mathrm{MW}$ ). Cycle 142B ran for 52 EFPDs at a center lobe power of $25.4 \mathrm{MW}$ (total core power of $116.8 \mathrm{MW}$ ).

There was one mid-cycle SCRAM during cycle 141A with a duration of 1 day from dates $2 / 7 / 2008$ $2 / 7 / 2008$. There was one mid cycle SCRAM during cycle 142A with a duration of 2 days, from dates $6 / 3 / 2008-6 / 5 / 2008$. There were two mid-cycle SCRAMs during cycle $142 \mathrm{~B}$ with duration of 3 days and 2 days, from dates $7 / 6 / 2008-7 / 9 / 2008$ and $8 / 5 / 2008-8 / 7 / 2008$, respectively. This information is summarized in Table 6.

Table 6. Irradiation history for AFIP-2.

\begin{tabular}{|l|c|c|c|c|c|c|c|c|}
\hline ATR & AFIP \\
CYCLE & Test ID & $\begin{array}{c}\text { AFIP-2 } \\
\text { Frames } \\
\text { Irradiated }\end{array}$ & $\begin{array}{c}\text { Dates } \\
\text { Irrad iated }\end{array}$ & $\begin{array}{c}\text { Cycle } \\
\text { EFPDs }\end{array}$ & $\begin{array}{c}\text { Mid-Cycle } \\
\text { Scram } \\
\text { Decay } \\
\text { Days }\end{array}$ & $\begin{array}{c}\text { Post-Cycle } \\
\text { Decay } \\
\text { Days }\end{array}$ & $\begin{array}{c}\text { Fenter } \\
\text { Trap } \\
\text { Power } \\
\text { (MW) }\end{array}$ & $\begin{array}{c}\text { Total } \\
\text { Core } \\
\text { Power } \\
\text { (MW) }\end{array}$ \\
\hline $141 \mathrm{~A}$ & AFIP-2 & A & $\begin{array}{c}2 / 5 / 2008- \\
3 / 8 / 2008\end{array}$ & 32.4 & 1 & 55 & 23.4 & 105.4 \\
\hline $142 \mathrm{~A}$ & AFIP-2 & A & $\begin{array}{c}5 / 2 / 2008- \\
6 / 21 / 2008\end{array}$ & 48.0 & 2 & 13 & 24.7 & 113.5 \\
\hline 142B & AFIP2/1 & A,B & $\begin{array}{c}7 / 14 / 2008- \\
8 / 30 / 2008\end{array}$ & 52.0 & 5 & 25 & 25.4 & 116.8 \\
\hline
\end{tabular}

The power history for each cycle is obtained as an ATR Surveillance Report from the ATR Data Acquis ition System (DAS). The plots of each lobe power on an hourly basis are shown in Figure 7, Figure 8 and Figure 9 for cycle 141A, 142A and 142B, respectively. 


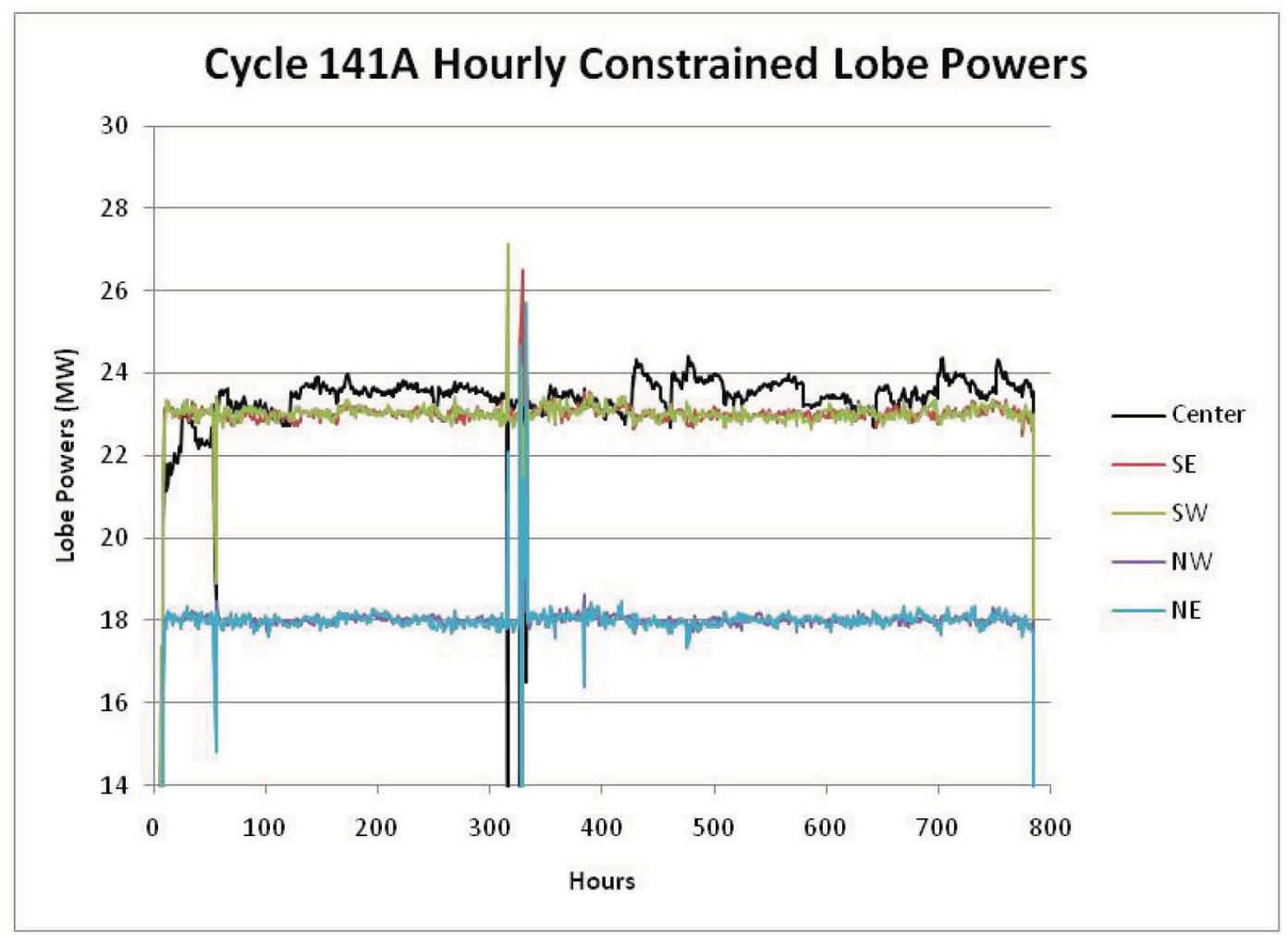

Figure 7. Hourly lobe power history for ATR Cycle 141A.

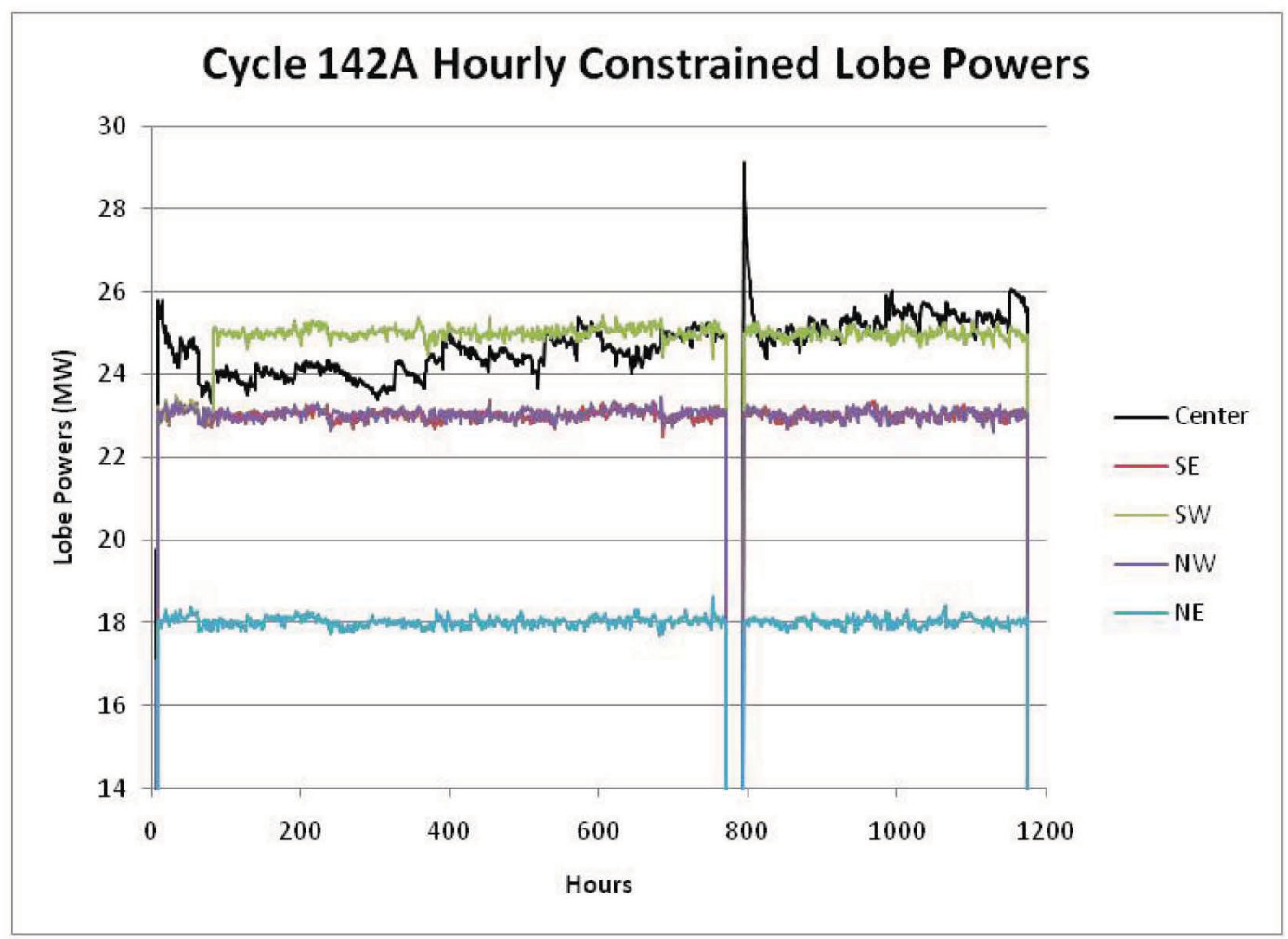

Figure 8. Hourly lobe power history for ATR Cycle 142A. 


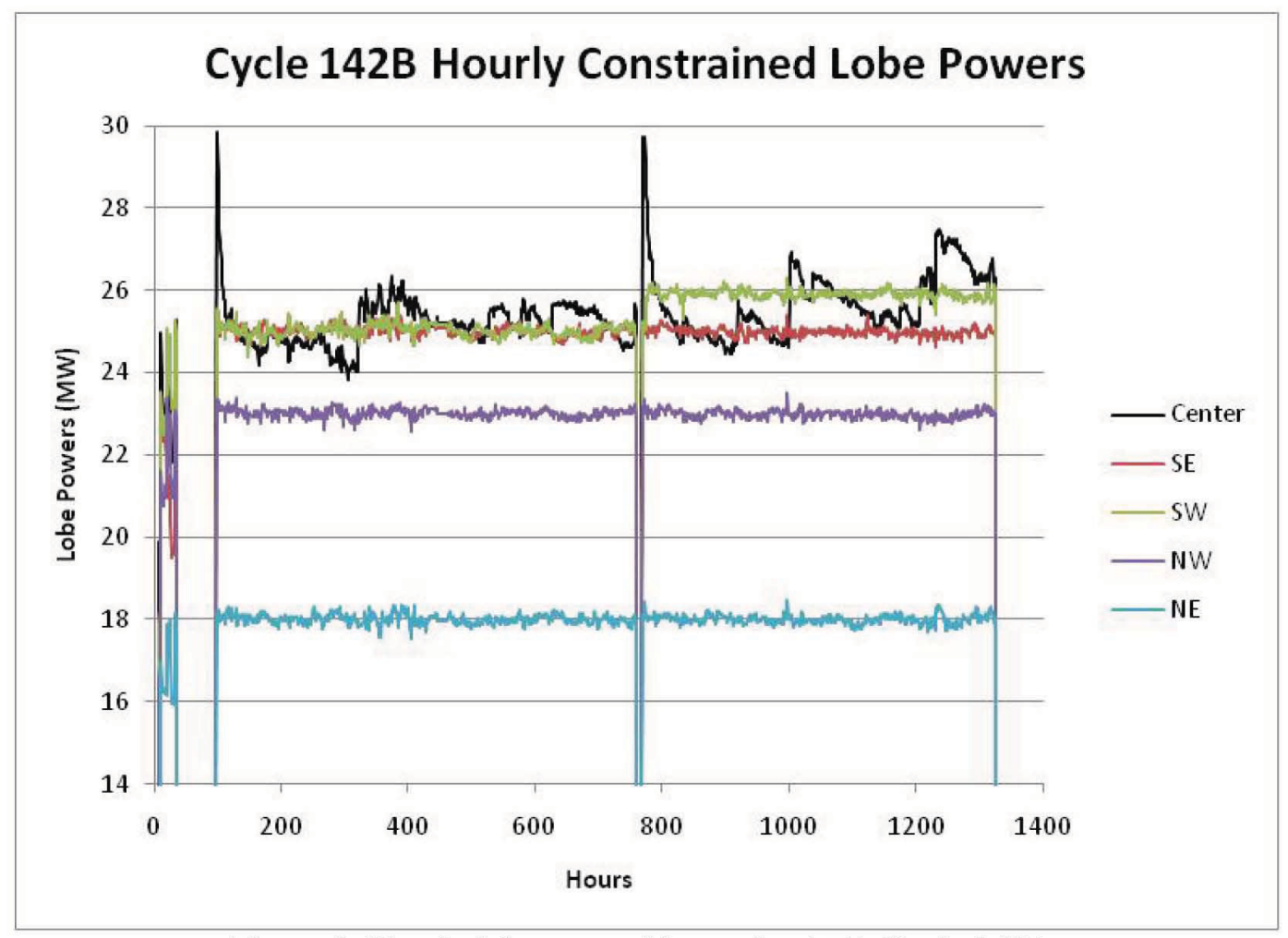

Figure 9. Hourly lobe power history for ATR Cycle 142B. 


\section{AS-RUN NUCLEAR ANALYSIS}

\subsection{Neutronics}

The as-run calculations were performed using the irradiation history in Table 6, the Monte Carlo NParticle (MCNP) code, ORIGEN2.2 and $\mathrm{MCWO}^{3}$. The calculated as-run fission heat rates, fission

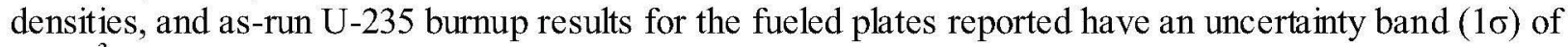
$2.5 \%{ }^{3}$. The time intervals used to calculate the average plate power and burnup is shown in Table 7 .

Table 7. Cycle breakdown.

\begin{tabular}{cccc}
\hline $\begin{array}{c}\text { Time } \\
\text { Interval }\end{array}$ & $\begin{array}{c}141 \mathrm{~A} \\
\text { (days) }\end{array}$ & $\begin{array}{c}142 \mathrm{~A} \\
\text { (days) }\end{array}$ & $\begin{array}{c}142 \mathrm{~B} \\
\text { (days) }\end{array}$ \\
\hline BOC & 0.0 & 0.0 & 0.0 \\
MOC 1 & 15.0 & 16.0 & 16.0 \\
MOC 2 & 10.0 & 16.0 & 13.7 \\
EOC & 7.4 & 16.0 & 22.3 \\
\hline Total EFPDs & 32.4 & 48.0 & 52.0 \\
\hline \hline Cumulative & 32.4 & 80.4 & 132.4 \\
\hline
\end{tabular}

The MCNP-calculated neutronic results reported ${ }^{3}$ were calculated using the fuel foil thickness measurements reported in the as-built data package ${ }^{4}$ as an "in-process" or approximate measurement of the thickness which includes the Zr barrier thickness for plate $2 \mathrm{BZ}$. The actual fuel foil thickness measurements (without the $\mathrm{Zr}$ barrier) were calculated using density measurements and radiography ${ }^{6}$. To correct for the thickness change the fission density (fissions/cc) and the fission power density (W/cc) were scaled to the thickness measurement ratio shown in Table 8.

Table 8. Fuel plate thickness measurements.

\begin{tabular}{|l|c|c|c|}
\hline Plate ID & $\begin{array}{c}\text { "In-Process" } \\
\text { Average Fuel Foil } \\
\text { Thickness (in) }\end{array}$ & $\begin{array}{c}\text { Measured } \\
\text { Average Fuel Foil } \\
\text { Thickness (in) }\end{array}$ & $\begin{array}{c}\text { Thickness } \\
\text { Measurement Ratio }\end{array}$ \\
\hline 2TT & 0.0130 & 0.0140 & 0.929 \\
\hline 2BZ & 0.0157 & 0.0120 & 1.308 \\
\hline
\end{tabular}

The MCNP-calculated end of cycle average plate power and burnup for cycles 141A, 142A and 142B are shown in Table 9. The average plate power and burnup for the time intervals for each cycle are shown in Table 10 through Table 21. The scaled fission power density $(\mathrm{W} / \mathrm{cc})$ and fission dens ity (fissions/cc) are shown in Table 22 through Table 24 . The heat flux $\left(\mathrm{w} / \mathrm{cm}^{2}\right)$ was not scaled since the surface area of the fuel foil (length and width) was represented correctly in the MCNP model. The plots of the power and fission density as a function of the ATR Cycle time interval are in Appendix A. 
Table 9. End of cycle fission power density, surface heat flux, U-235 depletion and fission dens ity for AFIP-2 experiment.

\begin{tabular}{|c|c|c|c|c|c|c|c|c|c|c|c|c|c|}
\hline \multirow[b]{2}{*}{$\begin{array}{c}\text { Plate } \\
\text { DD }\end{array}$} & \multirow[b]{2}{*}{$\begin{array}{l}\text { MCNP Cell } \\
\text { Elevation }^{(a)} \\
\text { (inches) }^{2}\end{array}$} & \multicolumn{4}{|c|}{ EOC 141A } & \multicolumn{4}{|c|}{ EOC 142A } & \multicolumn{4}{|c|}{$\mathrm{EOC} 142 \mathrm{~B}$} \\
\hline & & $\begin{array}{l}\text { Fission } \\
\text { Power } \\
\text { Density } \\
(\mathrm{W} / \mathrm{cc})\end{array}$ & $\begin{array}{c}\text { Surface } \\
\text { Heat Flux } \\
\left(\mathrm{W} / \mathrm{cm}^{2}\right)\end{array}$ & $\begin{array}{c}\% \\
\text { Depletion } \\
\text { U-235 } \\
(\%)\end{array}$ & $\begin{array}{c}\text { Fission } \\
\text { Density } \\
\text { (fission/cc) }\end{array}$ & $\begin{array}{l}\text { Fission } \\
\text { Power } \\
\text { Density } \\
\text { (W/cc) }\end{array}$ & $\begin{array}{c}\text { Surface } \\
\text { Heat Flux } \\
\left(\mathrm{W} / \mathrm{cm}^{2}\right)\end{array}$ & $\begin{array}{c}\% \\
\text { Depletion } \\
\text { U-235 } \\
(\%)\end{array}$ & $\begin{array}{c}\text { Fission } \\
\text { Density } \\
\text { (fission/cc) }\end{array}$ & $\begin{array}{l}\text { Fission } \\
\text { Power } \\
\text { Density } \\
\text { (W/cc) }\end{array}$ & $\begin{array}{c}\text { Surface } \\
\text { Heat Flux } \\
\left(\mathrm{W} / \mathrm{cm}^{2}\right)\end{array}$ & $\begin{array}{c}\% \% \\
\text { Depletion } \\
\text { U-235 } \\
(\%)\end{array}$ & $\begin{array}{c}\text { Fission } \\
\text { Density } \\
\text { (fission/ce) }\end{array}$ \\
\hline \multirow{10}{*}{$2 \mathrm{TT}$} & 20.82 & 8271.31 & 136.56 & $12.36 \%$ & $8.23 \mathrm{E}+20$ & 8299.14 & 137.02 & $29.11 \%$ & $1.98 \mathrm{E}+21$ & 6861.53 & 113.28 & $43.44 \%$ & $3.01 \mathrm{E}+21$ \\
\hline & 18.76 & 10482.66 & 173.07 & $15.86 \%$ & $1.06 \mathrm{E}+21$ & 9975.38 & 164.69 & $36.22 \%$ & $2.50 E+21$ & 7844.20 & 129.51 & $52.70 \%$ & $3.73 \mathrm{E}+21$ \\
\hline & 16.70 & 12522.01 & 206.74 & $18.81 \%$ & $1.27 \mathrm{E}+21$ & 11322.63 & 186.94 & $42.46 \%$ & $2.97 \mathrm{E}+21$ & 8604.32 & 142.06 & $60.51 \%$ & $4.37 \mathrm{E}+21$ \\
\hline & 14.64 & 14075.90 & 232.39 & $21.31 \%$ & $1.45 \mathrm{E}+21$ & 12167.86 & 200.89 & $47.42 \%$ & $3.36 \mathrm{E}+21$ & 8934.20 & 147.50 & $66.14 \%$ & $4.85 \mathrm{E}+21$ \\
\hline & 12.58 & 15494.80 & 255.82 & $23.79 \%$ & $1.63 \mathrm{E}+21$ & 12805.42 & 211.42 & $51.68 \%$ & $3.70 \mathrm{E}+21$ & 9124.25 & 150.64 & $70.73 \%$ & $5.26 \mathrm{E}+21$ \\
\hline & 10.52 & 16376.17 & 270.37 & $25.19 \%$ & $1.74 \mathrm{E}+21$ & 13055.87 & 215.55 & $54.39 \%$ & $3.93 \mathrm{E}+21$ & 9226.58 & 152.33 & $73.71 \%$ & $5.54 \mathrm{E}+21$ \\
\hline & 8.46 & 17233.90 & 284.53 & $26.58 \%$ & $1.84 \mathrm{E}+21$ & 13203.21 & 217.98 & $56.34 \%$ & $4.09 E+21$ & 9137.28 & 150.86 & $75.63 \%$ & $5.73 \mathrm{E}+21$ \\
\hline & 6.40 & 17606.55 & 290.68 & $27.30 \%$ & $1.90 \mathrm{E}+21$ & 13302.62 & 219.63 & $58.14 \%$ & $4.25 \mathrm{E}+21$ & 9126.46 & 150.68 & $77.43 \%$ & $5.90 \mathrm{E}+21$ \\
\hline & 4.34 & 18005.05 & 297.26 & $27.89 \%$ & $1.94 \mathrm{E}+21$ & 13551.17 & 223.73 & $59.13 \%$ & $4.33 \mathrm{E}+21$ & 9073.70 & 149.81 & $78.25 \%$ & $5.98 \mathrm{E}+21$ \\
\hline & 2.28 & 18494.33 & 305.34 & $28.58 \%$ & $2.00 \mathrm{E}+21$ & 13259.86 & 218.92 & $59.92 \%$ & $4.40 \mathrm{E}+21$ & 9044.86 & 149.33 & $79.30 \%$ & $6.09 \mathrm{E}+21$ \\
\hline \multirow{10}{*}{$2 \mathrm{BZ}$} & -2.28 & $1 \overline{14344.12}$ & 286.01 & $\overline{29.76 \%}$ & 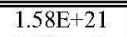 & 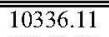 & 206.09 & $61.62 \%$ & $3.45 \mathrm{E}+21$ & 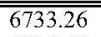 & 134.25 & $80.57 \%$ & $40.72 \mathrm{E}+21$ \\
\hline & -4.34 & 13978.36 & 278.71 & $28.90 \%$ & $1.53 \mathrm{E}+21$ & 10156.39 & 202.51 & $60.36 \%$ & $3.37 \mathrm{E}+21$ & 6916.62 & 137.91 & $79.46 \%$ & $4.65 \mathrm{E}+21$ \\
\hline & -6.40 & 13924.06 & 277.63 & $28.55 \%$ & $1.51 \mathrm{E}+21$ & 10035.89 & 200.11 & $59.60 \%$ & $3.32 \mathrm{E}+21$ & 6974.93 & 139.07 & $78.85 \%$ & $4.59 \mathrm{E}+21$ \\
\hline & -8.46 & 13744.72 & 274.06 & $28.07 \%$ & $1.48 \mathrm{E}+21$ & 10073.53 & 200.86 & $58.81 \%$ & $3.27 \mathrm{E}+21$ & 6974.34 & 139.06 & $78.04 \%$ & $4.54 \mathrm{E}+21$ \\
\hline & -10.52 & 13293.57 & 265.06 & $27.12 \%$ & $1.43 E+21$ & 9906.56 & 197.53 & $57.21 \%$ & $3.16 \mathrm{E}+21$ & 6872.93 & 137.04 & $76.27 \%$ & $4.39 \mathrm{E}+21$ \\
\hline & -12.58 & 12521.51 & 249.67 & $25.52 \%$ & $1.34 \mathrm{E}+21$ & 9715.02 & 193.71 & $54.74 \%$ & $3.00 \mathrm{E}+21$ & 6907.16 & 137.72 & $73.99 \%$ & $4.22 \mathrm{E}+21$ \\
\hline & -14.64 & 11771.63 & 234.71 & $23.81 \%$ & $1.24 \mathrm{E}+21$ & 9557.31 & 190.56 & $51.69 \%$ & $2.81 \mathrm{E}+21$ & 6875.24 & 137.09 & $70.72 \%$ & $3.99 \mathrm{E}+21$ \\
\hline & -16.70 & 10733.32 & 214.01 & $21.48 \%$ & $1.11 \mathrm{E}+21$ & 9027.19 & 179.99 & $47.40 \%$ & $2.55 \mathrm{E}+21$ & 6745.72 & 134.50 & $66.09 \%$ & $3.68 \mathrm{E}+21$ \\
\hline & -18.76 & 9389.84 & 187.22 & $18.67 \%$ & $9.60 \mathrm{E}+20$ & 8402.09 & 167.53 & $42.05 \%$ & $2.23 E+21$ & 6423.32 & 128.07 & $59.90 \%$ & $3.28 \mathrm{E}+21$ \\
\hline & -20.82 & 7726.90 & 154.07 & $15.28 \%$ & $7.80 \mathrm{E}+20$ & 7389.82 & 147.35 & $35.36 \%$ & $1.85 \mathrm{E}+21$ & 5915.91 & 117.96 & $51.92 \%$ & $2.78 \mathrm{E}+21$ \\
\hline MAX & NA & 18494.33 & 305.34 & $29.76 \%$ & $2.00 \mathrm{E}+21$ & 13551.17 & 223.73 & $61.62 \%$ & $\overline{4} 4.40 \mathrm{E}+21$ & 9226.58 & 152.33 & $80.57 \%$ & $\overline{76.09 \mathrm{E}+21}$ \\
\hline MIN & NA & 7726.90 & 136.56 & $12.36 \%$ & $7.80 \mathrm{E}+20$ & 7389.82 & 137.02 & $29.11 \%$ & $1.85 \mathrm{E}+21$ & 5915.91 & 113.28 & $43.44 \%$ & $2.78 \mathrm{E}+21$ \\
\hline AVG & NA & 13499.54 & 243.70 & $23.74 \%$ & $1.43 \mathrm{E}+21$ & 10777.15 & 194.15 & $51.18 \%$ & $3.23 \mathrm{E}+21$ & 7715.84 & 138.93 & $69.68 \%$ & $4.56 \mathrm{E}+21$ \\
\hline
\end{tabular}

(a) Elevations for MCNP cells are reported at cell centroids relative to the modeled core axial midpla ne at 24.0 inches. 
Table 10. Cycle 141A, MCNP-Calculated heat generation rate and neutron flux for AFIP-2 fuel foils 0 EFPD (BOC) Center Lobe Power at 23.4 MW. ${ }^{3}$

\begin{tabular}{|c|c|c|c|c|c|c|}
\hline $\begin{array}{c}\text { Plate } \\
\text { ID }\end{array}$ & Cell & $\begin{array}{c}\text { MCNP Cell } \\
\text { Elevation }^{(a)} \\
\text { (inches) }^{\text {Anch }}\end{array}$ & $\begin{array}{c}\text { Fission } \\
\text { Power Density } \\
(\text { W/cc) }\end{array}$ & $\begin{array}{c}\text { Fission } \\
\text { Heat Rate } \\
(\mathrm{W} / \mathrm{g})\end{array}$ & $\begin{array}{c}\text { Surface } \\
\text { Heat Flux } \\
\left(\mathrm{W} / \mathrm{cm}^{2}\right)\end{array}$ & $\begin{array}{c}\text { Neutron } \\
\text { Flux } \\
\left(\mathrm{n} / \mathrm{cm}^{2} \mathrm{sec}\right)\end{array}$ \\
\hline & A-1 & 20.82 & 8650.68 & 510.17 & 142.82 & $4.54 \mathrm{E}+14$ \\
& A-2 & 18.76 & 11274.41 & 664.91 & 186.14 & $5.93 \mathrm{E}+14$ \\
& A-3 & 16.70 & 13421.49 & 791.53 & 221.59 & $7.18 \mathrm{E}+14$ \\
& A-4 & 14.64 & 15490.94 & 913.58 & 255.76 & $8.27 \mathrm{E}+14$ \\
& A-5 & 12.58 & 17708.26 & 1044.35 & 292.36 & $9.17 \mathrm{E}+14$ \\
& A-6 & 10.52 & 18862.51 & 1112.42 & 311.42 & $9.81 \mathrm{E}+14$ \\
& A-7 & 8.46 & 20009.14 & 1180.04 & 330.35 & $1.02 \mathrm{E}+15$ \\
& A-8 & 6.40 & 20728.52 & 1222.47 & 342.23 & $1.06 \mathrm{E}+15$ \\
& A-9 & 4.34 & 21171.41 & 1248.59 & 349.54 & $1.07 \mathrm{E}+15$ \\
& A-10 & 2.28 & 21752.64 & 1282.86 & 359.14 & $1.09 \mathrm{E}+15$ \\
\hline \hline & A-11 & -2.28 & 17461.74 & 1247.42 & 348.17 & $1.08 \mathrm{E}+15$ \\
& A-12 & -4.34 & 16921.82 & 1208.85 & 337.40 & $1.08 \mathrm{E}+15$ \\
& A-13 & -6.40 & 16446.54 & 1174.90 & 327.93 & $1.06 \mathrm{E}+15$ \\
& A-14 & -8.46 & 16166.49 & 1154.89 & 322.34 & $1.04 \mathrm{E}+15$ \\
& A-15 & -10.52 & 15599.23 & 1114.37 & 311.03 & $1.01 \mathrm{E}+15$ \\
& A-16 & -12.58 & 14519.96 & 1037.27 & 289.51 & $9.60 \mathrm{E}+14$ \\
& A-17 & -14.64 & 13436.02 & 959.83 & 267.90 & $8.96 \mathrm{E}+14$ \\
& A-18 & -16.70 & 11901.37 & 850.20 & 237.30 & $8.02 \mathrm{E}+14$ \\
& A-19 & -18.76 & 10183.67 & 727.49 & 203.05 & $6.84 \mathrm{E}+14$ \\
& A-20 & -20.82 & 8251.69 & 589.48 & 164.53 & $5.50 \mathrm{E}+14$ \\
\hline \hline & MAX & NA & 21752.64 & 1282.86 & 359.14 & $1.09 \mathrm{E}+15$ \\
\hline & MIN & NA & 8251.69 & 510.17 & 142.82 & $4.54 \mathrm{E}+14$ \\
\hline & AVG & NA & 15497.93 & 1001.78 & 280.03 & $8.94 \mathrm{E}+14$ \\
\hline
\end{tabular}

(a) Elevations for MCNP cells are reported at cell centroids relative to the modeled core axial mid plane at 24.0 inches.

Table 11. Cycle 141A, MCNP-Calculated HGR, Neutron Flux, Depletion, and Fission Density for AFIP-2 Fuel Foils, 15.0 EFPD (MOC1), Center Lobe Power @ 23.4 MW (15.0 EFPD Cumulative). ${ }^{3}$

\begin{tabular}{|c|c|c|c|c|c|c|c|c|}
\hline $\begin{array}{c}\text { Plate } \\
\text { ID }\end{array}$ & Cell & $\begin{array}{l}\text { MCNP Cell } \\
\text { Elevation }^{(a)} \\
\text { (inches) }^{\text {(inch }}\end{array}$ & $\begin{array}{c}\text { Fission } \\
\text { Power Density } \\
(\mathrm{W} / \mathrm{cc})\end{array}$ & $\begin{array}{l}\text { Fission } \\
\text { Heat Rate } \\
(W / g)\end{array}$ & $\begin{array}{c}\text { Surface } \\
\text { Heat Flux } \\
\left(\mathrm{W} / \mathrm{cm}^{2}\right)\end{array}$ & $\begin{array}{c}\text { Neutron } \\
\text { Flux } \\
\left(\mathrm{n} / \mathrm{cm}^{2} \mathrm{sec}\right)\end{array}$ & $\begin{array}{c}\% \text { Depletion } \\
\text { U-235 } \\
(\%)\end{array}$ & $\begin{array}{c}\text { Fission } \\
\text { Density } \\
\text { (fissions/cc) }\end{array}$ \\
\hline \multirow{10}{*}{$2 \mathrm{TT}$} & A-1 & 20.82 & 8554.12 & 508.64 & 141.23 & $4.62 \mathrm{E}+14$ & $5.82 \%$ & $3.85 \mathrm{E}+20$ \\
\hline & A-2 & 18.76 & 11026.45 & 657.24 & 182.05 & $6.05 \mathrm{E}+14$ & $7.46 \%$ & $5.02 \mathrm{E}+20$ \\
\hline & A-3 & 16.70 & 13296.62 & 794.12 & 219.53 & $7.48 \mathrm{E}+14$ & $8.86 \%$ & $5.98 \mathrm{E}+20$ \\
\hline & A-4 & 14.64 & 15087.08 & 902.71 & 249.09 & $8.50 \mathrm{E}+14$ & $10.10 \%$ & $6.90 \mathrm{E}+20$ \\
\hline & A-5 & 12.58 & 16703.63 & 1001.42 & 275.78 & $9.38 \mathrm{E}+14$ & $11.51 \%$ & $7.89 \mathrm{E}+20$ \\
\hline & A-6 & 10.52 & 17791.34 & 1067.73 & 293.73 & $9.91 \mathrm{E}+14$ & $12.20 \%$ & $8.40 \mathrm{E}+20$ \\
\hline & A-7 & 8.46 & 18880.73 & 1134.14 & 311.72 & $1.04 \mathrm{E}+15$ & $12.82 \%$ & $8.91 \mathrm{E}+20$ \\
\hline & A- 8 & 6.40 & 19330.54 & 1162.02 & 319.15 & $1.06 \mathrm{E}+15$ & $13.29 \%$ & $9.23 \mathrm{E}+20$ \\
\hline & A-9 & 4.34 & 19838.67 & 1192.97 & 327.54 & $1.08 \mathrm{E}+15$ & $13.52 \%$ & $9.43 \mathrm{E}+20$ \\
\hline & A-10 & 2.28 & 20369.03 & 1225.36 & 336.29 & $1.08 \mathrm{E}+15$ & $13.83 \%$ & $9.69 \mathrm{E}+20$ \\
\hline \multirow{13}{*}{$2 \mathrm{BZ}$} & A-11 & -2.28 & 16022.62 & 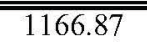 & 319.47 & $=1.08 \mathrm{E}+15$ & $14.61 \%$ & $\overline{77.78 \mathrm{E}+20}$ \\
\hline & A-12 & -4.34 & 15464.81 & 1125.70 & 308.35 & $1.08 \mathrm{E}+15$ & $14.21 \%$ & $7.54 \mathrm{E}+20$ \\
\hline & A-13 & -6.40 & 15477.60 & 1125.98 & 308.61 & $1.07 \mathrm{E}+15$ & $13.85 \%$ & $7.33 \mathrm{E}+20$ \\
\hline & A-14 & -8.46 & 15159.28 & 1102.48 & 302.26 & $1.06 \mathrm{E}+15$ & $13.63 \%$ & $7.20 \mathrm{E}+20$ \\
\hline & A-15 & -10.52 & 14606.63 & 1061.68 & 291.24 & $1.02 \mathrm{E}+15$ & $13.17 \%$ & $6.95 \mathrm{E}+20$ \\
\hline & A-16 & -12.58 & 13700.43 & 994.66 & 273.17 & $9.62 \mathrm{E}+14$ & $12.33 \%$ & $6.47 \mathrm{E}+20$ \\
\hline & A-17 & -14.64 & 12751.70 & 924.69 & 254.26 & $9.02 \mathrm{E}+14$ & $11.46 \%$ & $5.99 \mathrm{E}+20$ \\
\hline & A-18 & -16.70 & 11528.00 & 834.58 & 229.86 & $8.18 \mathrm{E}+14$ & $10.22 \%$ & $5.30 \mathrm{E}+20$ \\
\hline & A-19 & -18.76 & 9962.31 & 719.94 & 198.64 & $6.92 \mathrm{E}+14$ & $8.82 \%$ & $4.54 \mathrm{E}+20$ \\
\hline & A-20 & -20.82 & 8085.92 & 583.05 & 161.23 & $5.63 \mathrm{E}+14$ & $7.20 \%$ & $3.68 \mathrm{E}+20$ \\
\hline & MAX & NA & 20369.03 & 1225.36 & 336.29 & $1.08 \mathrm{E}+15$ & $14.61 \%$ & $9.69 \mathrm{E}+20$ \\
\hline & MIN & $\mathrm{NA}$ & 8085.92 & 508.64 & 141.23 & $4.62 \mathrm{E}+14$ & $5.82 \%$ & $3.68 \mathrm{E}+20$ \\
\hline & $\overline{A V G}$ & NA & 14681.88 & 964.30 & 265.16 & $9.05 \mathrm{E}+14$ & $11.45 \%$ & $6.90 \mathrm{E}+20$ \\
\hline
\end{tabular}

(a) Elevations for MCNP cells a re reported at cell centroids relative to the modeled core axial midplane at 24.0 inches. 
Table 12. Cycle 141A, MCNP-Calculated HGR, Neutron Flux, Depletion, and Fiss ion Density for AFIP-2 Fuel Foils, 25.0 EFPD (MOC2), Center Lobe Power@23.4 MW (25.0 EFPD Cumulative). ${ }^{3}$

\begin{tabular}{|c|c|c|c|c|c|c|c|c|}
\hline $\begin{array}{c}\text { Plate } \\
\text { ID }\end{array}$ & Cell & $\begin{array}{l}\text { MCNP Cell } \\
\text { Elevation }^{(a)} \\
\text { (inches) }^{\text {(inch }}\end{array}$ & $\begin{array}{c}\text { Fission } \\
\text { Power Density } \\
\text { (W/cc) }\end{array}$ & $\begin{array}{c}\text { Fission } \\
\text { Heat Rate } \\
(\mathrm{W} / \mathrm{g})\end{array}$ & $\begin{array}{c}\text { Surface } \\
\text { Heat Flux } \\
\left(\mathrm{W} / \mathrm{cm}^{2}\right)\end{array}$ & $\begin{array}{c}\text { Neutron } \\
\text { Flux } \\
\left(\mathrm{n} / \mathrm{cm}^{2} \mathrm{sec}\right)\end{array}$ & $\begin{array}{c}\% \text { Depletion } \\
\mathrm{U}-235 \\
(\%)\end{array}$ & $\begin{array}{c}\text { Fission } \\
\text { Density } \\
\text { (fissions/cc) }\end{array}$ \\
\hline \multirow{10}{*}{$2 \mathrm{TT}$} & $\mathrm{A}-1$ & 20.82 & 8399.21 & 502.14 & 138.67 & $4.61 \mathrm{E}+14$ & $9.63 \%$ & $6.39 \mathrm{E}+20$ \\
\hline & A-2 & 18.76 & 10709.90 & 642.74 & 176.82 & $6.04 \mathrm{E}+14$ & $12.36 \%$ & $8.29 \mathrm{E}+20$ \\
\hline & A-3 & 16.70 & 12851.64 & 773.84 & 212.18 & $7.47 \mathrm{E}+14$ & $14.62 \%$ & $9.92 \mathrm{E}+20$ \\
\hline & A-4 & 14.64 & 14493.54 & 875.29 & 239.29 & $8.48 \mathrm{E}+14$ & $16.63 \%$ & $1.14 \mathrm{E}+21$ \\
\hline & A-5 & 12.58 & 16013.19 & 970.06 & 264.38 & $9.36 \mathrm{E}+14$ & $18.74 \%$ & $1.28 \mathrm{E}+21$ \\
\hline & A-6 & 10.52 & 17006.26 & 1031.93 & 280.77 & $9.91 \mathrm{E}+14$ & $19.82 \%$ & $1.37 \mathrm{E}+21$ \\
\hline & A-7 & 8.46 & 17922.78 & 1089.21 & 295.91 & $1.04 \mathrm{E}+15$ & $20.91 \%$ & $1.45 \mathrm{E}+21$ \\
\hline & A-8 & 6.40 & 18327.58 & 1114.90 & 302.59 & $1.06 \mathrm{E}+15$ & $21.54 \%$ & $1.50 \mathrm{E}+21$ \\
\hline & A-9 & 4.34 & 18795.26 & 1144.13 & 310.31 & $1.08 \mathrm{E}+15$ & $22.00 \%$ & $1.53 \mathrm{E}+21$ \\
\hline & A- 10 & 2.28 & 19319.77 & 1177.08 & 318.97 & $1.08 \mathrm{E}+15$ & $22.53 \%$ & $1.57 \mathrm{E}+21$ \\
\hline \multirow{13}{*}{$2 \mathrm{BZ}$} & A-11 & -2.28 & 15044.85 & 1108.85 & 299.98 & $1.08 \mathrm{E}+15$ & $23.57 \%$ & $1.25 \mathrm{E}+21$ \\
\hline & A-12 & -4.34 & 14624.88 & 1077.00 & 291.61 & $1.08 \mathrm{E}+15$ & $22.88 \%$ & $1.21 \mathrm{E}+21$ \\
\hline & A-13 & -6.40 & 14583.07 & 1073.38 & 290.77 & $1.07 \mathrm{E}+15$ & $22.53 \%$ & $1.19 \mathrm{E}+21$ \\
\hline & A-14 & -8.46 & 14389.00 & 1058.45 & 286.90 & $1.05 \mathrm{E}+15$ & $22.13 \%$ & $1.17 \mathrm{E}+21$ \\
\hline & A-15 & -10.52 & 13861.76 & 1018.62 & 276.39 & $1.02 \mathrm{E}+15$ & $21.39 \%$ & $1.13 \mathrm{E}+21$ \\
\hline & A-16 & -12.58 & 13041.83 & 956.69 & 260.04 & $9.61 \mathrm{E}+14$ & $20.10 \%$ & $1.05 \mathrm{E}+21$ \\
\hline & A-17 & -14.64 & 12193.19 & 892.70 & 243.12 & $9.01 \mathrm{E}+14$ & $18.70 \%$ & $9.77 \mathrm{E}+20$ \\
\hline & A- 18 & -16.70 & 11061.23 & 807.83 & 220.55 & $8.17 \mathrm{E}+14$ & $16.83 \%$ & $8.72 \mathrm{E}+20$ \\
\hline & A- 19 & -18.76 & 9636.46 & 701.67 & 192.14 & $6.92 \mathrm{E}+14$ & $14.58 \%$ & $7.49 \mathrm{E}+20$ \\
\hline & A-20 & -20.82 & 7873.45 & 571.24 & 156.99 & $5.64 \mathrm{E}+14$ & $11.91 \%$ & $6.07 \mathrm{E}+20$ \\
\hline & MAX & $\mathrm{NA}$ & 19319.77 & 1177.08 & 318.97 & $1.08 \mathrm{E}+15$ & $23.57 \%$ & $1.57 \mathrm{E}+21$ \\
\hline & MIN & $\overline{\mathrm{NA}}$ & 7873.45 & 502.14 & 138.67 & $4.61 \mathrm{E}+14$ & $9.63 \%$ & $6.07 \mathrm{E}+20$ \\
\hline & $\overline{A V G}$ & NA & 14007.44 & 929.39 & 252.92 & $9.04 \mathrm{E}+14$ & $18.67 \%$ & $1.13 \mathrm{E}+21$ \\
\hline
\end{tabular}

(a) Elevations for MCNP cells are reported at cell centroids relative to the modeled core axial midplane at 24.0 inches.

Table 13. Cycle 141A, MCNP-Calculated HGR, Neutron Flux, Depletion, and Fiss ion Density for AFIP-2 Fuel Foils, 32.4 EFPD (EOC), Center Lobe Power @ 23.4 MW (32.4 EFPD Cumulative). ${ }^{3}$

\begin{tabular}{|c|c|c|c|c|c|c|c|c|}
\hline $\begin{array}{c}\text { Plate } \\
\text { ID }\end{array}$ & Cell & $\begin{array}{l}\text { MCNP Cell } \\
\text { Elevation }^{(a)} \\
\text { (inches) }^{\text {(inces }}\end{array}$ & $\begin{array}{c}\text { Fission } \\
\text { Power Density } \\
(\mathrm{W} / \mathrm{cc})\end{array}$ & $\begin{array}{c}\text { Fission } \\
\text { Heat Rate } \\
(\mathrm{W} / \mathrm{g})\end{array}$ & $\begin{array}{c}\text { Surface } \\
\text { Heat Flux } \\
\left(\mathrm{W} / \mathrm{cm}^{2}\right)\end{array}$ & $\begin{array}{c}\text { Neutron } \\
\text { Flux } \\
\left(\mathrm{n} / \mathrm{cm}^{2} \mathrm{sec}\right) \\
\end{array}$ & $\begin{array}{c}\% \text { Depletion } \\
\text { U-235 } \\
(\%)\end{array}$ & $\begin{array}{c}\text { Fission } \\
\text { Density } \\
\text { (fissions/cc) }\end{array}$ \\
\hline \multirow{10}{*}{$2 \mathrm{TT}$} & A-1 & 20.82 & 8271.31 & 496.37 & 136.56 & $4.61 \mathrm{E}+14$ & $12.36 \%$ & $8.23 \mathrm{E}+20$ \\
\hline & A-2 & 18.76 & 10482.66 & 632.27 & 173.07 & $6.03 \mathrm{E}+14$ & $15.86 \%$ & $1.06 \mathrm{E}+21$ \\
\hline & A-3 & 16.70 & 12522.01 & 758.58 & 206.74 & $7.46 \mathrm{E}+14$ & $18.81 \%$ & $1.27 \mathrm{E}+21$ \\
\hline & A-4 & 14.64 & 14075.90 & 855.88 & 232.39 & $8.45 \mathrm{E}+14$ & $21.31 \%$ & $1.45 \mathrm{E}+21$ \\
\hline & A-5 & 12.58 & 15494.80 & 945.71 & 255.82 & $9.35 \mathrm{E}+14$ & $23.79 \%$ & $1.63 \mathrm{E}+21$ \\
\hline & A- 6 & 10.52 & 16376.17 & 1001.61 & 270.37 & $9.90 \mathrm{E}+14$ & $25.19 \%$ & $1.74 \mathrm{E}+21$ \\
\hline & A-7 & 8.46 & 17233.90 & 1056.29 & 284.53 & $1.04 \mathrm{E}+15$ & $26.58 \%$ & $1.84 \mathrm{E}+21$ \\
\hline & A- 8 & 6.40 & 17606.55 & 1080.31 & 290.68 & $1.06 \mathrm{E}+15$ & $27.30 \%$ & $1.90 \mathrm{E}+21$ \\
\hline & A-9 & 4.34 & 18005.05 & 1105.78 & 297.26 & $1.08 \mathrm{E}+15$ & $27.89 \%$ & $1.94 \mathrm{E}+21$ \\
\hline & A- 10 & 2.28 & 18494.33 & 1137.06 & 305.34 & $1.08 \mathrm{E}+15$ & $28.58 \%$ & $2.00 \mathrm{E}+21$ \\
\hline \multirow{13}{*}{$2 \mathrm{BZ}$} & A-11 & -2.28 & 14344.12 & 1066.34 & 286.01 & $1.08 \mathrm{E}+15$ & $29.76 \%$ & $1.58 \mathrm{E}+21$ \\
\hline & A-12 & -4.34 & 13978.36 & 1037.88 & 278.71 & $1.08 \mathrm{E}+15$ & $28.90 \%$ & $1.53 \mathrm{E}+21$ \\
\hline & A-13 & -6.40 & 13924.06 & 1033.33 & 277.63 & $1.07 \mathrm{E}+15$ & $28.55 \%$ & $1.51 \mathrm{E}+21$ \\
\hline & A-14 & -8.46 & 13744.72 & 1019.45 & 274.06 & $1.05 \mathrm{E}+15$ & $28.07 \%$ & $1.48 \mathrm{E}+21$ \\
\hline & A-15 & -10.52 & 13293.57 & 984.58 & 265.06 & $1.02 \mathrm{E}+15$ & $27.12 \%$ & $1.43 \mathrm{E}+21$ \\
\hline & A-16 & -12.58 & 12521.51 & 925.38 & 249.67 & $9.58 \mathrm{E}+14$ & $25.52 \%$ & $1.34 \mathrm{E}+21$ \\
\hline & A-17 & -14.64 & 11771.63 & 867.79 & 234.71 & $9.01 \mathrm{E}+14$ & $23.81 \%$ & $1.24 \mathrm{E}+21$ \\
\hline & A-18 & -16.70 & 10733.32 & 788.87 & 214.01 & $8.18 \mathrm{E}+14$ & $21.48 \%$ & $1.11 \mathrm{E}+21$ \\
\hline & A-19 & -18.76 & 9389.84 & 687.41 & 187.22 & $6.92 \mathrm{E}+14$ & $18.67 \%$ & $9.60 \mathrm{E}+20$ \\
\hline & A-20 & -20.82 & 7726.90 & 563.15 & 154.07 & $5.63 \mathrm{E}+14$ & $15.28 \%$ & $7.80 \mathrm{E}+20$ \\
\hline & MAX & $\mathrm{NA}$ & 18494.33 & 1137.06 & 305.34 & $1.08 \mathrm{E}+15$ & $29.76 \%$ & $2.00 \mathrm{E}+21$ \\
\hline & MIN & $\mathrm{NA}$ & 7726.90 & 496.37 & 136.56 & $4.61 \mathrm{E}+14$ & $12.36 \%$ & $7.80 \mathrm{E}+20$ \\
\hline & $\overline{A V G}$ & $\mathrm{NA}$ & 13499.54 & 902.20 & 243.70 & $9.03 \mathrm{E}+14$ & $23.74 \%$ & $1.43 \mathrm{E}+21$ \\
\hline
\end{tabular}

(a) Elevations for MCNP cells a re reported at cell centroids relative to the modeled core axial midplane at 24.0 inches. 
Table 14. Cycle 142A, MCNP-Calculated HGR and Neutron Flux for AFIP-2 Fuel Foils, 0 EFPD (BOC), Center Lobe Power@24.7 MW.

\begin{tabular}{|c|c|c|c|c|c|c|}
\hline $\begin{array}{c}\text { Plate } \\
\text { ID }\end{array}$ & Cell & $\begin{array}{c}\text { MCNP Cell } \\
\text { Elevation }^{\text {(a) }} \\
\text { (inches) }\end{array}$ & $\begin{array}{c}\text { Fission } \\
\text { Power Density } \\
\text { (W/cc) }\end{array}$ & $\begin{array}{c}\text { Fission } \\
\text { Heat Rate } \\
\text { (W/g) }\end{array}$ & $\begin{array}{c}\text { Surface } \\
\text { Heat Flux } \\
\left.\text { (W/cm }^{2}\right)\end{array}$ & $\begin{array}{c}\text { Neutron } \\
\text { Flux } \\
\left(\mathrm{n} / \mathrm{cm}^{2} \text { sec) }\right.\end{array}$ \\
\hline & A-1 & 20.82 & 7615.61 & 457.03 & 125.73 & $4.39 \mathrm{E}+14$ \\
& A-2 & 18.76 & 9571.95 & 577.42 & 158.03 & $5.79 \mathrm{E}+14$ \\
& A-3 & 16.70 & 11469.26 & 694.90 & 189.36 & $7.15 \mathrm{E}+14$ \\
& A-4 & 14.64 & 13162.43 & 800.33 & 217.31 & $8.27 \mathrm{E}+14$ \\
2TT & A-5 & 12.58 & 14321.50 & 874.06 & 236.45 & $9.21 \mathrm{E}+14$ \\
& A-6 & 10.52 & 15497.25 & 947.82 & 255.86 & $9.87 \mathrm{E}+14$ \\
& A-7 & 8.46 & 16210.20 & 993.52 & 267.63 & $1.03 \mathrm{E}+15$ \\
& A-8 & 6.40 & 17008.99 & 1043.61 & 280.82 & $1.07 \mathrm{E}+15$ \\
& A-9 & 4.34 & 17281.40 & 1061.30 & 285.32 & $1.09 \mathrm{E}+15$ \\
& A-10 & 2.28 & 17679.46 & 1086.93 & 291.89 & $1.10 \mathrm{E}+15$ \\
\hline \hline & A-11 & -2.28 & 13738.58 & 1021.28 & 273.93 & $1.10 \mathrm{E}+15$ \\
& A-12 & -4.34 & 13517.28 & 1003.61 & 269.52 & $1.11 \mathrm{E}+15$ \\
& A-13 & -6.40 & 13287.57 & 986.08 & 264.94 & $1.09 \mathrm{E}+15$ \\
& A-14 & -8.46 & 13090.26 & 970.90 & 261.01 & $1.07 \mathrm{E}+15$ \\
2BZ & A-15 & -10.52 & 12577.18 & 931.49 & 250.78 & $1.03 \mathrm{E}+15$ \\
& A-16 & -12.58 & 11991.25 & 886.18 & 239.09 & $9.76 \mathrm{E}+14$ \\
& A-17 & -14.64 & 11171.02 & 823.65 & 222.74 & $9.02 \mathrm{E}+14$ \\
& A-18 & -16.70 & 10095.81 & 742.00 & 201.30 & $8.11 \mathrm{E}+14$ \\
& A-19 & -18.76 & 8784.70 & 643.10 & 175.16 & $6.85 \mathrm{E}+14$ \\
& A-20 & -20.82 & 7268.14 & 529.71 & 144.92 & $5.45 \mathrm{E}+14$ \\
\hline \hline & MAX & NA & 17679.46 & 1086.93 & 291.89 & $1.11 \mathrm{E}+15$ \\
\hline & MIN & NA & 7268.14 & 457.03 & 125.73 & $4.39 \mathrm{E}+14$ \\
\hline & AVG & NA & 12766.99 & 853.74 & 230.59 & $9.04 \mathrm{E}+14$ \\
\hline
\end{tabular}

(a) Elevations for MCNP cells are reported at cell centroids relative to the modeled core axial midplane at 24.0 inches.

Table 15. Cycle 142A, MCNP-Calculated HGR, Neutron Flux, Depletion, and Fiss ion Density for AFIP-2 Fuel Foils, 16.0 EFPD (MOC1), Center Lobe Power@ 24.7 MW (48.4 EFPD Cumulative). ${ }^{3}$

\begin{tabular}{|c|c|c|c|c|c|c|c|c|}
\hline $\begin{array}{c}\text { Plate } \\
\text { ID }\end{array}$ & Cell & $\begin{array}{l}\text { MCNP Cell } \\
\text { Elevation }^{(a)} \\
\text { (inches) }^{\text {(inche }}\end{array}$ & $\begin{array}{c}\text { Fission } \\
\text { Power Density } \\
\text { (W/cc) }\end{array}$ & $\begin{array}{c}\text { Fission } \\
\text { Heat Rate } \\
(\mathrm{W} / \mathrm{g})\end{array}$ & $\begin{array}{c}\text { Surface } \\
\text { Heat Flux } \\
\left(\mathrm{W} / \mathrm{cm}^{2}\right)\end{array}$ & $\begin{array}{c}\text { Neutron } \\
\text { Flux } \\
\left(\mathrm{n} / \mathrm{cm}^{2} \mathrm{sec}\right) \\
\end{array}$ & $\begin{array}{c}\% \text { Depletion } \\
\text { U-235 } \\
(\%)\end{array}$ & $\begin{array}{c}\text { Fission } \\
\text { Density } \\
\text { (fissions/cc) }\end{array}$ \\
\hline \multirow{10}{*}{$2 \mathrm{TT}$} & $\mathrm{A}-1$ & 20.82 & 8342.38 & 504.44 & 137.73 & $4.82 \mathrm{E}+14$ & $17.56 \%$ & $1.18 \mathrm{E}+21$ \\
\hline & A-2 & 18.76 & 10301.69 & 627.35 & 170.08 & $6.30 \mathrm{E}+14$ & $22.34 \%$ & $1.51 \mathrm{E}+21$ \\
\hline & A-3 & 16.70 & 12153.75 & 744.79 & 200.66 & $7.72 \mathrm{E}+14$ & $26.47 \%$ & $1.81 \mathrm{E}+21$ \\
\hline & A-4 & 14.64 & 13568.46 & 836.03 & 224.02 & $8.76 \mathrm{E}+14$ & $30.00 \%$ & $2.07 \mathrm{E}+21$ \\
\hline & A-5 & 12.58 & 14727.72 & 911.74 & 243.15 & $9.71 \mathrm{E}+14$ & $33.16 \%$ & $2.31 \mathrm{E}+21$ \\
\hline & A-6 & 10.52 & 15407.44 & 957.04 & 254.38 & $1.03 \mathrm{E}+15$ & $35.24 \%$ & $2.47 \mathrm{E}+21$ \\
\hline & A-7 & 8.46 & 15896.68 & 990.19 & 262.45 & $1.07 \mathrm{E}+15$ & $36.98 \%$ & $2.60 \mathrm{E}+21$ \\
\hline & A- 8 & 6.40 & 16512.70 & 1030.55 & 272.62 & $1.10 \mathrm{E}+15$ & $38.17 \%$ & $2.70 \mathrm{E}+21$ \\
\hline & A-9 & 4.34 & 16838.93 & 1052.12 & 278.01 & $1.12 \mathrm{E}+15$ & $38.93 \%$ & $2.75 \mathrm{E}+21$ \\
\hline & A-10 & 2.28 & 16905.73 & 1057.76 & 279.11 & $1.13 \mathrm{E}+15$ & $39.81 \%$ & $2.82 \mathrm{E}+21$ \\
\hline \multirow{13}{*}{$2 \mathrm{BZ}$} & A-11 & -2.28 & 13237.11 & $\overline{1000.42}$ & 263.93 & $\overline{1.13 \mathrm{E}+15}$ & 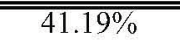 & $2.23 \mathrm{E}+21$ \\
\hline & A-12 & -4.34 & 12986.98 & 980.10 & 258.95 & $1.13 \mathrm{E}+15$ & $40.18 \%$ & $2.17 \mathrm{E}+21$ \\
\hline & A-13 & -6.40 & 12740.93 & 960.66 & 254.04 & $1.11 \mathrm{E}+15$ & $39.70 \%$ & $2.13 \mathrm{E}+21$ \\
\hline & A-14 & -8.46 & 12624.60 & 951.15 & 251.72 & $1.09 \mathrm{E}+15$ & $39.06 \%$ & $2.10 \mathrm{E}+21$ \\
\hline & A-15 & -10.52 & 12215.94 & 918.57 & 243.57 & $1.05 \mathrm{E}+15$ & $37.74 \%$ & $2.02 \mathrm{E}+21$ \\
\hline & A-16 & -12.58 & 11781.86 & 883.24 & 234.92 & $9.98 \mathrm{E}+14$ & $35.73 \%$ & $1.90 \mathrm{E}+21$ \\
\hline & A-17 & -14.64 & 11091.39 & 828.74 & 221.15 & $9.34 \mathrm{E}+14$ & $33.44 \%$ & $1.77 \mathrm{E}+21$ \\
\hline & A-18 & -16.70 & 10048.72 & 747.60 & 200.36 & $8.35 \mathrm{E}+14$ & $30.27 \%$ & $1.59 \mathrm{E}+21$ \\
\hline & A-19 & -18.76 & 8874.52 & 656.55 & 176.95 & $7.17 \mathrm{E}+14$ & $26.42 \%$ & $1.37 \mathrm{E}+21$ \\
\hline & A-20 & -20.82 & 7522.33 & 552.99 & 149.99 & $5.83 \mathrm{E}+14$ & $21.77 \%$ & $1.12 \mathrm{E}+21$ \\
\hline & MAX & NA & 1016905.73 & $\overline{1057.76}$ & 279.11 & $\bar{~} 1.13 \mathrm{E}+15$ & $4 \quad 41.19 \%$ & $2.82 \mathrm{E}+21$ \\
\hline & MIN & NA & 7522.33 & 504.44 & 137.73 & $4.82 \mathrm{E}+14$ & $17.56 \%$ & $1.12 \mathrm{E}+21$ \\
\hline & $\overline{A V G}$ & $\mathrm{NA}$ & 12688.99 & 859.60 & 228.89 & $9.38 \mathrm{E}+14$ & $33.21 \%$ & $2.03 \mathrm{E}+21$ \\
\hline
\end{tabular}

(a) Elevations for MCNP cells a re reported at cell centroids relative to the modeled core axial midplane at 24.0 inches. 
Table 16. Cycle 142A, MCNP-Calculated HGR, Neutron Flux, Depletion, and Fiss ion Density for AFIP-2 Fuel Foils, 32.0 EFPD (MOC2), Center Lobe Power@ 24.7 MW (64.4 EFPD Cumulative). ${ }^{3}$

\begin{tabular}{|c|c|c|c|c|c|c|c|c|}
\hline $\begin{array}{c}\text { Plate } \\
\text { ID }\end{array}$ & Cell & $\begin{array}{l}\text { MCNP Cell } \\
\text { Elevation }^{(a)} \\
\text { (inches) }^{\text {(inches }}\end{array}$ & $\begin{array}{c}\text { Fission } \\
\text { Power Density } \\
(\mathrm{W} / \mathrm{cc})\end{array}$ & $\begin{array}{c}\text { Fission } \\
\text { Heat Rate } \\
(\mathrm{W} / \mathrm{g})\end{array}$ & $\begin{array}{c}\text { Surface } \\
\text { Heat Flux } \\
\left(\mathrm{W} / \mathrm{cm}^{2}\right)\end{array}$ & $\begin{array}{c}\text { Neutron } \\
\text { Flux } \\
\left(\mathrm{n} / \mathrm{cm}^{2} \mathrm{sec}\right)\end{array}$ & $\begin{array}{c}\% \text { Depletion } \\
\text { U-235 } \\
(\%)\end{array}$ & $\begin{array}{c}\text { Fission } \\
\text { Density } \\
\text { (fissions/cc) }\end{array}$ \\
\hline \multirow{10}{*}{$2 \mathrm{TT}$} & A-1 & 20.82 & 8485.38 & 517.52 & 140.09 & $5.01 \mathrm{E}+14$ & $23.32 \%$ & $1.58 \mathrm{E}+21$ \\
\hline & A-2 & 18.76 & 10455.90 & 643.46 & 172.63 & $6.51 \mathrm{E}+14$ & $29.27 \%$ & $2.00 \mathrm{E}+21$ \\
\hline & A-3 & 16.70 & 12269.34 & 761.22 & 202.57 & $7.98 \mathrm{E}+14$ & $34.50 \%$ & $2.39 \mathrm{E}+21$ \\
\hline & A-4 & 14.64 & 13577.00 & 848.10 & 224.16 & $9.13 \mathrm{E}+14$ & $38.80 \%$ & $2.72 \mathrm{E}+21$ \\
\hline & A-5 & 12.58 & 14617.73 & 918.79 & 241.34 & $1.00 \mathrm{E}+15$ & $42.59 \%$ & $3.00 \mathrm{E}+21$ \\
\hline & A-6 & 10.52 & 15319.63 & 966.59 & 252.93 & $1.06 \mathrm{E}+15$ & $44.99 \%$ & $3.20 \mathrm{E}+21$ \\
\hline & A-7 & 8.46 & 15440.82 & 977.56 & 254.93 & $1.10 \mathrm{E}+15$ & $46.97 \%$ & $3.36 \mathrm{E}+21$ \\
\hline & A-8 & 6.40 & 16097.98 & 1021.63 & 265.78 & $1.14 \mathrm{E}+15$ & $48.45 \%$ & $3.48 \mathrm{E}+21$ \\
\hline & A-9 & 4.34 & 16297.21 & 1035.76 & 269.07 & $1.16 \mathrm{E}+15$ & $49.38 \%$ & $3.55 \mathrm{E}+21$ \\
\hline & A-10 & 2.28 & 16204.14 & 1031.49 & 267.53 & $1.15 \mathrm{E}+15$ & $50.26 \%$ & $3.63 \mathrm{E}+21$ \\
\hline \multirow{13}{*}{$2 \mathrm{BZ}$} & $\overline{\mathrm{A}-11}$ & -2.28 & 1012553.82 & 9964.13 & 250.31 & $1.16 \mathrm{E}+15$ & $\overline{\overline{51.87 \%}}$ & $\overline{2} 2.85 \mathrm{E}+21$ \\
\hline & A-12 & -4.34 & 12341.70 & 946.32 & 246.08 & $1.15 \mathrm{E}+15$ & $50.71 \%$ & $2.78 \mathrm{E}+21$ \\
\hline & A-13 & -6.40 & 12110.50 & 927.57 & 241.47 & $1.13 \mathrm{E}+15$ & $50.08 \%$ & $2.74 \mathrm{E}+21$ \\
\hline & A-14 & -8.46 & 12005.11 & 918.51 & 239.37 & $1.11 \mathrm{E}+15$ & $49.34 \%$ & $2.70 \mathrm{E}+21$ \\
\hline & A-15 & -10.52 & 11750.89 & 896.88 & 234.30 & $1.08 \mathrm{E}+15$ & $47.83 \%$ & $2.60 \mathrm{E}+21$ \\
\hline & A-16 & -12.58 & 11388.48 & 866.21 & 227.07 & $1.03 \mathrm{E}+15$ & $45.53 \%$ & $2.46 \mathrm{E}+21$ \\
\hline & A-17 & -14.64 & 10875.75 & 823.91 & 216.85 & $9.56 \mathrm{E}+14$ & $42.77 \%$ & $2.29 \mathrm{E}+21$ \\
\hline & A-18 & -16.70 & 10228.90 & 770.46 & 203.95 & $8.78 \mathrm{E}+14$ & $38.87 \%$ & $2.06 \mathrm{E}+21$ \\
\hline & A-19 & -18.76 & 9242.57 & 691.33 & 184.29 & $7.56 \mathrm{E}+14$ & $34.15 \%$ & $1.79 \mathrm{E}+21$ \\
\hline & A-20 & -20.82 & 7867.16 & 583.79 & 156.86 & $6.12 \mathrm{E}+14$ & $28.46 \%$ & $1.48 \mathrm{E}+21$ \\
\hline & $\overline{\mathrm{MAX}}$ & NA & 10297.21 & 1035.76 & 269.07 & $1.16 \mathrm{E}+15$ & $51.87 \%$ & (3.63E+21 \\
\hline & MIN & NA & 7867.16 & 517.52 & 140.09 & $5.01 \mathrm{E}+14$ & $23.32 \%$ & $1.48 \mathrm{E}+21$ \\
\hline & AVG & NA & 12456.50 & 855.56 & 224.58 & $9.66 \mathrm{E}+14$ & $42.41 \%$ & $2.63 \mathrm{E}+21$ \\
\hline
\end{tabular}

(a) Elevations for MCNP cells a re reported at cell centroids relative to the modeled core axial midplane at 24.0 inches.

Table 17 Cycle 142A, MCNP-Calculated HGR, Neutron Flux, Depletion, and Fission Density for AFIP-2 Fuel Foils, 48.0 EFPD (EOC), Center Lobe Power@24.7 MW (80.4 EFPD Cumulative). ${ }^{3}$

\begin{tabular}{|c|c|c|c|c|c|c|c|c|}
\hline $\begin{array}{c}\text { Plate } \\
\text { ID }\end{array}$ & Cell & $\begin{array}{l}\text { MCNP Cell } \\
\text { Elevation }^{\text {(a) }} \\
\text { (inches) }^{\text {(inch }}\end{array}$ & $\begin{array}{c}\text { Fission } \\
\text { Power Density } \\
(\mathrm{W} / \mathrm{cc})\end{array}$ & $\begin{array}{c}\text { Fission } \\
\text { Heat Rate } \\
(\mathrm{W} / \mathrm{g})\end{array}$ & $\begin{array}{c}\text { Surface } \\
\text { Heat Flux } \\
\left(\mathrm{W} / \mathrm{cm}^{2}\right)\end{array}$ & $\begin{array}{c}\text { Neutron } \\
\text { Flux } \\
\left(\mathrm{n} / \mathrm{cm}^{2} \mathrm{sec}\right) \\
\end{array}$ & $\begin{array}{c}\% \text { Depletion } \\
\text { U-235 } \\
(\%)\end{array}$ & $\begin{array}{c}\text { Fission } \\
\text { Density } \\
\text { (fissions/cc) }\end{array}$ \\
\hline \multirow{10}{*}{$2 \mathrm{TT}$} & $\mathrm{A}-1$ & 20.82 & 8299.14 & 511.26 & 137.02 & $5.15 \mathrm{E}+14$ & $29.11 \%$ & $1.98 \mathrm{E}+21$ \\
\hline & A-2 & 18.76 & 9975.38 & 621.63 & 164.69 & $6.72 \mathrm{E}+14$ & $36.22 \%$ & $2.50 \mathrm{E}+21$ \\
\hline & A-3 & 16.70 & 11322.63 & 712.93 & 186.94 & $8.19 \mathrm{E}+14$ & $42.46 \%$ & $2.97 \mathrm{E}+21$ \\
\hline & A-4 & 14.64 & 12167.86 & 772.54 & 200.89 & $9.28 \mathrm{E}+14$ & $47.42 \%$ & $3.36 \mathrm{E}+21$ \\
\hline & A-5 & 12.58 & 12805.42 & 818.91 & 211.42 & $1.02 \mathrm{E}+15$ & $51.68 \%$ & $3.70 \mathrm{E}+21$ \\
\hline & A-6 & 10.52 & 13055.87 & 838.95 & 215.55 & $1.08 \mathrm{E}+15$ & $54.39 \%$ & $3.93 \mathrm{E}+21$ \\
\hline & A-7 & 8.46 & 13203.21 & 851.44 & 217.98 & $1.12 \mathrm{E}+15$ & $56.34 \%$ & $4.09 \mathrm{E}+21$ \\
\hline & A- 8 & 6.40 & 13302.62 & 860.46 & 219.63 & $1.15 \mathrm{E}+15$ & $58.14 \%$ & $4.25 \mathrm{E}+21$ \\
\hline & A-9 & 4.34 & 13551.17 & 877.92 & 223.73 & $1.17 \mathrm{E}+15$ & $59.13 \%$ & $4.33 \mathrm{E}+21$ \\
\hline & A-10 & 2.28 & 13259.86 & 860.48 & 218.92 & $1.17 \mathrm{E}+15$ & $59.92 \%$ & $4.40 \mathrm{E}+21$ \\
\hline \multirow{13}{*}{$2 \mathrm{BZ}$} & $\overline{\mathrm{A}-11}$ & -2.28 & 1010336.11 & 808.74 & 206.09 & $=1.18 \mathrm{E}+15$ & $61.62 \%$ & $3.45 \mathrm{E}+21$ \\
\hline & A-12 & -4.34 & 10156.39 & 793.26 & 202.51 & $1.16 \mathrm{E}+15$ & $60.36 \%$ & $3.37 \mathrm{E}+21$ \\
\hline & A-13 & -6.40 & 10035.89 & 782.85 & 200.11 & $1.15 \mathrm{E}+15$ & $59.60 \%$ & $3.32 \mathrm{E}+21$ \\
\hline & A-14 & -8.46 & 10073.53 & 784.75 & 200.86 & $1.13 \mathrm{E}+15$ & $58.81 \%$ & $3.27 \mathrm{E}+21$ \\
\hline & A-15 & -10.52 & 9906.56 & 769.68 & 197.53 & $1.10 \mathrm{E}+15$ & $57.21 \%$ & $3.16 \mathrm{E}+21$ \\
\hline & A-16 & -12.58 & 9715.02 & 751.84 & 193.71 & $1.05 \mathrm{E}+15$ & $54.74 \%$ & $3.00 \mathrm{E}+21$ \\
\hline & A-17 & -14.64 & 9557.31 & 736.02 & 190.56 & $9.80 \mathrm{E}+14$ & $51.69 \%$ & $2.81 \mathrm{E}+21$ \\
\hline & A-18 & -16.70 & 9027.19 & 690.54 & 179.99 & $8.89 \mathrm{E}+14$ & $47.40 \%$ & $2.55 \mathrm{E}+21$ \\
\hline & A-19 & -18.76 & 8402.09 & 637.37 & 167.53 & $7.72 \mathrm{E}+14$ & $42.05 \%$ & $2.23 \mathrm{E}+21$ \\
\hline & A-20 & -20.82 & 7389.82 & 555.01 & 147.35 & $6.29 \mathrm{E}+14$ & $35.36 \%$ & $1.85 \mathrm{E}+21$ \\
\hline & MAX & NA & 1313551.17 & 877.92 & 223.73 & 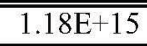 & $61.62 \%$ & 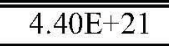 \\
\hline & MIN & NA & 7389.82 & 511.26 & 137.02 & $5.15 \mathrm{E}+14$ & $29.11 \%$ & $1.85 \mathrm{E}+21$ \\
\hline & $\mathrm{AVG}$ & NA & 10777.15 & 751.83 & 194.15 & $9.83 \mathrm{E}+14$ & $51.18 \%$ & $3.23 \mathrm{E}+21$ \\
\hline
\end{tabular}

(a) Elevations for MCNP cells a re reported at cell centroids relative to the modeled core axial midplane at 24.0 inches. 
Table 18 Cycle 142B, MCNP-Calculated HGR and Neutron Flux for AFIP-2 Fuel Foils, 0 EFPD (BOC), Center Lobe Power@25.4 MW. ${ }^{3}$

\begin{tabular}{|c|c|c|c|c|c|c|}
\hline $\begin{array}{c}\text { Plate } \\
\text { ID }\end{array}$ & Cell & $\begin{array}{c}\text { MCNP Cell } \\
\text { Elevation }^{(a)} \\
\text { (inches) }^{2}\end{array}$ & $\begin{array}{c}\text { Fission } \\
\text { Power Density } \\
\text { (W/cc) }\end{array}$ & $\begin{array}{c}\text { Fission } \\
\text { Heat Rate } \\
(\mathrm{W} / \mathrm{g})\end{array}$ & $\begin{array}{c}\text { Surface } \\
\text { Heat Flux } \\
\left(\mathrm{W} / \mathrm{cm}^{2}\right)\end{array}$ & $\begin{array}{c}\text { Neutron } \\
\text { Flux } \\
\left(\mathrm{n} / \mathrm{cm}^{2} \mathrm{sec}\right)\end{array}$ \\
\hline & A-1 & 20.82 & 6110.72 & 375.97 & 100.89 & $4.63 \mathrm{E}+14$ \\
& A-2 & 18.76 & 7483.53 & 465.58 & 123.55 & $6.04 \mathrm{E}+14$ \\
& A-3 & 16.70 & 8538.67 & 536.59 & 140.97 & $7.46 \mathrm{E}+14$ \\
& A-4 & 14.64 & 9241.78 & 585.48 & 152.58 & $8.51 \mathrm{E}+14$ \\
& A-5 & 12.58 & 9781.69 & 624.14 & 161.50 & $9.52 \mathrm{E}+14$ \\
& A-6 & 10.52 & 10348.65 & 663.42 & 170.86 & $1.02 \mathrm{E}+15$ \\
& A-7 & 8.46 & 10741.84 & 690.99 & 177.35 & $1.08 \mathrm{E}+15$ \\
& A-8 & 6.40 & 10877.46 & 701.90 & 179.59 & $1.12 \mathrm{E}+15$ \\
& A-9 & 4.34 & 11065.64 & 715.20 & 182.69 & $1.14 \mathrm{E}+15$ \\
& A-10 & 2.28 & 11427.68 & 739.69 & 188.67 & $1.15 \mathrm{E}+15$ \\
\hline \hline & A-11 & -2.28 & 8613.55 & 671.89 & 171.75 & $1.15 \mathrm{E}+15$ \\
& A-12 & -4.34 & 8560.25 & 666.50 & 170.68 & $1.16 \mathrm{E}+15$ \\
& A-13 & -6.40 & 8484.73 & 659.73 & 169.18 & $1.14 \mathrm{E}+15$ \\
& A-14 & -8.46 & 8398.94 & 652.31 & 167.47 & $1.12 \mathrm{E}+15$ \\
ABZ & A-15 & -10.52 & 8173.23 & 633.01 & 162.97 & $1.07 \mathrm{E}+15$ \\
& A-16 & -12.58 & 7955.37 & 613.84 & 158.62 & $1.02 \mathrm{E}+15$ \\
& A-17 & -14.64 & 7667.93 & 588.78 & 152.89 & $9.36 \mathrm{E}+14$ \\
& A-18 & -16.70 & 7157.07 & 545.98 & 142.70 & $8.40 \mathrm{E}+14$ \\
& A-19 & -18.76 & 6560.23 & 496.38 & 130.80 & $7.18 \mathrm{E}+14$ \\
& A-20 & -20.82 & 5708.32 & 427.78 & 113.82 & $5.76 \mathrm{E}+14$ \\
\hline \hline & MAX & NA & 11427.68 & 739.69 & 188.67 & $1.16 \mathrm{E}+15$ \\
\hline & MIN & NA & 5708.32 & 375.97 & 100.89 & $4.63 \mathrm{E}+14$ \\
\hline & AVG & NA & 8644.86 & 602.76 & 155.98 & $9.42 \mathrm{E}+14$ \\
\hline
\end{tabular}

(a) Elevations for MCNP cells a re reported at cell centroids relative to the modeled core axial midplane at 24.0 inches.

Table 19 Cycle 142B, MCNP-Calculated HGR, Neutron Flux, Depletion, and Fission Density for AFIP-2 Fuel Foils, 16.0 EFPD (MOC1), Center Lobe Power@ 25.4 MW (96.4 EFPD Cumulative). ${ }^{3}$

\begin{tabular}{|c|c|c|c|c|c|c|c|c|}
\hline $\begin{array}{c}\text { Plate } \\
\text { ID }\end{array}$ & Cell & $\begin{array}{l}\text { MCNP Cell } \\
\text { Elevation }^{(a)} \\
\text { (inches) }^{\text {(inche }}\end{array}$ & $\begin{array}{c}\text { Fission } \\
\text { Power Density } \\
\text { (W/cc) }\end{array}$ & $\begin{array}{c}\text { Fission } \\
\text { Heat Rate } \\
(\mathrm{W} / \mathrm{g})\end{array}$ & $\begin{array}{c}\text { Surface } \\
\text { Heat Flux } \\
\left(\mathrm{W} / \mathrm{cm}^{2}\right)\end{array}$ & $\begin{array}{c}\text { Neutron } \\
\text { Flux } \\
\left(\mathrm{n} / \mathrm{cm}^{2} \mathrm{sec}\right) \\
\end{array}$ & $\begin{array}{c}\% \text { Depletion } \\
\text { U-235 } \\
(\%)\end{array}$ & $\begin{array}{c}\text { Fission } \\
\text { Density } \\
\text { (fissions/cc) }\end{array}$ \\
\hline \multirow{10}{*}{$2 \mathrm{TT}$} & $\mathrm{A}-1$ & 20.82 & 6233.29 & 385.98 & 102.91 & $4.77 \mathrm{E}+14$ & $33.20 \%$ & $2.27 \mathrm{E}+21$ \\
\hline & A-2 & 18.76 & 7411.87 & 464.77 & 122.37 & $6.24 \mathrm{E}+14$ & $41.10 \%$ & $2.85 \mathrm{E}+21$ \\
\hline & A-3 & 16.70 & 8548.85 & 542.03 & 141.14 & $7.71 \mathrm{E}+14$ & $47.88 \%$ & $3.38 \mathrm{E}+21$ \\
\hline & A-4 & 14.64 & 9154.90 & 585.68 & 151.15 & $8.76 \mathrm{E}+14$ & $53.16 \%$ & $3.80 \mathrm{E}+21$ \\
\hline & A-5 & 12.58 & 9542.21 & 615.21 & 157.54 & $9.65 \mathrm{E}+14$ & $57.64 \%$ & $4.16 \mathrm{E}+21$ \\
\hline & A-6 & 10.52 & 9896.56 & 641.45 & 163.39 & $1.03 \mathrm{E}+15$ & $60.59 \%$ & $4.41 \mathrm{E}+21$ \\
\hline & A-7 & 8.46 & 10054.42 & 654.15 & 166.00 & $1.07 \mathrm{E}+15$ & $62.68 \%$ & $4.60 \mathrm{E}+21$ \\
\hline & A- 8 & 6.40 & 10246.83 & 668.91 & 169.18 & $1.11 \mathrm{E}+15$ & $64.49 \%$ & $4.76 \mathrm{E}+21$ \\
\hline & A-9 & 4.34 & 10062.98 & 658.05 & 166.14 & $1.11 \mathrm{E}+15$ & $65.56 \%$ & $4.85 \mathrm{E}+21$ \\
\hline & A-10 & 2.28 & 10302.96 & 674.96 & 170.10 & $1.12 \mathrm{E}+15$ & $66.50 \%$ & $4.94 \mathrm{E}+21$ \\
\hline \multirow{13}{*}{$2 \mathrm{BZ}$} & A-11 & -2.28 & 77735.25 & 610.11 & 154.23 & $1.12 \mathrm{E}+15$ & $68.10 \%$ & $3.86 \mathrm{E}+21$ \\
\hline & A-12 & -4.34 & 7758.16 & 610.75 & 154.69 & $1.12 \mathrm{E}+15$ & $66.82 \%$ & $3.77 \mathrm{E}+21$ \\
\hline & A-13 & -6.40 & 7782.47 & 611.72 & 155.17 & $1.12 \mathrm{E}+15$ & $66.04 \%$ & $3.71 \mathrm{E}+21$ \\
\hline & A-14 & -8.46 & 7757.07 & 608.98 & 154.67 & $1.10 \mathrm{E}+15$ & $65.20 \%$ & $3.66 \mathrm{E}+21$ \\
\hline & A-15 & -10.52 & 7510.72 & 587.81 & 149.76 & $1.05 \mathrm{E}+15$ & $63.53 \%$ & $3.54 \mathrm{E}+21$ \\
\hline & A-16 & -12.58 & 7448.88 & 580.53 & 148.52 & $1.01 \mathrm{E}+15$ & $60.99 \%$ & $3.38 \mathrm{E}+21$ \\
\hline & A-17 & -14.64 & 7128.65 & 552.81 & 142.14 & $9.28 \mathrm{E}+14$ & $57.81 \%$ & $3.17 \mathrm{E}+21$ \\
\hline & A-18 & -16.70 & 6898.40 & 531.13 & 137.55 & $8.46 \mathrm{E}+14$ & $53.23 \%$ & $2.89 \mathrm{E}+21$ \\
\hline & A-19 & -18.76 & 6268.65 & 478.37 & 124.99 & $7.23 \mathrm{E}+14$ & $47.56 \%$ & $2.54 \mathrm{E}+21$ \\
\hline & A-20 & -20.82 & 5696.32 & 429.98 & 113.58 & $5.84 \mathrm{E}+14$ & $40.28 \%$ & $2.12 \mathrm{E}+21$ \\
\hline & MAX & NA & 1010302.96 & 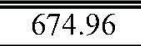 & $\overline{170.10}$ & $1.12 \mathrm{E}+15$ & $68.10 \%$ & $24.94 \mathrm{E}+21$ \\
\hline & MIN & NA & 5696.32 & 385.98 & 102.91 & $4.77 \mathrm{E}+14$ & $33.20 \%$ & $2.12 \mathrm{E}+21$ \\
\hline & $\overline{A V G}$ & $\mathrm{NA}$ & 8171.97 & 574.67 & 147.26 & $9.38 \mathrm{E}+14$ & $57.12 \%$ & $3.63 \mathrm{E}+21$ \\
\hline
\end{tabular}

(a) Elevations for MCNP cells a re reported at cell centroids relative to the modeled core axial midplane at 24.0 inches. 
Table 20 Cycle 142B, MCNP-Calculated HGR, Neutron Flux, Depletion, and Fission Density for AFIP-2 Fuel Foils, 29.7 EFPD (MOC2), Center Lobe Power@ 25.4 MW (110.1 EFPD Cumulative). ${ }^{3}$

\begin{tabular}{|c|c|c|c|c|c|c|c|c|}
\hline $\begin{array}{c}\text { Plate } \\
\text { ID }\end{array}$ & Cell & $\begin{array}{l}\text { MCNP Cell } \\
\text { Elevation }^{(a)} \\
\text { (inches) }^{\text {inch }}\end{array}$ & $\begin{array}{c}\text { Fission } \\
\text { Power Density } \\
\text { (W/cc) }\end{array}$ & $\begin{array}{c}\text { Fission } \\
\text { Heat Rate } \\
(\mathrm{W} / \mathrm{g})\end{array}$ & $\begin{array}{c}\text { Surface } \\
\text { Heat Flux } \\
\left(\mathrm{W} / \mathrm{cm}^{2}\right)\end{array}$ & $\begin{array}{c}\text { Neutron } \\
\text { Flux } \\
\left(\mathrm{n} / \mathrm{cm}^{2} \mathrm{sec}\right)\end{array}$ & $\begin{array}{c}\% \text { Depletion } \\
\text { U-235 } \\
(\%)\end{array}$ & $\begin{array}{c}\text { Fission } \\
\text { Density } \\
\text { (fissions/cc) }\end{array}$ \\
\hline \multirow{10}{*}{$2 \mathrm{TT}$} & A-1 & 20.82 & 7305.96 & 454.96 & 120.62 & $5.31 \mathrm{E}+14$ & $36.76 \%$ & $2.52 \mathrm{E}+21$ \\
\hline & A-2 & 18.76 & 8537.82 & 539.01 & 140.96 & $6.92 \mathrm{E}+14$ & $45.21 \%$ & $3.15 \mathrm{E}+21$ \\
\hline & A-3 & 16.70 & 9599.57 & 613.46 & 158.49 & $8.41 \mathrm{E}+14$ & $52.47 \%$ & $3.72 \mathrm{E}+21$ \\
\hline & A-4 & 14.64 & 10162.74 & 655.65 & 167.79 & $9.62 \mathrm{E}+14$ & $57.95 \%$ & $4.17 \mathrm{E}+21$ \\
\hline & A-5 & 12.58 & 10557.48 & 686.65 & 174.30 & $1.05 \mathrm{E}+15$ & $62.50 \%$ & $4.55 \mathrm{E}+21$ \\
\hline & A-6 & 10.52 & 10817.34 & 707.63 & 178.59 & $1.13 \mathrm{E}+15$ & $65.52 \%$ & $4.81 \mathrm{E}+21$ \\
\hline & A-7 & 8.46 & 10819.88 & 710.54 & 178.64 & $1.16 \mathrm{E}+15$ & $67.61 \%$ & $5.00 \mathrm{E}+21$ \\
\hline & A- 8 & 6.40 & 10945.45 & 721.32 & 180.71 & $1.20 \mathrm{E}+15$ & $69.46 \%$ & $5.17 \mathrm{E}+21$ \\
\hline & A-9 & 4.34 & 10844.16 & 715.90 & 179.04 & $1.21 \mathrm{E}+15$ & $70.43 \%$ & $5.25 \mathrm{E}+21$ \\
\hline & A-10 & 2.28 & 10990.90 & 727.02 & 181.46 & $1.22 \mathrm{E}+15$ & $71.45 \%$ & $5.35 \mathrm{E}+21$ \\
\hline \multirow{13}{*}{$2 \mathrm{BZ}$} & $\overline{\mathrm{A}-11}$ & -2.28 & 8235.38 & 655.20 & 164.21 & $1.22 \mathrm{E}+15$ & $\overline{72.93 \%}$ & $4.17 \mathrm{E}+21$ \\
\hline & A-12 & -4.34 & 8382.14 & 665.52 & 167.13 & $1.21 \mathrm{E}+15$ & $71.67 \%$ & $4.08 \mathrm{E}+21$ \\
\hline & A-13 & -6.40 & 8414.53 & 667.12 & 167.78 & $1.21 \mathrm{E}+15$ & $70.94 \%$ & $4.03 E+21$ \\
\hline & A-14 & -8.46 & 8417.02 & 666.54 & 167.83 & $1.19 \mathrm{E}+15$ & $70.12 \%$ & $3.98 \mathrm{E}+21$ \\
\hline & A-15 & -10.52 & 8159.42 & 643.96 & 162.69 & $1.14 \mathrm{E}+15$ & $68.37 \%$ & $3.85 \mathrm{E}+21$ \\
\hline & A-16 & -12.58 & 8137.74 & 639.49 & 162.26 & $1.09 \mathrm{E}+15$ & $65.88 \%$ & $3.68 \mathrm{E}+21$ \\
\hline & A-17 & -14.64 & 7958.84 & 622.01 & 158.69 & $1.02 \mathrm{E}+15$ & $62.58 \%$ & $3.46 \mathrm{E}+21$ \\
\hline & A- 18 & -16.70 & 7663.93 & 594.60 & 152.81 & $9.23 \mathrm{E}+14$ & $57.96 \%$ & $3.17 \mathrm{E}+21$ \\
\hline & A-19 & -18.76 & 7134.39 & 548.14 & 142.25 & $7.95 \mathrm{E}+14$ & $52.00 \%$ & $2.80 \mathrm{E}+21$ \\
\hline & A-20 & -20.82 & 6428.10 & 488.24 & 128.17 & $6.45 \mathrm{E}+14$ & $44.46 \%$ & $2.35 \mathrm{E}+21$ \\
\hline & MAX & NA & 10990.90 & $\overline{7727.02}$ & $\bar{~} 181.46$ & $1.22 \mathrm{E}+15$ & $7 \overline{72.93 \%}$ & 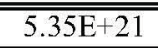 \\
\hline & MIN & NA & 6428.10 & 454.96 & 120.62 & $5.31 \mathrm{E}+14$ & $36.76 \%$ & $2.35 \mathrm{E}+21$ \\
\hline & AVG & NA & 8975.64 & 636.15 & 161.72 & $1.02 \mathrm{E}+15$ & $61.81 \%$ & $3.96 \mathrm{E}+21$ \\
\hline
\end{tabular}

(a) Elevations for MCNP cells a re reported at cell centroids relative to the modeled core axial midplane at 24.0 inches.

Table 21 Cycle 142B, MCNP-Calculated HGR, Neutron Flux, Depletion, and Fission Density for AFIP-2 Fuel Foils, 52.0 EFPD (EOC), Center Lobe Power@ 25.4 MW (132.4 EFPD Cumulative). ${ }^{3}$

\begin{tabular}{|c|c|c|c|c|c|c|c|c|}
\hline $\begin{array}{c}\text { Plate } \\
\text { ID }\end{array}$ & Cell & $\begin{array}{l}\text { MCNP Cell } \\
\text { Elevation }^{(a)} \\
\text { (inches) }\end{array}$ & $\begin{array}{c}\text { Fission } \\
\text { Power Density } \\
\text { (W/cc) }\end{array}$ & $\begin{array}{c}\text { Fission } \\
\text { Heat Rate } \\
(\mathrm{W} / \mathrm{g})\end{array}$ & $\begin{array}{c}\text { Surface } \\
\text { Heat Flux } \\
\left(\mathrm{W} / \mathrm{cm}^{2}\right) \\
\end{array}$ & $\begin{array}{c}\text { Neutron } \\
\text { Flux } \\
\left(\mathrm{n} / \mathrm{cm}^{2} \mathrm{sec}\right) \\
\end{array}$ & $\begin{array}{c}\% \text { Depletion } \\
\text { U-235 } \\
(\%)\end{array}$ & $\begin{array}{c}\text { Fission } \\
\text { Density } \\
\text { (fissions/cc) } \\
\end{array}$ \\
\hline \multirow{10}{*}{$2 \mathrm{TT}$} & A-1 & 20.82 & 6861.53 & 431.95 & 113.28 & $5.29 \mathrm{E}+14$ & $43.44 \%$ & $3.01 \mathrm{E}+21$ \\
\hline & A-2 & 18.76 & 7844.20 & 501.49 & 129.51 & $6.88 \mathrm{E}+14$ & $52.70 \%$ & $3.73 \mathrm{E}+21$ \\
\hline & A-3 & 16.70 & 8604.32 & 557.78 & 142.06 & $8.38 \mathrm{E}+14$ & $60.51 \%$ & $4.37 \mathrm{E}+21$ \\
\hline & A-4 & 14.64 & 8934.20 & 585.20 & 147.50 & $9.54 \mathrm{E}+14$ & $66.14 \%$ & $4.85 \mathrm{E}+21$ \\
\hline & A-5 & 12.58 & 9124.25 & 602.85 & 150.64 & $1.04 \mathrm{E}+15$ & $70.73 \%$ & $5.26 \mathrm{E}+21$ \\
\hline & A-6 & 10.52 & 9226.58 & 613.27 & 152.33 & $1.12 \mathrm{E}+15$ & $73.71 \%$ & $5.54 \mathrm{E}+21$ \\
\hline & A-7 & 8.46 & 9137.28 & 609.78 & 150.86 & $1.15 \mathrm{E}+15$ & $75.63 \%$ & $5.73 \mathrm{E}+21$ \\
\hline & A- 8 & 6.40 & 9126.46 & 611.28 & 150.68 & $1.18 \mathrm{E}+15$ & $77.43 \%$ & $5.90 \mathrm{E}+21$ \\
\hline & A-9 & 4.34 & 9073.70 & 608.77 & 149.81 & $1.20 \mathrm{E}+15$ & $78.25 \%$ & $5.98 \mathrm{E}+21$ \\
\hline & A-10 & 2.28 & 9044.86 & 608.09 & 149.33 & $1.21 \mathrm{E}+15$ & $79.30 \%$ & $6.09 \mathrm{E}+21$ \\
\hline \multirow{13}{*}{$2 \mathrm{BZ}$} & A-11 & -2.28 & 6733.26 & "543.55 & 134.25 & $1.21 \mathrm{E}+15$ & $80.57 \%$ & $4.72 \mathrm{E}+21$ \\
\hline & A-12 & -4.34 & 6916.62 & 557.41 & 137.91 & $1.21 \mathrm{E}+15$ & $79.46 \%$ & $4.65 \mathrm{E}+21$ \\
\hline & A-13 & -6.40 & 6974.93 & 561.28 & 139.07 & $1.20 \mathrm{E}+15$ & $78.85 \%$ & $4.59 \mathrm{E}+21$ \\
\hline & A-14 & -8.46 & 6974.34 & 560.56 & 139.06 & $1.17 \mathrm{E}+15$ & $78.04 \%$ & $4.54 \mathrm{E}+21$ \\
\hline & A-15 & -10.52 & 6872.93 & 550.43 & 137.04 & $1.13 \mathrm{E}+15$ & $76.27 \%$ & $4.39 \mathrm{E}+21$ \\
\hline & A-16 & -12.58 & 6907.16 & 550.70 & 137.72 & $1.09 \mathrm{E}+15$ & $73.99 \%$ & $4.22 \mathrm{E}+21$ \\
\hline & A-17 & -14.64 & 6875.24 & 545.03 & 137.09 & $1.01 \mathrm{E}+15$ & $70.72 \%$ & $3.99 \mathrm{E}+21$ \\
\hline & A-18 & -16.70 & 6745.72 & 530.50 & 134.50 & $9.17 \mathrm{E}+14$ & $66.09 \%$ & $3.68 \mathrm{E}+21$ \\
\hline & A-19 & -18.76 & 6423.32 & 499.92 & 128.07 & $7.91 \mathrm{E}+14$ & $59.90 \%$ & $3.28 \mathrm{E}+21$ \\
\hline & A-20 & -20.82 & 5915.91 & 454.53 & 117.96 & $6.42 \mathrm{E}+14$ & $51.92 \%$ & $2.78 \mathrm{E}+21$ \\
\hline & MAX & $\mathrm{NA}$ & 9226.58 & 613.27 & 152.33 & $1.21 \mathrm{E}+15$ & $80.57 \%$ & $6.09 \mathrm{E}+21$ \\
\hline & MIN & NA & 5915.91 & 431.95 & 113.28 & $5.29 \mathrm{E}+14$ & $43.44 \%$ & $2.78 \mathrm{E}+21$ \\
\hline & AVG & NA & 7715.84 & 554.22 & 138.93 & $1.01 \mathrm{E}+15$ & $69.68 \%$ & $4.56 \mathrm{E}+21$ \\
\hline
\end{tabular}

(a) Elevations for MCNP cells a re reported at cell centroids rela tive to the modeled core axial midpla ne at 24.0 inches 
Table 22. Corrected fission power density (W/cc) and fission dens ity (fissions/cc) for ATR Cycle 141A.

\begin{tabular}{|c|c|c|c|c|c|c|c|c|}
\hline \multirow[b]{2}{*}{$\begin{array}{l}\text { Plate } \\
\text { ID }\end{array}$} & \multirow[b]{2}{*}{ Cell } & BOC 141A & \multicolumn{2}{|c|}{ MOC1 141A } & \multicolumn{2}{|c|}{ MOC2 141A } & \multicolumn{2}{|c|}{ EOC 141A } \\
\hline & & $\begin{array}{c}\text { Fission } \\
\text { Power Density } \\
\text { (W/cc) }\end{array}$ & $\begin{array}{l}\text { Fission } \\
\text { Power } \\
\text { Density } \\
\text { (W/cc) }\end{array}$ & $\begin{array}{c}\text { Fission } \\
\text { Density } \\
\text { (fissions/cc) }\end{array}$ & $\begin{array}{l}\text { Fission } \\
\text { Power } \\
\text { Density } \\
\text { (W/cc) }\end{array}$ & $\begin{array}{c}\text { Fission } \\
\text { Density } \\
\text { (fissions/cc) }\end{array}$ & $\begin{array}{l}\text { Fission } \\
\text { Power } \\
\text { Density } \\
\text { (W/cc) }\end{array}$ & $\begin{array}{c}\text { Fission } \\
\text { Density } \\
\text { (fissions/cc) }\end{array}$ \\
\hline \multirow{10}{*}{$2 \mathrm{TT}$} & A-1 & 8032.77 & 7943.11 & $3.575 \mathrm{E}+20$ & 7799.27 & $5.934 \mathrm{E}+20$ & 7680.50 & $7.642 \mathrm{E}+20$ \\
\hline & A-2 & 10469.10 & 10238.85 & $4.661 \mathrm{E}+20$ & 9944.91 & $7.698 \mathrm{E}+20$ & 9733.90 & $9.843 \mathrm{E}+20$ \\
\hline & A-3 & 12462.81 & 12346.86 & $5.553 \mathrm{E}+20$ & 11933.67 & $9.211 \mathrm{E}+20$ & 11627.58 & $1.179 \mathrm{E}+21$ \\
\hline & A-4 & 14384.44 & 14009.43 & $6.407 \mathrm{E}+20$ & 13458.29 & $1.059 \mathrm{E}+21$ & 13070.48 & $1.346 \mathrm{E}+21$ \\
\hline & A-5 & 16443.38 & 15510.51 & $7.326 \mathrm{E}+20$ & 14869.39 & $1.189 \mathrm{E}+21$ & 14388.03 & $1.514 \mathrm{E}+21$ \\
\hline & A-6 & 17515.19 & 16520.53 & $7.800 \mathrm{E}+20$ & 15791.53 & $1.272 \mathrm{E}+21$ & 15206.44 & $1.616 \mathrm{E}+21$ \\
\hline & A-7 & 18579.92 & 17532.11 & $8.274 \mathrm{E}+20$ & 16642.58 & $1.346 \mathrm{E}+21$ & 16002.91 & $1.709 \mathrm{E}+21$ \\
\hline & A-8 & 19247.91 & 17949.79 & $8.571 \mathrm{E}+20$ & 17018.47 & $1.393 \mathrm{E}+21$ & 16348.94 & $1.764 \mathrm{E}+21$ \\
\hline & A-9 & 19659.17 & 18421.62 & $8.756 \mathrm{E}+20$ & 17452.74 & $1.421 \mathrm{E}+21$ & 16718.98 & $1.801 \mathrm{E}+21$ \\
\hline & A- 10 & 20198.88 & 18914.10 & $8.998 \mathrm{E}+20$ & 17939.79 & $1.458 \mathrm{E}+21$ & 17173.31 & $1.857 \mathrm{E}+21$ \\
\hline \multirow{10}{*}{$2 \mathrm{BZ}$} & A-11 & 22845.78 & 20962.93 & $1.018 \mathrm{E}+21$ & 19683.68 & $1.635 \mathrm{E}+21$ & 18766.89 & $2.067 \mathrm{E}+21$ \\
\hline & A-12 & 22139.38 & 20233.13 & $9.865 \mathrm{E}+20$ & 19134.22 & $1.583 \mathrm{E}+21$ & 18288.35 & $2.002 \mathrm{E}+21$ \\
\hline & A-13 & 21517.56 & 20249.86 & $9.590 \mathrm{E}+20$ & 19079.52 & $1.557 \mathrm{E}+21$ & 18217.31 & $1.976 \mathrm{E}+21$ \\
\hline & A-14 & 21151.16 & 19833.39 & $9.420 \mathrm{E}+20$ & 18825.61 & $1.531 \mathrm{E}+21$ & 17982.68 & $1.936 \mathrm{E}+21$ \\
\hline & A-15 & 20408.99 & 19110.34 & $9.093 \mathrm{E}+20$ & 18135.80 & $1.478 \mathrm{E}+21$ & 17392.42 & $1.871 \mathrm{E}+21$ \\
\hline & A-16 & 18996.95 & 17924.73 & $8.465 \mathrm{E}+20$ & 17063.06 & $1.374 \mathrm{E}+21$ & 16382.31 & $1.753 \mathrm{E}+21$ \\
\hline & A-17 & 17578.79 & 16683.47 & $7.837 \mathrm{E}+20$ & 15952.76 & $1.278 \mathrm{E}+21$ & 15401.22 & $1.622 \mathrm{E}+21$ \\
\hline & A- 18 & 15570.96 & 15082.47 & $6.934 \mathrm{E}+20$ & 14471.78 & $1.141 \mathrm{E}+21$ & 14042.76 & $1.452 \mathrm{E}+21$ \\
\hline & A-19 & 13323.63 & 13034.02 & $5.940 \mathrm{E}+20$ & 12607.70 & $9.799 \mathrm{E}+20$ & 12285.04 & $1.256 \mathrm{E}+21$ \\
\hline & A-20 & 10795.96 & 10579.08 & $4.815 \mathrm{E}+20$ & 10301.10 & $7.942 \mathrm{E}+20$ & 10109.36 & $1.021 \mathrm{E}+21$ \\
\hline
\end{tabular}


Table 23. Corrected fission power density (W/cc) and fission density (fissions/cc) for ATR Cycle 142A.

\begin{tabular}{|c|c|c|c|c|c|c|c|c|}
\hline \multirow[b]{2}{*}{$\begin{array}{l}\text { Plate } \\
\text { ID }\end{array}$} & \multirow[b]{2}{*}{ Cell } & \multirow{2}{*}{$\begin{array}{c}\text { BOC 142A } \\
\begin{array}{c}\text { Fission } \\
\text { Power Density } \\
(W / c c)\end{array}\end{array}$} & \multicolumn{2}{|c|}{ MOC1 142A } & \multicolumn{2}{|c|}{ MOC2 142A } & \multicolumn{2}{|c|}{ EOC 142A } \\
\hline & & & $\begin{array}{l}\text { Fission } \\
\text { Power } \\
\text { Density } \\
\text { (W/cc) }\end{array}$ & $\begin{array}{c}\text { Fission } \\
\text { Density } \\
\text { (fissions/cc) }\end{array}$ & $\begin{array}{l}\text { Fission } \\
\text { Power } \\
\text { Density } \\
(\mathrm{W} / \mathrm{cc} \text { ) }\end{array}$ & $\begin{array}{c}\text { Fission } \\
\text { Density } \\
\text { (fissions/cc) }\end{array}$ & $\begin{array}{l}\text { Fission } \\
\text { Power } \\
\text { Density } \\
\text { (W/cc) }\end{array}$ & $\begin{array}{c}\text { Fission } \\
\text { Density } \\
\text { (fissions/cc) }\end{array}$ \\
\hline \multirow{10}{*}{$2 \mathrm{TT}$} & A-1 & 7071.64 & 7746.50 & $1.096 \mathrm{E}+21$ & 7879.28 & $1.467 \mathrm{E}+21$ & 7706.34 & $1.839 \mathrm{E}+21$ \\
\hline & A-2 & 8888.24 & 9565.86 & $1.402 \mathrm{E}+21$ & 9709.05 & $1.857 \mathrm{E}+21$ & 9262.85 & $2.321 \mathrm{E}+21$ \\
\hline & A-3 & 10650.03 & 11285.63 & $1.681 \mathrm{E}+21$ & 11392.96 & $2.219 \mathrm{E}+21$ & 10513.87 & $2.758 \mathrm{E}+21$ \\
\hline & A-4 & 12222.26 & 12599.28 & $1.922 \mathrm{E}+21$ & 12607.21 & $2.526 \mathrm{E}+21$ & 11298.73 & $3.120 \mathrm{E}+21$ \\
\hline & A-5 & 13298.54 & 13675.74 & $2.145 \mathrm{E}+21$ & 13573.61 & $2.786 \mathrm{E}+21$ & 11890.75 & $3.436 \mathrm{E}+21$ \\
\hline & A-6 & 14390.30 & 14306.91 & $2.294 \mathrm{E}+21$ & 14225.37 & $2.971 \mathrm{E}+21$ & 12123.31 & $3.649 \mathrm{E}+21$ \\
\hline & A-7 & 15052.33 & 14761.20 & $2.414 \mathrm{E}+21$ & 14337.90 & $3.120 \mathrm{E}+21$ & 12260.12 & $3.798 \mathrm{E}+21$ \\
\hline & A-8 & 15794.06 & 15333.22 & $2.507 \mathrm{E}+21$ & 14948.12 & $3.231 \mathrm{E}+21$ & 12352.43 & $3.946 \mathrm{E}+21$ \\
\hline & A-9 & 16047.01 & 15636.15 & $2.554 \mathrm{E}+21$ & 15133.12 & $3.296 \mathrm{E}+21$ & 12583.23 & $4.021 \mathrm{E}+21$ \\
\hline & A- 10 & 16416.64 & 15698.18 & $2.619 \mathrm{E}+21$ & 15046.70 & $3.371 \mathrm{E}+21$ & 12312.73 & $4.086 \mathrm{E}+21$ \\
\hline \multirow{10}{*}{$2 \mathrm{BZ}$} & A-11 & 17974.64 & 17318.55 & $2.918 \mathrm{E}+21$ & 16424.58 & $3.729 \mathrm{E}+21$ & 13523.08 & $4.514 \mathrm{E}+21$ \\
\hline & A-12 & 17685.11 & 16991.30 & $2.839 \mathrm{E}+21$ & 16147.06 & $3.637 \mathrm{E}+21$ & 13287.94 & $4.409 \mathrm{E}+21$ \\
\hline & A-13 & 17384.57 & 16669.38 & $2.787 \mathrm{E}+21$ & 15844.57 & $3.585 \mathrm{E}+21$ & 13130.29 & $4.344 \mathrm{E}+21$ \\
\hline & A-14 & 17126.42 & 16517.19 & $2.748 \mathrm{E}+21$ & 15706.69 & $3.533 \mathrm{E}+21$ & 13179.54 & $4.278 \mathrm{E}+21$ \\
\hline & A-15 & 16455.14 & 15982.52 & $2.643 \mathrm{E}+21$ & 15374.08 & $3.402 \mathrm{E}+21$ & 12961.08 & $4.134 \mathrm{E}+21$ \\
\hline & A-16 & 15688.55 & 15414.60 & $2.486 \mathrm{E}+21$ & 14899.93 & $3.219 \mathrm{E}+21$ & 12710.48 & $3.925 \mathrm{E}+21$ \\
\hline & A-17 & 14615.42 & 14511.24 & $2.316 \mathrm{E}+21$ & 14229.11 & $2.996 \mathrm{E}+21$ & 12504.15 & $3.676 \mathrm{E}+21$ \\
\hline & A- 18 & 13208.68 & 13147.08 & $2.080 \mathrm{E}+21$ & 13382.81 & $2.695 \mathrm{E}+21$ & 11810.57 & $3.336 \mathrm{E}+21$ \\
\hline & A-19 & 11493.32 & 11610.83 & $1.792 \mathrm{E}+21$ & 12092.36 & $2.342 \mathrm{E}+21$ & 10992.73 & $2.918 \mathrm{E}+21$ \\
\hline & A- 20 & 9509.15 & 9841.72 & $1.465 \mathrm{E}+21$ & 10292.87 & $1.936 \mathrm{E}+21$ & 9668.35 & $2.420 \mathrm{E}+21$ \\
\hline
\end{tabular}


Table 24. Corrected fission power density (W/cc) and fission density (fissions/cc) for ATR Cycle 142B.

\begin{tabular}{|c|c|c|c|c|c|c|c|c|}
\hline \multirow[b]{2}{*}{$\begin{array}{l}\text { Plate } \\
\text { ID }\end{array}$} & \multirow[b]{2}{*}{ Cell } & \multirow{2}{*}{$\begin{array}{c}\text { BOC 142B } \\
\text { Fission } \\
\text { Power Density } \\
(W / c c)\end{array}$} & \multicolumn{2}{|c|}{ MOC1 142B } & \multicolumn{2}{|c|}{ MOC2 142B } & \multicolumn{2}{|c|}{ EOC 142B } \\
\hline & & & $\begin{array}{l}\text { Fission } \\
\text { Power } \\
\text { Density } \\
\text { (W/cc) }\end{array}$ & $\begin{array}{c}\text { Fission } \\
\text { Density } \\
\text { (fissions/cc) }\end{array}$ & $\begin{array}{l}\text { Fission } \\
\text { Power } \\
\text { Density } \\
\text { (W/cc) }\end{array}$ & $\begin{array}{c}\text { Fission } \\
\text { Density } \\
\text { (fissions/cc) }\end{array}$ & $\begin{array}{c}\text { Fission } \\
\text { Power } \\
\text { Density } \\
(W / \mathrm{cc})\end{array}$ & $\begin{array}{c}\text { Fission } \\
\text { Density } \\
\text { (fissions/cc) }\end{array}$ \\
\hline \multirow{10}{*}{$2 \mathrm{TT}$} & A-1 & 5674.24 & 5788.06 & $2.108 \mathrm{E}+21$ & 6784.11 & $2.340 \mathrm{E}+21$ & 6371.42 & $2.795 \mathrm{E}+21$ \\
\hline & A-2 & 6948.99 & 6882.45 & $2.646 \mathrm{E}+21$ & 7927.98 & $2.925 \mathrm{E}+21$ & 7283.90 & $3.464 \mathrm{E}+21$ \\
\hline & A-3 & 7928.77 & 7938.22 & $3.139 \mathrm{E}+21$ & 8913.89 & $3.454 \mathrm{E}+21$ & 7989.73 & $4.058 \mathrm{E}+21$ \\
\hline & A-4 & 8581.65 & 8500.98 & $3.529 \mathrm{E}+21$ & 9436.83 & $3.872 \mathrm{E}+21$ & 8296.04 & $4.504 \mathrm{E}+21$ \\
\hline & A-5 & 9083.00 & 8860.62 & $3.863 \mathrm{E}+21$ & 9803.37 & $4.225 \mathrm{E}+21$ & 8472.52 & $4.884 \mathrm{E}+21$ \\
\hline & A-6 & 9609.46 & 9189.66 & $4.095 \mathrm{E}+21$ & 10044.67 & $4.466 \mathrm{E}+21$ & 8567.54 & $5.144 \mathrm{E}+21$ \\
\hline & A-7 & 9974.57 & 9336.25 & $4.271 \mathrm{E}+21$ & 10047.03 & $4.643 \mathrm{E}+21$ & 8484.62 & $5.321 \mathrm{E}+21$ \\
\hline & A-8 & 10100.50 & 9514.91 & $4.420 \mathrm{E}+21$ & 10163.63 & $4.801 \mathrm{E}+21$ & 8474.57 & $5.479 \mathrm{E}+21$ \\
\hline & A-9 & 10275.24 & 9344.20 & $4.504 \mathrm{E}+21$ & 10069.58 & $4.875 \mathrm{E}+21$ & 8425.58 & $5.553 \mathrm{E}+21$ \\
\hline & A- 10 & 10611.42 & 9567.03 & $4.587 \mathrm{E}+21$ & 10205.84 & $4.968 \mathrm{E}+21$ & 8398.80 & $5.655 \mathrm{E}+21$ \\
\hline \multirow{10}{*}{$2 \mathrm{BZ}$} & A-11 & 11269.39 & 10120.29 & $5.050 \mathrm{E}+21$ & 10774.62 & $5.456 \mathrm{E}+21$ & 8809.35 & $6.175 \mathrm{E}+21$ \\
\hline & A-12 & 11199.66 & 10150.26 & $4.932 \mathrm{E}+21$ & 10966.63 & $5.338 \mathrm{E}+21$ & 9049.24 & $6.084 \mathrm{E}+21$ \\
\hline & A-13 & 11100.86 & 10182.06 & $4.854 \mathrm{E}+21$ & 11009.01 & $5.273 \mathrm{E}+21$ & 9125.53 & $6.005 \mathrm{E}+21$ \\
\hline & A-14 & 10988.61 & 10148.83 & $4.789 \mathrm{E}+21$ & 11012.27 & $5.207 \mathrm{E}+21$ & 9124.76 & $5.940 \mathrm{E}+21$ \\
\hline & A-15 & 10693.31 & 9826.53 & $4.632 \mathrm{E}+21$ & 10675.24 & $5.037 \mathrm{E}+21$ & 8992.08 & $5.744 \mathrm{E}+21$ \\
\hline & A-16 & 10408.28 & 9745.62 & $4.422 \mathrm{E}+21$ & 10646.88 & $4.815 \mathrm{E}+21$ & 9036.87 & $5.521 \mathrm{E}+21$ \\
\hline & A-17 & 10032.21 & 9326.65 & $4.147 \mathrm{E}+21$ & 10412.82 & $4.527 \mathrm{E}+21$ & 8995.11 & $5.220 \mathrm{E}+21$ \\
\hline & A-18 & 9363.83 & 9025.41 & $3.781 \mathrm{E}+21$ & 10026.98 & $4.147 \mathrm{E}+21$ & 8825.65 & $4.815 \mathrm{E}+21$ \\
\hline & A-19 & 8582.97 & 8201.48 & $3.323 \mathrm{E}+21$ & 9334.16 & $3.663 \mathrm{E}+21$ & 8403.84 & $4.291 \mathrm{E}+21$ \\
\hline & $A-20$ & 7468.39 & 7452.69 & $2.774 \mathrm{E}+21$ & 8410.10 & $3.075 \mathrm{E}+21$ & 7739.98 & $3.637 \mathrm{E}+21$ \\
\hline
\end{tabular}




\subsection{Axial Gradient}

The axial gradient is represented by the neutron flux profile and the corrected fission density profile as shown in Figure 10 and Figure 11 , respectively. Since the AFIP-2 experiment was irradiated in the CFT, the transverse (radial) profile is relatively flat across the plates and was not reported.

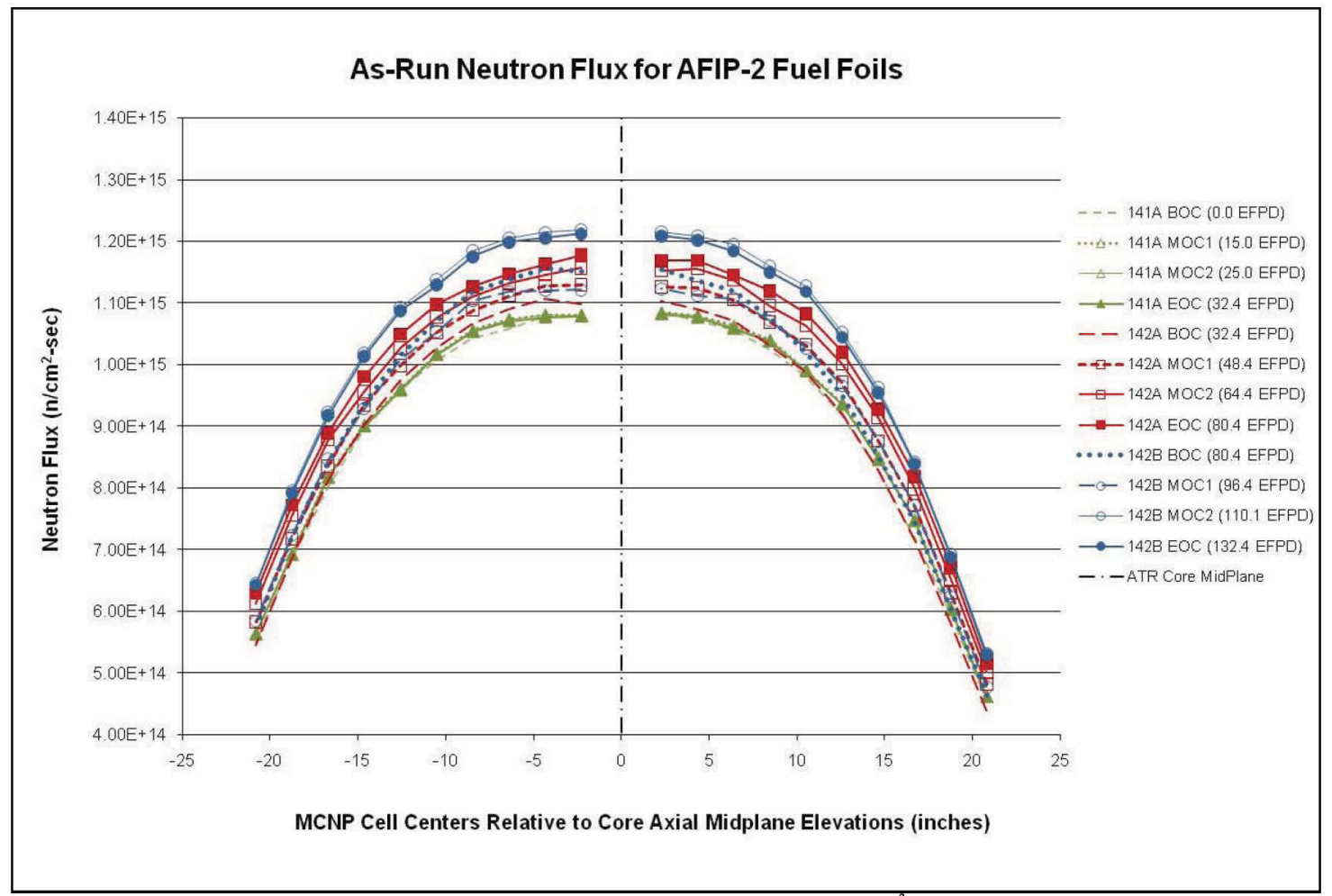

Figure 10. AFIP-2 axial neutron flux profile. ${ }^{3}$ 


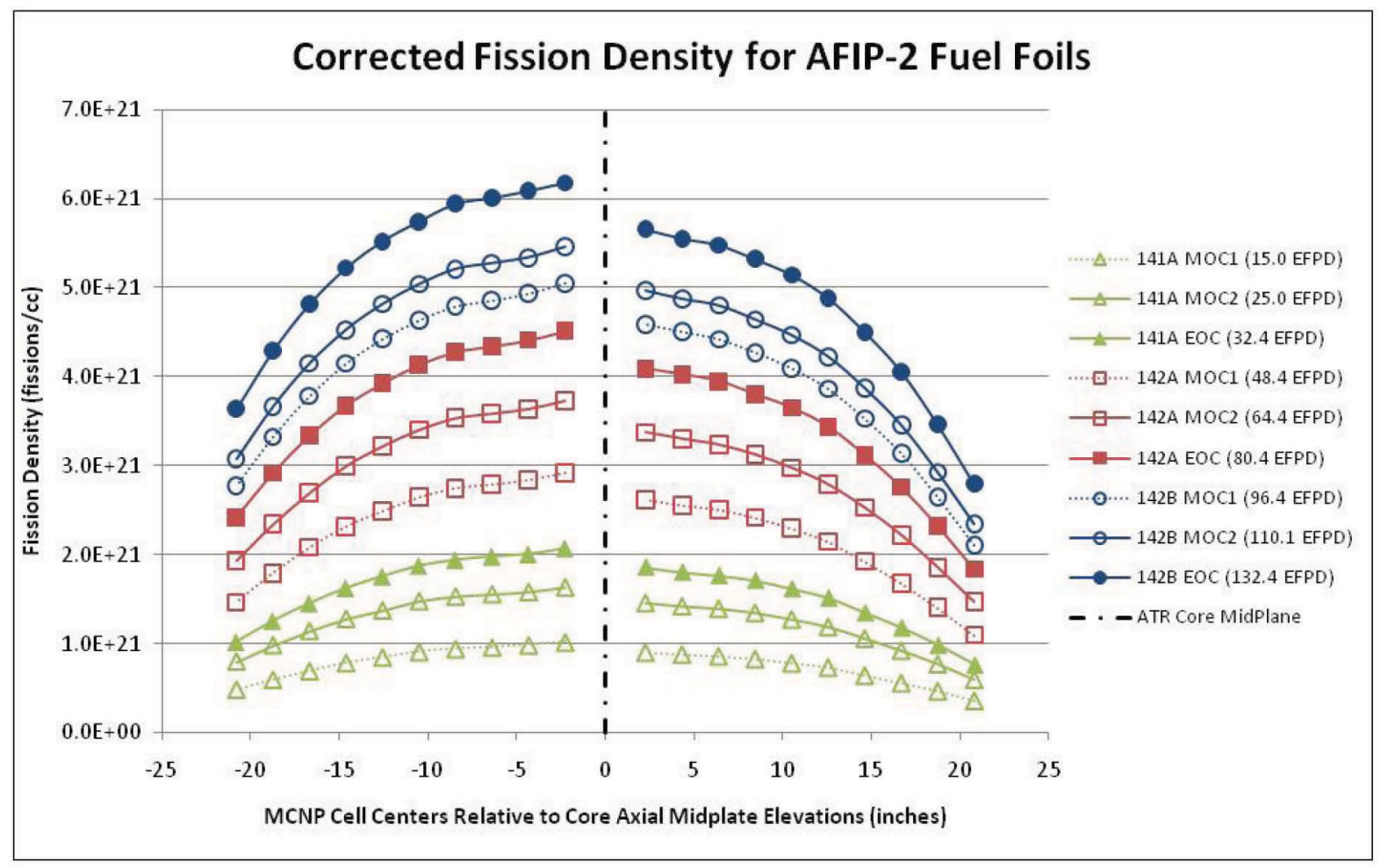

Figure 11. AFIP-2 corrected fission density axial profile. 


\section{HYDRAULIC TESTING}

Flow tests were performed to characterize the test assembly designed to irradiate full-size plates for the RERTR program in the ATR. The holder assembly design was used for several plate tests that were conducted in the CFT position?

The test apparatus was designed and constructed to simulate the ATR CFT position geometry. The holder was fabricated such that the orifice plate on the bottom of the test train could be screwed on (rather than welded) to allow variation of the orifice diameter. The results of the flow tests were used to generate estimates of the coolant velocity and flow rate and are reported in Table $25^{7}$.

Table 25. AFIP irradiation vehicle flow conditions for each orifice configuration. ${ }^{7}$

\begin{tabular}{|c|c|c|c|c|}
\hline Configuration & $\begin{array}{c}\text { Total Internal } \\
\text { Loss } \\
\text { Coefficient }\end{array}$ & $\begin{array}{c}\text { Total Holder } \\
\text { Internal Flow } \\
(\mathrm{gpm})\end{array}$ & $\begin{array}{c}\text { Channel } \\
\text { Coolant Flow } \\
(\mathrm{gpm})\end{array}$ & $\begin{array}{c}\text { Channel } \\
\text { Coolant } \\
\text { Velocity (m/s) }\end{array}$ \\
\hline $6 \mathrm{~mm}$ orifice & 0.1709 & 20.8 & 6.6 & 1.7 \\
\hline $7 \mathrm{~mm}$ orifice & 0.1203 & 24.8 & 7.9 & 2.0 \\
\hline $8 \mathrm{~mm}$ orifice & 0.0869 & 29.2 & 9.3 & 2.4 \\
\hline $8.1 \mathrm{~mm}$ orifice & 0.0861 & 29.3 & 9.3 & 2.4 \\
\hline $9 \mathrm{~mm}$ orifice & 0.0680 & 33.0 & 10.5 & 2.7 \\
\hline $10 \mathrm{~mm}$ orifice & 0.0568 & 36.1 & 11.5 & 3.0 \\
\hline Open (no orifice) & 0.00164 & 212.5 & 67.5 & 17.4 \\
\hline
\end{tabular}

Based on the results from the hydraulic testing, the orifice was sized to 0.8125 inches $(20.64 \mathrm{~mm})$ to provide a coolant channel veloc ity of approximately $34 \mathrm{ft} / \mathrm{s}$ for two pump operation ${ }^{8}$. 


\section{AS-RUN THERMAL ANALYSIS}

The thermal as-run analysis was performed using the as-built geometry, MCNP-calculated surface heat flux $\left(\mathrm{W} / \mathrm{cm}^{2}\right)$ and nominal coolant channel flow rate. Abaqus was used to calculate the coolant channel temperatures and plate surface temperatures.

\subsection{Coolant Channel Temperature}

The coolant temperature ${ }^{10}$ was analyzed at the three flow channels in the test assembly. The left coolant channel is west of plate position B, the center coolant channel is in between plate positions A and $\mathrm{B}$, and the right coolant channel is in between plate position A and the Ram Rod (refer to Figure 1). For each cycle interval, the coolant temperature was plotted as a function of location along the test assembly with 0.0 inches being at the top of the assembly. These plots are show in Figure 12 through Figure 23.

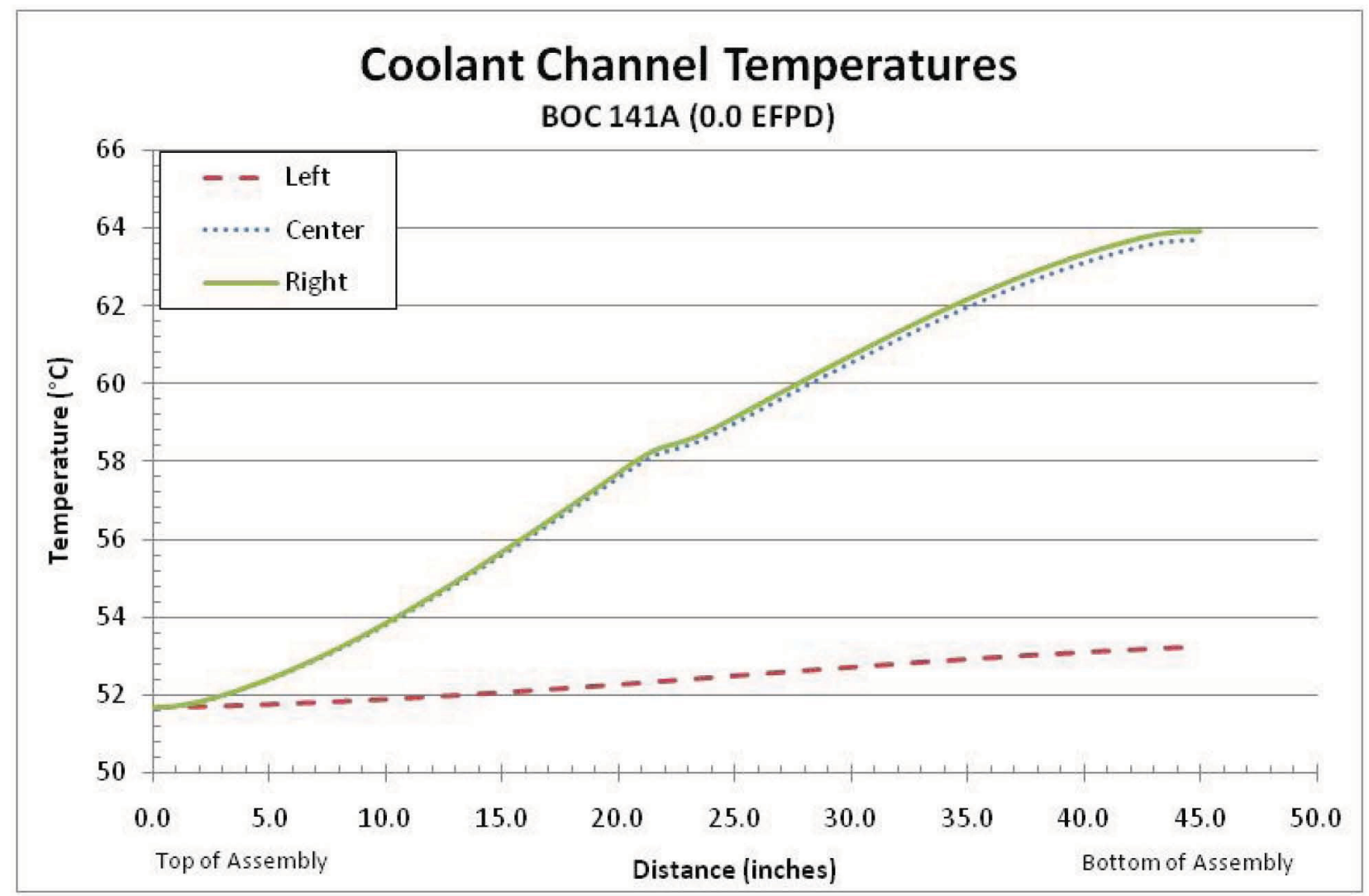

Figure 12. Coolant channel temperatures as a function of location along the test assembly at BOC 141A (0.0 EFPD). 


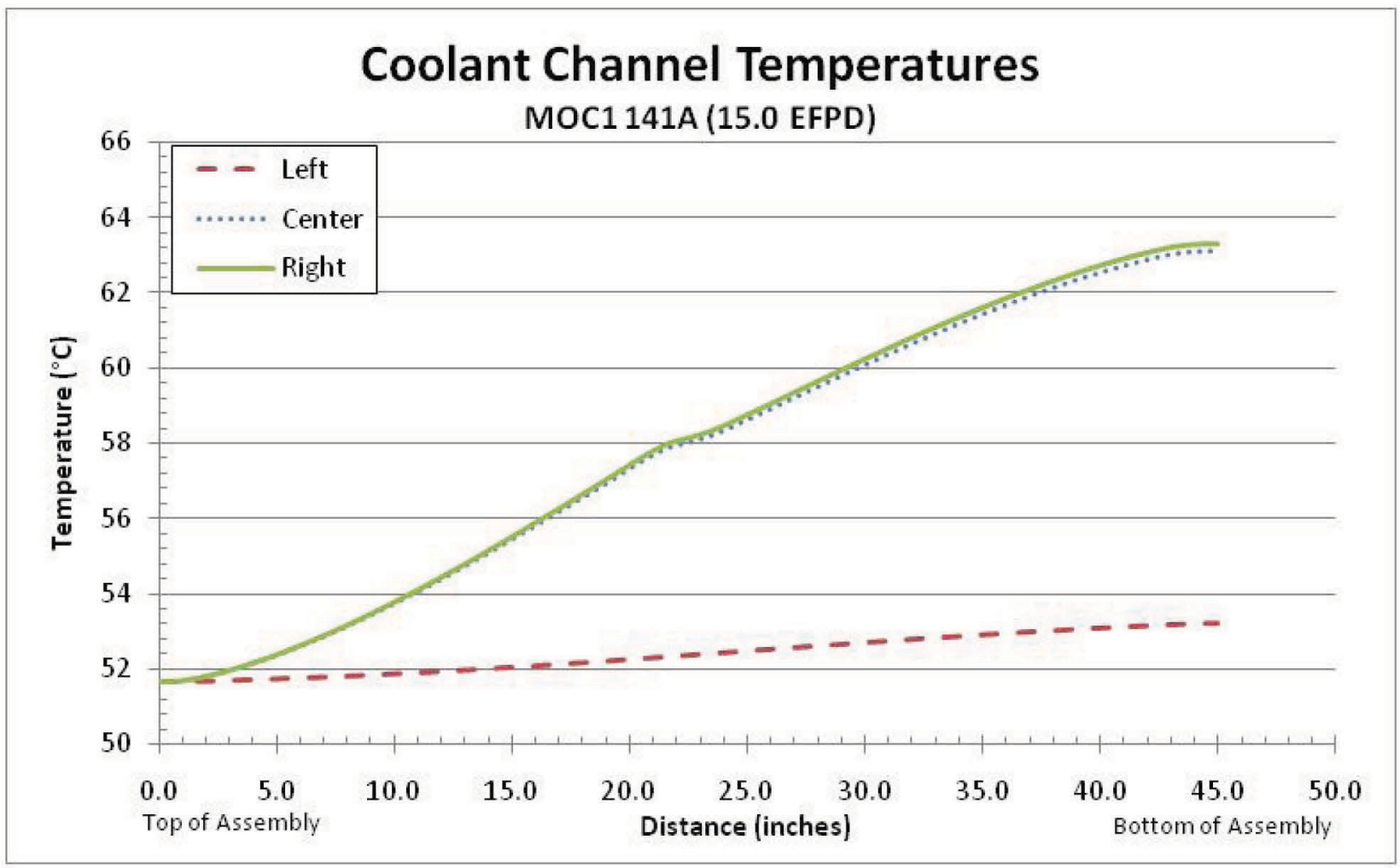

Figure 13. Coolant channel temperatures as a function of location along the test assembly at MOC 141A (15.0 EFPD).

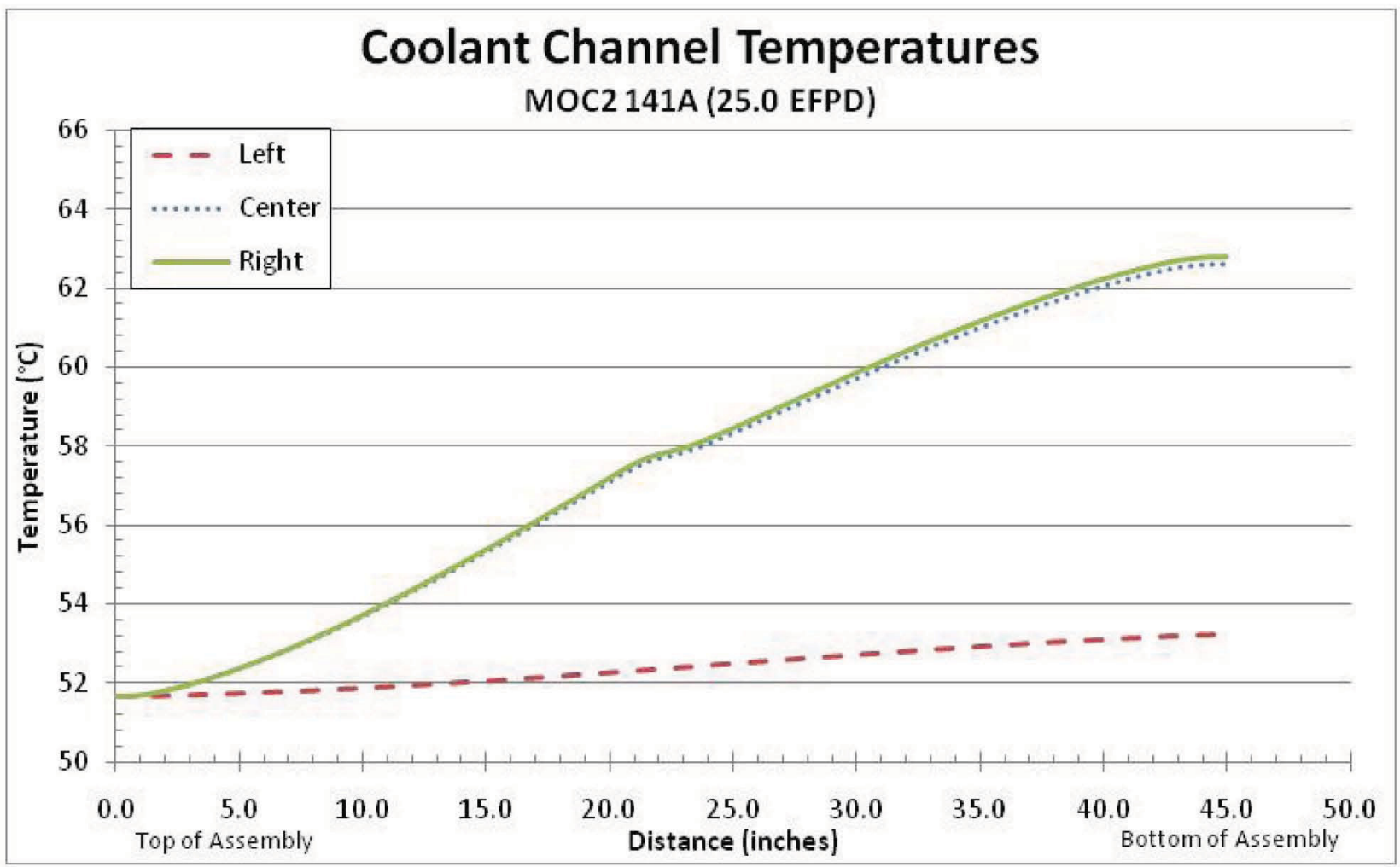

Figure 14. Coolant channel temperatures as a function of location along the test assembly at MOC 141A (25.0 EFPD). 


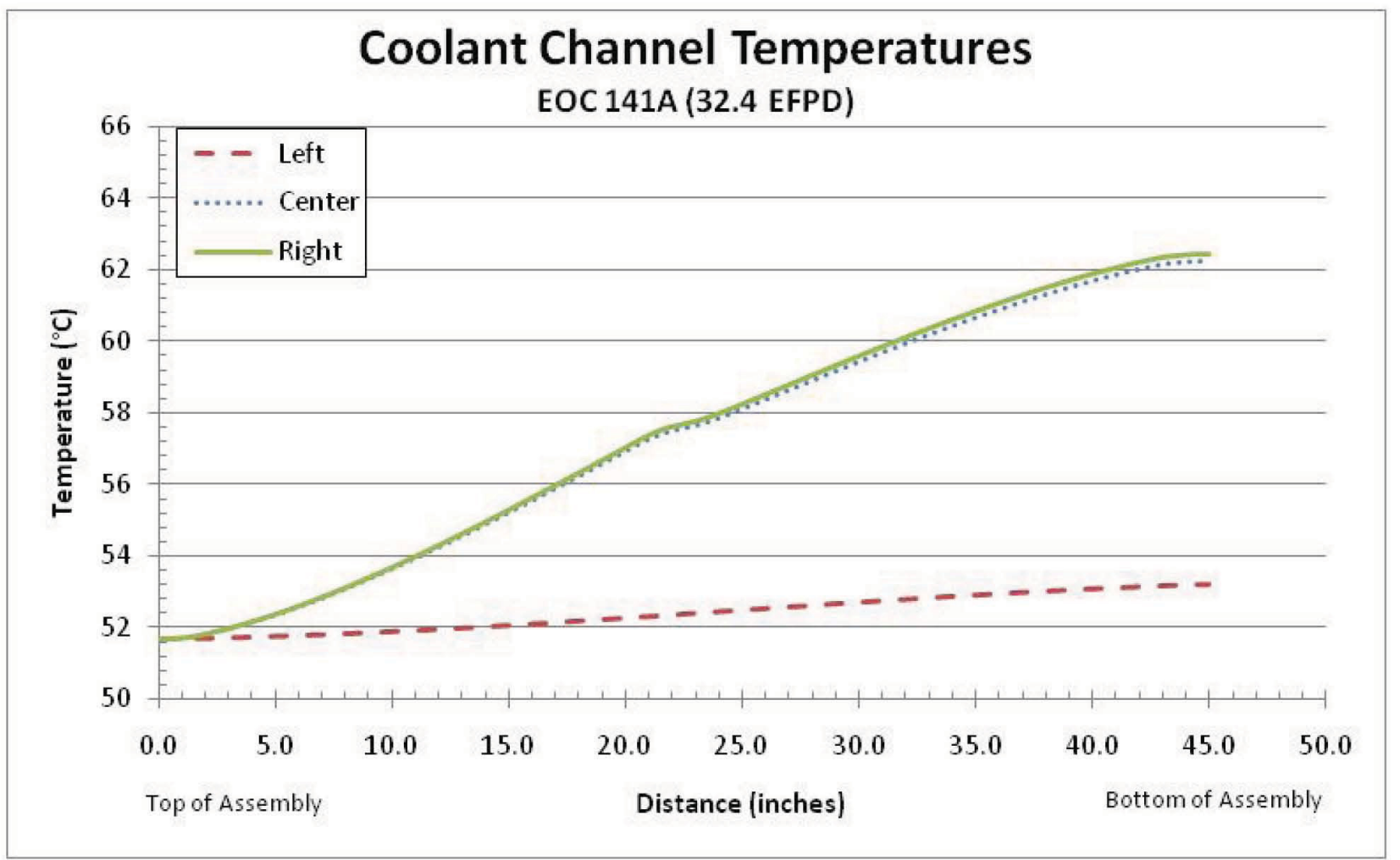

Figure 15. Coolant channel temperatures as a function of location along the test assembly at EOC 141A (32.4 EFPD).

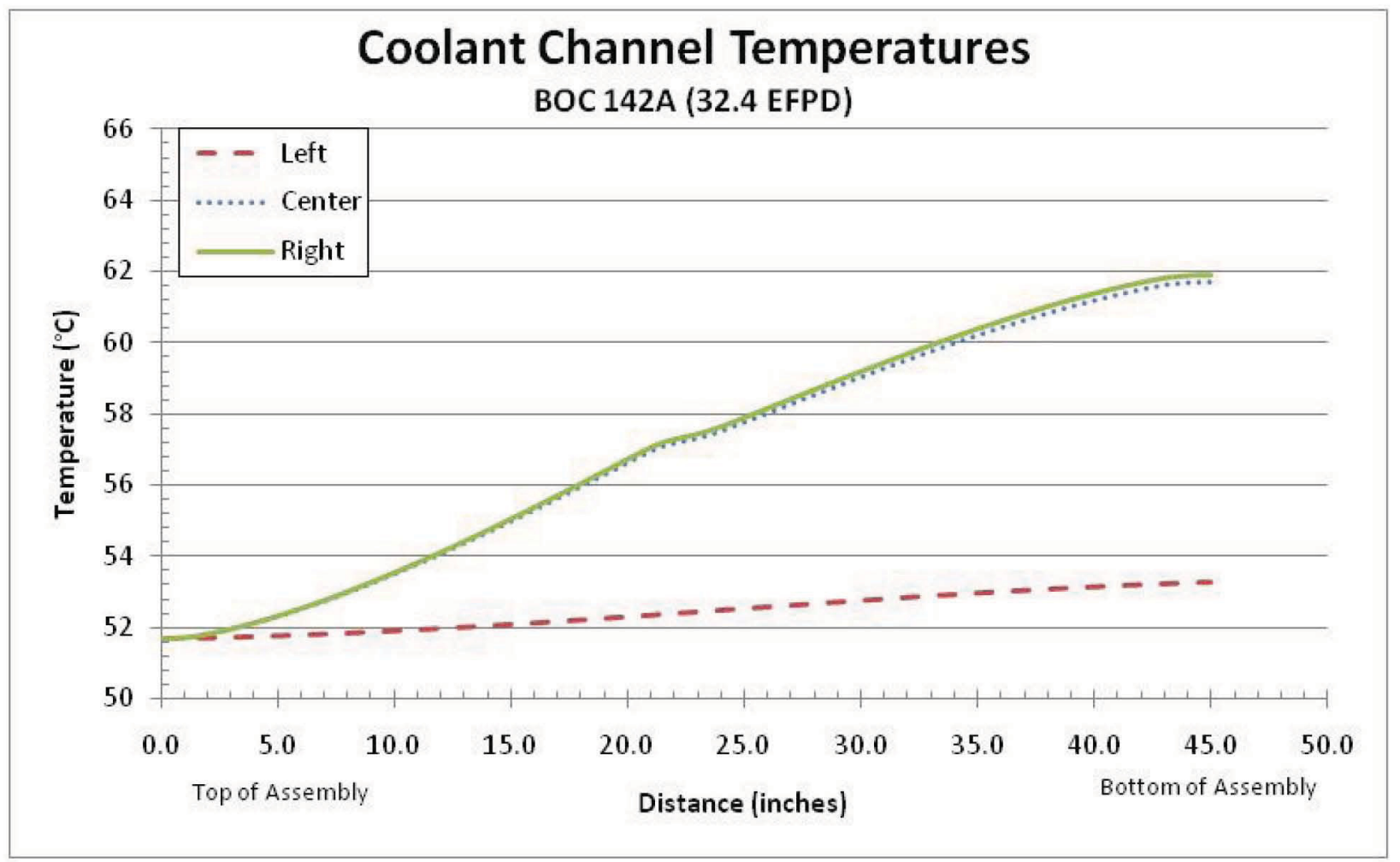

Figure 16. Coolant channel temperatures as a function of location along the test assembly at BOC 142A (32.4 EFPD). 


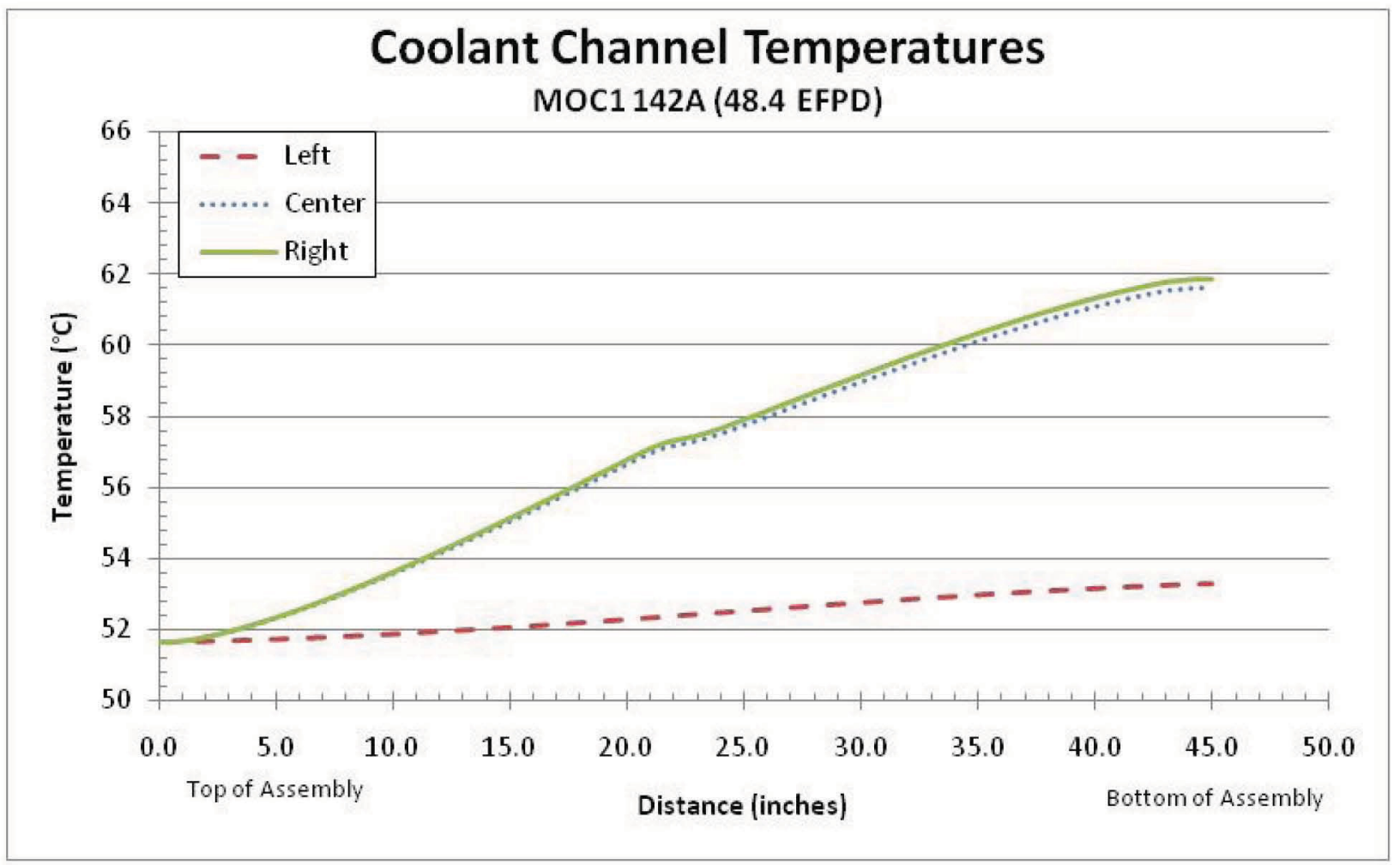

Figure 17. Coolant channel temperatures as a function of location along the test assembly at MOC 142A (48.4 EFPD).

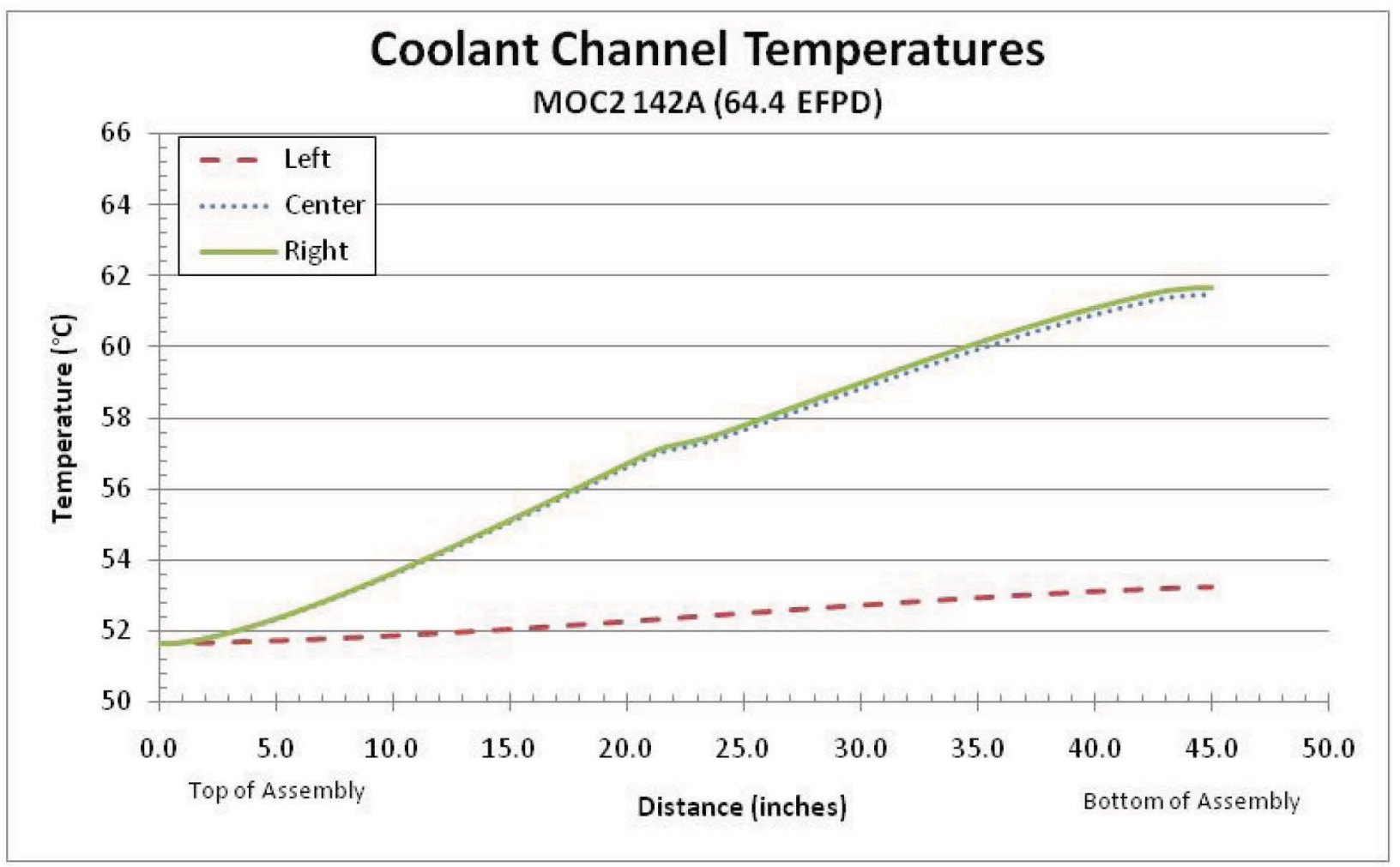

Figure 18. Coolant channel temperatures as a function of location along the test assembly at MOC 142A (64.4 EFPD). 


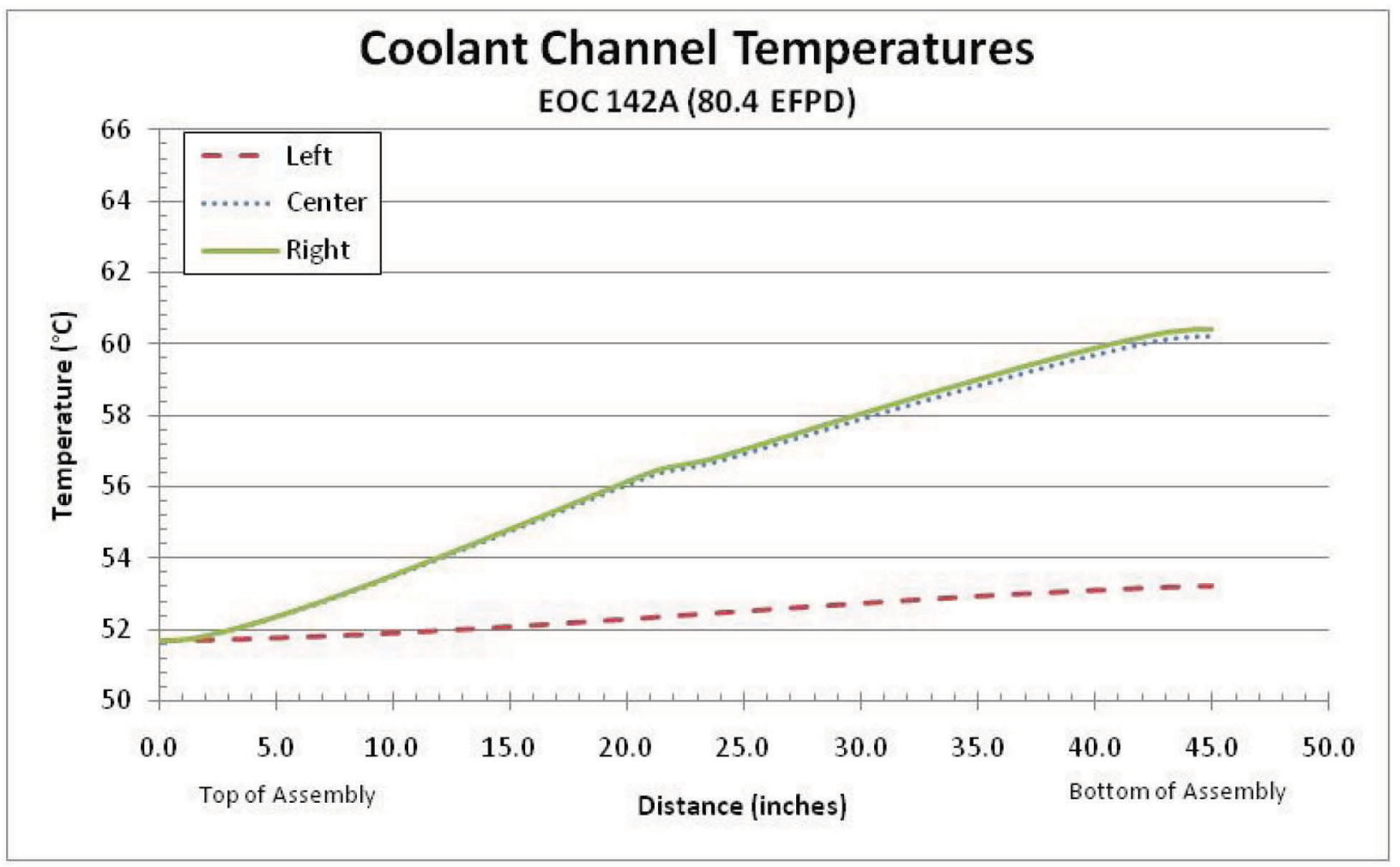

Figure 19. Coolant channel temperatures as a function of location along the test assembly at EOC 142A (80.4 EFPD).

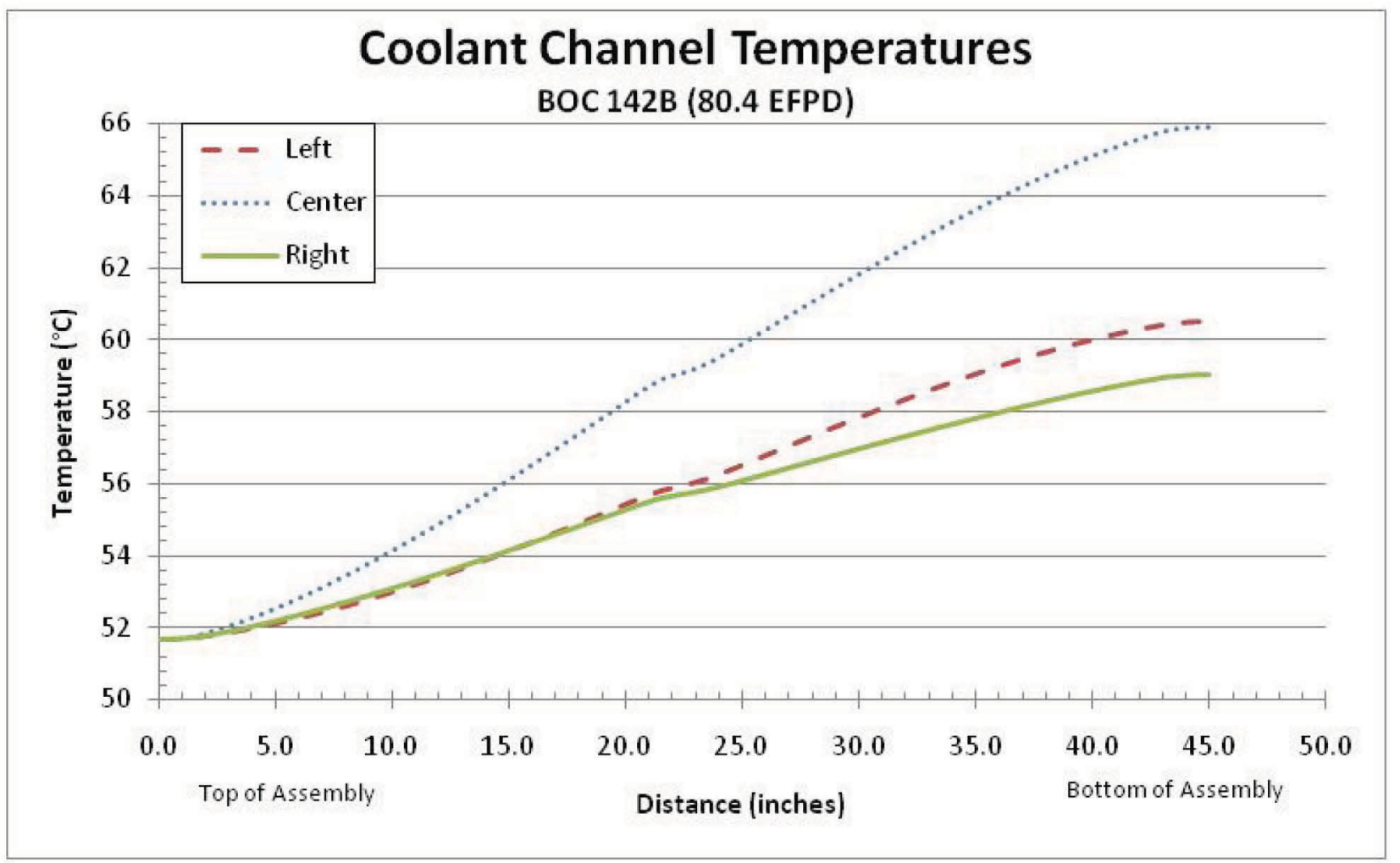

Figure 20. Coolant channel temperatures as a function of location along the test assembly at BOC $142 \mathrm{~B}$ (80.4 EFPD). NOTE: AFIP-1 was inserted into plate pos ition B and BOC 142B. 


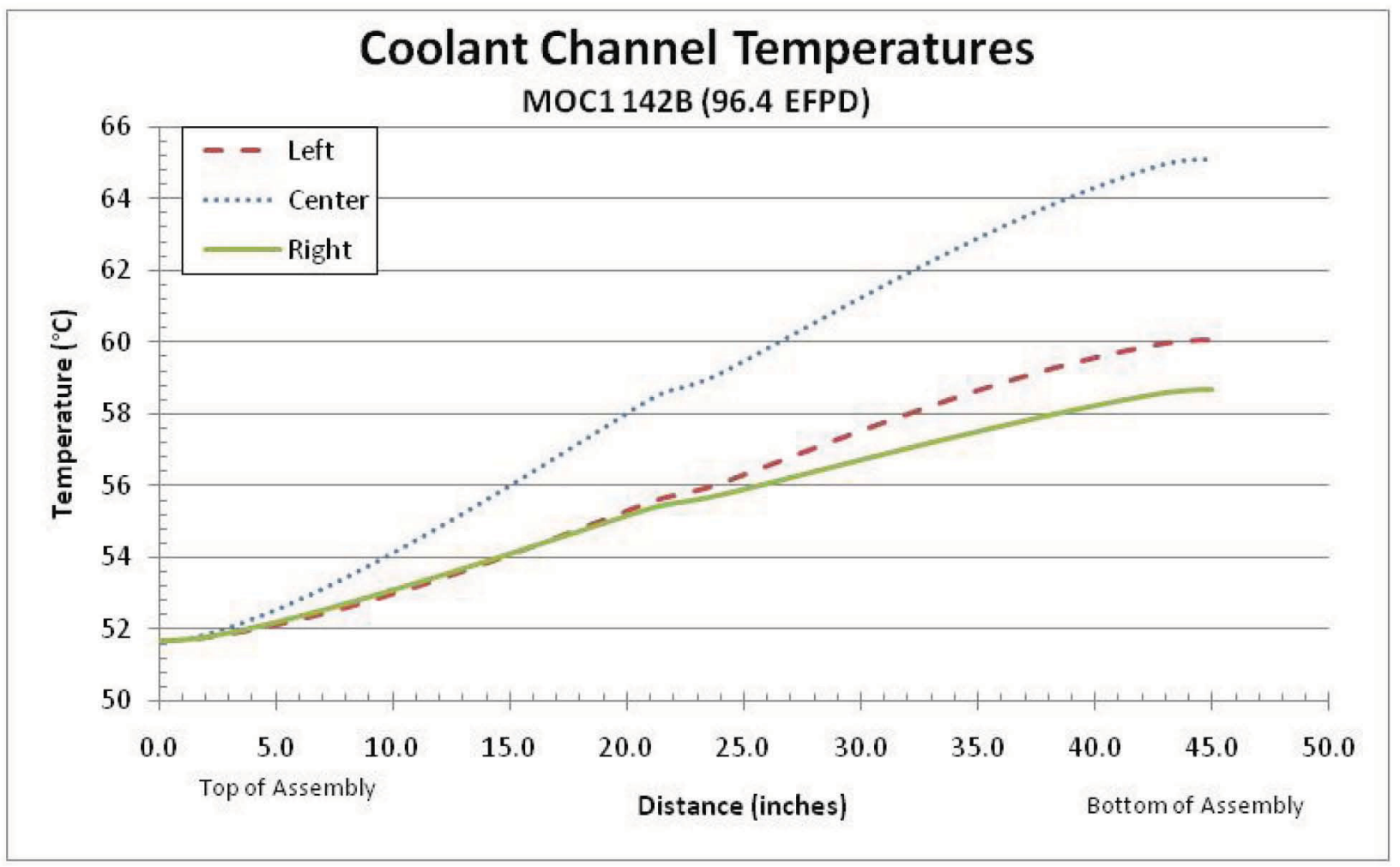

Figure 21. Coolant channel temperatures as a function of location along the test assembly at MOC 142B (96.4 EFPD).

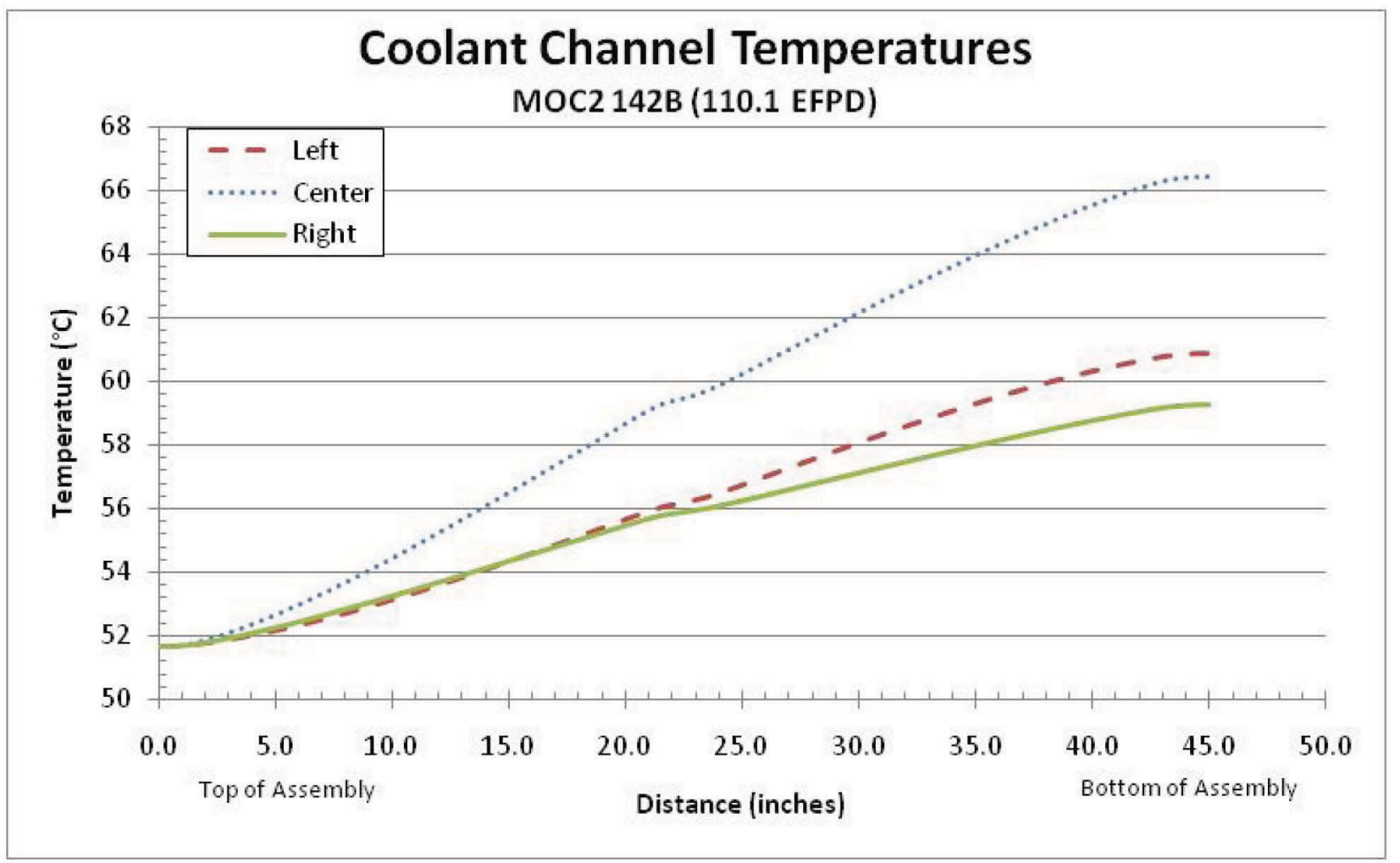

Figure 22. Coolant channel temperatures as a function of location along the test assembly at MOC 142B (110.1 EFPD). 


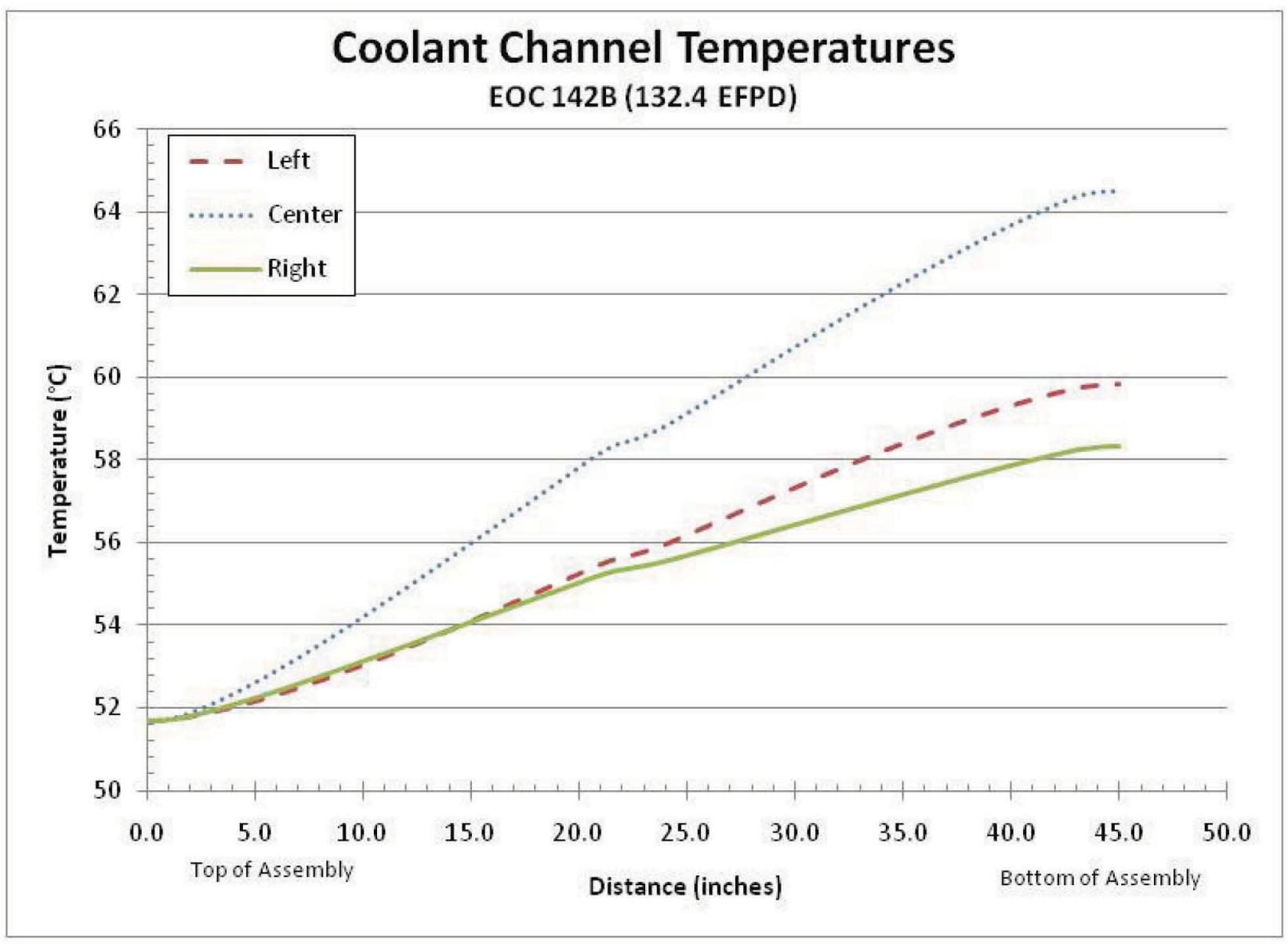

Figure 23. Coolant channel temperatures as a function of location along the test assembly at EOC 142B (132.4 EFPD). 


\subsection{Plate Surface Temperature}

The plate surface temperatures ${ }^{10}$ were analyzed at each time step for each side of the plate, with the east side of the plate facing the ram rod and west side of the plate facing plate position B. Table 26 through Table 37 tabulate the 2D map of the temperatures for each side of the plate at EOC for each cycle. The plate surface temperatures for each time step are tabulated in Appendix B.

Table 26. Temperature $\left({ }^{\circ} \mathrm{C}\right)$ map of the east side of plate 2TT at EOC 141A (32.4 EFPD).

\begin{tabular}{|c||c|c|c|c|c|c|c|c|c|c|}
\hline \multirow{2}{*}{$\begin{array}{c}\text { Length of } \\
\text { Plate (in) }\end{array}$} & 0.05 & 0.25 & 0.50 & 0.75 & 1.00 & 1.25 & 1.50 & 1.75 & 2.00 & 2.24 \\
\hline 0.125 & 51.92 & 51.77 & 51.76 & 51.76 & 51.76 & 51.76 & 51.76 & 51.77 & 51.80 & 51.93 \\
\hline 1.00 & 52.90 & 52.47 & 52.66 & 52.68 & 52.68 & 52.68 & 52.67 & 52.59 & 52.40 & 52.80 \\
\hline 2.00 & 56.67 & 81.40 & 84.41 & 84.48 & 84.48 & 84.48 & 84.47 & 84.21 & 70.99 & 55.23 \\
\hline 3.00 & 56.93 & 81.61 & 84.63 & 84.70 & 84.71 & 84.71 & 84.69 & 84.42 & 71.21 & 55.48 \\
\hline 4.00 & 58.17 & 88.48 & 92.06 & 92.15 & 92.15 & 92.15 & 92.14 & 91.83 & 75.95 & 56.37 \\
\hline 5.00 & 58.45 & 88.68 & 92.26 & 92.35 & 92.36 & 92.36 & 92.34 & 92.02 & 76.16 & 56.63 \\
\hline 6.00 & 59.56 & 94.70 & 98.74 & 98.84 & 98.85 & 98.85 & 98.83 & 98.49 & 80.37 & 57.40 \\
\hline 7.00 & 59.86 & 94.92 & 98.97 & 99.07 & 99.08 & 99.08 & 99.06 & 98.70 & 80.61 & 57.68 \\
\hline 8.00 & 60.77 & 99.40 & 104.09 & 104.22 & 104.23 & 104.23 & 104.20 & 103.77 & 83.79 & 58.33 \\
\hline 9.00 & 61.02 & 99.60 & 104.32 & 104.45 & 104.47 & 104.47 & 104.43 & 103.99 & 84.02 & 58.63 \\
\hline 10.00 & 61.89 & 103.92 & 108.99 & 109.14 & 109.16 & 109.16 & 109.11 & 108.65 & 86.92 & 59.25 \\
\hline 11.00 & 62.19 & 104.18 & 109.26 & 109.41 & 109.44 & 109.44 & 109.39 & 108.91 & 87.16 & 59.52 \\
\hline 12.00 & 62.83 & 106.91 & 112.21 & 112.37 & 112.39 & 112.39 & 112.34 & 111.84 & 89.02 & 60.00 \\
\hline 13.00 & 63.09 & 107.15 & 112.45 & 112.62 & 112.65 & 112.65 & 112.59 & 112.08 & 89.25 & 60.26 \\
\hline 14.00 & 63.69 & 109.78 & 115.29 & 115.46 & 115.50 & 115.49 & 115.43 & 114.91 & 91.04 & 60.72 \\
\hline 15.00 & 63.96 & 110.02 & 115.54 & 115.72 & 115.76 & 115.76 & 115.68 & 115.15 & 91.28 & 60.96 \\
\hline 16.00 & 64.40 & 111.32 & 116.94 & 117.12 & 117.16 & 117.16 & 117.08 & 116.54 & 92.20 & 61.30 \\
\hline 17.00 & 64.65 & 111.57 & 117.18 & 117.38 & 117.42 & 117.42 & 117.33 & 116.78 & 92.45 & 61.60 \\
\hline 18.00 & 65.04 & 112.90 & 118.61 & 118.81 & 118.85 & 118.85 & 118.76 & 118.20 & 93.39 & 61.94 \\
\hline 19.00 & 65.31 & 113.15 & 118.87 & 119.07 & 119.12 & 119.12 & 119.02 & 118.45 & 93.63 & 62.17 \\
\hline 20.00 & 65.76 & 114.75 & 120.58 & 120.79 & 120.85 & 120.84 & 120.74 & 120.16 & 94.75 & 62.50 \\
\hline 21.00 & 65.99 & 114.98 & 120.81 & 121.03 & 121.08 & 121.08 & 120.97 & 120.38 & 94.96 & 62.71 \\
\hline 22.00 & 59.77 & 64.92 & 66.67 & 66.86 & 66.92 & 66.92 & 66.79 & 66.27 & 62.34 & 58.90 \\
\hline 22.50 & 57.78 & 57.88 & 58.19 & 58.34 & 58.40 & 58.40 & 58.26 & 58.03 & 57.74 & 57.47 \\
\hline
\end{tabular}


Table 27. Temperature $\left({ }^{\circ} \mathrm{C}\right)$ map of the west side of plate 2TT at EOC 141A (32.4 EFPD).

\begin{tabular}{|c||c|c|c|c|c|c|c|c|c|c|}
\hline \multirow{2}{*}{$\begin{array}{c}\text { Length of } \\
\text { Plate (in) }\end{array}$} & \multicolumn{10}{|c|}{ Width of Plate (inches) } \\
\hline & 0.05 & 0.25 & 0.50 & 0.75 & 1.00 & 1.25 & 1.50 & 1.75 & 2.00 & 2.24 \\
\hline 0.125 & 51.96 & 51.77 & 51.76 & 51.76 & 51.76 & 51.76 & 51.76 & 51.77 & 51.80 & 51.95 \\
\hline 1.00 & 53.03 & 52.47 & 52.65 & 52.68 & 52.68 & 52.68 & 52.67 & 52.59 & 52.40 & 52.86 \\
\hline 2.00 & 56.99 & 81.40 & 84.41 & 84.48 & 84.48 & 84.48 & 84.47 & 84.21 & 70.98 & 55.32 \\
\hline 3.00 & 57.37 & 81.68 & 84.71 & 84.79 & 84.79 & 84.79 & 84.78 & 84.50 & 71.28 & 55.66 \\
\hline 4.00 & 58.57 & 88.48 & 92.06 & 92.14 & 92.15 & 92.15 & 92.13 & 91.83 & 75.94 & 56.48 \\
\hline 5.00 & 58.86 & 88.68 & 92.26 & 92.35 & 92.35 & 92.35 & 92.34 & 92.02 & 76.15 & 56.74 \\
\hline 6.00 & 60.03 & 94.70 & 98.74 & 98.84 & 98.84 & 98.84 & 98.83 & 98.49 & 80.36 & 57.52 \\
\hline 7.00 & 60.34 & 94.92 & 98.96 & 99.06 & 99.07 & 99.07 & 99.05 & 98.70 & 80.60 & 57.79 \\
\hline 8.00 & 61.29 & 99.41 & 104.08 & 104.20 & 104.22 & 104.22 & 104.19 & 103.77 & 83.79 & 58.45 \\
\hline 9.00 & 61.56 & 99.61 & 104.31 & 104.44 & 104.46 & 104.45 & 104.42 & 103.99 & 84.01 & 58.81 \\
\hline 10.00 & 62.47 & 103.93 & 108.98 & 109.12 & 109.14 & 109.14 & 109.10 & 108.65 & 86.91 & 59.43 \\
\hline 11.00 & 62.78 & 104.20 & 109.26 & 109.40 & 109.42 & 109.42 & 109.37 & 108.91 & 87.16 & 59.68 \\
\hline 12.00 & 63.44 & 106.92 & 112.20 & 112.35 & 112.37 & 112.37 & 112.32 & 111.84 & 89.02 & 60.16 \\
\hline 13.00 & 63.71 & 107.16 & 112.44 & 112.60 & 112.62 & 112.62 & 112.57 & 112.08 & 89.26 & 60.43 \\
\hline 14.00 & 64.34 & 109.80 & 115.28 & 115.44 & 115.47 & 115.46 & 115.41 & 114.91 & 91.05 & 60.90 \\
\hline 15.00 & 64.62 & 110.05 & 115.53 & 115.70 & 115.73 & 115.72 & 115.67 & 115.16 & 91.28 & 61.13 \\
\hline 16.00 & 65.06 & 111.35 & 116.92 & 117.10 & 117.13 & 117.12 & 117.06 & 116.54 & 92.21 & 61.45 \\
\hline 17.00 & 65.32 & 111.59 & 117.17 & 117.35 & 117.38 & 117.38 & 117.31 & 116.78 & 92.46 & 61.80 \\
\hline 18.00 & 65.73 & 112.93 & 118.59 & 118.78 & 118.81 & 118.81 & 118.74 & 118.20 & 93.40 & 62.16 \\
\hline 19.00 & 65.99 & 113.18 & 118.85 & 119.04 & 119.08 & 119.07 & 119.00 & 118.45 & 93.64 & 62.38 \\
\hline 20.00 & 66.45 & 114.78 & 120.56 & 120.76 & 120.80 & 120.79 & 120.71 & 120.16 & 94.76 & 62.68 \\
\hline 21.00 & 66.68 & 115.01 & 120.79 & 120.99 & 121.03 & 121.03 & 120.94 & 120.38 & 94.98 & 62.88 \\
\hline 22.00 & 60.15 & 64.95 & 66.66 & 66.83 & 66.88 & 66.87 & 66.76 & 66.28 & 62.38 & 59.02 \\
\hline 22.50 & 58.11 & 57.91 & 58.17 & 58.30 & 58.36 & 58.35 & 58.24 & 58.03 & 57.78 & 57.66 \\
\hline
\end{tabular}


Table 28. Temperature $\left({ }^{\circ} \mathrm{C}\right)$ map of the east side of plate 2BZ at EOC 141A (32.4 EFPD).

\begin{tabular}{|c||c|c|c|c|c|c|c|c|c|c|}
\hline \multirow{2}{*}{$\begin{array}{c}\text { Length of } \\
\text { Plate (in) }\end{array}$} & 0.05 & 0.25 & 0.50 & 0.75 & 1.00 & 1.25 & 1.50 & 1.75 & 2.00 & 2.24 \\
\hline 22.625 & 57.91 & 57.92 & 58.24 & 58.39 & 58.46 & 58.45 & 58.32 & 58.08 & 57.78 & 57.60 \\
\hline 23.00 & 59.43 & 63.43 & 64.86 & 65.05 & 65.11 & 65.11 & 64.97 & 64.52 & 61.38 & 58.67 \\
\hline 24.00 & 64.60 & 103.66 & 108.48 & 108.70 & 108.76 & 108.76 & 108.63 & 108.10 & 87.70 & 61.94 \\
\hline 25.00 & 64.78 & 103.85 & 108.66 & 108.89 & 108.95 & 108.95 & 108.82 & 108.28 & 87.90 & 62.11 \\
\hline 26.00 & 64.82 & 103.11 & 107.85 & 108.08 & 108.15 & 108.15 & 108.01 & 107.47 & 87.49 & 62.23 \\
\hline 27.00 & 64.98 & 103.31 & 108.06 & 108.29 & 108.36 & 108.36 & 108.22 & 107.67 & 87.68 & 62.39 \\
\hline 28.00 & 65.12 & 103.35 & 108.09 & 108.32 & 108.39 & 108.39 & 108.25 & 107.70 & 87.76 & 62.51 \\
\hline 29.00 & 65.26 & 103.55 & 108.28 & 108.52 & 108.59 & 108.59 & 108.44 & 107.89 & 87.93 & 62.61 \\
\hline 30.00 & 65.34 & 103.25 & 107.94 & 108.18 & 108.26 & 108.25 & 108.10 & 107.55 & 87.80 & 62.71 \\
\hline 31.00 & 65.46 & 103.43 & 108.12 & 108.37 & 108.45 & 108.44 & 108.29 & 107.73 & 87.97 & 62.83 \\
\hline 32.00 & 65.40 & 102.32 & 106.90 & 107.15 & 107.23 & 107.23 & 107.07 & 106.52 & 87.30 & 62.83 \\
\hline 33.00 & 65.51 & 102.51 & 107.09 & 107.35 & 107.43 & 107.42 & 107.26 & 106.71 & 87.48 & 62.91 \\
\hline 34.00 & 65.30 & 100.45 & 104.84 & 105.10 & 105.18 & 105.18 & 105.01 & 104.46 & 86.19 & 62.80 \\
\hline 35.00 & 65.38 & 100.61 & 105.01 & 105.26 & 105.35 & 105.34 & 105.17 & 104.62 & 86.34 & 62.91 \\
\hline 36.00 & 65.15 & 98.59 & 102.79 & 103.05 & 103.13 & 103.13 & 102.96 & 102.41 & 85.08 & 62.79 \\
\hline 37.00 & 65.22 & 98.74 & 102.94 & 103.20 & 103.28 & 103.28 & 103.11 & 102.56 & 85.21 & 62.84 \\
\hline 38.00 & 64.85 & 95.85 & 99.77 & 100.03 & 100.12 & 100.11 & 99.94 & 99.41 & 83.37 & 62.59 \\
\hline 39.00 & 64.88 & 95.97 & 99.89 & 100.16 & 100.25 & 100.24 & 100.06 & 99.53 & 83.48 & 62.62 \\
\hline 40.00 & 64.36 & 92.10 & 95.65 & 95.91 & 96.01 & 96.00 & 95.82 & 95.31 & 81.01 & 62.30 \\
\hline 41.00 & 64.36 & 92.20 & 95.75 & 96.01 & 96.10 & 96.09 & 95.91 & 95.40 & 81.09 & 62.30 \\
\hline 42.00 & 63.66 & 87.41 & 90.36 & 90.59 & 90.68 & 90.68 & 90.50 & 90.06 & 77.93 & 61.84 \\
\hline 43.00 & 63.63 & 87.47 & 90.41 & 90.65 & 90.74 & 90.73 & 90.56 & 90.12 & 77.98 & 61.80 \\
\hline 44.00 & 60.47 & 62.42 & 62.99 & 63.22 & 63.32 & 63.31 & 63.11 & 62.77 & 61.92 & 59.81 \\
\hline 45.00 & 59.92 & 61.87 & 62.27 & 62.48 & 62.59 & 62.58 & 62.38 & 62.11 & 61.50 & 59.36 \\
\hline
\end{tabular}


Table 29. Temperature $\left({ }^{\circ} \mathrm{C}\right)$ map of the west side of plate $2 \mathrm{BZ}$ at EOC 141A (32.4 EFPD).

\begin{tabular}{|c||c|c|c|c|c|c|c|c|c|c|}
\hline \multirow{2}{*}{$\begin{array}{c}\text { Length of } \\
\text { Plate (in) }\end{array}$} & \multicolumn{10}{|c|}{ Width of Plate (inches) } \\
\hline 22.625 & 58.20 & 57.95 & 58.23 & 58.36 & 58.41 & 58.41 & 58.30 & 58.08 & 57.82 & 57.74 \\
\hline 23.00 & 59.78 & 63.46 & 64.85 & 65.01 & 65.07 & 65.06 & 64.95 & 64.53 & 61.42 & 58.79 \\
\hline 24.00 & 65.24 & 103.73 & 108.50 & 108.69 & 108.74 & 108.74 & 108.64 & 108.14 & 87.76 & 62.11 \\
\hline 25.00 & 65.38 & 103.89 & 108.64 & 108.84 & 108.89 & 108.88 & 108.78 & 108.28 & 87.92 & 62.27 \\
\hline 26.00 & 65.41 & 103.15 & 107.83 & 108.03 & 108.08 & 108.08 & 107.97 & 107.47 & 87.51 & 62.42 \\
\hline 27.00 & 65.56 & 103.35 & 108.04 & 108.24 & 108.29 & 108.29 & 108.18 & 107.67 & 87.71 & 62.58 \\
\hline 28.00 & 65.69 & 103.39 & 108.07 & 108.27 & 108.32 & 108.32 & 108.21 & 107.70 & 87.79 & 62.68 \\
\hline 29.00 & 65.83 & 103.60 & 108.28 & 108.48 & 108.54 & 108.53 & 108.42 & 107.91 & 87.98 & 62.76 \\
\hline 30.00 & 65.89 & 103.28 & 107.92 & 108.12 & 108.18 & 108.18 & 108.06 & 107.55 & 87.83 & 62.83 \\
\hline 31.00 & 66.00 & 103.46 & 108.10 & 108.31 & 108.37 & 108.36 & 108.24 & 107.73 & 88.00 & 62.96 \\
\hline 32.00 & 65.92 & 102.36 & 106.88 & 107.09 & 107.15 & 107.14 & 107.02 & 106.51 & 87.33 & 62.95 \\
\hline 33.00 & 66.02 & 102.55 & 107.07 & 107.28 & 107.34 & 107.34 & 107.21 & 106.70 & 87.50 & 63.01 \\
\hline 34.00 & 65.77 & 100.49 & 104.82 & 105.03 & 105.09 & 105.09 & 104.96 & 104.46 & 86.22 & 62.90 \\
\hline 35.00 & 65.85 & 100.65 & 104.98 & 105.19 & 105.26 & 105.25 & 105.12 & 104.62 & 86.37 & 63.03 \\
\hline 36.00 & 65.58 & 98.63 & 102.76 & 102.98 & 103.04 & 103.03 & 102.90 & 102.41 & 85.11 & 62.91 \\
\hline 37.00 & 65.64 & 98.78 & 102.91 & 103.13 & 103.19 & 103.18 & 103.05 & 102.56 & 85.24 & 62.94 \\
\hline 38.00 & 65.22 & 95.88 & 99.74 & 99.96 & 100.02 & 100.02 & 99.88 & 99.40 & 83.40 & 62.65 \\
\hline 39.00 & 65.25 & 96.01 & 99.87 & 100.08 & 100.15 & 100.14 & 100.01 & 99.53 & 83.52 & 62.67 \\
\hline 40.00 & 64.68 & 92.14 & 95.63 & 95.84 & 95.91 & 95.90 & 95.76 & 95.30 & 81.04 & 62.35 \\
\hline 41.00 & 64.66 & 92.23 & 95.72 & 95.93 & 96.00 & 95.99 & 95.85 & 95.40 & 81.13 & 62.33 \\
\hline 42.00 & 63.91 & 87.44 & 90.33 & 90.52 & 90.59 & 90.58 & 90.45 & 90.06 & 77.96 & 61.85 \\
\hline 43.00 & 63.85 & 87.50 & 90.39 & 90.57 & 90.64 & 90.63 & 90.50 & 90.11 & 78.01 & 61.81 \\
\hline 44.00 & 60.51 & 62.44 & 62.97 & 63.16 & 63.24 & 63.23 & 63.07 & 62.76 & 61.97 & 59.79 \\
\hline 45.00 & 59.93 & 61.90 & 62.25 & 62.42 & 62.51 & 62.49 & 62.34 & 62.10 & 61.54 & 59.34 \\
\hline
\end{tabular}


Table 30. Temperature $\left({ }^{\circ} \mathrm{C}\right)$ map of the east side of plate 2TT at EOC 142A (80.4 EFPD).

\begin{tabular}{|c||c|c|c|c|c|c|c|c|c|c|}
\hline \multirow{2}{*}{$\begin{array}{c}\text { Length of } \\
\text { Plate (in) }\end{array}$} & \multicolumn{10}{|c|}{ Width of Plate (inches) } \\
\hline 0.125 & 51.93 & 51.78 & 51.77 & 51.77 & 51.77 & 51.77 & 51.77 & 51.77 & 51.80 & 51.94 \\
\hline 1.00 & 52.94 & 52.48 & 52.66 & 52.68 & 52.69 & 52.69 & 52.68 & 52.60 & 52.41 & 52.83 \\
\hline 2.00 & 56.72 & 81.50 & 84.51 & 84.58 & 84.58 & 84.58 & 84.57 & 84.31 & 71.06 & 55.28 \\
\hline 3.00 & 57.06 & 81.75 & 84.79 & 84.87 & 84.87 & 84.87 & 84.86 & 84.58 & 71.34 & 55.60 \\
\hline 4.00 & 57.98 & 86.98 & 90.43 & 90.51 & 90.52 & 90.52 & 90.51 & 90.20 & 74.93 & 56.26 \\
\hline 5.00 & 58.24 & 87.15 & 90.61 & 90.70 & 90.71 & 90.71 & 90.69 & 90.38 & 75.12 & 56.51 \\
\hline 6.00 & 59.06 & 91.25 & 95.03 & 95.13 & 95.13 & 95.13 & 95.11 & 94.78 & 77.99 & 57.09 \\
\hline 7.00 & 59.34 & 91.45 & 95.23 & 95.33 & 95.34 & 95.34 & 95.31 & 94.98 & 78.20 & 57.34 \\
\hline 8.00 & 59.94 & 94.02 & 97.99 & 98.09 & 98.11 & 98.11 & 98.08 & 97.73 & 80.04 & 57.80 \\
\hline 9.00 & 60.18 & 94.19 & 98.16 & 98.27 & 98.28 & 98.28 & 98.25 & 97.89 & 80.25 & 58.08 \\
\hline 10.00 & 60.69 & 96.15 & 100.33 & 100.45 & 100.47 & 100.47 & 100.43 & 100.03 & 81.67 & 58.48 \\
\hline 11.00 & 60.96 & 96.34 & 100.55 & 100.69 & 100.71 & 100.71 & 100.66 & 100.25 & 81.88 & 58.71 \\
\hline 12.00 & 61.30 & 97.21 & 101.55 & 101.69 & 101.72 & 101.72 & 101.67 & 101.24 & 82.55 & 59.00 \\
\hline 13.00 & 61.53 & 97.38 & 101.75 & 101.90 & 101.93 & 101.93 & 101.87 & 101.43 & 82.75 & 59.23 \\
\hline 14.00 & 61.81 & 97.96 & 102.42 & 102.57 & 102.60 & 102.60 & 102.54 & 102.09 & 83.22 & 59.49 \\
\hline 15.00 & 62.04 & 98.12 & 102.62 & 102.78 & 102.81 & 102.81 & 102.74 & 102.29 & 83.41 & 59.69 \\
\hline 16.00 & 62.32 & 98.63 & 103.16 & 103.32 & 103.36 & 103.35 & 103.28 & 102.82 & 83.81 & 59.92 \\
\hline 17.00 & 62.54 & 98.83 & 103.35 & 103.52 & 103.56 & 103.56 & 103.48 & 103.01 & 84.01 & 60.19 \\
\hline 18.00 & 62.84 & 99.74 & 104.34 & 104.51 & 104.55 & 104.55 & 104.47 & 103.99 & 84.67 & 60.47 \\
\hline 19.00 & 63.06 & 99.95 & 104.54 & 104.72 & 104.76 & 104.76 & 104.68 & 104.19 & 84.87 & 60.66 \\
\hline 20.00 & 63.17 & 99.33 & 103.85 & 104.04 & 104.08 & 104.08 & 103.99 & 103.50 & 84.53 & 60.77 \\
\hline 21.00 & 63.32 & 99.48 & 104.00 & 104.19 & 104.24 & 104.24 & 104.14 & 103.65 & 84.68 & 60.92 \\
\hline 22.00 & 58.77 & 62.08 & 63.38 & 63.53 & 63.59 & 63.58 & 63.47 & 63.08 & 60.23 & 58.13 \\
\hline 22.50 & 57.31 & 56.88 & 57.08 & 57.20 & 57.26 & 57.25 & 57.14 & 56.97 & 56.85 & 57.10 \\
\hline
\end{tabular}


Table 31. Temperature $\left({ }^{\circ} \mathrm{C}\right)$ map of the west side of plate 2TT at EOC 142A (80.4 EFPD).

\begin{tabular}{|c||c|c|c|c|c|c|c|c|c|c|}
\hline \multirow{2}{*}{$\begin{array}{c}\text { Length of } \\
\text { Plate (in) }\end{array}$} & \multicolumn{10}{|c|}{ Width of Plate (inches) } \\
\hline 0.125 & 51.97 & 51.78 & 51.77 & 51.77 & 51.77 & 51.77 & 51.77 & 51.77 & 51.80 & 51.97 \\
\hline 1.00 & 53.07 & 52.49 & 52.66 & 52.68 & 52.69 & 52.69 & 52.68 & 52.60 & 52.41 & 52.90 \\
\hline 2.00 & 57.05 & 81.49 & 84.51 & 84.58 & 84.58 & 84.58 & 84.57 & 84.31 & 71.05 & 55.37 \\
\hline 3.00 & 57.41 & 81.76 & 84.79 & 84.86 & 84.87 & 84.87 & 84.86 & 84.58 & 71.33 & 55.70 \\
\hline 4.00 & 58.38 & 86.98 & 90.43 & 90.51 & 90.52 & 90.52 & 90.50 & 90.20 & 74.92 & 56.38 \\
\hline 5.00 & 58.66 & 87.16 & 90.61 & 90.70 & 90.70 & 90.70 & 90.69 & 90.38 & 75.11 & 56.63 \\
\hline 6.00 & 59.51 & 91.26 & 95.03 & 95.12 & 95.13 & 95.13 & 95.11 & 94.78 & 77.98 & 57.21 \\
\hline 7.00 & 59.80 & 91.45 & 95.22 & 95.32 & 95.33 & 95.33 & 95.31 & 94.97 & 78.20 & 57.46 \\
\hline 8.00 & 60.44 & 94.03 & 97.98 & 98.08 & 98.09 & 98.09 & 98.07 & 97.73 & 80.04 & 57.92 \\
\hline 9.00 & 60.69 & 94.20 & 98.15 & 98.25 & 98.27 & 98.27 & 98.24 & 97.89 & 80.24 & 58.27 \\
\hline 10.00 & 61.23 & 96.15 & 100.32 & 100.44 & 100.45 & 100.45 & 100.42 & 100.02 & 81.67 & 58.66 \\
\hline 11.00 & 61.51 & 96.35 & 100.54 & 100.67 & 100.69 & 100.68 & 100.65 & 100.25 & 81.88 & 58.87 \\
\hline 12.00 & 61.86 & 97.22 & 101.54 & 101.67 & 101.69 & 101.69 & 101.65 & 101.24 & 82.55 & 59.16 \\
\hline 13.00 & 62.10 & 97.39 & 101.74 & 101.88 & 101.90 & 101.90 & 101.85 & 101.43 & 82.75 & 59.40 \\
\hline 14.00 & 62.40 & 97.97 & 102.41 & 102.55 & 102.57 & 102.57 & 102.52 & 102.09 & 83.22 & 59.67 \\
\hline 15.00 & 62.63 & 98.14 & 102.61 & 102.75 & 102.78 & 102.77 & 102.72 & 102.28 & 83.41 & 59.85 \\
\hline 16.00 & 62.92 & 98.65 & 103.14 & 103.29 & 103.32 & 103.32 & 103.26 & 102.82 & 83.81 & 60.07 \\
\hline 17.00 & 63.14 & 98.85 & 103.34 & 103.49 & 103.52 & 103.52 & 103.46 & 103.01 & 84.02 & 60.39 \\
\hline 18.00 & 63.45 & 99.76 & 104.32 & 104.48 & 104.51 & 104.50 & 104.44 & 103.99 & 84.68 & 60.69 \\
\hline 19.00 & 63.68 & 99.97 & 104.53 & 104.69 & 104.72 & 104.71 & 104.65 & 104.19 & 84.88 & 60.88 \\
\hline 20.00 & 63.78 & 99.35 & 103.83 & 104.00 & 104.03 & 104.03 & 103.96 & 103.50 & 84.54 & 60.95 \\
\hline 21.00 & 63.94 & 99.51 & 103.98 & 104.15 & 104.19 & 104.18 & 104.11 & 103.65 & 84.69 & 61.09 \\
\hline 22.00 & 59.14 & 62.10 & 63.36 & 63.50 & 63.54 & 63.54 & 63.45 & 63.07 & 60.26 & 58.27 \\
\hline 22.50 & 57.66 & 56.90 & 57.07 & 57.17 & 57.21 & 57.20 & 57.12 & 56.97 & 56.88 & 57.30 \\
\hline
\end{tabular}


Table 32. Temperature $\left({ }^{\circ} \mathrm{C}\right)$ map of the east side of plate 2BZ at EOC 142A (80.4 EFPD).

\begin{tabular}{|c||c|c|c|c|c|c|c|c|c|c|}
\hline \multirow{2}{*}{$\begin{array}{l}\text { Length of } \\
\text { Plate (in) }\end{array}$} & \multicolumn{10}{|c|}{ Width of Plate (inches) } \\
\hline 22.625 & 57.41 & 56.92 & 57.12 & 57.24 & 57.30 & 57.29 & 57.18 & 57.01 & 56.88 & 57.20 \\
\hline 23.00 & 58.54 & 61.00 & 62.05 & 62.20 & 62.26 & 62.25 & 62.14 & 61.80 & 59.54 & 57.99 \\
\hline 24.00 & 62.37 & 91.28 & 94.81 & 94.97 & 95.01 & 95.01 & 94.92 & 94.53 & 79.25 & 60.42 \\
\hline 25.00 & 62.52 & 91.43 & 94.95 & 95.11 & 95.16 & 95.16 & 95.06 & 94.67 & 79.42 & 60.57 \\
\hline 26.00 & 62.59 & 91.11 & 94.59 & 94.76 & 94.81 & 94.81 & 94.71 & 94.31 & 79.27 & 60.69 \\
\hline 27.00 & 62.71 & 91.24 & 94.73 & 94.90 & 94.95 & 94.95 & 94.84 & 94.45 & 79.42 & 60.82 \\
\hline 28.00 & 62.78 & 91.03 & 94.49 & 94.66 & 94.71 & 94.71 & 94.61 & 94.21 & 79.32 & 60.89 \\
\hline 29.00 & 62.91 & 91.18 & 94.64 & 94.81 & 94.87 & 94.86 & 94.76 & 94.36 & 79.47 & 60.98 \\
\hline 30.00 & 63.03 & 91.42 & 94.88 & 95.08 & 95.14 & 95.14 & 95.01 & 94.61 & 79.68 & 61.09 \\
\hline 31.00 & 63.12 & 91.55 & 95.03 & 95.23 & 95.29 & 95.29 & 95.16 & 94.73 & 79.82 & 61.19 \\
\hline 32.00 & 63.14 & 91.20 & 94.63 & 94.83 & 94.89 & 94.89 & 94.76 & 94.34 & 79.63 & 61.23 \\
\hline 33.00 & 63.23 & 91.34 & 94.78 & 94.99 & 95.06 & 95.05 & 94.92 & 94.48 & 79.76 & 61.30 \\
\hline 34.00 & 63.22 & 90.91 & 94.29 & 94.50 & 94.57 & 94.56 & 94.43 & 94.01 & 79.52 & 61.32 \\
\hline 35.00 & 63.28 & 91.03 & 94.43 & 94.64 & 94.71 & 94.71 & 94.57 & 94.13 & 79.64 & 61.40 \\
\hline 36.00 & 63.28 & 90.69 & 94.05 & 94.26 & 94.33 & 94.32 & 94.18 & 93.76 & 79.46 & 61.42 \\
\hline 37.00 & 63.33 & 90.82 & 94.20 & 94.41 & 94.48 & 94.47 & 94.33 & 93.89 & 79.58 & 61.46 \\
\hline 38.00 & 63.14 & 89.39 & 92.61 & 92.81 & 92.87 & 92.87 & 92.74 & 92.33 & 78.65 & 61.33 \\
\hline 39.00 & 63.17 & 89.50 & 92.72 & 92.91 & 92.97 & 92.97 & 92.84 & 92.44 & 78.75 & 61.35 \\
\hline 40.00 & 62.93 & 87.76 & 90.83 & 91.03 & 91.09 & 91.09 & 90.96 & 90.56 & 77.61 & 61.21 \\
\hline 41.00 & 62.93 & 87.85 & 90.92 & 91.12 & 91.18 & 91.18 & 91.05 & 90.65 & 77.70 & 61.20 \\
\hline 42.00 & 62.49 & 84.92 & 87.74 & 87.93 & 88.00 & 88.00 & 87.86 & 87.47 & 75.76 & 60.91 \\
\hline 43.00 & 62.46 & 84.99 & 87.80 & 88.00 & 88.06 & 88.06 & 87.93 & 87.54 & 75.82 & 60.87 \\
\hline 44.00 & 59.38 & 60.59 & 61.05 & 61.23 & 61.31 & 61.31 & 61.15 & 60.87 & 60.22 & 58.93 \\
\hline 45.00 & 58.84 & 60.05 & 60.34 & 60.51 & 60.59 & 60.58 & 60.43 & 60.21 & 59.79 & 58.49 \\
\hline
\end{tabular}


Table 33. Temperature $\left({ }^{\circ} \mathrm{C}\right)$ map of the west side of plate $2 \mathrm{BZ}$ at EOC 142A (80.4 EFPD).

\begin{tabular}{|c||c|c|c|c|c|c|c|c|c|c|}
\hline \multirow{2}{*}{$\begin{array}{c}\text { Length of } \\
\text { Plate (in) }\end{array}$} & \multicolumn{10}{|c|}{ Width of Plate (inches) } \\
\hline 22.625 & 57.73 & 56.94 & 57.11 & 57.21 & 57.25 & 57.25 & 57.16 & 57.00 & 56.91 & 57.36 \\
\hline 23.00 & 58.90 & 61.02 & 62.04 & 62.17 & 62.21 & 62.20 & 62.11 & 61.79 & 59.56 & 58.12 \\
\hline 24.00 & 62.92 & 91.30 & 94.78 & 94.92 & 94.96 & 94.95 & 94.88 & 94.52 & 79.27 & 60.57 \\
\hline 25.00 & 63.06 & 91.45 & 94.93 & 95.07 & 95.10 & 95.10 & 95.03 & 94.66 & 79.44 & 60.73 \\
\hline 26.00 & 63.13 & 91.12 & 94.57 & 94.71 & 94.75 & 94.74 & 94.67 & 94.30 & 79.28 & 60.89 \\
\hline 27.00 & 63.24 & 91.26 & 94.70 & 94.85 & 94.88 & 94.88 & 94.80 & 94.44 & 79.43 & 61.02 \\
\hline 28.00 & 63.30 & 91.05 & 94.46 & 94.61 & 94.64 & 94.64 & 94.56 & 94.20 & 79.33 & 61.07 \\
\hline 29.00 & 63.42 & 91.20 & 94.61 & 94.76 & 94.79 & 94.79 & 94.71 & 94.35 & 79.48 & 61.13 \\
\hline 30.00 & 63.53 & 91.44 & 94.86 & 95.01 & 95.06 & 95.05 & 94.96 & 94.59 & 79.69 & 61.23 \\
\hline 31.00 & 63.62 & 91.57 & 94.99 & 95.16 & 95.20 & 95.20 & 95.11 & 94.72 & 79.83 & 61.33 \\
\hline 32.00 & 63.62 & 91.21 & 94.59 & 94.76 & 94.80 & 94.80 & 94.70 & 94.33 & 79.64 & 61.36 \\
\hline 33.00 & 63.70 & 91.35 & 94.75 & 94.91 & 94.96 & 94.96 & 94.86 & 94.47 & 79.78 & 61.41 \\
\hline 34.00 & 63.67 & 90.92 & 94.26 & 94.42 & 94.47 & 94.47 & 94.37 & 93.99 & 79.53 & 61.43 \\
\hline 35.00 & 63.72 & 91.04 & 94.39 & 94.56 & 94.61 & 94.60 & 94.51 & 94.11 & 79.65 & 61.54 \\
\hline 36.00 & 63.70 & 90.71 & 94.01 & 94.18 & 94.23 & 94.22 & 94.12 & 93.74 & 79.47 & 61.56 \\
\hline 37.00 & 63.73 & 90.83 & 94.15 & 94.33 & 94.37 & 94.37 & 94.27 & 93.87 & 79.59 & 61.58 \\
\hline 38.00 & 63.52 & 89.40 & 92.57 & 92.73 & 92.77 & 92.77 & 92.68 & 92.31 & 78.65 & 61.41 \\
\hline 39.00 & 63.53 & 89.50 & 92.68 & 92.83 & 92.88 & 92.87 & 92.78 & 92.41 & 78.75 & 61.41 \\
\hline 40.00 & 63.26 & 87.77 & 90.79 & 90.95 & 90.99 & 90.99 & 90.89 & 90.53 & 77.62 & 61.27 \\
\hline 41.00 & 63.24 & 87.86 & 90.88 & 91.03 & 91.08 & 91.08 & 90.98 & 90.62 & 77.70 & 61.26 \\
\hline 42.00 & 62.76 & 84.93 & 87.69 & 87.85 & 87.90 & 87.89 & 87.79 & 87.44 & 75.77 & 60.95 \\
\hline 43.00 & 62.70 & 84.99 & 87.76 & 87.91 & 87.96 & 87.95 & 87.86 & 87.51 & 75.83 & 60.90 \\
\hline 44.00 & 59.45 & 60.59 & 61.01 & 61.16 & 61.22 & 61.21 & 61.09 & 60.84 & 60.23 & 58.93 \\
\hline 45.00 & 58.89 & 60.05 & 60.30 & 60.44 & 60.50 & 60.49 & 60.37 & 60.19 & 59.81 & 58.49 \\
\hline
\end{tabular}


Table 34. Temperature $\left({ }^{\circ} \mathrm{C}\right)$ map of the east side of plate 2TT at EOC 142B (132.4 EFPD).

\begin{tabular}{|c||c|c|c|c|c|c|c|c|c|c|}
\hline \multirow{2}{*}{$\begin{array}{c}\text { Length of } \\
\text { Plate (in) }\end{array}$} & \multicolumn{10}{|c|}{ Width of Plate (inches) } \\
\hline & 0.05 & 0.25 & 0.50 & 0.75 & 1.00 & 1.25 & 1.50 & 1.75 & 2.00 & 2.24 \\
\hline 0.125 & 51.94 & 51.78 & 51.77 & 51.77 & 51.77 & 51.77 & 51.77 & 51.77 & 51.81 & 51.95 \\
\hline 1.00 & 52.95 & 52.39 & 52.53 & 52.54 & 52.55 & 52.55 & 52.54 & 52.48 & 52.37 & 52.87 \\
\hline 2.00 & 56.41 & 76.93 & 79.51 & 79.58 & 79.58 & 79.58 & 79.57 & 79.34 & 68.11 & 55.25 \\
\hline 3.00 & 56.74 & 77.16 & 79.76 & 79.82 & 79.83 & 79.83 & 79.82 & 79.57 & 68.36 & 55.57 \\
\hline 4.00 & 57.45 & 80.42 & 83.28 & 83.36 & 83.36 & 83.36 & 83.35 & 83.08 & 70.61 & 56.14 \\
\hline 5.00 & 57.71 & 80.58 & 83.45 & 83.52 & 83.53 & 83.53 & 83.51 & 83.24 & 70.80 & 56.40 \\
\hline 6.00 & 58.35 & 83.09 & 86.16 & 86.24 & 86.25 & 86.25 & 86.23 & 85.94 & 72.57 & 56.90 \\
\hline 7.00 & 58.63 & 83.27 & 86.34 & 86.42 & 86.43 & 86.43 & 86.41 & 86.11 & 72.77 & 57.16 \\
\hline 8.00 & 59.06 & 84.43 & 87.58 & 87.67 & 87.68 & 87.68 & 87.66 & 87.35 & 73.64 & 57.54 \\
\hline 9.00 & 59.31 & 84.59 & 87.74 & 87.83 & 87.84 & 87.84 & 87.81 & 87.51 & 73.84 & 57.86 \\
\hline 10.00 & 59.67 & 85.32 & 88.51 & 88.61 & 88.63 & 88.63 & 88.59 & 88.28 & 74.41 & 58.17 \\
\hline 11.00 & 59.95 & 85.50 & 88.69 & 88.79 & 88.81 & 88.81 & 88.77 & 88.45 & 74.61 & 58.40 \\
\hline 12.00 & 60.25 & 85.97 & 89.18 & 89.29 & 89.31 & 89.30 & 89.26 & 88.94 & 75.01 & 58.69 \\
\hline 13.00 & 60.48 & 86.13 & 89.34 & 89.45 & 89.47 & 89.47 & 89.42 & 89.10 & 75.20 & 58.94 \\
\hline 14.00 & 60.69 & 86.02 & 89.20 & 89.31 & 89.34 & 89.34 & 89.29 & 88.96 & 75.20 & 59.17 \\
\hline 15.00 & 60.92 & 86.18 & 89.36 & 89.47 & 89.50 & 89.50 & 89.44 & 89.11 & 75.38 & 59.36 \\
\hline 16.00 & 61.16 & 86.33 & 89.49 & 89.62 & 89.64 & 89.64 & 89.59 & 89.25 & 75.55 & 59.58 \\
\hline 17.00 & 61.38 & 86.48 & 89.65 & 89.77 & 89.80 & 89.80 & 89.74 & 89.41 & 75.75 & 59.87 \\
\hline 18.00 & 61.58 & 86.49 & 89.64 & 89.76 & 89.80 & 89.79 & 89.73 & 89.39 & 75.84 & 60.11 \\
\hline 19.00 & 61.78 & 86.64 & 89.79 & 89.92 & 89.95 & 89.95 & 89.88 & 89.54 & 76.01 & 60.29 \\
\hline 20.00 & 62.01 & 86.74 & 89.87 & 90.01 & 90.04 & 90.04 & 89.97 & 89.62 & 76.14 & 60.46 \\
\hline 21.00 & 62.18 & 86.87 & 90.00 & 90.14 & 90.18 & 90.18 & 90.10 & 89.76 & 76.30 & 60.62 \\
\hline 22.00 & 58.63 & 60.20 & 61.07 & 61.21 & 61.25 & 61.25 & 61.15 & 60.86 & 59.00 & 58.26 \\
\hline 22.50 & 57.45 & 56.58 & 56.70 & 56.80 & 56.85 & 56.85 & 56.75 & 56.62 & 56.62 & 57.37 \\
\hline
\end{tabular}


Table 35. Temperature $\left({ }^{\circ} \mathrm{C}\right)$ map of the west side of plate 2TT at EOC 142B (132.4 EFPD).

\begin{tabular}{|c||c|c|c|c|c|c|c|c|c|c|}
\hline \multirow{2}{*}{$\begin{array}{c}\text { Length of } \\
\text { Plate (in) }\end{array}$} & \multicolumn{10}{|c|}{ Width of Plate (inches) } \\
\hline 0.125 & 51.98 & 51.78 & 51.77 & 51.77 & 51.77 & 51.77 & 51.77 & 51.77 & 51.81 & 51.98 \\
\hline 1.00 & 53.18 & 52.40 & 52.53 & 52.55 & 52.55 & 52.55 & 52.54 & 52.48 & 52.37 & 53.00 \\
\hline 2.00 & 57.26 & 76.95 & 79.53 & 79.60 & 79.60 & 79.60 & 79.59 & 79.35 & 68.11 & 55.68 \\
\hline 3.00 & 57.66 & 77.21 & 79.80 & 79.87 & 79.88 & 79.88 & 79.86 & 79.61 & 68.39 & 56.02 \\
\hline 4.00 & 58.52 & 80.50 & 83.36 & 83.44 & 83.45 & 83.45 & 83.43 & 83.16 & 70.67 & 56.69 \\
\hline 5.00 & 58.83 & 80.71 & 83.57 & 83.65 & 83.66 & 83.66 & 83.64 & 83.36 & 70.89 & 56.96 \\
\hline 6.00 & 59.62 & 83.26 & 86.32 & 86.41 & 86.42 & 86.42 & 86.40 & 86.11 & 72.70 & 57.53 \\
\hline 7.00 & 59.96 & 83.50 & 86.56 & 86.65 & 86.66 & 86.66 & 86.63 & 86.34 & 72.95 & 57.80 \\
\hline 8.00 & 60.52 & 84.72 & 87.86 & 87.95 & 87.97 & 87.97 & 87.94 & 87.63 & 73.87 & 58.25 \\
\hline 9.00 & 60.81 & 84.93 & 88.07 & 88.17 & 88.19 & 88.19 & 88.15 & 87.84 & 74.12 & 58.67 \\
\hline 10.00 & 61.28 & 85.73 & 88.91 & 89.02 & 89.04 & 89.04 & 88.99 & 88.68 & 74.75 & 59.03 \\
\hline 11.00 & 61.60 & 85.98 & 89.16 & 89.27 & 89.29 & 89.29 & 89.25 & 88.92 & 75.01 & 59.24 \\
\hline 12.00 & 61.99 & 86.51 & 89.72 & 89.83 & 89.86 & 89.86 & 89.81 & 89.48 & 75.47 & 59.56 \\
\hline 13.00 & 62.26 & 86.74 & 89.94 & 90.06 & 90.09 & 90.09 & 90.03 & 89.70 & 75.72 & 59.85 \\
\hline 14.00 & 62.54 & 86.70 & 89.88 & 90.01 & 90.04 & 90.04 & 89.97 & 89.64 & 75.79 & 60.11 \\
\hline 15.00 & 62.81 & 86.93 & 90.11 & 90.24 & 90.27 & 90.27 & 90.20 & 89.86 & 76.02 & 60.30 \\
\hline 16.00 & 63.11 & 87.16 & 90.33 & 90.47 & 90.50 & 90.50 & 90.43 & 90.08 & 76.28 & 60.53 \\
\hline 17.00 & 63.36 & 87.40 & 90.56 & 90.70 & 90.74 & 90.74 & 90.66 & 90.31 & 76.54 & 60.92 \\
\hline 18.00 & 63.62 & 87.47 & 90.63 & 90.77 & 90.81 & 90.81 & 90.73 & 90.37 & 76.69 & 61.22 \\
\hline 19.00 & 63.86 & 87.71 & 90.86 & 91.00 & 91.04 & 91.04 & 90.96 & 90.60 & 76.93 & 61.39 \\
\hline 20.00 & 64.14 & 87.89 & 91.03 & 91.18 & 91.22 & 91.22 & 91.13 & 90.77 & 77.14 & 61.53 \\
\hline 21.00 & 64.34 & 88.10 & 91.24 & 91.39 & 91.44 & 91.43 & 91.34 & 90.98 & 77.36 & 61.70 \\
\hline 22.00 & 59.96 & 61.29 & 62.18 & 62.32 & 62.38 & 62.38 & 62.26 & 61.95 & 60.06 & 58.91 \\
\hline 22.50 & 58.47 & 57.72 & 57.85 & 57.97 & 58.03 & 58.02 & 57.91 & 57.75 & 57.71 & 57.92 \\
\hline
\end{tabular}


Table 36. Temperature $\left({ }^{\circ} \mathrm{C}\right)$ map of the east side of plate 2BZ at EOC 142B (132.4 EFPD).

\begin{tabular}{|c||c|c|c|c|c|c|c|c|c|c|}
\hline \multirow{2}{*}{$\begin{array}{c}\text { Length of } \\
\text { Plate (in) }\end{array}$} & \multicolumn{10}{|c|}{ Width of Plate (inches) } \\
\hline 22.625 & 57.55 & 56.61 & 56.73 & 56.84 & 56.89 & 56.88 & 56.78 & 56.65 & 56.64 & 57.46 \\
\hline 23.00 & 58.47 & 59.35 & 60.03 & 60.16 & 60.21 & 60.20 & 60.10 & 59.85 & 58.47 & 58.18 \\
\hline 24.00 & 61.49 & 80.00 & 82.49 & 82.63 & 82.67 & 82.67 & 82.58 & 82.28 & 71.83 & 60.32 \\
\hline 25.00 & 61.68 & 80.23 & 82.73 & 82.87 & 82.91 & 82.91 & 82.82 & 82.51 & 72.06 & 60.51 \\
\hline 26.00 & 61.89 & 80.90 & 83.44 & 83.58 & 83.62 & 83.62 & 83.53 & 83.22 & 72.57 & 60.74 \\
\hline 27.00 & 62.03 & 81.04 & 83.58 & 83.72 & 83.77 & 83.76 & 83.67 & 83.36 & 72.73 & 60.91 \\
\hline 28.00 & 62.18 & 81.36 & 83.91 & 84.05 & 84.10 & 84.10 & 84.01 & 83.69 & 72.98 & 61.02 \\
\hline 29.00 & 62.32 & 81.51 & 84.06 & 84.21 & 84.25 & 84.25 & 84.16 & 83.84 & 73.13 & 61.11 \\
\hline 30.00 & 62.42 & 81.62 & 84.17 & 84.32 & 84.37 & 84.37 & 84.27 & 83.95 & 73.25 & 61.20 \\
\hline 31.00 & 62.53 & 81.77 & 84.32 & 84.47 & 84.52 & 84.51 & 84.42 & 84.10 & 73.41 & 61.35 \\
\hline 32.00 & 62.57 & 81.58 & 84.11 & 84.26 & 84.31 & 84.30 & 84.21 & 83.88 & 73.33 & 61.40 \\
\hline 33.00 & 62.67 & 81.72 & 84.25 & 84.40 & 84.45 & 84.45 & 84.35 & 84.03 & 73.47 & 61.47 \\
\hline 34.00 & 62.75 & 81.95 & 84.49 & 84.65 & 84.70 & 84.69 & 84.59 & 84.27 & 73.67 & 61.54 \\
\hline 35.00 & 62.83 & 82.08 & 84.61 & 84.77 & 84.82 & 84.82 & 84.72 & 84.39 & 73.81 & 61.66 \\
\hline 36.00 & 62.86 & 82.10 & 84.63 & 84.79 & 84.84 & 84.84 & 84.74 & 84.41 & 73.86 & 61.73 \\
\hline 37.00 & 62.92 & 82.23 & 84.77 & 84.93 & 84.98 & 84.98 & 84.87 & 84.54 & 73.99 & 61.76 \\
\hline 38.00 & 62.89 & 81.95 & 84.45 & 84.61 & 84.67 & 84.66 & 84.56 & 84.23 & 73.82 & 61.69 \\
\hline 39.00 & 62.92 & 82.06 & 84.56 & 84.73 & 84.78 & 84.78 & 84.67 & 84.34 & 73.93 & 61.72 \\
\hline 40.00 & 62.78 & 81.16 & 83.59 & 83.75 & 83.81 & 83.80 & 83.69 & 83.37 & 73.37 & 61.64 \\
\hline 41.00 & 62.79 & 81.26 & 83.69 & 83.85 & 83.91 & 83.90 & 83.80 & 83.47 & 73.46 & 61.65 \\
\hline 42.00 & 62.53 & 79.78 & 82.07 & 82.24 & 82.29 & 82.29 & 82.18 & 81.86 & 72.50 & 61.43 \\
\hline 43.00 & 62.50 & 79.86 & 82.15 & 82.32 & 82.38 & 82.37 & 82.26 & 81.94 & 72.57 & 61.40 \\
\hline 44.00 & 59.75 & 60.35 & 60.71 & 60.87 & 60.94 & 60.94 & 60.80 & 60.57 & 60.06 & 59.54 \\
\hline 45.00 & 59.21 & 59.93 & 60.16 & 60.31 & 60.38 & 60.37 & 60.24 & 60.06 & 59.71 & 59.10 \\
\hline
\end{tabular}


Table 37. Temperature $\left({ }^{\circ} \mathrm{C}\right)$ map of the west side of plate 2BZ at EOC 142B (132.4 EFPD).

\begin{tabular}{|c||c|c|c|c|c|c|c|c|c|c|}
\hline \multirow{2}{*}{$\begin{array}{c}\text { Length of } \\
\text { Plate (in) }\end{array}$} & \multicolumn{10}{|c|}{ Width of Plate (inches) } \\
\hline 22.625 & 58.57 & 57.75 & 57.89 & 58.01 & 58.06 & 58.06 & 57.94 & 57.79 & 57.74 & 57.99 \\
\hline 23.00 & 59.81 & 60.49 & 61.18 & 61.32 & 61.38 & 61.37 & 61.26 & 60.99 & 59.58 & 58.84 \\
\hline 24.00 & 63.67 & 81.45 & 83.95 & 84.10 & 84.15 & 84.15 & 84.05 & 83.72 & 73.07 & 61.42 \\
\hline 25.00 & 63.90 & 81.75 & 84.26 & 84.42 & 84.47 & 84.47 & 84.36 & 84.03 & 73.36 & 61.63 \\
\hline 26.00 & 64.13 & 82.48 & 85.04 & 85.20 & 85.25 & 85.25 & 85.14 & 84.80 & 73.93 & 61.93 \\
\hline 27.00 & 64.30 & 82.70 & 85.25 & 85.42 & 85.47 & 85.47 & 85.36 & 85.02 & 74.16 & 62.15 \\
\hline 28.00 & 64.48 & 83.09 & 85.66 & 85.83 & 85.89 & 85.88 & 85.77 & 85.42 & 74.48 & 62.24 \\
\hline 29.00 & 64.65 & 83.33 & 85.90 & 86.07 & 86.13 & 86.13 & 86.01 & 85.66 & 74.71 & 62.29 \\
\hline 30.00 & 64.77 & 83.54 & 86.11 & 86.29 & 86.35 & 86.34 & 86.22 & 85.87 & 74.92 & 62.40 \\
\hline 31.00 & 64.90 & 83.75 & 86.32 & 86.50 & 86.56 & 86.55 & 86.43 & 86.08 & 75.13 & 62.56 \\
\hline 32.00 & 64.93 & 83.64 & 86.19 & 86.37 & 86.43 & 86.43 & 86.30 & 85.95 & 75.12 & 62.59 \\
\hline 33.00 & 65.05 & 83.87 & 86.43 & 86.61 & 86.67 & 86.66 & 86.53 & 86.18 & 75.34 & 62.65 \\
\hline 34.00 & 65.12 & 84.18 & 86.74 & 86.93 & 86.99 & 86.98 & 86.85 & 86.49 & 75.60 & 62.73 \\
\hline 35.00 & 65.22 & 84.37 & 86.94 & 87.13 & 87.19 & 87.19 & 87.06 & 86.69 & 75.80 & 62.90 \\
\hline 36.00 & 65.22 & 84.47 & 87.04 & 87.22 & 87.29 & 87.28 & 87.15 & 86.78 & 75.92 & 62.98 \\
\hline 37.00 & 65.30 & 84.68 & 87.25 & 87.44 & 87.51 & 87.50 & 87.37 & 87.00 & 76.12 & 62.98 \\
\hline 38.00 & 65.22 & 84.47 & 87.01 & 87.20 & 87.27 & 87.26 & 87.12 & 86.76 & 76.01 & 62.84 \\
\hline 39.00 & 65.26 & 84.65 & 87.19 & 87.38 & 87.45 & 87.45 & 87.31 & 86.94 & 76.18 & 62.86 \\
\hline 40.00 & 65.04 & 83.83 & 86.30 & 86.49 & 86.56 & 86.55 & 86.41 & 86.04 & 75.68 & 62.75 \\
\hline 41.00 & 65.05 & 83.99 & 86.46 & 86.65 & 86.72 & 86.72 & 86.57 & 86.20 & 75.83 & 62.77 \\
\hline 42.00 & 64.70 & 82.60 & 84.93 & 85.13 & 85.20 & 85.19 & 85.05 & 84.68 & 74.94 & 62.47 \\
\hline 43.00 & 64.66 & 82.73 & 85.06 & 85.26 & 85.33 & 85.33 & 85.18 & 84.81 & 75.05 & 62.45 \\
\hline 44.00 & 61.30 & 62.73 & 63.14 & 63.32 & 63.41 & 63.40 & 63.23 & 62.96 & 62.37 & 60.30 \\
\hline 45.00 & 60.65 & 62.32 & 62.60 & 62.78 & 62.86 & 62.85 & 62.68 & 62.47 & 62.03 & 59.83 \\
\hline
\end{tabular}




\section{REFERENCES}

1. D.M. Wachs, "RERTR Fuel Development and Qualification Plan," INL/EXT-05-01017, Revision 4, August 2009.

2. "Experiment Control Plan for the AFIP-2 Fuel Irradiation in the ATR," PLN-2689, January 2008.

3. M. A. Lillo, G. S. Chang. "As-Run Neutronic Analys is for the AFIP-2 Experiment Irradiated in the Center Flux Trap of the ATR, Cycles 141A, 142A, and 142B," ECAR-1434. March 2011.

4. RERTR Project Personnel, "RERTR AFIP-2 Irradiation Experiment in the Advanced Test Reactor: As-Bu ilt Data Package," 2600687, March 2008.

5. N.E. Woolstenhulme email to D.M. Perez "AFIP dwgs," April 2010.

6. G. A. Moore, "Fuel Loading Calculations for AFIP-2 Irradiation Experiment," ECA R-132, January 2008.

7. D.M. Wachs, "Hydraulic Testing of the AFIP Irradiation Vehicle for the ATR Center Flux Trap Position," ECAR-99. October 2007.

8. R. G. A mbrosek, "RELAP Analys is for AFIP-2 Flow Resistor," ECA R-126, January 2008.

9. P. E. Murray, "Va lidation of ABAQUS Standard 6.7-3 Heat Transfer", ECA R-131, January 2008.

10. G. A. Roth, "AFIP-2 Thermal As-Run Analysis Results," GA R-01-11, April 2011. 


\section{Appendix A}

\section{Individual Plate Power and Fission Density Plots}




\section{Appendix A \\ Power and Fission Density Plots}

A-1. Plate 2TT

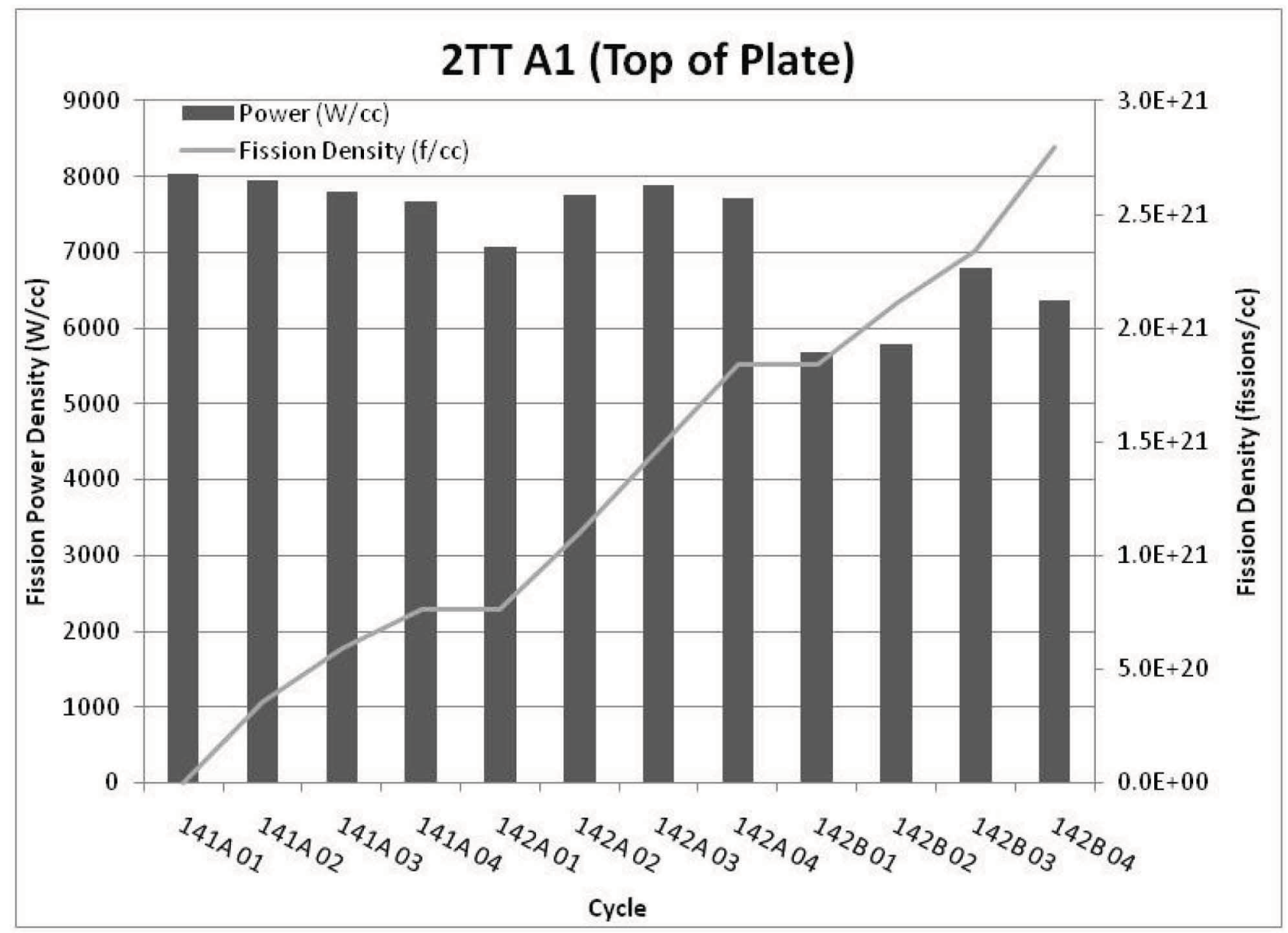



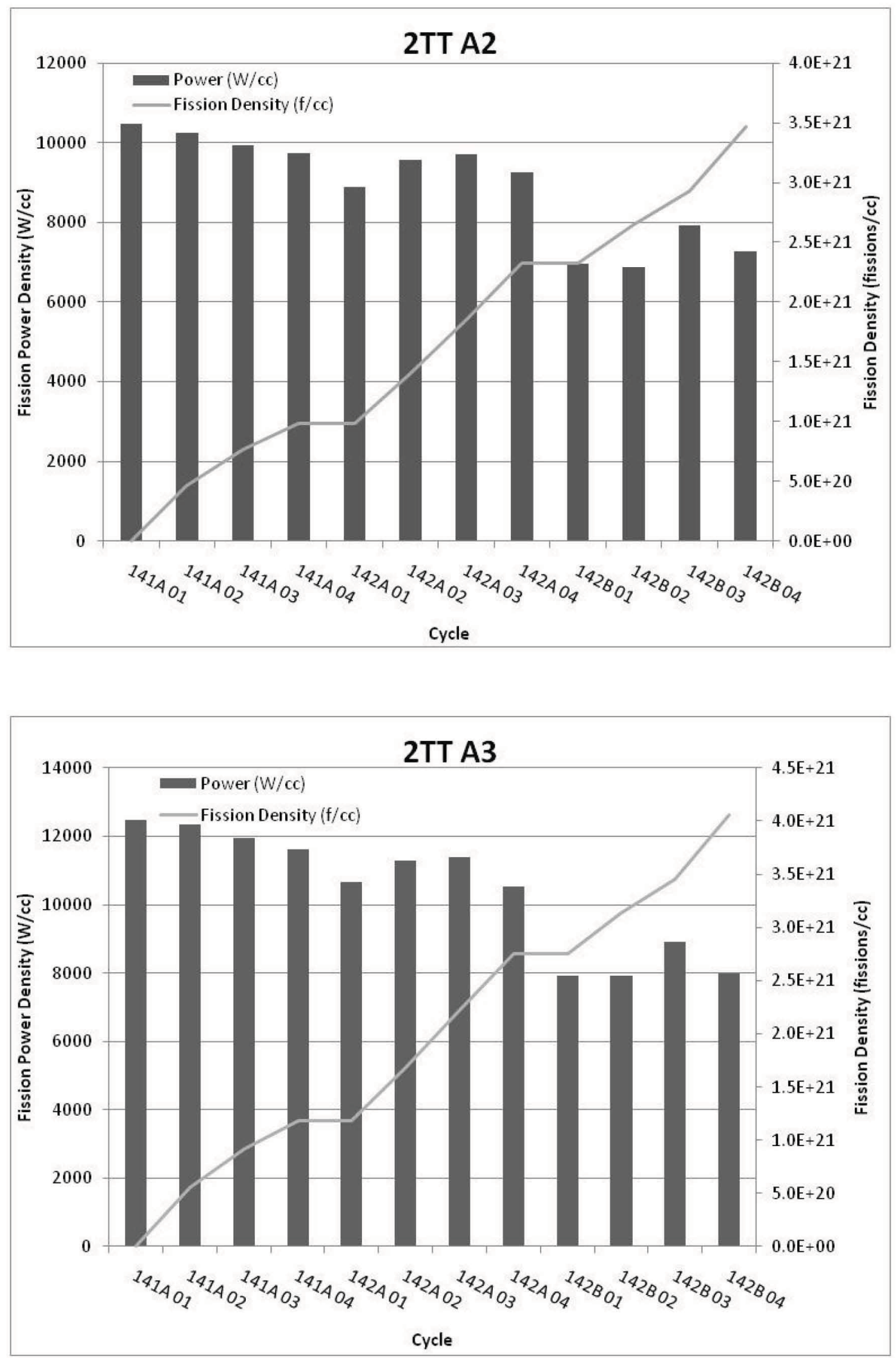

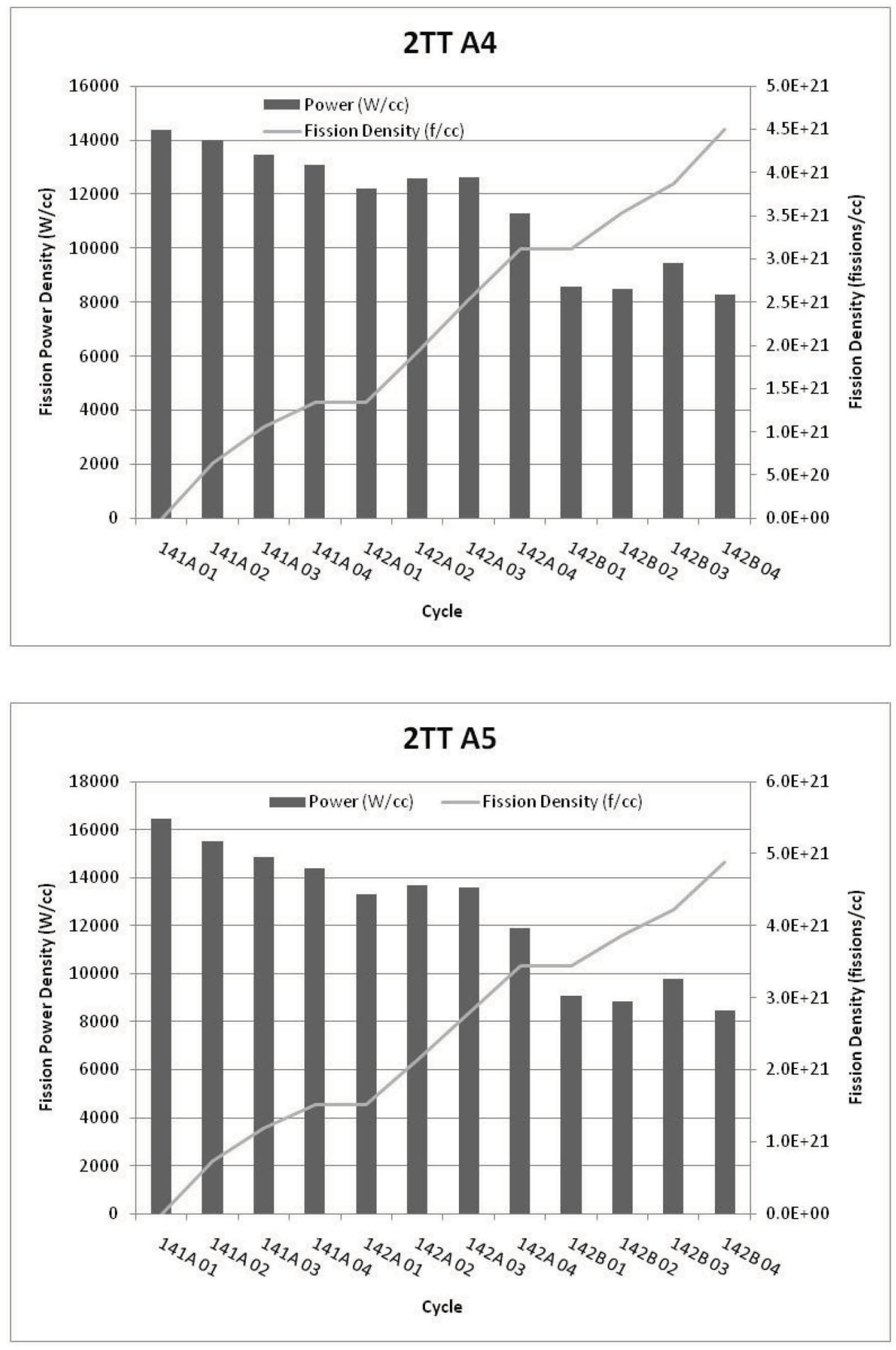

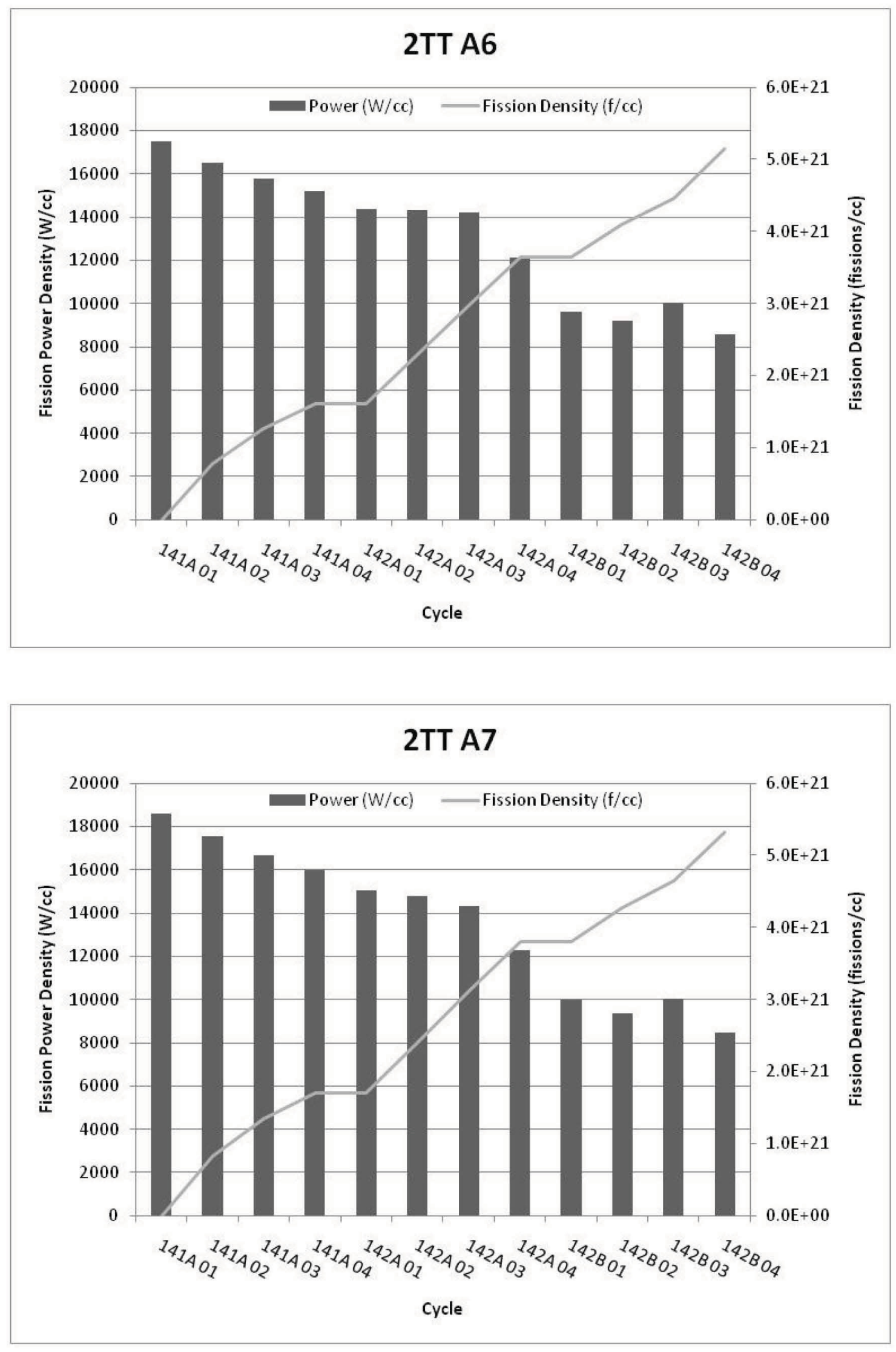

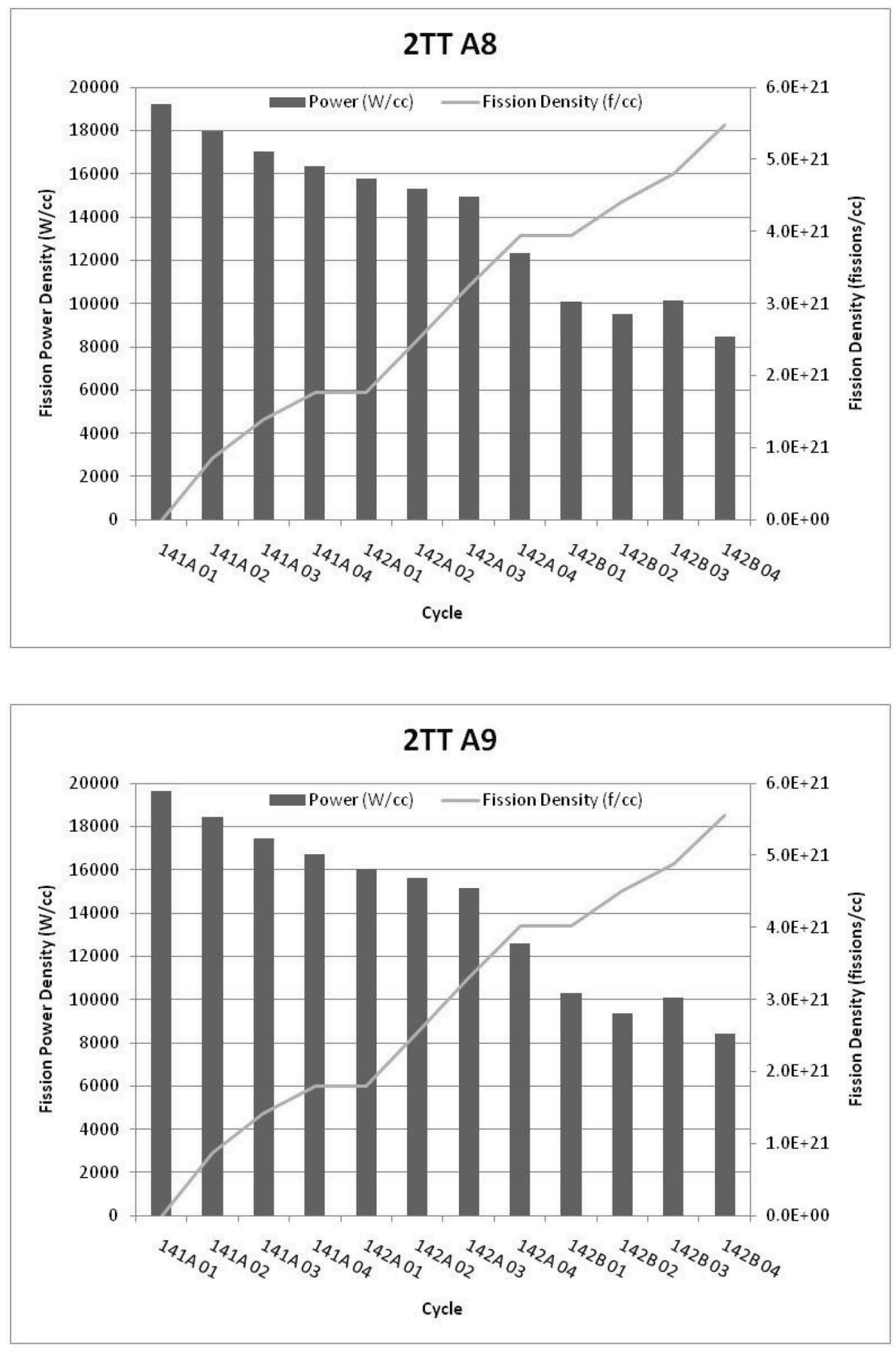


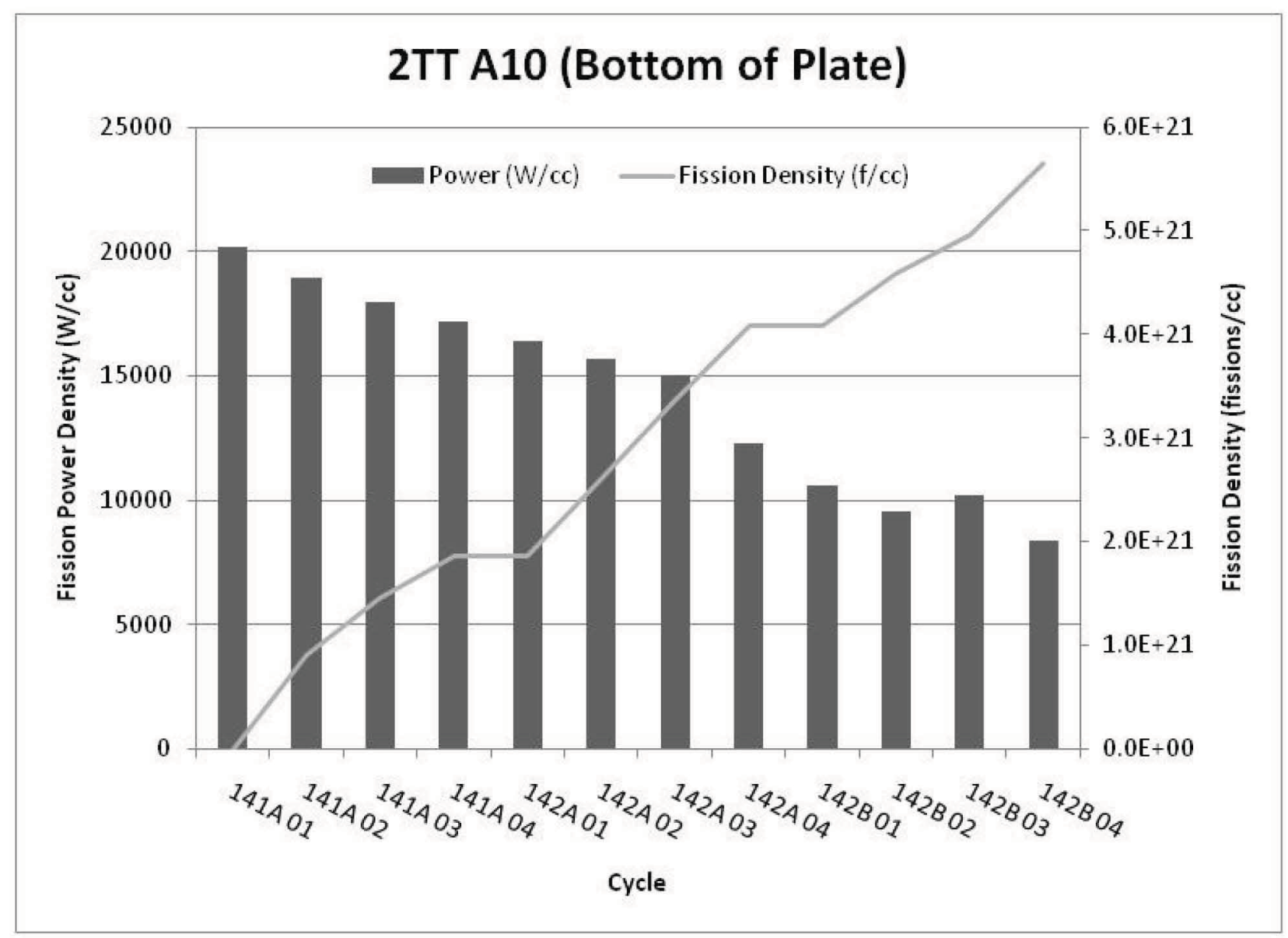




\section{A-2. Plate 2BZ}
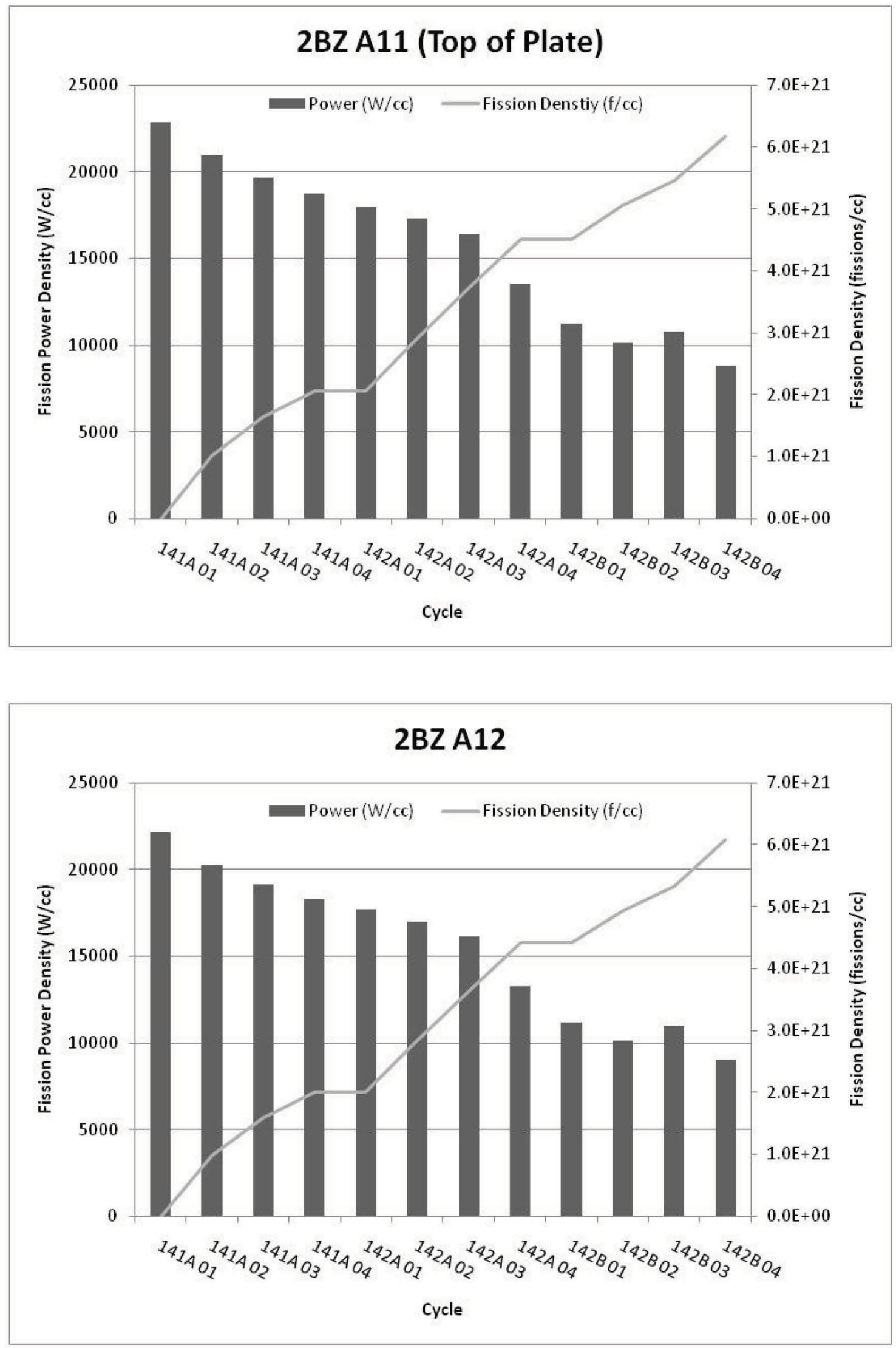

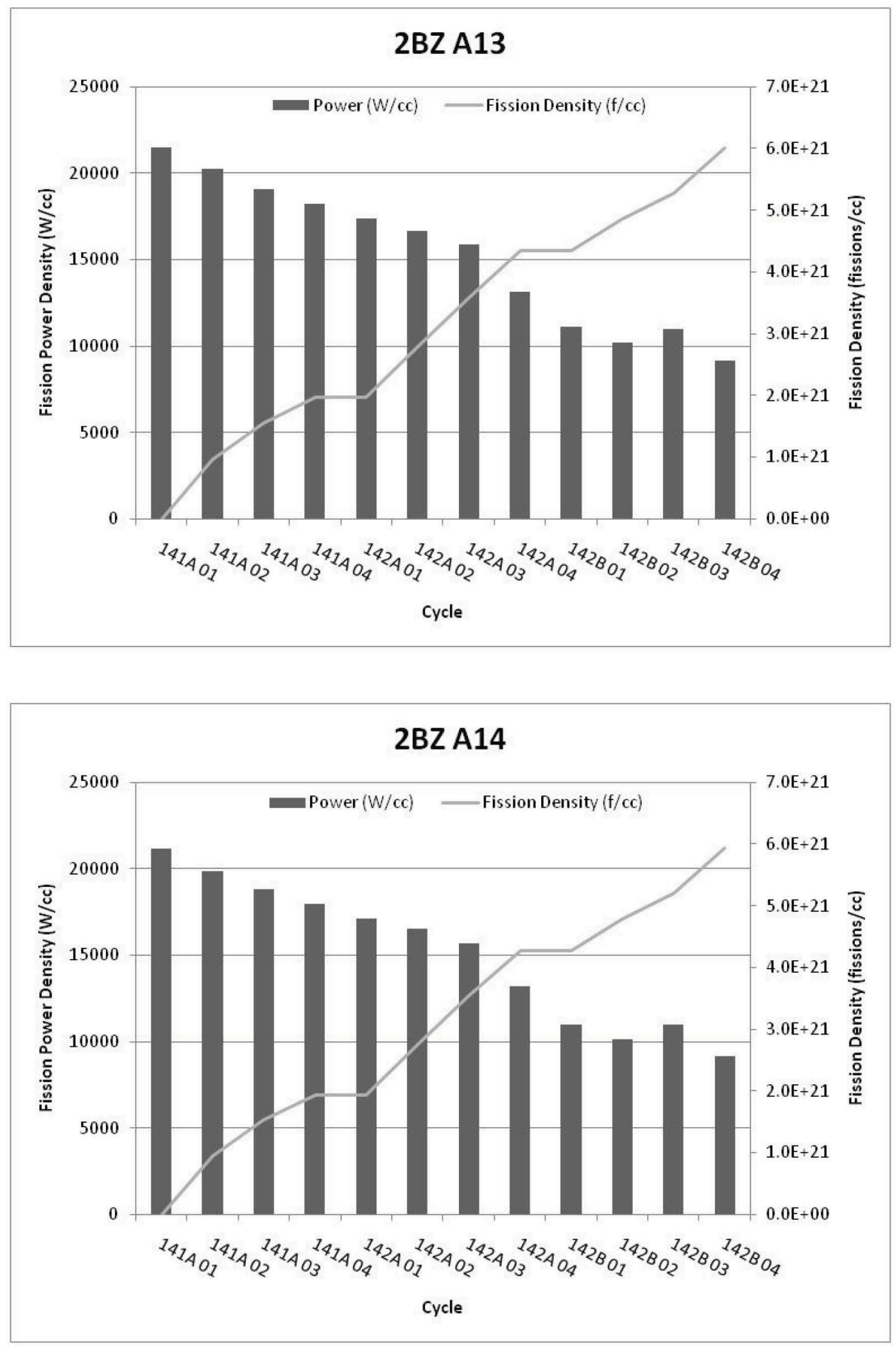

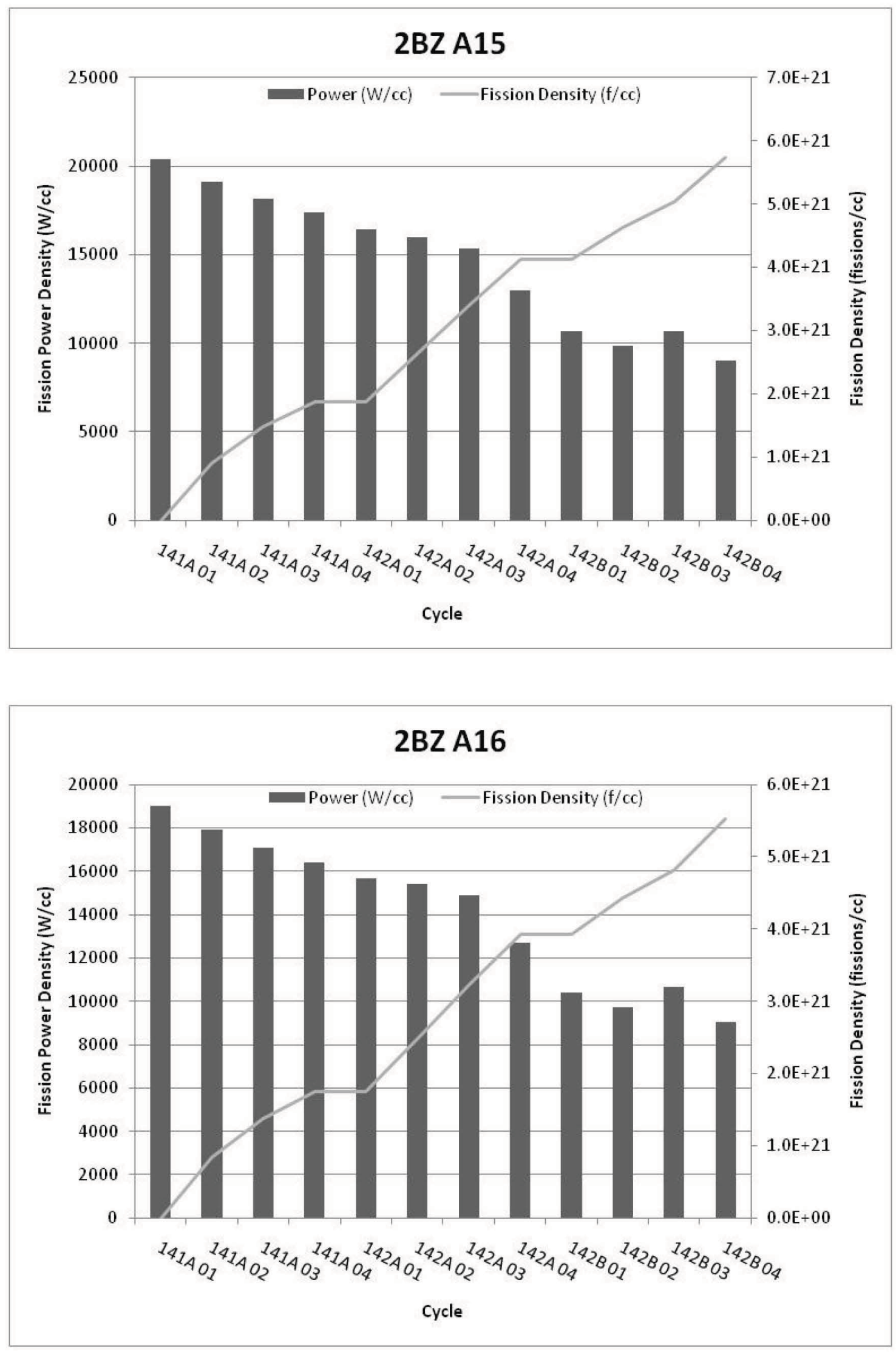

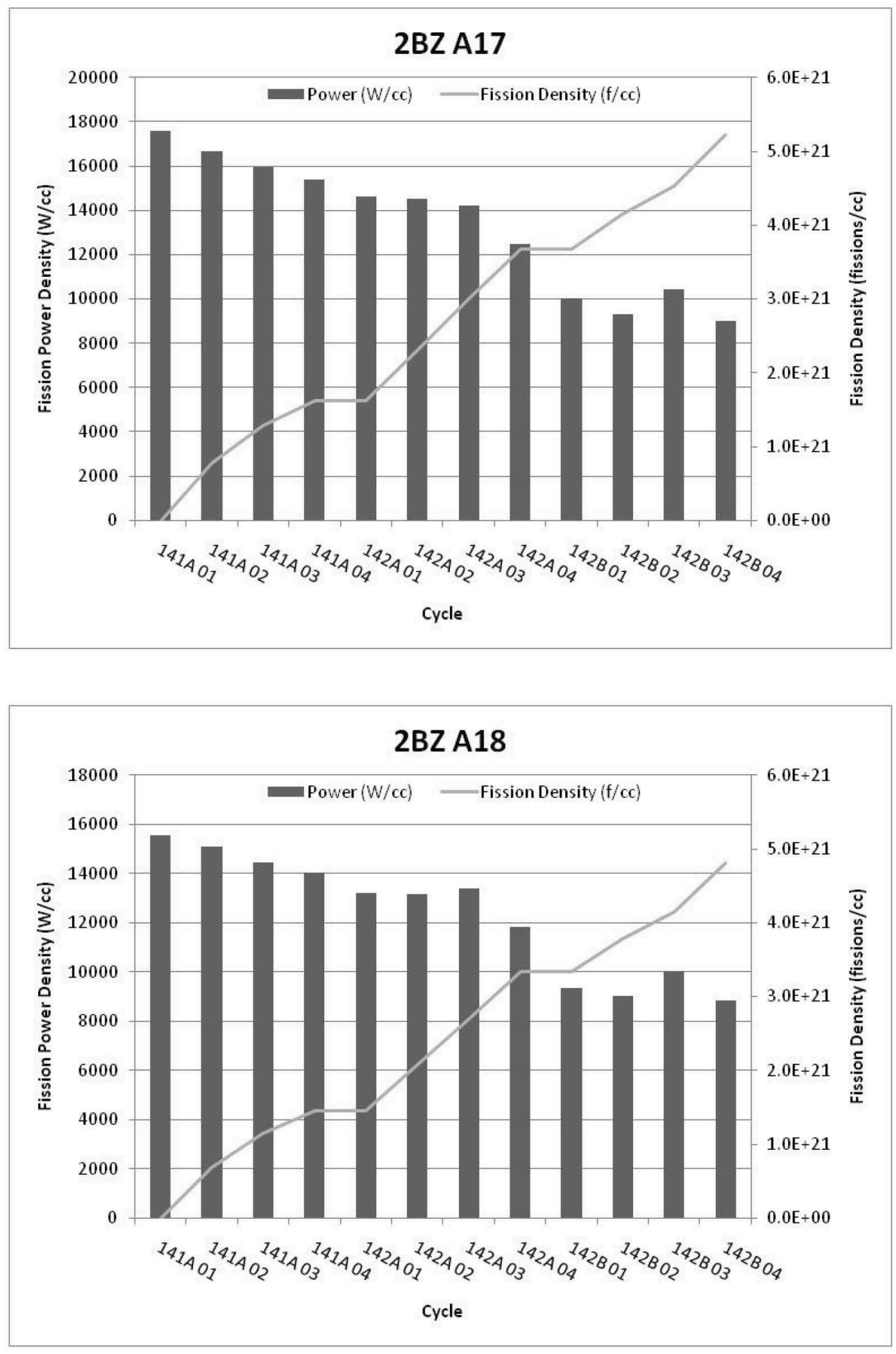

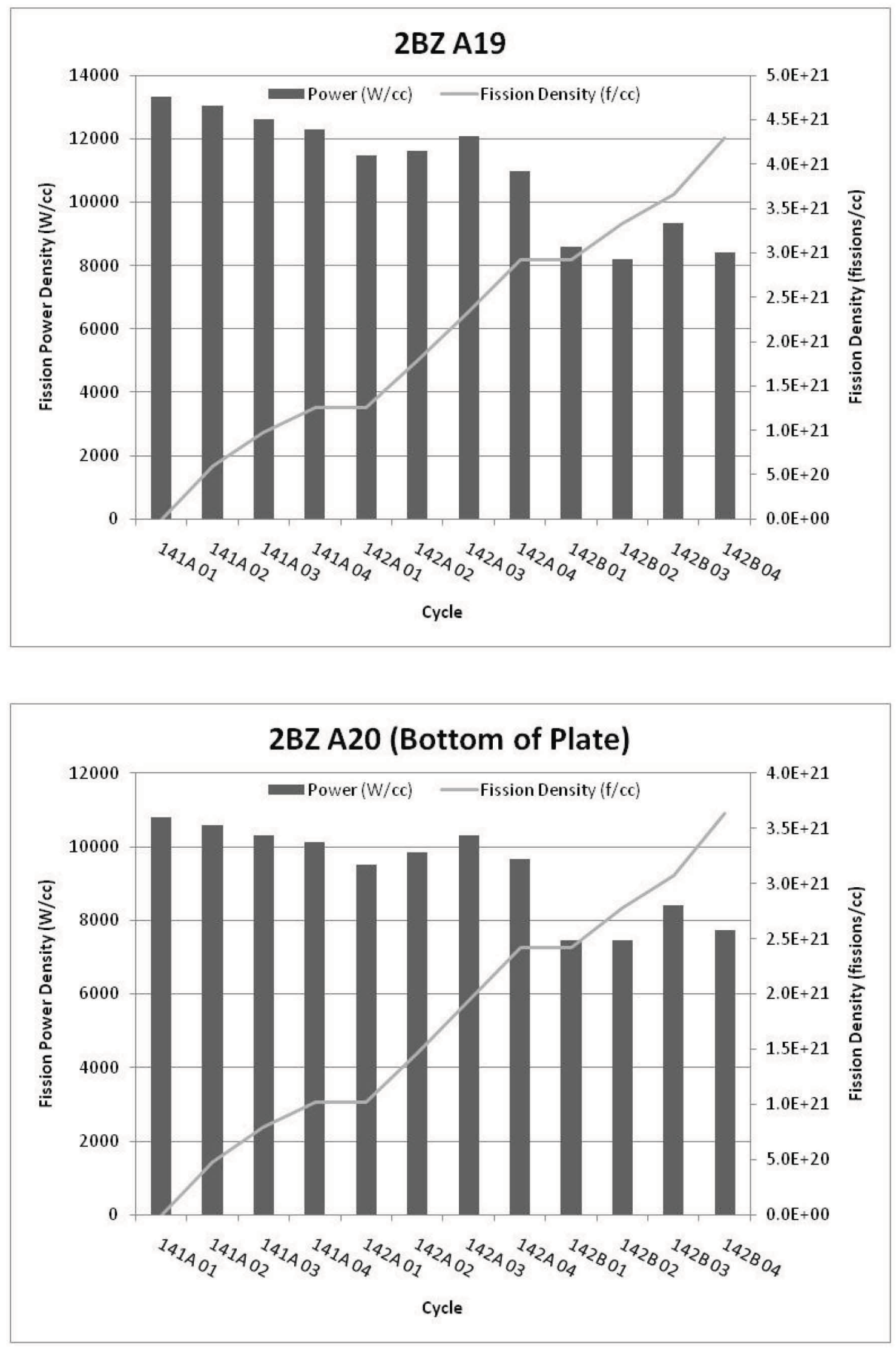


\section{Appendix B}

\section{Plate Surface Temperatures}




\section{Appendix B}

Table B - 1. Temperature $\left({ }^{\circ} \mathrm{C}\right)$ map of the east side of plate 2 TT at BOC 141A (0 EFPD).

\begin{tabular}{|c||c|c|c|c|c|c|c|c|c|c|}
\hline \multirow{2}{*}{$\begin{array}{c}\text { Length of } \\
\text { Plate (in) }\end{array}$} & \multicolumn{10}{|c|}{ Width of Plate (inches) } \\
\hline 0.125 & 51.92 & 51.77 & 51.77 & 51.76 & 51.76 & 51.76 & 51.76 & 51.77 & 51.80 & 51.93 \\
\hline 1.00 & 52.93 & 52.50 & 52.69 & 52.71 & 52.72 & 52.72 & 52.71 & 52.63 & 52.42 & 52.82 \\
\hline 2.00 & 56.89 & 82.62 & 85.72 & 85.80 & 85.80 & 85.80 & 85.79 & 85.52 & 71.82 & 55.39 \\
\hline 3.00 & 57.24 & 82.89 & 86.03 & 86.11 & 86.11 & 86.11 & 86.10 & 85.81 & 72.11 & 55.71 \\
\hline 4.00 & 58.55 & 90.83 & 94.59 & 94.68 & 94.69 & 94.69 & 94.67 & 94.35 & 77.57 & 56.62 \\
\hline 5.00 & 58.84 & 91.03 & 94.80 & 94.90 & 94.90 & 94.90 & 94.88 & 94.56 & 77.79 & 56.89 \\
\hline 6.00 & 60.00 & 97.27 & 101.61 & 101.72 & 101.73 & 101.73 & 101.70 & 101.32 & 82.17 & 57.69 \\
\hline 7.00 & 60.27 & 97.47 & 101.85 & 101.97 & 101.98 & 101.98 & 101.95 & 101.55 & 82.38 & 57.94 \\
\hline 8.00 & 61.46 & 103.56 & 108.63 & 108.76 & 108.78 & 108.78 & 108.74 & 108.29 & 86.56 & 58.79 \\
\hline 9.00 & 61.73 & 103.81 & 108.88 & 109.02 & 109.04 & 109.04 & 109.00 & 108.54 & 86.81 & 59.11 \\
\hline 10.00 & 62.95 & 110.29 & 115.90 & 116.06 & 116.08 & 116.08 & 116.03 & 115.53 & 91.13 & 59.96 \\
\hline 11.00 & 63.24 & 110.56 & 116.18 & 116.34 & 116.36 & 116.36 & 116.31 & 115.80 & 91.38 & 60.21 \\
\hline 12.00 & 64.05 & 114.02 & 119.91 & 120.08 & 120.11 & 120.11 & 120.05 & 119.52 & 93.73 & 60.83 \\
\hline 13.00 & 64.33 & 114.29 & 120.19 & 120.36 & 120.40 & 120.40 & 120.33 & 119.78 & 93.99 & 61.11 \\
\hline 14.00 & 65.07 & 117.65 & 123.81 & 123.99 & 124.03 & 124.03 & 123.95 & 123.39 & 96.26 & 61.66 \\
\hline 15.00 & 65.37 & 117.93 & 124.09 & 124.29 & 124.32 & 124.32 & 124.24 & 123.67 & 96.52 & 61.93 \\
\hline 16.00 & 65.98 & 120.16 & 126.48 & 126.68 & 126.72 & 126.72 & 126.64 & 126.04 & 98.12 & 62.38 \\
\hline 17.00 & 66.27 & 120.43 & 126.76 & 126.97 & 127.01 & 127.01 & 126.92 & 126.32 & 98.42 & 62.71 \\
\hline 18.00 & 66.71 & 121.87 & 128.30 & 128.51 & 128.56 & 128.56 & 128.46 & 127.85 & 99.52 & 63.09 \\
\hline 19.00 & 67.00 & 122.15 & 128.59 & 128.81 & 128.86 & 128.86 & 128.75 & 128.13 & 99.81 & 63.34 \\
\hline 20.00 & 67.52 & 123.98 & 130.54 & 130.77 & 130.82 & 130.82 & 130.71 & 130.08 & 101.18 & 63.73 \\
\hline 21.00 & 67.78 & 124.24 & 130.79 & 131.03 & 131.09 & 131.09 & 130.97 & 130.33 & 101.45 & 63.96 \\
\hline 22.00 & 60.55 & 66.78 & 68.79 & 69.00 & 69.08 & 69.07 & 68.92 & 68.34 & 63.77 & 59.52 \\
\hline 22.50 & 58.23 & 58.64 & 59.02 & 59.19 & 59.27 & 59.26 & 59.11 & 58.83 & 58.44 & 57.86 \\
\hline
\end{tabular}


Table B - 2.Temperature $\left({ }^{\circ} \mathrm{C}\right)$ map of the west side of plate 2 TT at BOC $141 \mathrm{~A}(0$ EFPD).

\begin{tabular}{|c||c|c|c|c|c|c|c|c|c|c|}
\hline \multirow{2}{*}{$\begin{array}{c}\text { Length of } \\
\text { Plate (in) }\end{array}$} & \multicolumn{10}{|c|}{ Width of Plate (inches) } \\
\hline 0.125 & 51.95 & 51.96 & 51.77 & 51.77 & 51.76 & 51.76 & 51.76 & 51.76 & 51.77 & 51.80 \\
\hline 1.00 & 52.92 & 53.06 & 52.50 & 52.69 & 52.71 & 52.72 & 52.71 & 52.71 & 52.63 & 52.42 \\
\hline 2.00 & 55.62 & 57.22 & 82.61 & 85.72 & 85.79 & 85.80 & 85.80 & 85.79 & 85.52 & 71.81 \\
\hline 3.00 & 55.97 & 57.59 & 82.89 & 86.03 & 86.10 & 86.11 & 86.11 & 86.10 & 85.81 & 72.10 \\
\hline 4.00 & 56.91 & 58.97 & 90.83 & 94.59 & 94.68 & 94.68 & 94.68 & 94.67 & 94.35 & 77.55 \\
\hline 5.00 & 57.19 & 59.27 & 91.03 & 94.80 & 94.89 & 94.90 & 94.90 & 94.88 & 94.55 & 77.78 \\
\hline 6.00 & 58.05 & 60.48 & 97.27 & 101.60 & 101.71 & 101.72 & 101.72 & 101.70 & 101.31 & 82.16 \\
\hline 7.00 & 58.37 & 60.81 & 97.51 & 101.88 & 102.00 & 102.01 & 102.01 & 101.98 & 101.59 & 82.41 \\
\hline 8.00 & 59.21 & 62.01 & 103.57 & 108.62 & 108.75 & 108.77 & 108.76 & 108.73 & 108.29 & 86.55 \\
\hline 9.00 & 59.49 & 62.30 & 103.82 & 108.87 & 109.01 & 109.03 & 109.02 & 108.99 & 108.54 & 86.80 \\
\hline 10.00 & 60.39 & 63.58 & 110.31 & 115.89 & 116.04 & 116.06 & 116.06 & 116.02 & 115.53 & 91.13 \\
\hline 11.00 & 60.72 & 63.92 & 110.61 & 116.20 & 116.36 & 116.38 & 116.38 & 116.33 & 115.83 & 91.41 \\
\hline 12.00 & 61.31 & 64.71 & 114.04 & 119.90 & 120.06 & 120.09 & 120.08 & 120.04 & 119.52 & 93.73 \\
\hline 13.00 & 61.58 & 65.00 & 114.31 & 120.18 & 120.34 & 120.37 & 120.37 & 120.31 & 119.79 & 93.99 \\
\hline 14.00 & 62.16 & 65.77 & 117.67 & 123.80 & 123.97 & 124.00 & 124.00 & 123.94 & 123.39 & 96.27 \\
\hline 15.00 & 62.45 & 66.08 & 117.95 & 124.08 & 124.26 & 124.29 & 124.29 & 124.23 & 123.67 & 96.53 \\
\hline 16.00 & 62.94 & 66.70 & 120.18 & 126.47 & 126.65 & 126.69 & 126.68 & 126.62 & 126.05 & 98.13 \\
\hline 17.00 & 63.22 & 66.99 & 120.46 & 126.75 & 126.94 & 126.98 & 126.97 & 126.90 & 126.32 & 98.44 \\
\hline 18.00 & 63.58 & 67.44 & 121.90 & 128.28 & 128.48 & 128.52 & 128.52 & 128.44 & 127.85 & 99.54 \\
\hline 19.00 & 63.86 & 67.74 & 122.19 & 128.57 & 128.78 & 128.82 & 128.81 & 128.73 & 128.14 & 99.84 \\
\hline 20.00 & 64.28 & 68.27 & 124.02 & 130.52 & 130.73 & 130.78 & 130.77 & 130.69 & 130.08 & 101.21 \\
\hline 21.00 & 64.52 & 68.53 & 124.27 & 130.78 & 130.99 & 131.04 & 131.04 & 130.94 & 130.34 & 101.48 \\
\hline 22.00 & 59.87 & 60.93 & 66.81 & 68.78 & 68.97 & 69.03 & 69.03 & 68.90 & 68.34 & 63.82 \\
\hline 22.50 & 58.15 & 58.57 & 58.68 & 59.01 & 59.16 & 59.22 & 59.21 & 59.09 & 58.84 & 58.49 \\
\hline
\end{tabular}


Table B - 3.Temperature $\left({ }^{\circ} \mathrm{C}\right)$ map of the east side of plate $2 \mathrm{BZ}$ at BOC $141 \mathrm{~A}(0 \mathrm{EFPD})$.

\begin{tabular}{|c||c|c|c|c|c|c|c|c|c|c|}
\hline \multirow{2}{*}{$\begin{array}{c}\text { Length of } \\
\text { Plate (in) }\end{array}$} & \multicolumn{10}{|c|}{ Width of Plate (inches) } \\
\hline 22.625 & 58.40 & 58.70 & 59.08 & 59.26 & 59.33 & 59.33 & 59.18 & 58.90 & 58.49 & 58.01 \\
\hline 23.00 & 60.22 & 65.27 & 66.97 & 67.18 & 67.25 & 67.25 & 67.09 & 66.57 & 62.80 & 59.30 \\
\hline 24.00 & 66.40 & 113.03 & 118.62 & 118.86 & 118.93 & 118.93 & 118.79 & 118.20 & 93.97 & 63.21 \\
\hline 25.00 & 66.60 & 113.23 & 118.81 & 119.06 & 119.13 & 119.13 & 118.98 & 118.38 & 94.18 & 63.40 \\
\hline 26.00 & 66.61 & 112.12 & 117.61 & 117.86 & 117.94 & 117.93 & 117.78 & 117.18 & 93.55 & 63.50 \\
\hline 27.00 & 66.77 & 112.34 & 117.83 & 118.09 & 118.16 & 118.16 & 118.01 & 117.40 & 93.76 & 63.66 \\
\hline 28.00 & 66.77 & 111.26 & 116.64 & 116.91 & 116.99 & 116.98 & 116.82 & 116.21 & 93.11 & 63.70 \\
\hline 29.00 & 66.94 & 111.48 & 116.86 & 117.13 & 117.21 & 117.20 & 117.04 & 116.43 & 93.32 & 63.82 \\
\hline 30.00 & 67.01 & 110.94 & 116.26 & 116.54 & 116.62 & 116.62 & 116.45 & 115.83 & 93.05 & 63.92 \\
\hline 31.00 & 67.15 & 111.15 & 116.47 & 116.74 & 116.83 & 116.83 & 116.65 & 116.03 & 93.26 & 64.06 \\
\hline 32.00 & 67.07 & 109.79 & 114.98 & 115.25 & 115.34 & 115.34 & 115.16 & 114.54 & 92.41 & 64.05 \\
\hline 33.00 & 67.20 & 109.98 & 115.17 & 115.45 & 115.54 & 115.54 & 115.36 & 114.74 & 92.61 & 64.15 \\
\hline 34.00 & 66.88 & 107.18 & 112.11 & 112.40 & 112.49 & 112.48 & 112.30 & 111.68 & 90.83 & 63.98 \\
\hline 35.00 & 66.98 & 107.35 & 112.29 & 112.58 & 112.67 & 112.67 & 112.48 & 111.86 & 91.00 & 64.09 \\
\hline 36.00 & 66.63 & 104.46 & 109.13 & 109.42 & 109.52 & 109.51 & 109.32 & 108.71 & 89.18 & 63.91 \\
\hline 37.00 & 66.71 & 104.63 & 109.29 & 109.58 & 109.68 & 109.68 & 109.48 & 108.88 & 89.33 & 63.97 \\
\hline 38.00 & 66.15 & 100.39 & 104.67 & 104.96 & 105.06 & 105.06 & 104.86 & 104.27 & 86.63 & 63.60 \\
\hline 39.00 & 66.20 & 100.53 & 104.80 & 105.09 & 105.20 & 105.19 & 104.99 & 104.40 & 86.76 & 63.63 \\
\hline 40.00 & 65.54 & 95.64 & 99.46 & 99.75 & 99.86 & 99.85 & 99.64 & 99.08 & 83.64 & 63.23 \\
\hline 41.00 & 65.54 & 95.74 & 99.55 & 99.84 & 99.95 & 99.94 & 99.73 & 99.17 & 83.73 & 63.23 \\
\hline 42.00 & 64.74 & 90.07 & 93.34 & 93.63 & 93.74 & 93.74 & 93.52 & 92.99 & 80.09 & 62.71 \\
\hline 43.00 & 64.70 & 90.14 & 93.40 & 93.69 & 93.80 & 93.79 & 93.58 & 93.05 & 80.15 & 62.67 \\
\hline 44.00 & 61.37 & 63.82 & 64.47 & 64.73 & 64.85 & 64.84 & 64.61 & 64.22 & 63.24 & 60.57 \\
\hline 45.00 & 60.80 & 63.25 & 63.72 & 63.97 & 64.08 & 64.08 & 63.85 & 63.53 & 62.79 & 60.10 \\
\hline
\end{tabular}


Table B - 4. Temperature $\left({ }^{\circ} \mathrm{C}\right)$ map of the west side of plate $2 \mathrm{BZ}$ at BOC $141 \mathrm{~A}(0 \mathrm{EFPD})$.

\begin{tabular}{|c||c|c|c|c|c|c|c|c|c|c|}
\hline \multirow{2}{*}{$\begin{array}{c}\text { Length of } \\
\text { Plate (in) }\end{array}$} & \multicolumn{10}{|c|}{ Width of Plate (inches) } \\
\hline 22.625 & 58.27 & 58.67 & 58.73 & 59.07 & 59.22 & 59.29 & 59.28 & 59.15 & 58.90 & 58.54 \\
\hline 23.00 & 59.64 & 60.57 & 65.31 & 66.96 & 67.14 & 67.21 & 67.20 & 67.07 & 66.58 & 62.85 \\
\hline 24.00 & 63.75 & 67.09 & 113.12 & 118.65 & 118.87 & 118.92 & 118.91 & 118.81 & 118.25 & 94.04 \\
\hline 25.00 & 63.90 & 67.24 & 113.27 & 118.79 & 119.01 & 119.07 & 119.06 & 118.95 & 118.39 & 94.22 \\
\hline 26.00 & 63.96 & 67.25 & 112.17 & 117.59 & 117.81 & 117.87 & 117.87 & 117.75 & 117.18 & 93.57 \\
\hline 27.00 & 64.11 & 67.40 & 112.39 & 117.81 & 118.04 & 118.10 & 118.09 & 117.97 & 117.40 & 93.80 \\
\hline 28.00 & 64.30 & 67.54 & 111.52 & 116.84 & 117.07 & 117.13 & 117.13 & 117.00 & 116.43 & 93.36 \\
\hline 29.00 & 64.38 & 67.60 & 110.99 & 116.25 & 116.48 & 116.54 & 116.54 & 116.41 & 115.84 & 93.09 \\
\hline 30.00 & 64.50 & 67.73 & 111.20 & 116.45 & 116.69 & 116.75 & 116.75 & 116.61 & 116.04 & 93.30 \\
\hline 31.00 & 64.47 & 67.62 & 109.83 & 114.96 & 115.19 & 115.26 & 115.25 & 115.12 & 114.55 & 92.46 \\
\hline 32.00 & 64.58 & 67.73 & 110.05 & 115.17 & 115.41 & 115.48 & 115.47 & 115.33 & 114.76 & 92.67 \\
\hline 33.00 & 64.39 & 67.38 & 107.23 & 112.09 & 112.33 & 112.40 & 112.40 & 112.25 & 111.69 & 90.87 \\
\hline 34.00 & 64.46 & 67.47 & 107.41 & 112.27 & 112.51 & 112.58 & 112.58 & 112.43 & 111.87 & 91.04 \\
\hline 35.00 & 64.24 & 67.08 & 104.51 & 109.11 & 109.36 & 109.43 & 109.42 & 109.27 & 108.72 & 89.23 \\
\hline 36.00 & 64.30 & 67.15 & 104.68 & 109.28 & 109.52 & 109.59 & 109.59 & 109.44 & 108.88 & 89.37 \\
\hline 37.00 & 63.92 & 66.54 & 100.45 & 104.65 & 104.89 & 104.97 & 104.96 & 104.81 & 104.28 & 86.68 \\
\hline 38.00 & 63.94 & 66.57 & 100.58 & 104.78 & 105.03 & 105.10 & 105.09 & 104.94 & 104.41 & 86.80 \\
\hline 39.00 & 63.50 & 65.86 & 95.69 & 99.44 & 99.68 & 99.76 & 99.75 & 99.59 & 99.09 & 83.69 \\
\hline 40.00 & 63.47 & 65.84 & 95.79 & 99.53 & 99.77 & 99.85 & 99.85 & 99.69 & 99.18 & 83.77 \\
\hline 41.00 & 62.93 & 64.97 & 90.13 & 93.33 & 93.56 & 93.64 & 93.63 & 93.47 & 93.00 & 80.14 \\
\hline 42.00 & 62.87 & 64.92 & 90.19 & 93.38 & 93.62 & 93.70 & 93.69 & 93.53 & 93.06 & 80.20 \\
\hline 43.00 & 60.64 & 61.40 & 63.86 & 64.45 & 64.68 & 64.77 & 64.76 & 64.57 & 64.23 & 63.30 \\
\hline 44.00 & 60.15 & 60.80 & 63.29 & 63.71 & 63.91 & 64.01 & 64.00 & 63.81 & 63.53 & 62.85 \\
\hline 45.00 & 58.27 & 58.67 & 58.73 & 59.07 & 59.22 & 59.29 & 59.28 & 59.15 & 58.90 & 58.54 \\
\hline
\end{tabular}


Table B - 5. Temperature $\left({ }^{\circ} \mathrm{C}\right)$ map of the east side of plate $2 \mathrm{TT}$ at MOC 141A (15 EFPD).

\begin{tabular}{|c||c|c|c|c|c|c|c|c|c|c|}
\hline \multirow{2}{*}{$\begin{array}{c}\text { Length of } \\
\text { Plate (in) }\end{array}$} & 0.05 & 0.25 & 0.50 & 0.75 & 1.00 & 1.25 & 1.50 & 1.75 & 2.00 & 2.24 \\
\hline 0.125 & 51.92 & 51.77 & 51.77 & 51.76 & 51.76 & 51.76 & 51.76 & 51.77 & 51.80 & 51.93 \\
\hline 1.00 & 52.92 & 52.50 & 52.68 & 52.70 & 52.71 & 52.71 & 52.70 & 52.62 & 52.42 & 52.81 \\
\hline 2.00 & 56.84 & 82.31 & 85.39 & 85.47 & 85.47 & 85.47 & 85.46 & 85.19 & 71.62 & 55.36 \\
\hline 3.00 & 57.18 & 82.58 & 85.69 & 85.77 & 85.78 & 85.77 & 85.76 & 85.47 & 71.90 & 55.67 \\
\hline 4.00 & 58.43 & 90.10 & 93.80 & 93.89 & 93.90 & 93.90 & 93.88 & 93.56 & 77.06 & 56.54 \\
\hline 5.00 & 58.72 & 90.30 & 94.01 & 94.10 & 94.11 & 94.11 & 94.09 & 93.77 & 77.28 & 56.81 \\
\hline 6.00 & 59.93 & 96.90 & 101.19 & 101.30 & 101.31 & 101.31 & 101.28 & 100.90 & 81.91 & 57.65 \\
\hline 7.00 & 60.24 & 97.13 & 101.47 & 101.59 & 101.60 & 101.60 & 101.57 & 101.17 & 82.16 & 57.94 \\
\hline 8.00 & 61.26 & 102.38 & 107.35 & 107.48 & 107.50 & 107.50 & 107.46 & 107.02 & 85.78 & 58.66 \\
\hline 9.00 & 61.53 & 102.62 & 107.59 & 107.73 & 107.75 & 107.75 & 107.71 & 107.25 & 86.02 & 58.97 \\
\hline 10.00 & 62.49 & 107.44 & 112.82 & 112.97 & 112.99 & 112.99 & 112.94 & 112.46 & 89.25 & 59.66 \\
\hline 11.00 & 62.81 & 107.72 & 113.11 & 113.26 & 113.29 & 113.29 & 113.23 & 112.74 & 89.52 & 59.93 \\
\hline 12.00 & 63.54 & 111.00 & 116.64 & 116.81 & 116.83 & 116.83 & 116.77 & 116.26 & 91.73 & 60.48 \\
\hline 13.00 & 63.81 & 111.25 & 116.90 & 117.08 & 117.11 & 117.10 & 117.04 & 116.51 & 91.98 & 60.75 \\
\hline 14.00 & 64.52 & 114.49 & 120.39 & 120.57 & 120.60 & 120.60 & 120.53 & 119.98 & 94.17 & 61.29 \\
\hline 15.00 & 64.81 & 114.76 & 120.66 & 120.85 & 120.89 & 120.89 & 120.81 & 120.25 & 94.42 & 61.54 \\
\hline 16.00 & 65.29 & 116.26 & 122.28 & 122.47 & 122.51 & 122.51 & 122.43 & 121.86 & 95.49 & 61.91 \\
\hline 17.00 & 65.56 & 116.52 & 122.54 & 122.74 & 122.79 & 122.79 & 122.70 & 122.12 & 95.75 & 62.23 \\
\hline 18.00 & 66.01 & 118.14 & 124.27 & 124.48 & 124.53 & 124.53 & 124.43 & 123.84 & 96.90 & 62.61 \\
\hline 19.00 & 66.29 & 118.41 & 124.55 & 124.77 & 124.82 & 124.82 & 124.72 & 124.11 & 97.19 & 62.86 \\
\hline 20.00 & 66.78 & 120.12 & 126.37 & 126.60 & 126.65 & 126.65 & 126.54 & 125.93 & 98.46 & 63.22 \\
\hline 21.00 & 67.03 & 120.36 & 126.62 & 126.85 & 126.90 & 126.90 & 126.79 & 126.17 & 98.71 & 63.44 \\
\hline 22.00 & 60.23 & 66.01 & 67.91 & 68.11 & 68.18 & 68.18 & 68.03 & 67.48 & 63.18 & 59.26 \\
\hline 22.50 & 58.05 & 58.33 & 58.69 & 58.85 & 58.92 & 58.91 & 58.77 & 58.51 & 58.16 & 57.70 \\
\hline
\end{tabular}


Table B - 6. Temperature $\left({ }^{\circ} \mathrm{C}\right)$ map of the west side of plate 2 TT at MOC 141A (15 EFPD).

\begin{tabular}{|c||c|c|c|c|c|c|c|c|c|c|}
\hline \multirow{2}{*}{$\begin{array}{c}\text { Length of } \\
\text { Plate (in) }\end{array}$} & \multicolumn{10}{|c|}{ Width of Plate (inches) } \\
\hline 0.125 & 51.96 & 51.77 & 51.77 & 51.76 & 51.76 & 51.76 & 51.76 & 51.77 & 51.80 & 51.95 \\
\hline 1.00 & 53.05 & 52.50 & 52.68 & 52.70 & 52.71 & 52.71 & 52.70 & 52.62 & 52.42 & 52.88 \\
\hline 2.00 & 57.17 & 82.31 & 85.39 & 85.47 & 85.47 & 85.47 & 85.46 & 85.19 & 71.61 & 55.45 \\
\hline 3.00 & 57.53 & 82.58 & 85.69 & 85.77 & 85.77 & 85.77 & 85.76 & 85.47 & 71.89 & 55.77 \\
\hline 4.00 & 58.85 & 90.10 & 93.80 & 93.89 & 93.89 & 93.89 & 93.88 & 93.56 & 77.05 & 56.66 \\
\hline 5.00 & 59.15 & 90.30 & 94.01 & 94.10 & 94.11 & 94.11 & 94.09 & 93.77 & 77.27 & 56.93 \\
\hline 6.00 & 60.42 & 96.91 & 101.18 & 101.29 & 101.30 & 101.30 & 101.28 & 100.90 & 81.90 & 57.78 \\
\hline 7.00 & 60.74 & 97.14 & 101.46 & 101.58 & 101.59 & 101.59 & 101.56 & 101.17 & 82.15 & 58.06 \\
\hline 8.00 & 61.81 & 102.39 & 107.34 & 107.47 & 107.48 & 107.48 & 107.45 & 107.02 & 85.77 & 58.79 \\
\hline 9.00 & 62.09 & 102.63 & 107.58 & 107.72 & 107.73 & 107.73 & 107.70 & 107.25 & 86.01 & 59.16 \\
\hline 10.00 & 63.09 & 107.46 & 112.81 & 112.95 & 112.97 & 112.97 & 112.93 & 112.46 & 89.25 & 59.84 \\
\hline 11.00 & 63.42 & 107.74 & 113.10 & 113.25 & 113.27 & 113.27 & 113.22 & 112.74 & 89.51 & 60.10 \\
\hline 12.00 & 64.18 & 111.02 & 116.63 & 116.79 & 116.81 & 116.81 & 116.76 & 116.26 & 91.73 & 60.64 \\
\hline 13.00 & 64.46 & 111.27 & 116.89 & 117.05 & 117.08 & 117.08 & 117.03 & 116.51 & 91.98 & 60.93 \\
\hline 14.00 & 65.20 & 114.51 & 120.38 & 120.55 & 120.57 & 120.57 & 120.52 & 119.99 & 94.17 & 61.47 \\
\hline 15.00 & 65.49 & 114.78 & 120.65 & 120.83 & 120.86 & 120.85 & 120.79 & 120.25 & 94.43 & 61.71 \\
\hline 16.00 & 65.99 & 116.29 & 122.26 & 122.45 & 122.48 & 122.48 & 122.41 & 121.86 & 95.50 & 62.07 \\
\hline 17.00 & 66.26 & 116.55 & 122.53 & 122.72 & 122.75 & 122.75 & 122.68 & 122.12 & 95.76 & 62.44 \\
\hline 18.00 & 66.72 & 118.17 & 124.26 & 124.45 & 124.49 & 124.49 & 124.41 & 123.84 & 96.92 & 62.83 \\
\hline 19.00 & 67.01 & 118.45 & 124.54 & 124.74 & 124.78 & 124.77 & 124.69 & 124.12 & 97.21 & 63.07 \\
\hline 20.00 & 67.51 & 120.15 & 126.36 & 126.56 & 126.60 & 126.60 & 126.51 & 125.93 & 98.48 & 63.40 \\
\hline 21.00 & 67.76 & 120.40 & 126.60 & 126.81 & 126.85 & 126.85 & 126.76 & 126.17 & 98.74 & 63.61 \\
\hline 22.00 & 60.61 & 66.04 & 67.89 & 68.08 & 68.14 & 68.13 & 68.01 & 67.49 & 63.23 & 59.39 \\
\hline 22.50 & 58.39 & 58.37 & 58.67 & 58.81 & 58.87 & 58.87 & 58.75 & 58.52 & 58.20 & 57.89 \\
\hline
\end{tabular}


Table B - 7. Temperature $\left({ }^{\circ} \mathrm{C}\right)$ map of the east side of plate $2 \mathrm{BZ}$ at MOC $141 \mathrm{~A}$ (15 EFPD).

\begin{tabular}{|c||c|c|c|c|c|c|c|c|c|c|}
\hline \multirow{2}{*}{$\begin{array}{c}\text { Length of } \\
\text { Plate (in) }\end{array}$} & \multicolumn{10}{|c|}{ Width of Plate (inches) } \\
\hline 22.625 & 58.20 & 58.38 & 58.74 & 58.91 & 58.98 & 58.97 & 58.83 & 58.57 & 58.20 & 57.84 \\
\hline 23.00 & 59.88 & 64.47 & 66.05 & 66.25 & 66.32 & 66.31 & 66.17 & 65.68 & 62.19 & 59.03 \\
\hline 24.00 & 65.60 & 108.79 & 114.03 & 114.26 & 114.33 & 114.33 & 114.20 & 113.63 & 91.14 & 62.65 \\
\hline 25.00 & 65.78 & 108.95 & 114.18 & 114.42 & 114.49 & 114.49 & 114.35 & 113.78 & 91.33 & 62.82 \\
\hline 26.00 & 65.77 & 107.76 & 112.89 & 113.13 & 113.20 & 113.20 & 113.06 & 112.48 & 90.63 & 62.91 \\
\hline 27.00 & 65.95 & 107.98 & 113.11 & 113.36 & 113.43 & 113.43 & 113.28 & 112.70 & 90.84 & 63.08 \\
\hline 28.00 & 66.12 & 108.23 & 113.36 & 113.62 & 113.69 & 113.69 & 113.54 & 112.95 & 91.06 & 63.23 \\
\hline 29.00 & 66.29 & 108.46 & 113.59 & 113.85 & 113.93 & 113.92 & 113.77 & 113.17 & 91.26 & 63.35 \\
\hline 30.00 & 66.32 & 107.77 & 112.84 & 113.10 & 113.18 & 113.18 & 113.01 & 112.42 & 90.88 & 63.42 \\
\hline 31.00 & 66.45 & 107.97 & 113.03 & 113.30 & 113.38 & 113.38 & 113.21 & 112.62 & 91.07 & 63.55 \\
\hline 32.00 & 66.36 & 106.61 & 111.55 & 111.82 & 111.90 & 111.90 & 111.73 & 111.13 & 90.24 & 63.53 \\
\hline 33.00 & 66.48 & 106.82 & 111.75 & 112.02 & 112.11 & 112.10 & 111.93 & 111.34 & 90.43 & 63.62 \\
\hline 34.00 & 66.23 & 104.44 & 109.15 & 109.42 & 109.51 & 109.51 & 109.33 & 108.74 & 88.94 & 63.49 \\
\hline 35.00 & 66.32 & 104.61 & 109.32 & 109.60 & 109.69 & 109.68 & 109.50 & 108.91 & 89.10 & 63.60 \\
\hline 36.00 & 66.02 & 102.06 & 106.54 & 106.82 & 106.91 & 106.90 & 106.72 & 106.14 & 87.50 & 63.45 \\
\hline 37.00 & 66.10 & 102.24 & 106.71 & 106.99 & 107.09 & 107.08 & 106.89 & 106.31 & 87.66 & 63.50 \\
\hline 38.00 & 65.66 & 98.84 & 103.01 & 103.28 & 103.38 & 103.38 & 103.18 & 102.62 & 85.50 & 63.21 \\
\hline 39.00 & 65.70 & 98.98 & 103.14 & 103.42 & 103.51 & 103.51 & 103.32 & 102.75 & 85.62 & 63.24 \\
\hline 40.00 & 65.10 & 94.51 & 98.24 & 98.52 & 98.62 & 98.62 & 98.42 & 97.88 & 82.77 & 62.88 \\
\hline 41.00 & 65.10 & 94.60 & 98.34 & 98.62 & 98.72 & 98.71 & 98.52 & 97.97 & 82.85 & 62.88 \\
\hline 42.00 & 64.32 & 89.13 & 92.28 & 92.56 & 92.66 & 92.65 & 92.45 & 91.94 & 79.31 & 62.37 \\
\hline 43.00 & 64.28 & 89.19 & 92.34 & 92.61 & 92.72 & 92.71 & 92.51 & 92.00 & 79.36 & 62.33 \\
\hline 44.00 & 61.00 & 63.25 & 63.87 & 64.12 & 64.23 & 64.22 & 64.00 & 63.63 & 62.71 & 60.26 \\
\hline 45.00 & 60.44 & 62.69 & 63.13 & 63.36 & 63.47 & 63.47 & 63.25 & 62.95 & 62.26 & 59.80 \\
\hline
\end{tabular}


Table B - 8. Temperature $\left({ }^{\circ} \mathrm{C}\right)$ map of the west side of plate $2 \mathrm{BZ}$ at MOC 141A (15 EFPD).

\begin{tabular}{|c||c|c|c|c|c|c|c|c|c|c|}
\hline \multirow{2}{*}{$\begin{array}{c}\text { Length of } \\
\text { Plate (in) }\end{array}$} & 0.05 & 0.25 & 0.50 & 0.75 & 1.00 & 1.25 & 1.50 & 1.75 & 2.00 & 2.24 \\
\hline 22.625 & 58.48 & 58.42 & 58.73 & 58.87 & 58.94 & 58.93 & 58.81 & 58.57 & 58.25 & 57.98 \\
\hline 23.00 & 60.24 & 64.50 & 66.04 & 66.21 & 66.27 & 66.27 & 66.14 & 65.68 & 62.23 & 59.15 \\
\hline 24.00 & 66.26 & 108.87 & 114.06 & 114.26 & 114.31 & 114.31 & 114.21 & 113.67 & 91.20 & 62.82 \\
\hline 25.00 & 66.40 & 108.99 & 114.17 & 114.38 & 114.43 & 114.43 & 114.32 & 113.78 & 91.36 & 62.98 \\
\hline 26.00 & 66.38 & 107.80 & 112.87 & 113.08 & 113.14 & 113.13 & 113.02 & 112.49 & 90.66 & 63.10 \\
\hline 27.00 & 66.55 & 108.02 & 113.09 & 113.31 & 113.36 & 113.36 & 113.25 & 112.70 & 90.87 & 63.28 \\
\hline 28.00 & 66.72 & 108.27 & 113.35 & 113.56 & 113.62 & 113.62 & 113.50 & 112.95 & 91.09 & 63.40 \\
\hline 29.00 & 66.87 & 108.48 & 113.55 & 113.77 & 113.83 & 113.83 & 113.71 & 113.16 & 91.28 & 63.48 \\
\hline 30.00 & 66.89 & 107.82 & 112.82 & 113.04 & 113.10 & 113.10 & 112.97 & 112.43 & 90.92 & 63.54 \\
\hline 31.00 & 67.01 & 108.01 & 113.01 & 113.24 & 113.30 & 113.30 & 113.17 & 112.62 & 91.10 & 63.68 \\
\hline 32.00 & 66.90 & 106.66 & 111.53 & 111.75 & 111.82 & 111.81 & 111.68 & 111.14 & 90.28 & 63.65 \\
\hline 33.00 & 67.01 & 106.86 & 111.73 & 111.96 & 112.02 & 112.02 & 111.89 & 111.34 & 90.47 & 63.72 \\
\hline 34.00 & 66.72 & 104.48 & 109.13 & 109.36 & 109.43 & 109.42 & 109.29 & 108.75 & 88.98 & 63.59 \\
\hline 35.00 & 66.81 & 104.65 & 109.30 & 109.53 & 109.60 & 109.59 & 109.46 & 108.92 & 89.14 & 63.72 \\
\hline 36.00 & 66.47 & 102.11 & 106.52 & 106.75 & 106.82 & 106.81 & 106.67 & 106.14 & 87.54 & 63.56 \\
\hline 37.00 & 66.53 & 102.26 & 106.67 & 106.91 & 106.98 & 106.97 & 106.83 & 106.30 & 87.68 & 63.60 \\
\hline 38.00 & 66.05 & 98.89 & 102.98 & 103.21 & 103.29 & 103.28 & 103.13 & 102.62 & 85.54 & 63.27 \\
\hline 39.00 & 66.07 & 99.02 & 103.11 & 103.35 & 103.42 & 103.41 & 103.27 & 102.75 & 85.66 & 63.29 \\
\hline 40.00 & 65.42 & 94.55 & 98.22 & 98.45 & 98.53 & 98.52 & 98.37 & 97.88 & 82.81 & 62.92 \\
\hline 41.00 & 65.40 & 94.65 & 98.32 & 98.55 & 98.62 & 98.61 & 98.46 & 97.98 & 82.90 & 62.90 \\
\hline 42.00 & 64.56 & 89.17 & 92.26 & 92.48 & 92.56 & 92.55 & 92.40 & 91.94 & 79.35 & 62.37 \\
\hline 43.00 & 64.51 & 89.23 & 92.32 & 92.54 & 92.62 & 92.61 & 92.45 & 92.01 & 79.40 & 62.32 \\
\hline 44.00 & 61.04 & 63.29 & 63.85 & 64.06 & 64.15 & 64.14 & 63.96 & 63.63 & 62.76 & 60.23 \\
\hline 45.00 & 60.45 & 62.72 & 63.11 & 63.31 & 63.40 & 63.38 & 63.21 & 62.95 & 62.31 & 59.77 \\
\hline
\end{tabular}


Table B - 9. Temperature $\left({ }^{\circ} \mathrm{C}\right)$ map of the east side of plate $2 \mathrm{TT}$ at MOC 141A (25 EFPD).

\begin{tabular}{|c||c|c|c|c|c|c|c|c|c|c|}
\hline \multirow{2}{*}{$\begin{array}{c}\text { Length of } \\
\text { Plate (in) }\end{array}$} & \multicolumn{10}{|c|}{ Width of Plate (inches) } \\
\hline 0.125 & 51.92 & 51.77 & 51.77 & 51.76 & 51.76 & 51.76 & 51.76 & 51.77 & 51.80 & 51.93 \\
\hline 1.00 & 52.91 & 52.48 & 52.67 & 52.69 & 52.69 & 52.69 & 52.68 & 52.61 & 52.41 & 52.80 \\
\hline 2.00 & 56.73 & 81.80 & 84.84 & 84.92 & 84.92 & 84.92 & 84.91 & 84.64 & 71.26 & 55.27 \\
\hline 3.00 & 57.10 & 82.09 & 85.16 & 85.23 & 85.24 & 85.24 & 85.23 & 84.94 & 71.56 & 55.61 \\
\hline 4.00 & 58.28 & 89.16 & 92.79 & 92.88 & 92.89 & 92.88 & 92.87 & 92.56 & 76.42 & 56.44 \\
\hline 5.00 & 58.56 & 89.36 & 93.00 & 93.09 & 93.10 & 93.10 & 93.08 & 92.75 & 76.63 & 56.70 \\
\hline 6.00 & 59.72 & 95.64 & 99.76 & 99.85 & 99.86 & 99.86 & 99.84 & 99.50 & 81.03 & 57.51 \\
\hline 7.00 & 60.02 & 95.86 & 100.00 & 100.11 & 100.13 & 100.13 & 100.10 & 99.72 & 81.27 & 57.79 \\
\hline 8.00 & 60.97 & 100.63 & 105.44 & 105.57 & 105.59 & 105.59 & 105.55 & 105.12 & 84.62 & 58.47 \\
\hline 9.00 & 61.23 & 100.85 & 105.68 & 105.81 & 105.83 & 105.83 & 105.79 & 105.35 & 84.85 & 58.77 \\
\hline 10.00 & 62.15 & 105.44 & 110.64 & 110.79 & 110.81 & 110.81 & 110.76 & 110.29 & 87.92 & 59.42 \\
\hline 11.00 & 62.42 & 105.68 & 110.89 & 111.04 & 111.06 & 111.06 & 111.01 & 110.53 & 88.15 & 59.65 \\
\hline 12.00 & 63.14 & 108.74 & 114.19 & 114.35 & 114.38 & 114.38 & 114.32 & 113.82 & 90.23 & 60.21 \\
\hline 13.00 & 63.41 & 108.98 & 114.44 & 114.61 & 114.64 & 114.64 & 114.58 & 114.06 & 90.47 & 60.48 \\
\hline 14.00 & 64.04 & 111.76 & 117.44 & 117.62 & 117.65 & 117.65 & 117.58 & 117.05 & 92.36 & 60.96 \\
\hline 15.00 & 64.32 & 112.02 & 117.70 & 117.88 & 117.92 & 117.92 & 117.84 & 117.30 & 92.60 & 61.21 \\
\hline 16.00 & 64.77 & 113.40 & 119.19 & 119.38 & 119.42 & 119.42 & 119.33 & 118.78 & 93.59 & 61.56 \\
\hline 17.00 & 65.03 & 113.65 & 119.44 & 119.64 & 119.68 & 119.68 & 119.59 & 119.03 & 93.84 & 61.86 \\
\hline 18.00 & 65.46 & 115.17 & 121.06 & 121.27 & 121.31 & 121.31 & 121.22 & 120.64 & 94.90 & 62.22 \\
\hline 19.00 & 65.73 & 115.43 & 121.33 & 121.54 & 121.59 & 121.59 & 121.49 & 120.91 & 95.15 & 62.46 \\
\hline 20.00 & 66.20 & 117.13 & 123.14 & 123.36 & 123.41 & 123.41 & 123.31 & 122.71 & 96.35 & 62.81 \\
\hline 21.00 & 66.44 & 117.36 & 123.38 & 123.60 & 123.66 & 123.66 & 123.54 & 122.94 & 96.59 & 63.03 \\
\hline 22.00 & 59.97 & 65.40 & 67.21 & 67.41 & 67.47 & 67.47 & 67.33 & 66.80 & 62.71 & 59.06 \\
\hline 22.50 & 57.89 & 58.07 & 58.40 & 58.56 & 58.62 & 58.62 & 58.48 & 58.24 & 57.92 & 57.57 \\
\hline
\end{tabular}


Table B - 10. Temperature $\left({ }^{\circ} \mathrm{C}\right)$ map of the west side of plate 2TT at MOC 141A (25 EFPD).

\begin{tabular}{|c||c|c|c|c|c|c|c|c|c|c|}
\hline \multirow{2}{*}{$\begin{array}{c}\text { Length of } \\
\text { Plate (in) }\end{array}$} & \multicolumn{10}{|c|}{ Width of Plate (inches) } \\
\hline & 0.05 & 0.25 & 0.50 & 0.75 & 1.00 & 1.25 & 1.50 & 1.75 & 2.00 & 2.24 \\
\hline 0.125 & 51.96 & 51.77 & 51.77 & 51.76 & 51.76 & 51.76 & 51.76 & 51.77 & 51.80 & 51.95 \\
\hline 1.00 & 53.04 & 52.48 & 52.67 & 52.69 & 52.69 & 52.69 & 52.68 & 52.61 & 52.41 & 52.87 \\
\hline 2.00 & 57.05 & 81.80 & 84.84 & 84.91 & 84.92 & 84.92 & 84.91 & 84.64 & 71.25 & 55.36 \\
\hline 3.00 & 57.44 & 82.09 & 85.16 & 85.23 & 85.24 & 85.24 & 85.22 & 84.94 & 71.55 & 55.71 \\
\hline 4.00 & 58.69 & 89.16 & 92.79 & 92.87 & 92.88 & 92.88 & 92.87 & 92.56 & 76.40 & 56.56 \\
\hline 5.00 & 58.98 & 89.36 & 92.99 & 93.08 & 93.09 & 93.09 & 93.07 & 92.75 & 76.62 & 56.82 \\
\hline 6.00 & 60.19 & 95.64 & 99.75 & 99.85 & 99.86 & 99.86 & 99.84 & 99.50 & 81.02 & 57.63 \\
\hline 7.00 & 60.47 & 95.84 & 99.95 & 100.06 & 100.08 & 100.08 & 100.05 & 99.69 & 81.23 & 57.87 \\
\hline 8.00 & 61.51 & 100.64 & 105.43 & 105.56 & 105.57 & 105.57 & 105.54 & 105.12 & 84.61 & 58.59 \\
\hline 9.00 & 61.78 & 100.86 & 105.67 & 105.80 & 105.82 & 105.81 & 105.78 & 105.35 & 84.84 & 58.96 \\
\hline 10.00 & 62.74 & 105.45 & 110.63 & 110.77 & 110.79 & 110.79 & 110.75 & 110.29 & 87.92 & 59.61 \\
\hline 11.00 & 63.02 & 105.69 & 110.88 & 111.02 & 111.04 & 111.04 & 111.00 & 110.53 & 88.14 & 59.82 \\
\hline 12.00 & 63.77 & 108.75 & 114.18 & 114.33 & 114.35 & 114.35 & 114.31 & 113.82 & 90.23 & 60.37 \\
\hline 13.00 & 64.04 & 109.00 & 114.43 & 114.59 & 114.62 & 114.61 & 114.56 & 114.06 & 90.47 & 60.65 \\
\hline 14.00 & 64.70 & 111.78 & 117.43 & 117.59 & 117.62 & 117.62 & 117.56 & 117.05 & 92.36 & 61.14 \\
\hline 15.00 & 64.99 & 112.04 & 117.69 & 117.86 & 117.89 & 117.89 & 117.83 & 117.30 & 92.61 & 61.37 \\
\hline 16.00 & 65.45 & 113.43 & 119.17 & 119.35 & 119.38 & 119.38 & 119.31 & 118.78 & 93.60 & 61.71 \\
\hline 17.00 & 65.72 & 113.68 & 119.43 & 119.61 & 119.64 & 119.64 & 119.57 & 119.03 & 93.85 & 62.07 \\
\hline 18.00 & 66.15 & 115.20 & 121.05 & 121.24 & 121.27 & 121.27 & 121.20 & 120.65 & 94.92 & 62.45 \\
\hline 19.00 & 66.43 & 115.46 & 121.32 & 121.51 & 121.55 & 121.54 & 121.47 & 120.91 & 95.16 & 62.68 \\
\hline 20.00 & 66.91 & 117.16 & 123.13 & 123.33 & 123.37 & 123.36 & 123.28 & 122.71 & 96.37 & 62.99 \\
\hline 21.00 & 67.15 & 117.39 & 123.36 & 123.57 & 123.61 & 123.60 & 123.52 & 122.95 & 96.62 & 63.20 \\
\hline 22.00 & 60.35 & 65.43 & 67.20 & 67.38 & 67.43 & 67.42 & 67.31 & 66.81 & 62.75 & 59.18 \\
\hline 22.50 & 58.23 & 58.11 & 58.39 & 58.52 & 58.58 & 58.57 & 58.46 & 58.24 & 57.96 & 57.75 \\
\hline
\end{tabular}


Table B - 11. Temperature $\left({ }^{\circ} \mathrm{C}\right)$ map of the east side of plate 2BZ at MOC 141A (25 EFPD).

\begin{tabular}{|c||c|c|c|c|c|c|c|c|c|c|}
\hline \multirow{2}{*}{$\begin{array}{c}\text { Length of } \\
\text { Plate (in) }\end{array}$} & \multicolumn{10}{|c|}{ Width of Plate (inches) } \\
\hline 22.625 & 58.04 & 58.12 & 58.46 & 58.61 & 58.68 & 58.68 & 58.54 & 58.29 & 57.96 & 57.70 \\
\hline 23.00 & 59.62 & 63.87 & 65.36 & 65.56 & 65.62 & 65.62 & 65.48 & 65.01 & 61.72 & 58.83 \\
\hline 24.00 & 65.02 & 105.82 & 110.82 & 111.04 & 111.11 & 111.10 & 110.98 & 110.43 & 89.15 & 62.24 \\
\hline 25.00 & 65.20 & 106.01 & 111.00 & 111.23 & 111.29 & 111.29 & 111.16 & 110.60 & 89.35 & 62.41 \\
\hline 26.00 & 65.24 & 105.14 & 110.05 & 110.29 & 110.36 & 110.36 & 110.22 & 109.66 & 88.86 & 62.52 \\
\hline 27.00 & 65.40 & 105.35 & 110.27 & 110.51 & 110.58 & 110.57 & 110.43 & 109.87 & 89.06 & 62.69 \\
\hline 28.00 & 65.55 & 105.44 & 110.34 & 110.59 & 110.66 & 110.66 & 110.51 & 109.94 & 89.17 & 62.81 \\
\hline 29.00 & 65.70 & 105.63 & 110.54 & 110.79 & 110.87 & 110.86 & 110.71 & 110.14 & 89.35 & 62.92 \\
\hline 30.00 & 65.78 & 105.31 & 110.17 & 110.42 & 110.50 & 110.49 & 110.34 & 109.77 & 89.20 & 63.02 \\
\hline 31.00 & 65.90 & 105.49 & 110.36 & 110.61 & 110.69 & 110.69 & 110.53 & 109.96 & 89.38 & 63.15 \\
\hline 32.00 & 65.82 & 104.19 & 108.92 & 109.18 & 109.26 & 109.26 & 109.10 & 108.52 & 88.58 & 63.13 \\
\hline 33.00 & 65.94 & 104.36 & 109.10 & 109.36 & 109.45 & 109.44 & 109.28 & 108.70 & 88.74 & 63.22 \\
\hline 34.00 & 65.70 & 102.21 & 106.75 & 107.01 & 107.10 & 107.09 & 106.92 & 106.36 & 87.40 & 63.10 \\
\hline 35.00 & 65.80 & 102.38 & 106.91 & 107.18 & 107.27 & 107.26 & 107.09 & 106.52 & 87.56 & 63.21 \\
\hline 36.00 & 65.52 & 100.09 & 104.41 & 104.68 & 104.76 & 104.76 & 104.58 & 104.02 & 86.12 & 63.07 \\
\hline 37.00 & 65.60 & 100.24 & 104.56 & 104.83 & 104.92 & 104.92 & 104.74 & 104.18 & 86.26 & 63.12 \\
\hline 38.00 & 65.19 & 97.10 & 101.12 & 101.39 & 101.48 & 101.48 & 101.29 & 100.75 & 84.26 & 62.85 \\
\hline 39.00 & 65.23 & 97.23 & 101.25 & 101.52 & 101.61 & 101.61 & 101.42 & 100.88 & 84.38 & 62.88 \\
\hline 40.00 & 64.68 & 93.14 & 96.77 & 97.04 & 97.14 & 97.13 & 96.94 & 96.42 & 81.77 & 62.55 \\
\hline 41.00 & 64.68 & 93.24 & 96.87 & 97.14 & 97.23 & 97.23 & 97.04 & 96.51 & 81.85 & 62.55 \\
\hline 42.00 & 63.94 & 88.13 & 91.14 & 91.41 & 91.51 & 91.50 & 91.30 & 90.82 & 78.50 & 62.07 \\
\hline 43.00 & 63.91 & 88.19 & 91.20 & 91.46 & 91.56 & 91.56 & 91.36 & 90.88 & 78.56 & 62.02 \\
\hline 44.00 & 60.70 & 62.78 & 63.36 & 63.60 & 63.71 & 63.70 & 63.49 & 63.14 & 62.26 & 60.00 \\
\hline 45.00 & 60.14 & 62.22 & 62.64 & 62.86 & 62.97 & 62.96 & 62.75 & 62.47 & 61.82 & 59.55 \\
\hline
\end{tabular}


Table B - 12. Temperature $\left({ }^{\circ} \mathrm{C}\right)$ map of the west side of plate $2 \mathrm{BZ}$ at MOC 141A (25 EFPD).

\begin{tabular}{|c||c|c|c|c|c|c|c|c|c|c|}
\hline \multirow{2}{*}{$\begin{array}{c}\text { Length of } \\
\text { Plate (in) }\end{array}$} & \multicolumn{10}{|c|}{ Width of Plate (inches) } \\
\hline 22.625 & 58.32 & 58.15 & 58.44 & 58.58 & 58.64 & 58.63 & 58.51 & 58.29 & 58.01 & 57.84 \\
\hline 23.00 & 59.98 & 63.90 & 65.35 & 65.52 & 65.58 & 65.57 & 65.45 & 65.02 & 61.77 & 58.94 \\
\hline 24.00 & 65.64 & 105.86 & 110.80 & 111.00 & 111.05 & 111.04 & 110.94 & 110.43 & 89.18 & 62.39 \\
\hline 25.00 & 65.81 & 106.04 & 110.98 & 111.18 & 111.23 & 111.23 & 111.12 & 110.61 & 89.38 & 62.57 \\
\hline 26.00 & 65.83 & 105.18 & 110.04 & 110.24 & 110.29 & 110.29 & 110.18 & 109.66 & 88.89 & 62.71 \\
\hline 27.00 & 65.99 & 105.39 & 110.25 & 110.45 & 110.51 & 110.50 & 110.39 & 109.87 & 89.09 & 62.88 \\
\hline 28.00 & 66.13 & 105.47 & 110.32 & 110.53 & 110.59 & 110.58 & 110.47 & 109.95 & 89.20 & 62.99 \\
\hline 29.00 & 66.28 & 105.69 & 110.54 & 110.75 & 110.81 & 110.81 & 110.69 & 110.16 & 89.40 & 63.08 \\
\hline 30.00 & 66.34 & 105.35 & 110.15 & 110.36 & 110.42 & 110.42 & 110.30 & 109.77 & 89.23 & 63.15 \\
\hline 31.00 & 66.46 & 105.53 & 110.34 & 110.55 & 110.61 & 110.61 & 110.49 & 109.96 & 89.41 & 63.28 \\
\hline 32.00 & 66.35 & 104.23 & 108.90 & 109.12 & 109.18 & 109.17 & 109.05 & 108.52 & 88.61 & 63.25 \\
\hline 33.00 & 66.45 & 104.40 & 109.08 & 109.30 & 109.36 & 109.35 & 109.23 & 108.70 & 88.78 & 63.33 \\
\hline 34.00 & 66.19 & 102.25 & 106.73 & 106.95 & 107.01 & 107.00 & 106.87 & 106.36 & 87.44 & 63.20 \\
\hline 35.00 & 66.27 & 102.42 & 106.89 & 107.11 & 107.18 & 107.17 & 107.04 & 106.52 & 87.59 & 63.33 \\
\hline 36.00 & 65.96 & 100.13 & 104.39 & 104.61 & 104.67 & 104.67 & 104.53 & 104.02 & 86.16 & 63.19 \\
\hline 37.00 & 66.02 & 100.30 & 104.55 & 104.78 & 104.84 & 104.84 & 104.70 & 104.19 & 86.31 & 63.22 \\
\hline 38.00 & 65.57 & 97.14 & 101.10 & 101.32 & 101.39 & 101.38 & 101.24 & 100.75 & 84.30 & 62.92 \\
\hline 39.00 & 65.60 & 97.27 & 101.23 & 101.45 & 101.52 & 101.51 & 101.37 & 100.88 & 84.42 & 62.93 \\
\hline 40.00 & 65.00 & 93.18 & 96.75 & 96.97 & 97.04 & 97.03 & 96.89 & 96.41 & 81.81 & 62.59 \\
\hline 41.00 & 64.98 & 93.28 & 96.84 & 97.06 & 97.13 & 97.12 & 96.98 & 96.51 & 81.89 & 62.58 \\
\hline 42.00 & 64.18 & 88.16 & 91.12 & 91.33 & 91.40 & 91.40 & 91.25 & 90.82 & 78.54 & 62.07 \\
\hline 43.00 & 64.13 & 88.22 & 91.18 & 91.39 & 91.46 & 91.45 & 91.31 & 90.88 & 78.60 & 62.03 \\
\hline 44.00 & 60.73 & 62.81 & 63.34 & 63.54 & 63.63 & 63.62 & 63.45 & 63.14 & 62.31 & 59.98 \\
\hline 45.00 & 60.15 & 62.25 & 62.62 & 62.80 & 62.89 & 62.88 & 62.71 & 62.47 & 61.87 & 59.53 \\
\hline
\end{tabular}


Table B - 13. Temperature $\left({ }^{\circ} \mathrm{C}\right)$ map of the east side of plate 2TT at EOC 141A (32.4 EFPD).

\begin{tabular}{|c||c|c|c|c|c|c|c|c|c|c|}
\hline \multirow{2}{*}{$\begin{array}{c}\text { Length of } \\
\text { Plate (in) }\end{array}$} & \multicolumn{10}{|c|}{ Width of Plate (inches) } \\
\hline & 0.05 & 0.25 & 0.50 & 0.75 & 1.00 & 1.25 & 1.50 & 1.75 & 2.00 & 2.24 \\
\hline 0.125 & 51.92 & 51.77 & 51.76 & 51.76 & 51.76 & 51.76 & 51.76 & 51.77 & 51.80 & 51.93 \\
\hline 1.00 & 52.90 & 52.47 & 52.66 & 52.68 & 52.68 & 52.68 & 52.67 & 52.59 & 52.40 & 52.80 \\
\hline 2.00 & 56.67 & 81.40 & 84.41 & 84.48 & 84.48 & 84.48 & 84.47 & 84.21 & 70.99 & 55.23 \\
\hline 3.00 & 56.93 & 81.61 & 84.63 & 84.70 & 84.71 & 84.71 & 84.69 & 84.42 & 71.21 & 55.48 \\
\hline 4.00 & 58.17 & 88.48 & 92.06 & 92.15 & 92.15 & 92.15 & 92.14 & 91.83 & 75.95 & 56.37 \\
\hline 5.00 & 58.45 & 88.68 & 92.26 & 92.35 & 92.36 & 92.36 & 92.34 & 92.02 & 76.16 & 56.63 \\
\hline 6.00 & 59.56 & 94.70 & 98.74 & 98.84 & 98.85 & 98.85 & 98.83 & 98.49 & 80.37 & 57.40 \\
\hline 7.00 & 59.86 & 94.92 & 98.97 & 99.07 & 99.08 & 99.08 & 99.06 & 98.70 & 80.61 & 57.68 \\
\hline 8.00 & 60.77 & 99.40 & 104.09 & 104.22 & 104.23 & 104.23 & 104.20 & 103.77 & 83.79 & 58.33 \\
\hline 9.00 & 61.02 & 99.60 & 104.32 & 104.45 & 104.47 & 104.47 & 104.43 & 103.99 & 84.02 & 58.63 \\
\hline 10.00 & 61.89 & 103.92 & 108.99 & 109.14 & 109.16 & 109.16 & 109.11 & 108.65 & 86.92 & 59.25 \\
\hline 11.00 & 62.19 & 104.18 & 109.26 & 109.41 & 109.44 & 109.44 & 109.39 & 108.91 & 87.16 & 59.52 \\
\hline 12.00 & 62.83 & 106.91 & 112.21 & 112.37 & 112.39 & 112.39 & 112.34 & 111.84 & 89.02 & 60.00 \\
\hline 13.00 & 63.09 & 107.15 & 112.45 & 112.62 & 112.65 & 112.65 & 112.59 & 112.08 & 89.25 & 60.26 \\
\hline 14.00 & 63.69 & 109.78 & 115.29 & 115.46 & 115.50 & 115.49 & 115.43 & 114.91 & 91.04 & 60.72 \\
\hline 15.00 & 63.96 & 110.02 & 115.54 & 115.72 & 115.76 & 115.76 & 115.68 & 115.15 & 91.28 & 60.96 \\
\hline 16.00 & 64.40 & 111.32 & 116.94 & 117.12 & 117.16 & 117.16 & 117.08 & 116.54 & 92.20 & 61.30 \\
\hline 17.00 & 64.65 & 111.57 & 117.18 & 117.38 & 117.42 & 117.42 & 117.33 & 116.78 & 92.45 & 61.60 \\
\hline 18.00 & 65.04 & 112.90 & 118.61 & 118.81 & 118.85 & 118.85 & 118.76 & 118.20 & 93.39 & 61.94 \\
\hline 19.00 & 65.31 & 113.15 & 118.87 & 119.07 & 119.12 & 119.12 & 119.02 & 118.45 & 93.63 & 62.17 \\
\hline 20.00 & 65.76 & 114.75 & 120.58 & 120.79 & 120.85 & 120.84 & 120.74 & 120.16 & 94.75 & 62.50 \\
\hline 21.00 & 65.99 & 114.98 & 120.81 & 121.03 & 121.08 & 121.08 & 120.97 & 120.38 & 94.96 & 62.71 \\
\hline 22.00 & 59.77 & 64.92 & 66.67 & 66.86 & 66.92 & 66.92 & 66.79 & 66.27 & 62.34 & 58.90 \\
\hline 22.50 & 57.78 & 57.88 & 58.19 & 58.34 & 58.40 & 58.40 & 58.26 & 58.03 & 57.74 & 57.47 \\
\hline
\end{tabular}


Table B - 14. Temperature $\left({ }^{\circ} \mathrm{C}\right)$ map of the west side of plate 2TT at EOC 141A (32.4 EFPD).

\begin{tabular}{|c||c|c|c|c|c|c|c|c|c|c|}
\hline \multirow{2}{*}{$\begin{array}{c}\text { Length of } \\
\text { Plate (in) }\end{array}$} & \multicolumn{10}{|c|}{ Width of Plate (inches) } \\
\hline 0.125 & 51.96 & 51.77 & 51.76 & 51.76 & 51.76 & 51.76 & 51.76 & 51.77 & 51.80 & 51.95 \\
\hline 1.00 & 53.03 & 52.47 & 52.65 & 52.68 & 52.68 & 52.68 & 52.67 & 52.59 & 52.40 & 52.86 \\
\hline 2.00 & 56.99 & 81.40 & 84.41 & 84.48 & 84.48 & 84.48 & 84.47 & 84.21 & 70.98 & 55.32 \\
\hline 3.00 & 57.37 & 81.68 & 84.71 & 84.79 & 84.79 & 84.79 & 84.78 & 84.50 & 71.28 & 55.66 \\
\hline 4.00 & 58.57 & 88.48 & 92.06 & 92.14 & 92.15 & 92.15 & 92.13 & 91.83 & 75.94 & 56.48 \\
\hline 5.00 & 58.86 & 88.68 & 92.26 & 92.35 & 92.35 & 92.35 & 92.34 & 92.02 & 76.15 & 56.74 \\
\hline 6.00 & 60.03 & 94.70 & 98.74 & 98.84 & 98.84 & 98.84 & 98.83 & 98.49 & 80.36 & 57.52 \\
\hline 7.00 & 60.34 & 94.92 & 98.96 & 99.06 & 99.07 & 99.07 & 99.05 & 98.70 & 80.60 & 57.79 \\
\hline 8.00 & 61.29 & 99.41 & 104.08 & 104.20 & 104.22 & 104.22 & 104.19 & 103.77 & 83.79 & 58.45 \\
\hline 9.00 & 61.56 & 99.61 & 104.31 & 104.44 & 104.46 & 104.45 & 104.42 & 103.99 & 84.01 & 58.81 \\
\hline 10.00 & 62.47 & 103.93 & 108.98 & 109.12 & 109.14 & 109.14 & 109.10 & 108.65 & 86.91 & 59.43 \\
\hline 11.00 & 62.78 & 104.20 & 109.26 & 109.40 & 109.42 & 109.42 & 109.37 & 108.91 & 87.16 & 59.68 \\
\hline 12.00 & 63.44 & 106.92 & 112.20 & 112.35 & 112.37 & 112.37 & 112.32 & 111.84 & 89.02 & 60.16 \\
\hline 13.00 & 63.71 & 107.16 & 112.44 & 112.60 & 112.62 & 112.62 & 112.57 & 112.08 & 89.26 & 60.43 \\
\hline 14.00 & 64.34 & 109.80 & 115.28 & 115.44 & 115.47 & 115.46 & 115.41 & 114.91 & 91.05 & 60.90 \\
\hline 15.00 & 64.62 & 110.05 & 115.53 & 115.70 & 115.73 & 115.72 & 115.67 & 115.16 & 91.28 & 61.13 \\
\hline 16.00 & 65.06 & 111.35 & 116.92 & 117.10 & 117.13 & 117.12 & 117.06 & 116.54 & 92.21 & 61.45 \\
\hline 17.00 & 65.32 & 111.59 & 117.17 & 117.35 & 117.38 & 117.38 & 117.31 & 116.78 & 92.46 & 61.80 \\
\hline 18.00 & 65.73 & 112.93 & 118.59 & 118.78 & 118.81 & 118.81 & 118.74 & 118.20 & 93.40 & 62.16 \\
\hline 19.00 & 65.99 & 113.18 & 118.85 & 119.04 & 119.08 & 119.07 & 119.00 & 118.45 & 93.64 & 62.38 \\
\hline 20.00 & 66.45 & 114.78 & 120.56 & 120.76 & 120.80 & 120.79 & 120.71 & 120.16 & 94.76 & 62.68 \\
\hline 21.00 & 66.68 & 115.01 & 120.79 & 120.99 & 121.03 & 121.03 & 120.94 & 120.38 & 94.98 & 62.88 \\
\hline 22.00 & 60.15 & 64.95 & 66.66 & 66.83 & 66.88 & 66.87 & 66.76 & 66.28 & 62.38 & 59.02 \\
\hline 22.50 & 58.11 & 57.91 & 58.17 & 58.30 & 58.36 & 58.35 & 58.24 & 58.03 & 57.78 & 57.66 \\
\hline
\end{tabular}


Table B - 15. Temperature $\left({ }^{\circ} \mathrm{C}\right)$ map of the east side of plate 2BZ at EOC 141A (32.4 EFPD).

\begin{tabular}{|c||c|c|c|c|c|c|c|c|c|c|}
\hline \multirow{2}{*}{$\begin{array}{c}\text { Length of } \\
\text { Plate (in) }\end{array}$} & 0.05 & 0.25 & 0.50 & 0.75 & 1.00 & 1.25 & 1.50 & 1.75 & 2.00 & 2.24 \\
\hline 22.625 & 57.91 & 57.92 & 58.24 & 58.39 & 58.46 & 58.45 & 58.32 & 58.08 & 57.78 & 57.60 \\
\hline 23.00 & 59.43 & 63.43 & 64.86 & 65.05 & 65.11 & 65.11 & 64.97 & 64.52 & 61.38 & 58.67 \\
\hline 24.00 & 64.60 & 103.66 & 108.48 & 108.70 & 108.76 & 108.76 & 108.63 & 108.10 & 87.70 & 61.94 \\
\hline 25.00 & 64.78 & 103.85 & 108.66 & 108.89 & 108.95 & 108.95 & 108.82 & 108.28 & 87.90 & 62.11 \\
\hline 26.00 & 64.82 & 103.11 & 107.85 & 108.08 & 108.15 & 108.15 & 108.01 & 107.47 & 87.49 & 62.23 \\
\hline 27.00 & 64.98 & 103.31 & 108.06 & 108.29 & 108.36 & 108.36 & 108.22 & 107.67 & 87.68 & 62.39 \\
\hline 28.00 & 65.12 & 103.35 & 108.09 & 108.32 & 108.39 & 108.39 & 108.25 & 107.70 & 87.76 & 62.51 \\
\hline 29.00 & 65.26 & 103.55 & 108.28 & 108.52 & 108.59 & 108.59 & 108.44 & 107.89 & 87.93 & 62.61 \\
\hline 30.00 & 65.34 & 103.25 & 107.94 & 108.18 & 108.26 & 108.25 & 108.10 & 107.55 & 87.80 & 62.71 \\
\hline 31.00 & 65.46 & 103.43 & 108.12 & 108.37 & 108.45 & 108.44 & 108.29 & 107.73 & 87.97 & 62.83 \\
\hline 32.00 & 65.40 & 102.32 & 106.90 & 107.15 & 107.23 & 107.23 & 107.07 & 106.52 & 87.30 & 62.83 \\
\hline 33.00 & 65.51 & 102.51 & 107.09 & 107.35 & 107.43 & 107.42 & 107.26 & 106.71 & 87.48 & 62.91 \\
\hline 34.00 & 65.30 & 100.45 & 104.84 & 105.10 & 105.18 & 105.18 & 105.01 & 104.46 & 86.19 & 62.80 \\
\hline 35.00 & 65.38 & 100.61 & 105.01 & 105.26 & 105.35 & 105.34 & 105.17 & 104.62 & 86.34 & 62.91 \\
\hline 36.00 & 65.15 & 98.59 & 102.79 & 103.05 & 103.13 & 103.13 & 102.96 & 102.41 & 85.08 & 62.79 \\
\hline 37.00 & 65.22 & 98.74 & 102.94 & 103.20 & 103.28 & 103.28 & 103.11 & 102.56 & 85.21 & 62.84 \\
\hline 38.00 & 64.85 & 95.85 & 99.77 & 100.03 & 100.12 & 100.11 & 99.94 & 99.41 & 83.37 & 62.59 \\
\hline 39.00 & 64.88 & 95.97 & 99.89 & 100.16 & 100.25 & 100.24 & 100.06 & 99.53 & 83.48 & 62.62 \\
\hline 40.00 & 64.36 & 92.10 & 95.65 & 95.91 & 96.01 & 96.00 & 95.82 & 95.31 & 81.01 & 62.30 \\
\hline 41.00 & 64.36 & 92.20 & 95.75 & 96.01 & 96.10 & 96.09 & 95.91 & 95.40 & 81.09 & 62.30 \\
\hline 42.00 & 63.66 & 87.41 & 90.36 & 90.59 & 90.68 & 90.68 & 90.50 & 90.06 & 77.93 & 61.84 \\
\hline 43.00 & 63.63 & 87.47 & 90.41 & 90.65 & 90.74 & 90.73 & 90.56 & 90.12 & 77.98 & 61.80 \\
\hline 44.00 & 60.47 & 62.42 & 62.99 & 63.22 & 63.32 & 63.31 & 63.11 & 62.77 & 61.92 & 59.81 \\
\hline 45.00 & 59.92 & 61.87 & 62.27 & 62.48 & 62.59 & 62.58 & 62.38 & 62.11 & 61.50 & 59.36 \\
\hline
\end{tabular}


Table B - 16. Temperature $\left({ }^{\circ} \mathrm{C}\right)$ map of the west side of plate 2BZ at EOC 141A (32.4 EFPD).

\begin{tabular}{|c||c|c|c|c|c|c|c|c|c|c|}
\hline \multirow{2}{*}{$\begin{array}{c}\text { Length of } \\
\text { Plate (in) }\end{array}$} & \multicolumn{10}{|c|}{ Width of Plate (inches) } \\
\hline 22.625 & 58.20 & 57.95 & 58.23 & 58.36 & 58.41 & 58.41 & 58.30 & 58.08 & 57.82 & 57.74 \\
\hline 23.00 & 59.78 & 63.46 & 64.85 & 65.01 & 65.07 & 65.06 & 64.95 & 64.53 & 61.42 & 58.79 \\
\hline 24.00 & 65.24 & 103.73 & 108.50 & 108.69 & 108.74 & 108.74 & 108.64 & 108.14 & 87.76 & 62.11 \\
\hline 25.00 & 65.38 & 103.89 & 108.64 & 108.84 & 108.89 & 108.88 & 108.78 & 108.28 & 87.92 & 62.27 \\
\hline 26.00 & 65.41 & 103.15 & 107.83 & 108.03 & 108.08 & 108.08 & 107.97 & 107.47 & 87.51 & 62.42 \\
\hline 27.00 & 65.56 & 103.35 & 108.04 & 108.24 & 108.29 & 108.29 & 108.18 & 107.67 & 87.71 & 62.58 \\
\hline 28.00 & 65.69 & 103.39 & 108.07 & 108.27 & 108.32 & 108.32 & 108.21 & 107.70 & 87.79 & 62.68 \\
\hline 29.00 & 65.83 & 103.60 & 108.28 & 108.48 & 108.54 & 108.53 & 108.42 & 107.91 & 87.98 & 62.76 \\
\hline 30.00 & 65.89 & 103.28 & 107.92 & 108.12 & 108.18 & 108.18 & 108.06 & 107.55 & 87.83 & 62.83 \\
\hline 31.00 & 66.00 & 103.46 & 108.10 & 108.31 & 108.37 & 108.36 & 108.24 & 107.73 & 88.00 & 62.96 \\
\hline 32.00 & 65.92 & 102.36 & 106.88 & 107.09 & 107.15 & 107.14 & 107.02 & 106.51 & 87.33 & 62.95 \\
\hline 33.00 & 66.02 & 102.55 & 107.07 & 107.28 & 107.34 & 107.34 & 107.21 & 106.70 & 87.50 & 63.01 \\
\hline 34.00 & 65.77 & 100.49 & 104.82 & 105.03 & 105.09 & 105.09 & 104.96 & 104.46 & 86.22 & 62.90 \\
\hline 35.00 & 65.85 & 100.65 & 104.98 & 105.19 & 105.26 & 105.25 & 105.12 & 104.62 & 86.37 & 63.03 \\
\hline 36.00 & 65.58 & 98.63 & 102.76 & 102.98 & 103.04 & 103.03 & 102.90 & 102.41 & 85.11 & 62.91 \\
\hline 37.00 & 65.64 & 98.78 & 102.91 & 103.13 & 103.19 & 103.18 & 103.05 & 102.56 & 85.24 & 62.94 \\
\hline 38.00 & 65.22 & 95.88 & 99.74 & 99.96 & 100.02 & 100.02 & 99.88 & 99.40 & 83.40 & 62.65 \\
\hline 39.00 & 65.25 & 96.01 & 99.87 & 100.08 & 100.15 & 100.14 & 100.01 & 99.53 & 83.52 & 62.67 \\
\hline 40.00 & 64.68 & 92.14 & 95.63 & 95.84 & 95.91 & 95.90 & 95.76 & 95.30 & 81.04 & 62.35 \\
\hline 41.00 & 64.66 & 92.23 & 95.72 & 95.93 & 96.00 & 95.99 & 95.85 & 95.40 & 81.13 & 62.33 \\
\hline 42.00 & 63.91 & 87.44 & 90.33 & 90.52 & 90.59 & 90.58 & 90.45 & 90.06 & 77.96 & 61.85 \\
\hline 43.00 & 63.85 & 87.50 & 90.39 & 90.57 & 90.64 & 90.63 & 90.50 & 90.11 & 78.01 & 61.81 \\
\hline 44.00 & 60.51 & 62.44 & 62.97 & 63.16 & 63.24 & 63.23 & 63.07 & 62.76 & 61.97 & 59.79 \\
\hline 45.00 & 59.93 & 61.90 & 62.25 & 62.42 & 62.51 & 62.49 & 62.34 & 62.10 & 61.54 & 59.34 \\
\hline
\end{tabular}


Table B - 17. Temperature $\left({ }^{\circ} \mathrm{C}\right)$ map of the east side of plate 2TT at BOC 142A (32.4 EFPD).

\begin{tabular}{|c||c|c|c|c|c|c|c|c|c|c|}
\hline \multirow{2}{*}{$\begin{array}{c}\text { Length of } \\
\text { Plate (in) }\end{array}$} & \multicolumn{10}{|c|}{ Width of Plate (inches) } \\
\hline & 0.05 & 0.25 & 0.50 & 0.75 & 1.00 & 1.25 & 1.50 & 1.75 & 2.00 & 2.24 \\
\hline 0.125 & 51.93 & 51.78 & 51.77 & 51.77 & 51.77 & 51.77 & 51.77 & 51.77 & 51.80 & 51.94 \\
\hline 1.00 & 52.89 & 52.43 & 52.60 & 52.61 & 52.62 & 52.62 & 52.61 & 52.54 & 52.37 & 52.80 \\
\hline 2.00 & 56.39 & 79.33 & 82.15 & 82.22 & 82.23 & 82.23 & 82.22 & 81.96 & 69.61 & 55.06 \\
\hline 3.00 & 56.74 & 79.59 & 82.44 & 82.52 & 82.52 & 82.52 & 82.51 & 82.24 & 69.88 & 55.38 \\
\hline 4.00 & 57.78 & 85.73 & 89.09 & 89.17 & 89.18 & 89.18 & 89.16 & 88.86 & 74.07 & 56.12 \\
\hline 5.00 & 58.05 & 85.92 & 89.28 & 89.36 & 89.37 & 89.37 & 89.35 & 89.05 & 74.28 & 56.38 \\
\hline 6.00 & 59.10 & 91.65 & 95.45 & 95.55 & 95.56 & 95.56 & 95.54 & 95.21 & 78.25 & 57.11 \\
\hline 7.00 & 59.40 & 91.86 & 95.67 & 95.77 & 95.78 & 95.78 & 95.76 & 95.42 & 78.48 & 57.39 \\
\hline 8.00 & 60.38 & 96.80 & 101.07 & 101.19 & 101.21 & 101.21 & 101.17 & 100.78 & 81.97 & 58.08 \\
\hline 9.00 & 60.63 & 96.99 & 101.30 & 101.42 & 101.44 & 101.44 & 101.40 & 100.99 & 82.19 & 58.38 \\
\hline 10.00 & 61.38 & 100.43 & 105.20 & 105.33 & 105.35 & 105.35 & 105.31 & 104.87 & 84.61 & 58.93 \\
\hline 11.00 & 61.68 & 100.68 & 105.46 & 105.60 & 105.62 & 105.62 & 105.57 & 105.12 & 84.85 & 59.19 \\
\hline 12.00 & 62.44 & 104.28 & 109.35 & 109.50 & 109.53 & 109.53 & 109.47 & 109.00 & 87.28 & 59.76 \\
\hline 13.00 & 62.70 & 104.51 & 109.59 & 109.75 & 109.78 & 109.78 & 109.72 & 109.24 & 87.51 & 60.02 \\
\hline 14.00 & 63.24 & 106.75 & 112.01 & 112.18 & 112.21 & 112.21 & 112.14 & 111.64 & 89.04 & 60.45 \\
\hline 15.00 & 63.51 & 106.99 & 112.26 & 112.43 & 112.46 & 112.46 & 112.39 & 111.88 & 89.27 & 60.68 \\
\hline 16.00 & 64.12 & 109.49 & 114.94 & 115.13 & 115.16 & 115.16 & 115.08 & 114.56 & 90.97 & 61.13 \\
\hline 17.00 & 64.38 & 109.73 & 115.19 & 115.38 & 115.42 & 115.42 & 115.34 & 114.80 & 91.22 & 61.44 \\
\hline 18.00 & 64.72 & 110.72 & 116.25 & 116.44 & 116.48 & 116.48 & 116.40 & 115.85 & 91.94 & 61.75 \\
\hline 19.00 & 64.97 & 110.96 & 116.50 & 116.70 & 116.74 & 116.74 & 116.65 & 116.09 & 92.17 & 61.97 \\
\hline 20.00 & 65.39 & 112.32 & 117.95 & 118.16 & 118.20 & 118.20 & 118.10 & 117.54 & 93.13 & 62.28 \\
\hline 21.00 & 65.61 & 112.54 & 118.17 & 118.38 & 118.43 & 118.43 & 118.32 & 117.75 & 93.33 & 62.49 \\
\hline 22.00 & 59.65 & 64.38 & 66.04 & 66.23 & 66.29 & 66.28 & 66.16 & 65.67 & 61.93 & 58.83 \\
\hline 22.50 & 57.74 & 57.61 & 57.88 & 58.02 & 58.09 & 58.08 & 57.96 & 57.74 & 57.51 & 57.47 \\
\hline
\end{tabular}


Table B - 18. Temperature $\left({ }^{\circ} \mathrm{C}\right)$ map of the west side of plate 2TT at BOC 142A (32.4 EFPD).

\begin{tabular}{|c||c|c|c|c|c|c|c|c|c|c|}
\hline \multirow{2}{*}{$\begin{array}{c}\text { Length of } \\
\text { Plate (in) }\end{array}$} & \multicolumn{10}{|c|}{ Width of Plate (inches) } \\
\hline & 0.05 & 0.25 & 0.50 & 0.75 & 1.00 & 1.25 & 1.50 & 1.75 & 2.00 & 2.24 \\
\hline 0.125 & 51.97 & 51.78 & 51.77 & 51.77 & 51.77 & 51.77 & 51.77 & 51.77 & 51.80 & 51.97 \\
\hline 1.00 & 53.02 & 52.43 & 52.60 & 52.61 & 52.62 & 52.62 & 52.61 & 52.54 & 52.37 & 52.87 \\
\hline 2.00 & 56.70 & 79.33 & 82.15 & 82.22 & 82.22 & 82.22 & 82.22 & 81.96 & 69.60 & 55.15 \\
\hline 3.00 & 57.07 & 79.59 & 82.44 & 82.51 & 82.52 & 82.52 & 82.51 & 82.24 & 69.87 & 55.48 \\
\hline 4.00 & 58.17 & 85.74 & 89.08 & 89.16 & 89.17 & 89.17 & 89.16 & 88.86 & 74.06 & 56.24 \\
\hline 5.00 & 58.45 & 85.92 & 89.27 & 89.36 & 89.36 & 89.36 & 89.35 & 89.04 & 74.27 & 56.50 \\
\hline 6.00 & 59.56 & 91.65 & 95.45 & 95.54 & 95.55 & 95.55 & 95.53 & 95.21 & 78.24 & 57.24 \\
\hline 7.00 & 59.87 & 91.86 & 95.67 & 95.76 & 95.77 & 95.77 & 95.75 & 95.41 & 78.47 & 57.51 \\
\hline 8.00 & 60.90 & 96.81 & 101.07 & 101.18 & 101.19 & 101.19 & 101.17 & 100.78 & 81.96 & 58.21 \\
\hline 9.00 & 61.16 & 97.00 & 101.29 & 101.41 & 101.42 & 101.42 & 101.39 & 100.99 & 82.18 & 58.57 \\
\hline 10.00 & 61.95 & 100.44 & 105.19 & 105.32 & 105.34 & 105.33 & 105.30 & 104.87 & 84.61 & 59.12 \\
\hline 11.00 & 62.22 & 100.66 & 105.42 & 105.55 & 105.57 & 105.57 & 105.53 & 105.09 & 84.82 & 59.32 \\
\hline 12.00 & 63.05 & 104.29 & 109.34 & 109.48 & 109.50 & 109.50 & 109.46 & 109.00 & 87.27 & 59.92 \\
\hline 13.00 & 63.32 & 104.53 & 109.58 & 109.73 & 109.75 & 109.75 & 109.70 & 109.23 & 87.51 & 60.19 \\
\hline 14.00 & 63.88 & 106.77 & 112.00 & 112.15 & 112.18 & 112.17 & 112.12 & 111.64 & 89.04 & 60.63 \\
\hline 15.00 & 64.16 & 107.01 & 112.24 & 112.40 & 112.43 & 112.42 & 112.37 & 111.88 & 89.27 & 60.85 \\
\hline 16.00 & 64.79 & 109.51 & 114.93 & 115.10 & 115.12 & 115.12 & 115.06 & 114.56 & 90.98 & 61.29 \\
\hline 17.00 & 65.05 & 109.75 & 115.18 & 115.35 & 115.38 & 115.38 & 115.31 & 114.80 & 91.23 & 61.65 \\
\hline 18.00 & 65.41 & 110.74 & 116.23 & 116.41 & 116.44 & 116.44 & 116.37 & 115.85 & 91.95 & 61.98 \\
\hline 19.00 & 65.66 & 110.99 & 116.48 & 116.66 & 116.69 & 116.69 & 116.62 & 116.09 & 92.18 & 62.20 \\
\hline 20.00 & 66.08 & 112.35 & 117.93 & 118.12 & 118.15 & 118.15 & 118.07 & 117.54 & 93.14 & 62.47 \\
\hline 21.00 & 66.31 & 112.57 & 118.15 & 118.34 & 118.38 & 118.37 & 118.29 & 117.75 & 93.35 & 62.66 \\
\hline 22.00 & 60.04 & 64.40 & 66.03 & 66.19 & 66.24 & 66.23 & 66.13 & 65.67 & 61.96 & 58.97 \\
\hline 22.50 & 58.09 & 57.63 & 57.87 & 57.99 & 58.04 & 58.03 & 57.93 & 57.74 & 57.54 & 57.67 \\
\hline
\end{tabular}


Table B - 19. Temperature $\left({ }^{\circ} \mathrm{C}\right)$ map of the east side of plate $2 \mathrm{BZ}$ at BOC $142 \mathrm{~A}$ (32.4 EFPD).

\begin{tabular}{|c||c|c|c|c|c|c|c|c|c|c|}
\hline \multirow{2}{*}{$\begin{array}{c}\text { Length of } \\
\text { Plate (in) }\end{array}$} & \multicolumn{10}{|c|}{ Width of Plate (inches) } \\
\hline 22.625 & 57.87 & 57.65 & 57.93 & 58.08 & 58.14 & 58.13 & 58.01 & 57.79 & 57.55 & 57.59 \\
\hline 23.00 & 59.33 & 62.95 & 64.32 & 64.49 & 64.55 & 64.55 & 64.42 & 63.99 & 61.01 & 58.62 \\
\hline 24.00 & 64.30 & 101.70 & 106.34 & 106.56 & 106.61 & 106.61 & 106.49 & 105.98 & 86.38 & 61.77 \\
\hline 25.00 & 64.49 & 101.92 & 106.56 & 106.78 & 106.84 & 106.84 & 106.71 & 106.19 & 86.59 & 61.95 \\
\hline 26.00 & 64.58 & 101.54 & 106.15 & 106.37 & 106.43 & 106.43 & 106.30 & 105.78 & 86.42 & 62.09 \\
\hline 27.00 & 64.73 & 101.74 & 106.34 & 106.57 & 106.63 & 106.63 & 106.50 & 105.97 & 86.60 & 62.24 \\
\hline 28.00 & 64.79 & 101.27 & 105.82 & 106.05 & 106.12 & 106.11 & 105.98 & 105.45 & 86.35 & 62.31 \\
\hline 29.00 & 64.93 & 101.47 & 106.02 & 106.26 & 106.33 & 106.32 & 106.18 & 105.65 & 86.53 & 62.42 \\
\hline 30.00 & 64.99 & 101.09 & 105.59 & 105.83 & 105.90 & 105.90 & 105.75 & 105.22 & 86.34 & 62.50 \\
\hline 31.00 & 65.11 & 101.26 & 105.77 & 106.01 & 106.08 & 106.08 & 105.93 & 105.39 & 86.50 & 62.62 \\
\hline 32.00 & 65.01 & 99.95 & 104.33 & 104.57 & 104.65 & 104.64 & 104.49 & 103.96 & 85.70 & 62.59 \\
\hline 33.00 & 65.11 & 100.13 & 104.52 & 104.76 & 104.84 & 104.83 & 104.68 & 104.14 & 85.87 & 62.67 \\
\hline 34.00 & 64.97 & 98.59 & 102.83 & 103.07 & 103.15 & 103.15 & 102.99 & 102.46 & 84.91 & 62.61 \\
\hline 35.00 & 65.05 & 98.75 & 102.98 & 103.23 & 103.31 & 103.31 & 103.15 & 102.62 & 85.06 & 62.70 \\
\hline 36.00 & 64.77 & 96.49 & 100.51 & 100.76 & 100.84 & 100.84 & 100.67 & 100.15 & 83.64 & 62.56 \\
\hline 37.00 & 64.83 & 96.65 & 100.67 & 100.92 & 101.00 & 101.00 & 100.83 & 100.31 & 83.78 & 62.60 \\
\hline 38.00 & 64.43 & 93.60 & 97.32 & 97.57 & 97.66 & 97.65 & 97.48 & 96.97 & 81.82 & 62.33 \\
\hline 39.00 & 64.46 & 93.71 & 97.44 & 97.69 & 97.78 & 97.77 & 97.60 & 97.09 & 81.93 & 62.35 \\
\hline 40.00 & 63.94 & 89.98 & 93.25 & 93.49 & 93.58 & 93.58 & 93.40 & 92.92 & 79.49 & 62.03 \\
\hline 41.00 & 63.93 & 90.06 & 93.33 & 93.58 & 93.67 & 93.66 & 93.49 & 93.01 & 79.56 & 62.02 \\
\hline 42.00 & 63.28 & 85.67 & 88.48 & 88.70 & 88.79 & 88.78 & 88.62 & 88.20 & 76.65 & 61.59 \\
\hline 43.00 & 63.24 & 85.72 & 88.53 & 88.75 & 88.84 & 88.83 & 88.67 & 88.25 & 76.70 & 61.55 \\
\hline 44.00 & 60.24 & 61.93 & 62.46 & 62.68 & 62.77 & 62.77 & 62.58 & 62.26 & 61.49 & 59.65 \\
\hline 45.00 & 59.71 & 61.41 & 61.78 & 61.98 & 62.07 & 62.07 & 61.88 & 61.62 & 61.08 & 59.22 \\
\hline
\end{tabular}


Table B - 20. Temperature $\left({ }^{\circ} \mathrm{C}\right)$ map of the west side of plate $2 \mathrm{BZ}$ at BOC 142A (32.4 EFPD).

\begin{tabular}{|c||c|c|c|c|c|c|c|c|c|c|}
\hline \multirow{2}{*}{$\begin{array}{c}\text { Length of } \\
\text { Plate (in) }\end{array}$} & \multicolumn{10}{|c|}{ Width of Plate (inches) } \\
\hline 22.625 & 58.18 & 57.68 & 57.92 & 58.04 & 58.09 & 58.09 & 57.98 & 57.79 & 57.59 & 57.75 \\
\hline 23.00 & 59.70 & 62.98 & 64.30 & 64.45 & 64.51 & 64.50 & 64.39 & 63.99 & 61.05 & 58.75 \\
\hline 24.00 & 64.91 & 101.73 & 106.32 & 106.51 & 106.55 & 106.55 & 106.46 & 105.98 & 86.40 & 61.93 \\
\hline 25.00 & 65.10 & 101.94 & 106.54 & 106.73 & 106.77 & 106.77 & 106.67 & 106.19 & 86.61 & 62.11 \\
\hline 26.00 & 65.18 & 101.57 & 106.13 & 106.32 & 106.36 & 106.36 & 106.26 & 105.78 & 86.44 & 62.29 \\
\hline 27.00 & 65.32 & 101.76 & 106.32 & 106.51 & 106.56 & 106.55 & 106.46 & 105.97 & 86.62 & 62.45 \\
\hline 28.00 & 65.37 & 101.30 & 105.79 & 105.99 & 106.04 & 106.03 & 105.93 & 105.44 & 86.37 & 62.50 \\
\hline 29.00 & 65.50 & 101.50 & 106.00 & 106.19 & 106.24 & 106.24 & 106.13 & 105.64 & 86.56 & 62.57 \\
\hline 30.00 & 65.55 & 101.12 & 105.57 & 105.76 & 105.82 & 105.81 & 105.70 & 105.21 & 86.36 & 62.64 \\
\hline 31.00 & 65.65 & 101.29 & 105.74 & 105.94 & 105.99 & 105.99 & 105.88 & 105.39 & 86.52 & 62.76 \\
\hline 32.00 & 65.54 & 99.98 & 104.30 & 104.50 & 104.56 & 104.55 & 104.44 & 103.95 & 85.72 & 62.72 \\
\hline 33.00 & 65.63 & 100.16 & 104.48 & 104.69 & 104.74 & 104.74 & 104.62 & 104.13 & 85.89 & 62.78 \\
\hline 34.00 & 65.45 & 98.62 & 102.79 & 103.00 & 103.06 & 103.05 & 102.93 & 102.45 & 84.94 & 62.72 \\
\hline 35.00 & 65.52 & 98.78 & 102.95 & 103.16 & 103.21 & 103.21 & 103.09 & 102.60 & 85.08 & 62.83 \\
\hline 36.00 & 65.21 & 96.52 & 100.48 & 100.68 & 100.74 & 100.73 & 100.61 & 100.14 & 83.66 & 62.69 \\
\hline 37.00 & 65.26 & 96.66 & 100.62 & 100.82 & 100.88 & 100.88 & 100.76 & 100.28 & 83.79 & 62.71 \\
\hline 38.00 & 64.81 & 93.62 & 97.29 & 97.49 & 97.55 & 97.54 & 97.42 & 96.96 & 81.85 & 62.40 \\
\hline 39.00 & 64.83 & 93.74 & 97.40 & 97.61 & 97.67 & 97.66 & 97.54 & 97.08 & 81.95 & 62.41 \\
\hline 40.00 & 64.26 & 90.00 & 93.21 & 93.41 & 93.47 & 93.47 & 93.34 & 92.91 & 79.51 & 62.08 \\
\hline 41.00 & 64.23 & 90.08 & 93.30 & 93.50 & 93.56 & 93.55 & 93.42 & 92.99 & 79.59 & 62.06 \\
\hline 42.00 & 63.53 & 85.69 & 88.45 & 88.62 & 88.68 & 88.68 & 88.56 & 88.18 & 76.67 & 61.61 \\
\hline 43.00 & 63.47 & 85.74 & 88.50 & 88.67 & 88.73 & 88.73 & 88.61 & 88.23 & 76.72 & 61.56 \\
\hline 44.00 & 60.29 & 61.95 & 62.43 & 62.61 & 62.69 & 62.68 & 62.52 & 62.24 & 61.52 & 59.64 \\
\hline 45.00 & 59.74 & 61.43 & 61.75 & 61.91 & 61.99 & 61.98 & 61.83 & 61.61 & 61.11 & 59.21 \\
\hline
\end{tabular}


Table B - 21. Temperature $\left({ }^{\circ} \mathrm{C}\right)$ map of the east side of plate $2 \mathrm{TT}$ at MOC 142A (48.4 EFPD).

\begin{tabular}{|c||c|c|c|c|c|c|c|c|c|c|}
\hline \multirow{2}{*}{$\begin{array}{c}\text { Length of } \\
\text { Plate (in) }\end{array}$} & 0.05 & 0.25 & 0.50 & 0.75 & 1.00 & 1.25 & 1.50 & 1.75 & 2.00 & 2.24 \\
\hline 0.125 & 51.93 & 51.78 & 51.77 & 51.77 & 51.77 & 51.77 & 51.77 & 51.77 & 51.80 & 51.94 \\
\hline 1.00 & 52.94 & 52.49 & 52.67 & 52.69 & 52.69 & 52.69 & 52.68 & 52.61 & 52.42 & 52.84 \\
\hline 2.00 & 56.74 & 81.63 & 84.65 & 84.73 & 84.73 & 84.73 & 84.72 & 84.45 & 71.16 & 55.30 \\
\hline 3.00 & 57.10 & 81.90 & 84.95 & 85.03 & 85.03 & 85.03 & 85.02 & 84.74 & 71.44 & 55.62 \\
\hline 4.00 & 58.14 & 87.95 & 91.49 & 91.57 & 91.58 & 91.58 & 91.56 & 91.26 & 75.60 & 56.37 \\
\hline 5.00 & 58.42 & 88.14 & 91.68 & 91.77 & 91.78 & 91.78 & 91.76 & 91.44 & 75.81 & 56.62 \\
\hline 6.00 & 59.45 & 93.66 & 97.62 & 97.72 & 97.73 & 97.73 & 97.71 & 97.37 & 79.66 & 57.35 \\
\hline 7.00 & 59.75 & 93.87 & 97.84 & 97.94 & 97.95 & 97.95 & 97.93 & 97.58 & 79.89 & 57.63 \\
\hline 8.00 & 60.61 & 98.00 & 102.46 & 102.58 & 102.60 & 102.59 & 102.56 & 102.15 & 82.82 & 58.24 \\
\hline 9.00 & 60.86 & 98.18 & 102.68 & 102.81 & 102.83 & 102.83 & 102.79 & 102.37 & 83.04 & 58.55 \\
\hline 10.00 & 61.62 & 101.69 & 106.56 & 106.70 & 106.72 & 106.72 & 106.68 & 106.23 & 85.46 & 59.10 \\
\hline 11.00 & 61.91 & 101.94 & 106.82 & 106.97 & 106.99 & 106.99 & 106.94 & 106.48 & 85.70 & 59.36 \\
\hline 12.00 & 62.46 & 104.11 & 109.16 & 109.32 & 109.34 & 109.34 & 109.29 & 108.81 & 87.18 & 59.78 \\
\hline 13.00 & 62.71 & 104.33 & 109.40 & 109.56 & 109.58 & 109.58 & 109.52 & 109.04 & 87.41 & 60.04 \\
\hline 14.00 & 63.16 & 105.94 & 111.13 & 111.29 & 111.33 & 111.32 & 111.26 & 110.76 & 88.53 & 60.41 \\
\hline 15.00 & 63.42 & 106.17 & 111.36 & 111.54 & 111.57 & 111.57 & 111.50 & 110.99 & 88.75 & 60.63 \\
\hline 16.00 & 63.95 & 108.16 & 113.50 & 113.68 & 113.72 & 113.72 & 113.64 & 113.12 & 90.12 & 61.03 \\
\hline 17.00 & 64.20 & 108.39 & 113.74 & 113.93 & 113.97 & 113.97 & 113.88 & 113.35 & 90.36 & 61.33 \\
\hline 18.00 & 64.56 & 109.53 & 114.96 & 115.15 & 115.19 & 115.19 & 115.10 & 114.56 & 91.18 & 61.65 \\
\hline 19.00 & 64.82 & 109.77 & 115.20 & 115.40 & 115.45 & 115.45 & 115.35 & 114.80 & 91.40 & 61.88 \\
\hline 20.00 & 65.10 & 110.22 & 115.67 & 115.87 & 115.92 & 115.92 & 115.82 & 115.26 & 91.76 & 62.10 \\
\hline 21.00 & 65.30 & 110.42 & 115.87 & 116.08 & 116.13 & 116.13 & 116.02 & 115.46 & 91.96 & 62.28 \\
\hline 22.00 & 59.58 & 64.11 & 65.72 & 65.90 & 65.97 & 65.96 & 65.83 & 65.36 & 61.76 & 58.78 \\
\hline 22.50 & 57.75 & 57.62 & 57.89 & 58.03 & 58.10 & 58.09 & 57.97 & 57.75 & 57.52 & 57.48 \\
\hline
\end{tabular}


Table B - 22. Temperature $\left({ }^{\circ} \mathrm{C}\right)$ map of the west side of plate 2TT at MOC 142A (48.4 EFPD).

\begin{tabular}{|c||c|c|c|c|c|c|c|c|c|c|}
\hline \multirow{2}{*}{$\begin{array}{c}\text { Length of } \\
\text { Plate (in) }\end{array}$} & \multicolumn{10}{|c|}{ Width of Plate (inches) } \\
\hline 0.125 & 51.97 & 51.78 & 51.77 & 51.77 & 51.77 & 51.77 & 51.77 & 51.77 & 51.80 & 51.97 \\
\hline 1.00 & 53.07 & 52.49 & 52.67 & 52.69 & 52.69 & 52.69 & 52.68 & 52.61 & 52.41 & 52.91 \\
\hline 2.00 & 57.07 & 81.63 & 84.65 & 84.72 & 84.73 & 84.73 & 84.72 & 84.45 & 71.14 & 55.39 \\
\hline 3.00 & 57.45 & 81.90 & 84.95 & 85.03 & 85.03 & 85.03 & 85.02 & 84.74 & 71.43 & 55.72 \\
\hline 4.00 & 58.55 & 87.96 & 91.48 & 91.57 & 91.57 & 91.57 & 91.56 & 91.25 & 75.59 & 56.48 \\
\hline 5.00 & 58.84 & 88.15 & 91.68 & 91.77 & 91.77 & 91.77 & 91.76 & 91.44 & 75.80 & 56.75 \\
\hline 6.00 & 59.92 & 93.66 & 97.62 & 97.71 & 97.72 & 97.72 & 97.70 & 97.37 & 79.65 & 57.48 \\
\hline 7.00 & 60.24 & 93.87 & 97.83 & 97.93 & 97.94 & 97.94 & 97.92 & 97.58 & 79.88 & 57.75 \\
\hline 8.00 & 61.13 & 98.00 & 102.45 & 102.57 & 102.58 & 102.58 & 102.55 & 102.15 & 82.81 & 58.37 \\
\hline 9.00 & 61.40 & 98.19 & 102.67 & 102.79 & 102.81 & 102.81 & 102.78 & 102.36 & 83.04 & 58.74 \\
\hline 10.00 & 62.19 & 101.70 & 106.55 & 106.69 & 106.70 & 106.70 & 106.67 & 106.23 & 85.45 & 59.29 \\
\hline 11.00 & 62.50 & 101.95 & 106.81 & 106.95 & 106.97 & 106.97 & 106.93 & 106.48 & 85.69 & 59.52 \\
\hline 12.00 & 63.07 & 104.12 & 109.15 & 109.30 & 109.32 & 109.32 & 109.27 & 108.81 & 87.18 & 59.95 \\
\hline 13.00 & 63.33 & 104.35 & 109.38 & 109.53 & 109.56 & 109.56 & 109.51 & 109.04 & 87.41 & 60.21 \\
\hline 14.00 & 63.80 & 105.96 & 111.12 & 111.27 & 111.30 & 111.29 & 111.24 & 110.76 & 88.53 & 60.59 \\
\hline 15.00 & 64.06 & 106.19 & 111.35 & 111.51 & 111.54 & 111.53 & 111.48 & 110.99 & 88.75 & 60.80 \\
\hline 16.00 & 64.61 & 108.18 & 113.49 & 113.65 & 113.68 & 113.68 & 113.62 & 113.12 & 90.13 & 61.19 \\
\hline 17.00 & 64.87 & 108.42 & 113.73 & 113.90 & 113.93 & 113.93 & 113.86 & 113.35 & 90.37 & 61.54 \\
\hline 18.00 & 65.24 & 109.56 & 114.94 & 115.12 & 115.15 & 115.15 & 115.08 & 114.56 & 91.19 & 61.89 \\
\hline 19.00 & 65.49 & 109.80 & 115.19 & 115.36 & 115.40 & 115.40 & 115.32 & 114.80 & 91.41 & 62.10 \\
\hline 20.00 & 65.78 & 110.25 & 115.65 & 115.83 & 115.87 & 115.87 & 115.79 & 115.26 & 91.78 & 62.29 \\
\hline 21.00 & 65.98 & 110.45 & 115.85 & 116.04 & 116.08 & 116.07 & 115.99 & 115.46 & 91.97 & 62.46 \\
\hline 22.00 & 59.97 & 64.14 & 65.71 & 65.87 & 65.92 & 65.91 & 65.81 & 65.36 & 61.80 & 58.92 \\
\hline 22.50 & 58.11 & 57.65 & 57.88 & 58.00 & 58.05 & 58.04 & 57.94 & 57.75 & 57.56 & 57.68 \\
\hline
\end{tabular}


Table B - 23. Temperature $\left({ }^{\circ} \mathrm{C}\right)$ map of the east side of plate $2 \mathrm{BZ}$ at MOC 142A (48.4 EFPD).

\begin{tabular}{|c||c|c|c|c|c|c|c|c|c|c|}
\hline \multirow{2}{*}{$\begin{array}{c}\text { Length of } \\
\text { Plate (in) }\end{array}$} & \multicolumn{10}{|c|}{ Width of Plate (inches) } \\
\hline 22.625 & 57.88 & 57.67 & 57.94 & 58.09 & 58.15 & 58.15 & 58.02 & 57.80 & 57.56 & 57.60 \\
\hline 23.00 & 59.29 & 62.78 & 64.11 & 64.28 & 64.34 & 64.34 & 64.21 & 63.79 & 60.91 & 58.59 \\
\hline 24.00 & 64.09 & 100.25 & 104.77 & 104.98 & 105.04 & 105.04 & 104.92 & 104.41 & 85.44 & 61.63 \\
\hline 25.00 & 64.27 & 100.46 & 104.97 & 105.19 & 105.25 & 105.25 & 105.12 & 104.61 & 85.64 & 61.81 \\
\hline 26.00 & 64.35 & 100.00 & 104.46 & 104.68 & 104.75 & 104.75 & 104.62 & 104.10 & 85.41 & 61.94 \\
\hline 27.00 & 64.49 & 100.18 & 104.65 & 104.87 & 104.94 & 104.94 & 104.80 & 104.29 & 85.59 & 62.09 \\
\hline 28.00 & 64.54 & 99.66 & 104.06 & 104.29 & 104.36 & 104.35 & 104.22 & 103.70 & 85.30 & 62.15 \\
\hline 29.00 & 64.68 & 99.86 & 104.26 & 104.49 & 104.56 & 104.56 & 104.42 & 103.90 & 85.48 & 62.26 \\
\hline 30.00 & 64.77 & 99.70 & 104.08 & 104.31 & 104.38 & 104.38 & 104.24 & 103.71 & 85.43 & 62.35 \\
\hline 31.00 & 64.88 & 99.87 & 104.25 & 104.49 & 104.56 & 104.56 & 104.41 & 103.88 & 85.59 & 62.47 \\
\hline 32.00 & 64.82 & 98.85 & 103.13 & 103.37 & 103.44 & 103.44 & 103.29 & 102.77 & 84.98 & 62.46 \\
\hline 33.00 & 64.93 & 99.03 & 103.31 & 103.55 & 103.63 & 103.63 & 103.47 & 102.95 & 85.14 & 62.54 \\
\hline 34.00 & 64.84 & 97.93 & 102.10 & 102.34 & 102.42 & 102.41 & 102.26 & 101.73 & 84.47 & 62.52 \\
\hline 35.00 & 64.92 & 98.08 & 102.25 & 102.50 & 102.58 & 102.57 & 102.41 & 101.89 & 84.61 & 62.61 \\
\hline 36.00 & 64.70 & 96.20 & 100.19 & 100.44 & 100.52 & 100.51 & 100.35 & 99.84 & 83.43 & 62.50 \\
\hline 37.00 & 64.76 & 96.34 & 100.33 & 100.58 & 100.66 & 100.66 & 100.50 & 99.98 & 83.56 & 62.55 \\
\hline 38.00 & 64.37 & 93.40 & 97.10 & 97.35 & 97.44 & 97.43 & 97.26 & 96.76 & 81.68 & 62.28 \\
\hline 39.00 & 64.40 & 93.52 & 97.22 & 97.47 & 97.56 & 97.55 & 97.38 & 96.88 & 81.79 & 62.30 \\
\hline 40.00 & 63.94 & 90.19 & 93.48 & 93.73 & 93.82 & 93.81 & 93.64 & 93.16 & 79.61 & 62.03 \\
\hline 41.00 & 63.93 & 90.27 & 93.57 & 93.82 & 93.91 & 93.90 & 93.73 & 93.25 & 79.69 & 62.02 \\
\hline 42.00 & 63.36 & 86.38 & 89.26 & 89.48 & 89.56 & 89.56 & 89.40 & 88.98 & 77.11 & 61.64 \\
\hline 43.00 & 63.32 & 86.44 & 89.32 & 89.54 & 89.62 & 89.62 & 89.46 & 89.03 & 77.16 & 61.59 \\
\hline 44.00 & 60.22 & 61.90 & 62.43 & 62.64 & 62.74 & 62.73 & 62.54 & 62.22 & 61.45 & 59.64 \\
\hline 45.00 & 59.68 & 61.36 & 61.72 & 61.92 & 62.02 & 62.01 & 61.82 & 61.57 & 61.03 & 59.20 \\
\hline
\end{tabular}


Table B - 24. Temperature $\left({ }^{\circ} \mathrm{C}\right)$ map of the west side of plate $2 \mathrm{BZ}$ at MOC $142 \mathrm{~A}$ (48.4 EFPD).

\begin{tabular}{|c||c|c|c|c|c|c|c|c|c|c|}
\hline \multirow{2}{*}{$\begin{array}{c}\text { Length of } \\
\text { Plate (in) }\end{array}$} & \multicolumn{10}{|c|}{ Width of Plate (inches) } \\
\hline 22.625 & 58.19 & 57.69 & 57.93 & 58.05 & 58.10 & 58.10 & 57.99 & 57.80 & 57.60 & 57.75 \\
\hline 23.00 & 59.66 & 62.81 & 64.09 & 64.24 & 64.30 & 64.29 & 64.18 & 63.79 & 60.94 & 58.72 \\
\hline 24.00 & 64.69 & 100.28 & 104.75 & 104.93 & 104.98 & 104.97 & 104.88 & 104.41 & 85.46 & 61.79 \\
\hline 25.00 & 64.87 & 100.49 & 104.95 & 105.13 & 105.18 & 105.18 & 105.08 & 104.61 & 85.67 & 61.97 \\
\hline 26.00 & 64.93 & 100.03 & 104.44 & 104.63 & 104.68 & 104.67 & 104.57 & 104.10 & 85.43 & 62.14 \\
\hline 27.00 & 65.07 & 100.21 & 104.63 & 104.82 & 104.87 & 104.86 & 104.76 & 104.28 & 85.61 & 62.29 \\
\hline 28.00 & 65.10 & 99.69 & 104.04 & 104.23 & 104.28 & 104.28 & 104.17 & 103.70 & 85.32 & 62.33 \\
\hline 29.00 & 65.24 & 99.88 & 104.24 & 104.43 & 104.48 & 104.48 & 104.37 & 103.89 & 85.50 & 62.41 \\
\hline 30.00 & 65.31 & 99.73 & 104.05 & 104.25 & 104.30 & 104.30 & 104.19 & 103.71 & 85.45 & 62.48 \\
\hline 31.00 & 65.42 & 99.90 & 104.22 & 104.42 & 104.47 & 104.47 & 104.36 & 103.88 & 85.61 & 62.61 \\
\hline 32.00 & 65.34 & 98.88 & 103.10 & 103.30 & 103.35 & 103.35 & 103.24 & 102.76 & 85.00 & 62.59 \\
\hline 33.00 & 65.43 & 99.06 & 103.28 & 103.48 & 103.54 & 103.53 & 103.42 & 102.94 & 85.16 & 62.65 \\
\hline 34.00 & 65.32 & 97.95 & 102.07 & 102.27 & 102.32 & 102.32 & 102.20 & 101.72 & 84.49 & 62.63 \\
\hline 35.00 & 65.39 & 98.11 & 102.22 & 102.42 & 102.48 & 102.48 & 102.36 & 101.88 & 84.64 & 62.74 \\
\hline 36.00 & 65.14 & 96.23 & 100.16 & 100.36 & 100.42 & 100.41 & 100.29 & 99.82 & 83.46 & 62.63 \\
\hline 37.00 & 65.18 & 96.39 & 100.32 & 100.52 & 100.58 & 100.57 & 100.45 & 99.98 & 83.60 & 62.65 \\
\hline 38.00 & 64.75 & 93.42 & 97.07 & 97.27 & 97.33 & 97.33 & 97.20 & 96.75 & 81.70 & 62.36 \\
\hline 39.00 & 64.77 & 93.54 & 97.19 & 97.39 & 97.45 & 97.44 & 97.32 & 96.87 & 81.81 & 62.36 \\
\hline 40.00 & 64.27 & 90.21 & 93.45 & 93.64 & 93.71 & 93.70 & 93.57 & 93.14 & 79.63 & 62.08 \\
\hline 41.00 & 64.24 & 90.30 & 93.54 & 93.74 & 93.80 & 93.79 & 93.66 & 93.24 & 79.71 & 62.06 \\
\hline 42.00 & 63.61 & 86.41 & 89.23 & 89.40 & 89.46 & 89.46 & 89.34 & 88.96 & 77.13 & 61.66 \\
\hline 43.00 & 63.55 & 86.46 & 89.28 & 89.46 & 89.52 & 89.51 & 89.39 & 89.02 & 77.19 & 61.61 \\
\hline 44.00 & 60.28 & 61.91 & 62.40 & 62.58 & 62.65 & 62.64 & 62.49 & 62.21 & 61.48 & 59.63 \\
\hline 45.00 & 59.71 & 61.38 & 61.69 & 61.85 & 61.93 & 61.92 & 61.77 & 61.56 & 61.06 & 59.18 \\
\hline
\end{tabular}


Table B - 25. Temperature $\left({ }^{\circ} \mathrm{C}\right)$ map of the east side of plate $2 \mathrm{TT}$ at MOC 142A (64.4 EFPD).

\begin{tabular}{|c||c|c|c|c|c|c|c|c|c|c|}
\hline \multirow{2}{*}{$\begin{array}{c}\text { Length of } \\
\text { Plate (in) }\end{array}$} & 0.05 & 0.25 & 0.50 & 0.75 & 1.00 & 1.25 & 1.50 & 1.75 & 2.00 & 2.24 \\
\hline 0.125 & 51.93 & 51.78 & 51.77 & 51.77 & 51.77 & 51.77 & 51.77 & 51.77 & 51.80 & 51.94 \\
\hline 1.00 & 52.95 & 52.50 & 52.68 & 52.70 & 52.70 & 52.70 & 52.70 & 52.62 & 52.42 & 52.84 \\
\hline 2.00 & 56.81 & 82.08 & 85.14 & 85.21 & 85.22 & 85.22 & 85.21 & 84.94 & 71.46 & 55.34 \\
\hline 3.00 & 57.17 & 82.35 & 85.44 & 85.52 & 85.52 & 85.52 & 85.51 & 85.23 & 71.75 & 55.67 \\
\hline 4.00 & 58.22 & 88.42 & 91.99 & 92.07 & 92.08 & 92.08 & 92.07 & 91.76 & 75.92 & 56.42 \\
\hline 5.00 & 58.49 & 88.61 & 92.19 & 92.28 & 92.28 & 92.28 & 92.26 & 91.95 & 76.13 & 56.68 \\
\hline 6.00 & 59.51 & 94.00 & 97.99 & 98.08 & 98.09 & 98.09 & 98.07 & 97.73 & 79.89 & 57.39 \\
\hline 7.00 & 59.81 & 94.21 & 98.20 & 98.30 & 98.32 & 98.32 & 98.29 & 97.94 & 80.13 & 57.67 \\
\hline 8.00 & 60.62 & 98.03 & 102.50 & 102.62 & 102.64 & 102.64 & 102.60 & 102.19 & 82.85 & 58.25 \\
\hline 9.00 & 60.87 & 98.22 & 102.72 & 102.85 & 102.87 & 102.87 & 102.83 & 102.40 & 83.07 & 58.56 \\
\hline 10.00 & 61.57 & 101.38 & 106.23 & 106.37 & 106.39 & 106.39 & 106.34 & 105.90 & 85.26 & 59.07 \\
\hline 11.00 & 61.87 & 101.63 & 106.49 & 106.63 & 106.66 & 106.66 & 106.61 & 106.15 & 85.49 & 59.33 \\
\hline 12.00 & 62.43 & 103.86 & 108.90 & 109.05 & 109.08 & 109.08 & 109.02 & 108.55 & 87.03 & 59.76 \\
\hline 13.00 & 62.68 & 104.08 & 109.13 & 109.29 & 109.32 & 109.31 & 109.25 & 108.77 & 87.25 & 60.02 \\
\hline 14.00 & 62.97 & 104.65 & 109.73 & 109.89 & 109.93 & 109.93 & 109.86 & 109.37 & 87.69 & 60.28 \\
\hline 15.00 & 63.22 & 104.87 & 109.95 & 110.13 & 110.16 & 110.16 & 110.09 & 109.59 & 87.90 & 60.50 \\
\hline 16.00 & 63.76 & 106.98 & 112.22 & 112.40 & 112.44 & 112.44 & 112.36 & 111.84 & 89.34 & 60.91 \\
\hline 17.00 & 64.01 & 107.21 & 112.46 & 112.64 & 112.68 & 112.68 & 112.60 & 112.07 & 89.58 & 61.20 \\
\hline 18.00 & 64.32 & 108.00 & 113.29 & 113.48 & 113.53 & 113.53 & 113.44 & 112.90 & 90.17 & 61.49 \\
\hline 19.00 & 64.56 & 108.23 & 113.53 & 113.72 & 113.77 & 113.77 & 113.67 & 113.14 & 90.38 & 61.70 \\
\hline 20.00 & 64.78 & 108.23 & 113.51 & 113.71 & 113.76 & 113.76 & 113.66 & 113.11 & 90.45 & 61.88 \\
\hline 21.00 & 64.96 & 108.41 & 113.70 & 113.90 & 113.96 & 113.95 & 113.85 & 113.30 & 90.64 & 62.06 \\
\hline 22.00 & 59.47 & 63.79 & 65.34 & 65.52 & 65.58 & 65.58 & 65.45 & 64.99 & 61.53 & 58.69 \\
\hline 22.50 & 57.71 & 57.54 & 57.81 & 57.95 & 58.01 & 58.00 & 57.88 & 57.67 & 57.45 & 57.44 \\
\hline
\end{tabular}


Table B - 26. Temperature $\left({ }^{\circ} \mathrm{C}\right)$ map of the west side of plate 2TT at MOC 142A (64.4 EFPD).

\begin{tabular}{|c||c|c|c|c|c|c|c|c|c|c|}
\hline \multirow{2}{*}{$\begin{array}{c}\text { Length of } \\
\text { Plate (in) }\end{array}$} & 0.05 & 0.25 & 0.50 & 0.75 & 1.00 & 1.25 & 1.50 & 1.75 & 2.00 & 2.24 \\
\hline 0.125 & 51.97 & 51.78 & 51.77 & 51.77 & 51.77 & 51.77 & 51.77 & 51.77 & 51.80 & 51.97 \\
\hline 1.00 & 53.08 & 52.50 & 52.68 & 52.70 & 52.70 & 52.70 & 52.70 & 52.62 & 52.42 & 52.92 \\
\hline 2.00 & 57.14 & 82.08 & 85.14 & 85.21 & 85.21 & 85.21 & 85.21 & 84.94 & 71.45 & 55.44 \\
\hline 3.00 & 57.53 & 82.35 & 85.44 & 85.52 & 85.52 & 85.52 & 85.51 & 85.22 & 71.74 & 55.77 \\
\hline 4.00 & 58.63 & 88.42 & 91.99 & 92.07 & 92.08 & 92.08 & 92.06 & 91.75 & 75.91 & 56.54 \\
\hline 5.00 & 58.92 & 88.61 & 92.18 & 92.27 & 92.28 & 92.28 & 92.26 & 91.94 & 76.12 & 56.80 \\
\hline 6.00 & 59.99 & 94.00 & 97.98 & 98.08 & 98.08 & 98.08 & 98.06 & 97.73 & 79.88 & 57.52 \\
\hline 7.00 & 60.30 & 94.21 & 98.20 & 98.30 & 98.31 & 98.30 & 98.28 & 97.94 & 80.12 & 57.79 \\
\hline 8.00 & 61.15 & 98.04 & 102.49 & 102.61 & 102.62 & 102.62 & 102.59 & 102.19 & 82.84 & 58.38 \\
\hline 9.00 & 61.41 & 98.23 & 102.71 & 102.83 & 102.85 & 102.85 & 102.82 & 102.40 & 83.06 & 58.75 \\
\hline 10.00 & 62.15 & 101.39 & 106.22 & 106.35 & 106.37 & 106.37 & 106.33 & 105.89 & 85.25 & 59.26 \\
\hline 11.00 & 62.45 & 101.64 & 106.48 & 106.62 & 106.63 & 106.63 & 106.59 & 106.15 & 85.49 & 59.50 \\
\hline 12.00 & 63.03 & 103.88 & 108.89 & 109.03 & 109.05 & 109.05 & 109.01 & 108.54 & 87.03 & 59.92 \\
\hline 13.00 & 63.29 & 104.10 & 109.12 & 109.26 & 109.29 & 109.29 & 109.24 & 108.77 & 87.25 & 60.19 \\
\hline 14.00 & 63.60 & 104.67 & 109.72 & 109.87 & 109.90 & 109.89 & 109.84 & 109.37 & 87.69 & 60.46 \\
\hline 15.00 & 63.85 & 104.89 & 109.94 & 110.10 & 110.13 & 110.12 & 110.07 & 109.59 & 87.90 & 60.66 \\
\hline 16.00 & 64.41 & 107.00 & 112.21 & 112.37 & 112.40 & 112.40 & 112.34 & 111.84 & 89.35 & 61.06 \\
\hline 17.00 & 64.67 & 107.23 & 112.44 & 112.61 & 112.64 & 112.64 & 112.57 & 112.08 & 89.59 & 61.41 \\
\hline 18.00 & 64.98 & 108.02 & 113.28 & 113.45 & 113.48 & 113.48 & 113.41 & 112.91 & 90.18 & 61.72 \\
\hline 19.00 & 65.23 & 108.25 & 113.51 & 113.69 & 113.72 & 113.72 & 113.65 & 113.14 & 90.40 & 61.92 \\
\hline 20.00 & 65.44 & 108.25 & 113.49 & 113.67 & 113.71 & 113.71 & 113.63 & 113.11 & 90.47 & 62.07 \\
\hline 21.00 & 65.63 & 108.44 & 113.68 & 113.86 & 113.90 & 113.90 & 113.82 & 113.30 & 90.65 & 62.23 \\
\hline 22.00 & 59.85 & 63.81 & 65.33 & 65.49 & 65.54 & 65.53 & 65.43 & 64.99 & 61.56 & 58.83 \\
\hline 22.50 & 58.06 & 57.57 & 57.79 & 57.91 & 57.96 & 57.96 & 57.85 & 57.67 & 57.49 & 57.64 \\
\hline
\end{tabular}


Table B - 27. Temperature $\left({ }^{\circ} \mathrm{C}\right)$ map of the east side of plate $2 \mathrm{BZ}$ at MOC 142A (64.4 EFPD).

\begin{tabular}{|c||c|c|c|c|c|c|c|c|c|c|}
\hline \multirow{2}{*}{$\begin{array}{c}\text { Length of } \\
\text { Plate (in) }\end{array}$} & \multicolumn{10}{|c|}{ Width of Plate (inches) } \\
\hline 22.625 & 57.83 & 57.59 & 57.85 & 57.99 & 58.06 & 58.05 & 57.93 & 57.71 & 57.49 & 57.55 \\
\hline 23.00 & 59.17 & 62.45 & 63.72 & 63.90 & 63.96 & 63.95 & 63.83 & 63.42 & 60.67 & 58.50 \\
\hline 24.00 & 63.74 & 98.20 & 102.54 & 102.74 & 102.80 & 102.80 & 102.68 & 102.19 & 84.08 & 61.40 \\
\hline 25.00 & 63.91 & 98.42 & 102.77 & 102.98 & 103.04 & 103.04 & 102.91 & 102.41 & 84.29 & 61.56 \\
\hline 26.00 & 64.00 & 98.03 & 102.32 & 102.54 & 102.60 & 102.60 & 102.47 & 101.97 & 84.11 & 61.70 \\
\hline 27.00 & 64.14 & 98.21 & 102.50 & 102.72 & 102.78 & 102.78 & 102.65 & 102.15 & 84.28 & 61.84 \\
\hline 28.00 & 64.19 & 97.71 & 101.95 & 102.17 & 102.23 & 102.23 & 102.10 & 101.59 & 84.00 & 61.90 \\
\hline 29.00 & 64.32 & 97.90 & 102.14 & 102.36 & 102.43 & 102.43 & 102.29 & 101.78 & 84.17 & 62.01 \\
\hline 30.00 & 64.41 & 97.76 & 101.98 & 102.21 & 102.28 & 102.27 & 102.13 & 101.62 & 84.13 & 62.10 \\
\hline 31.00 & 64.52 & 97.93 & 102.14 & 102.38 & 102.45 & 102.44 & 102.30 & 101.79 & 84.29 & 62.21 \\
\hline 32.00 & 64.52 & 97.35 & 101.50 & 101.73 & 101.80 & 101.80 & 101.65 & 101.14 & 83.96 & 62.25 \\
\hline 33.00 & 64.62 & 97.53 & 101.68 & 101.91 & 101.99 & 101.98 & 101.83 & 101.32 & 84.12 & 62.32 \\
\hline 34.00 & 64.56 & 96.62 & 100.67 & 100.91 & 100.99 & 100.98 & 100.83 & 100.32 & 83.57 & 62.31 \\
\hline 35.00 & 64.64 & 96.77 & 100.83 & 101.07 & 101.15 & 101.14 & 100.99 & 100.48 & 83.72 & 62.41 \\
\hline 36.00 & 64.49 & 95.40 & 99.32 & 99.56 & 99.64 & 99.64 & 99.48 & 98.98 & 82.87 & 62.35 \\
\hline 37.00 & 64.56 & 95.56 & 99.48 & 99.73 & 99.81 & 99.80 & 99.64 & 99.14 & 83.01 & 62.39 \\
\hline 38.00 & 64.34 & 93.77 & 97.52 & 97.77 & 97.85 & 97.84 & 97.68 & 97.18 & 81.88 & 62.24 \\
\hline 39.00 & 64.37 & 93.90 & 97.65 & 97.90 & 97.98 & 97.97 & 97.81 & 97.31 & 81.99 & 62.26 \\
\hline 40.00 & 63.99 & 91.13 & 94.55 & 94.79 & 94.88 & 94.87 & 94.70 & 94.22 & 80.19 & 62.04 \\
\hline 41.00 & 63.99 & 91.22 & 94.65 & 94.89 & 94.98 & 94.97 & 94.80 & 94.32 & 80.28 & 62.03 \\
\hline 42.00 & 63.41 & 87.29 & 90.25 & 90.47 & 90.55 & 90.55 & 90.39 & 89.97 & 77.67 & 61.65 \\
\hline 43.00 & 63.38 & 87.35 & 90.31 & 90.53 & 90.61 & 90.61 & 90.45 & 90.03 & 77.73 & 61.61 \\
\hline 44.00 & 60.14 & 61.77 & 62.30 & 62.51 & 62.60 & 62.60 & 62.41 & 62.09 & 61.32 & 59.57 \\
\hline 45.00 & 59.58 & 61.21 & 61.56 & 61.76 & 61.85 & 61.84 & 61.66 & 61.41 & 60.88 & 59.11 \\
\hline
\end{tabular}


Table B - 28. Temperature $\left({ }^{\circ} \mathrm{C}\right)$ map of the west side of plate $2 \mathrm{BZ}$ at MOC $142 \mathrm{~A}$ (64.4 EFPD).

\begin{tabular}{|c||c|c|c|c|c|c|c|c|c|c|}
\hline \multirow{2}{*}{$\begin{array}{c}\text { Length of } \\
\text { Plate (in) }\end{array}$} & \multicolumn{10}{|c|}{ Width of Plate (inches) } \\
\hline 22.625 & 58.14 & 57.61 & 57.84 & 57.96 & 58.01 & 58.00 & 57.90 & 57.71 & 57.53 & 57.71 \\
\hline 23.00 & 59.54 & 62.48 & 63.71 & 63.86 & 63.91 & 63.90 & 63.80 & 63.42 & 60.71 & 58.63 \\
\hline 24.00 & 64.33 & 98.23 & 102.51 & 102.69 & 102.74 & 102.73 & 102.64 & 102.19 & 84.10 & 61.55 \\
\hline 25.00 & 64.50 & 98.43 & 102.71 & 102.89 & 102.94 & 102.94 & 102.84 & 102.38 & 84.30 & 61.73 \\
\hline 26.00 & 64.58 & 98.06 & 102.30 & 102.48 & 102.53 & 102.52 & 102.43 & 101.97 & 84.13 & 61.90 \\
\hline 27.00 & 64.70 & 98.24 & 102.48 & 102.66 & 102.71 & 102.71 & 102.61 & 102.14 & 84.30 & 62.05 \\
\hline 28.00 & 64.74 & 97.74 & 101.92 & 102.11 & 102.16 & 102.15 & 102.05 & 101.59 & 84.02 & 62.09 \\
\hline 29.00 & 64.87 & 97.93 & 102.11 & 102.30 & 102.35 & 102.35 & 102.24 & 101.78 & 84.20 & 62.16 \\
\hline 30.00 & 64.94 & 97.79 & 101.95 & 102.14 & 102.19 & 102.19 & 102.08 & 101.61 & 84.16 & 62.23 \\
\hline 31.00 & 65.04 & 97.96 & 102.12 & 102.31 & 102.36 & 102.36 & 102.25 & 101.78 & 84.31 & 62.35 \\
\hline 32.00 & 65.03 & 97.37 & 101.47 & 101.66 & 101.71 & 101.71 & 101.60 & 101.13 & 83.98 & 62.37 \\
\hline 33.00 & 65.12 & 97.55 & 101.65 & 101.84 & 101.90 & 101.89 & 101.78 & 101.31 & 84.14 & 62.43 \\
\hline 34.00 & 65.04 & 96.64 & 100.64 & 100.84 & 100.89 & 100.89 & 100.78 & 100.31 & 83.59 & 62.42 \\
\hline 35.00 & 65.10 & 96.80 & 100.80 & 100.99 & 101.05 & 101.04 & 100.93 & 100.46 & 83.74 & 62.54 \\
\hline 36.00 & 64.93 & 95.43 & 99.29 & 99.49 & 99.54 & 99.54 & 99.42 & 98.96 & 82.89 & 62.48 \\
\hline 37.00 & 64.97 & 95.58 & 99.45 & 99.65 & 99.71 & 99.70 & 99.58 & 99.12 & 83.03 & 62.50 \\
\hline 38.00 & 64.72 & 93.80 & 97.49 & 97.69 & 97.74 & 97.74 & 97.62 & 97.17 & 81.90 & 62.31 \\
\hline 39.00 & 64.74 & 93.92 & 97.61 & 97.81 & 97.87 & 97.87 & 97.75 & 97.29 & 82.01 & 62.32 \\
\hline 40.00 & 64.33 & 91.15 & 94.51 & 94.71 & 94.77 & 94.76 & 94.64 & 94.20 & 80.21 & 62.09 \\
\hline 41.00 & 64.31 & 91.24 & 94.61 & 94.81 & 94.87 & 94.86 & 94.74 & 94.31 & 80.30 & 62.08 \\
\hline 42.00 & 63.68 & 87.31 & 90.22 & 90.39 & 90.45 & 90.44 & 90.33 & 89.95 & 77.69 & 61.67 \\
\hline 43.00 & 63.62 & 87.37 & 90.28 & 90.45 & 90.51 & 90.50 & 90.39 & 90.01 & 77.75 & 61.63 \\
\hline 44.00 & 60.20 & 61.78 & 62.27 & 62.44 & 62.51 & 62.50 & 62.36 & 62.08 & 61.35 & 59.56 \\
\hline 45.00 & 59.61 & 61.22 & 61.53 & 61.69 & 61.76 & 61.75 & 61.61 & 61.40 & 60.91 & 59.10 \\
\hline
\end{tabular}


Table B - 29. Temperature $\left({ }^{\circ} \mathrm{C}\right)$ map of the east side of plate $2 \mathrm{TT}$ at EOC $142 \mathrm{~A}$ (80.4 EFPD).

\begin{tabular}{|c||c|c|c|c|c|c|c|c|c|c|}
\hline \multirow{2}{*}{$\begin{array}{c}\text { Length of } \\
\text { Plate (in) }\end{array}$} & \multicolumn{10}{|c|}{ Width of Plate (inches) } \\
\hline 0.125 & 51.93 & 51.78 & 51.77 & 51.77 & 51.77 & 51.77 & 51.77 & 51.77 & 51.80 & 51.94 \\
\hline 1.00 & 52.94 & 52.48 & 52.66 & 52.68 & 52.69 & 52.69 & 52.68 & 52.60 & 52.41 & 52.83 \\
\hline 2.00 & 56.72 & 81.50 & 84.51 & 84.58 & 84.58 & 84.58 & 84.57 & 84.31 & 71.06 & 55.28 \\
\hline 3.00 & 57.06 & 81.75 & 84.79 & 84.87 & 84.87 & 84.87 & 84.86 & 84.58 & 71.34 & 55.60 \\
\hline 4.00 & 57.98 & 86.98 & 90.43 & 90.51 & 90.52 & 90.52 & 90.51 & 90.20 & 74.93 & 56.26 \\
\hline 5.00 & 58.24 & 87.15 & 90.61 & 90.70 & 90.71 & 90.71 & 90.69 & 90.38 & 75.12 & 56.51 \\
\hline 6.00 & 59.06 & 91.25 & 95.03 & 95.13 & 95.13 & 95.13 & 95.11 & 94.78 & 77.99 & 57.09 \\
\hline 7.00 & 59.34 & 91.45 & 95.23 & 95.33 & 95.34 & 95.34 & 95.31 & 94.98 & 78.20 & 57.34 \\
\hline 8.00 & 59.94 & 94.02 & 97.99 & 98.09 & 98.11 & 98.11 & 98.08 & 97.73 & 80.04 & 57.80 \\
\hline 9.00 & 60.18 & 94.19 & 98.16 & 98.27 & 98.28 & 98.28 & 98.25 & 97.89 & 80.25 & 58.08 \\
\hline 10.00 & 60.69 & 96.15 & 100.33 & 100.45 & 100.47 & 100.47 & 100.43 & 100.03 & 81.67 & 58.48 \\
\hline 11.00 & 60.96 & 96.34 & 100.55 & 100.69 & 100.71 & 100.71 & 100.66 & 100.25 & 81.88 & 58.71 \\
\hline 12.00 & 61.30 & 97.21 & 101.55 & 101.69 & 101.72 & 101.72 & 101.67 & 101.24 & 82.55 & 59.00 \\
\hline 13.00 & 61.53 & 97.38 & 101.75 & 101.90 & 101.93 & 101.93 & 101.87 & 101.43 & 82.75 & 59.23 \\
\hline 14.00 & 61.81 & 97.96 & 102.42 & 102.57 & 102.60 & 102.60 & 102.54 & 102.09 & 83.22 & 59.49 \\
\hline 15.00 & 62.04 & 98.12 & 102.62 & 102.78 & 102.81 & 102.81 & 102.74 & 102.29 & 83.41 & 59.69 \\
\hline 16.00 & 62.32 & 98.63 & 103.16 & 103.32 & 103.36 & 103.35 & 103.28 & 102.82 & 83.81 & 59.92 \\
\hline 17.00 & 62.54 & 98.83 & 103.35 & 103.52 & 103.56 & 103.56 & 103.48 & 103.01 & 84.01 & 60.19 \\
\hline 18.00 & 62.84 & 99.74 & 104.34 & 104.51 & 104.55 & 104.55 & 104.47 & 103.99 & 84.67 & 60.47 \\
\hline 19.00 & 63.06 & 99.95 & 104.54 & 104.72 & 104.76 & 104.76 & 104.68 & 104.19 & 84.87 & 60.66 \\
\hline 20.00 & 63.17 & 99.33 & 103.85 & 104.04 & 104.08 & 104.08 & 103.99 & 103.50 & 84.53 & 60.77 \\
\hline 21.00 & 63.32 & 99.48 & 104.00 & 104.19 & 104.24 & 104.24 & 104.14 & 103.65 & 84.68 & 60.92 \\
\hline 22.00 & 58.77 & 62.08 & 63.38 & 63.53 & 63.59 & 63.58 & 63.47 & 63.08 & 60.23 & 58.13 \\
\hline 22.50 & 57.31 & 56.88 & 57.08 & 57.20 & 57.26 & 57.25 & 57.14 & 56.97 & 56.85 & 57.10 \\
\hline
\end{tabular}


Table B - 30. Temperature $\left({ }^{\circ} \mathrm{C}\right)$ map of the west side of plate $2 \mathrm{TT}$ at EOC 142A (80.4 EFPD).

\begin{tabular}{|c||c|c|c|c|c|c|c|c|c|c|}
\hline \multirow{2}{*}{$\begin{array}{c}\text { Length of } \\
\text { Plate (in) }\end{array}$} & \multicolumn{10}{|c|}{ Width of Plate (inches) } \\
\hline 0.125 & 51.97 & 51.78 & 51.77 & 51.77 & 51.77 & 51.77 & 51.77 & 51.77 & 51.80 & 51.97 \\
\hline 1.00 & 53.07 & 52.49 & 52.66 & 52.68 & 52.69 & 52.69 & 52.68 & 52.60 & 52.41 & 52.90 \\
\hline 2.00 & 57.05 & 81.49 & 84.51 & 84.58 & 84.58 & 84.58 & 84.57 & 84.31 & 71.05 & 55.37 \\
\hline 3.00 & 57.41 & 81.76 & 84.79 & 84.86 & 84.87 & 84.87 & 84.86 & 84.58 & 71.33 & 55.70 \\
\hline 4.00 & 58.38 & 86.98 & 90.43 & 90.51 & 90.52 & 90.52 & 90.50 & 90.20 & 74.92 & 56.38 \\
\hline 5.00 & 58.66 & 87.16 & 90.61 & 90.70 & 90.70 & 90.70 & 90.69 & 90.38 & 75.11 & 56.63 \\
\hline 6.00 & 59.51 & 91.26 & 95.03 & 95.12 & 95.13 & 95.13 & 95.11 & 94.78 & 77.98 & 57.21 \\
\hline 7.00 & 59.80 & 91.45 & 95.22 & 95.32 & 95.33 & 95.33 & 95.31 & 94.97 & 78.20 & 57.46 \\
\hline 8.00 & 60.44 & 94.03 & 97.98 & 98.08 & 98.09 & 98.09 & 98.07 & 97.73 & 80.04 & 57.92 \\
\hline 9.00 & 60.69 & 94.20 & 98.15 & 98.25 & 98.27 & 98.27 & 98.24 & 97.89 & 80.24 & 58.27 \\
\hline 10.00 & 61.23 & 96.15 & 100.32 & 100.44 & 100.45 & 100.45 & 100.42 & 100.02 & 81.67 & 58.66 \\
\hline 11.00 & 61.51 & 96.35 & 100.54 & 100.67 & 100.69 & 100.68 & 100.65 & 100.25 & 81.88 & 58.87 \\
\hline 12.00 & 61.86 & 97.22 & 101.54 & 101.67 & 101.69 & 101.69 & 101.65 & 101.24 & 82.55 & 59.16 \\
\hline 13.00 & 62.10 & 97.39 & 101.74 & 101.88 & 101.90 & 101.90 & 101.85 & 101.43 & 82.75 & 59.40 \\
\hline 14.00 & 62.40 & 97.97 & 102.41 & 102.55 & 102.57 & 102.57 & 102.52 & 102.09 & 83.22 & 59.67 \\
\hline 15.00 & 62.63 & 98.14 & 102.61 & 102.75 & 102.78 & 102.77 & 102.72 & 102.28 & 83.41 & 59.85 \\
\hline 16.00 & 62.92 & 98.65 & 103.14 & 103.29 & 103.32 & 103.32 & 103.26 & 102.82 & 83.81 & 60.07 \\
\hline 17.00 & 63.14 & 98.85 & 103.34 & 103.49 & 103.52 & 103.52 & 103.46 & 103.01 & 84.02 & 60.39 \\
\hline 18.00 & 63.45 & 99.76 & 104.32 & 104.48 & 104.51 & 104.50 & 104.44 & 103.99 & 84.68 & 60.69 \\
\hline 19.00 & 63.68 & 99.97 & 104.53 & 104.69 & 104.72 & 104.71 & 104.65 & 104.19 & 84.88 & 60.88 \\
\hline 20.00 & 63.78 & 99.35 & 103.83 & 104.00 & 104.03 & 104.03 & 103.96 & 103.50 & 84.54 & 60.95 \\
\hline 21.00 & 63.94 & 99.51 & 103.98 & 104.15 & 104.19 & 104.18 & 104.11 & 103.65 & 84.69 & 61.09 \\
\hline 22.00 & 59.14 & 62.10 & 63.36 & 63.50 & 63.54 & 63.54 & 63.45 & 63.07 & 60.26 & 58.27 \\
\hline 22.50 & 57.66 & 56.90 & 57.07 & 57.17 & 57.21 & 57.20 & 57.12 & 56.97 & 56.88 & 57.30 \\
\hline
\end{tabular}


Table B - 31. Temperature $\left({ }^{\circ} \mathrm{C}\right)$ map of the east side of plate $2 \mathrm{BZ}$ at EOC $142 \mathrm{~A}$ (80.4 EFPD).

\begin{tabular}{|c||c|c|c|c|c|c|c|c|c|c|}
\hline \multirow{2}{*}{$\begin{array}{c}\text { Length of } \\
\text { Plate (in) }\end{array}$} & \multicolumn{9}{|c|}{ Width of Plate (inches) } \\
\hline 22.625 & 57.41 & 56.92 & 57.12 & 57.24 & 57.30 & 57.29 & 57.18 & 57.01 & 56.88 & 57.20 \\
\hline 23.00 & 58.54 & 61.00 & 62.05 & 62.20 & 62.26 & 62.25 & 62.14 & 61.80 & 59.54 & 57.99 \\
\hline 24.00 & 62.37 & 91.28 & 94.81 & 94.97 & 95.01 & 95.01 & 94.92 & 94.53 & 79.25 & 60.42 \\
\hline 25.00 & 62.52 & 91.43 & 94.95 & 95.11 & 95.16 & 95.16 & 95.06 & 94.67 & 79.42 & 60.57 \\
\hline 26.00 & 62.59 & 91.11 & 94.59 & 94.76 & 94.81 & 94.81 & 94.71 & 94.31 & 79.27 & 60.69 \\
\hline 27.00 & 62.71 & 91.24 & 94.73 & 94.90 & 94.95 & 94.95 & 94.84 & 94.45 & 79.42 & 60.82 \\
\hline 28.00 & 62.78 & 91.03 & 94.49 & 94.66 & 94.71 & 94.71 & 94.61 & 94.21 & 79.32 & 60.89 \\
\hline 29.00 & 62.91 & 91.18 & 94.64 & 94.81 & 94.87 & 94.86 & 94.76 & 94.36 & 79.47 & 60.98 \\
\hline 30.00 & 63.03 & 91.42 & 94.88 & 95.08 & 95.14 & 95.14 & 95.01 & 94.61 & 79.68 & 61.09 \\
\hline 31.00 & 63.12 & 91.55 & 95.03 & 95.23 & 95.29 & 95.29 & 95.16 & 94.73 & 79.82 & 61.19 \\
\hline 32.00 & 63.14 & 91.20 & 94.63 & 94.83 & 94.89 & 94.89 & 94.76 & 94.34 & 79.63 & 61.23 \\
\hline 33.00 & 63.23 & 91.34 & 94.78 & 94.99 & 95.06 & 95.05 & 94.92 & 94.48 & 79.76 & 61.30 \\
\hline 34.00 & 63.22 & 90.91 & 94.29 & 94.50 & 94.57 & 94.56 & 94.43 & 94.01 & 79.52 & 61.32 \\
\hline 35.00 & 63.28 & 91.03 & 94.43 & 94.64 & 94.71 & 94.71 & 94.57 & 94.13 & 79.64 & 61.40 \\
\hline 36.00 & 63.28 & 90.69 & 94.05 & 94.26 & 94.33 & 94.32 & 94.18 & 93.76 & 79.46 & 61.42 \\
\hline 37.00 & 63.33 & 90.82 & 94.20 & 94.41 & 94.48 & 94.47 & 94.33 & 93.89 & 79.58 & 61.46 \\
\hline 38.00 & 63.14 & 89.39 & 92.61 & 92.81 & 92.87 & 92.87 & 92.74 & 92.33 & 78.65 & 61.33 \\
\hline 39.00 & 63.17 & 89.50 & 92.72 & 92.91 & 92.97 & 92.97 & 92.84 & 92.44 & 78.75 & 61.35 \\
\hline 40.00 & 62.93 & 87.76 & 90.83 & 91.03 & 91.09 & 91.09 & 90.96 & 90.56 & 77.61 & 61.21 \\
\hline 41.00 & 62.93 & 87.85 & 90.92 & 91.12 & 91.18 & 91.18 & 91.05 & 90.65 & 77.70 & 61.20 \\
\hline 42.00 & 62.49 & 84.92 & 87.74 & 87.93 & 88.00 & 88.00 & 87.86 & 87.47 & 75.76 & 60.91 \\
\hline 43.00 & 62.46 & 84.99 & 87.80 & 88.00 & 88.06 & 88.06 & 87.93 & 87.54 & 75.82 & 60.87 \\
\hline 44.00 & 59.38 & 60.59 & 61.05 & 61.23 & 61.31 & 61.31 & 61.15 & 60.87 & 60.22 & 58.93 \\
\hline 45.00 & 58.84 & 60.05 & 60.34 & 60.51 & 60.59 & 60.58 & 60.43 & 60.21 & 59.79 & 58.49 \\
\hline
\end{tabular}


Table B - 32. Temperature $\left({ }^{\circ} \mathrm{C}\right)$ map of the west side of plate $2 \mathrm{BZ}$ at EOC $142 \mathrm{~A}$ (80.4 EFPD).

\begin{tabular}{|c||c|c|c|c|c|c|c|c|c|c|}
\hline \multirow{2}{*}{$\begin{array}{c}\text { Length of } \\
\text { Plate (in) }\end{array}$} & \multicolumn{10}{|c|}{ Width of Plate (inches) } \\
\hline 22.625 & 57.73 & 56.94 & 57.11 & 57.21 & 57.25 & 57.25 & 57.16 & 57.00 & 56.91 & 57.36 \\
\hline 23.00 & 58.90 & 61.02 & 62.04 & 62.17 & 62.21 & 62.20 & 62.11 & 61.79 & 59.56 & 58.12 \\
\hline 24.00 & 62.92 & 91.30 & 94.78 & 94.92 & 94.96 & 94.95 & 94.88 & 94.52 & 79.27 & 60.57 \\
\hline 25.00 & 63.06 & 91.45 & 94.93 & 95.07 & 95.10 & 95.10 & 95.03 & 94.66 & 79.44 & 60.73 \\
\hline 26.00 & 63.13 & 91.12 & 94.57 & 94.71 & 94.75 & 94.74 & 94.67 & 94.30 & 79.28 & 60.89 \\
\hline 27.00 & 63.24 & 91.26 & 94.70 & 94.85 & 94.88 & 94.88 & 94.80 & 94.44 & 79.43 & 61.02 \\
\hline 28.00 & 63.30 & 91.05 & 94.46 & 94.61 & 94.64 & 94.64 & 94.56 & 94.20 & 79.33 & 61.07 \\
\hline 29.00 & 63.42 & 91.20 & 94.61 & 94.76 & 94.79 & 94.79 & 94.71 & 94.35 & 79.48 & 61.13 \\
\hline 30.00 & 63.53 & 91.44 & 94.86 & 95.01 & 95.06 & 95.05 & 94.96 & 94.59 & 79.69 & 61.23 \\
\hline 31.00 & 63.62 & 91.57 & 94.99 & 95.16 & 95.20 & 95.20 & 95.11 & 94.72 & 79.83 & 61.33 \\
\hline 32.00 & 63.62 & 91.21 & 94.59 & 94.76 & 94.80 & 94.80 & 94.70 & 94.33 & 79.64 & 61.36 \\
\hline 33.00 & 63.70 & 91.35 & 94.75 & 94.91 & 94.96 & 94.96 & 94.86 & 94.47 & 79.78 & 61.41 \\
\hline 34.00 & 63.67 & 90.92 & 94.26 & 94.42 & 94.47 & 94.47 & 94.37 & 93.99 & 79.53 & 61.43 \\
\hline 35.00 & 63.72 & 91.04 & 94.39 & 94.56 & 94.61 & 94.60 & 94.51 & 94.11 & 79.65 & 61.54 \\
\hline 36.00 & 63.70 & 90.71 & 94.01 & 94.18 & 94.23 & 94.22 & 94.12 & 93.74 & 79.47 & 61.56 \\
\hline 37.00 & 63.73 & 90.83 & 94.15 & 94.33 & 94.37 & 94.37 & 94.27 & 93.87 & 79.59 & 61.58 \\
\hline 38.00 & 63.52 & 89.40 & 92.57 & 92.73 & 92.77 & 92.77 & 92.68 & 92.31 & 78.65 & 61.41 \\
\hline 39.00 & 63.53 & 89.50 & 92.68 & 92.83 & 92.88 & 92.87 & 92.78 & 92.41 & 78.75 & 61.41 \\
\hline 40.00 & 63.26 & 87.77 & 90.79 & 90.95 & 90.99 & 90.99 & 90.89 & 90.53 & 77.62 & 61.27 \\
\hline 41.00 & 63.24 & 87.86 & 90.88 & 91.03 & 91.08 & 91.08 & 90.98 & 90.62 & 77.70 & 61.26 \\
\hline 42.00 & 62.76 & 84.93 & 87.69 & 87.85 & 87.90 & 87.89 & 87.79 & 87.44 & 75.77 & 60.95 \\
\hline 43.00 & 62.70 & 84.99 & 87.76 & 87.91 & 87.96 & 87.95 & 87.86 & 87.51 & 75.83 & 60.90 \\
\hline 44.00 & 59.45 & 60.59 & 61.01 & 61.16 & 61.22 & 61.21 & 61.09 & 60.84 & 60.23 & 58.93 \\
\hline 45.00 & 58.89 & 60.05 & 60.30 & 60.44 & 60.50 & 60.49 & 60.37 & 60.19 & 59.81 & 58.49 \\
\hline
\end{tabular}


Table B - 33. Temperature $\left({ }^{\circ} \mathrm{C}\right)$ map of the east side of plate 2TT at BOC $142 \mathrm{~B}$ (80.4 EFPD).

\begin{tabular}{|c||c|c|c|c|c|c|c|c|c|c|}
\hline \multirow{2}{*}{$\begin{array}{c}\text { Length of } \\
\text { Plate (in) }\end{array}$} & \multicolumn{10}{|c|}{ Width of Plate (inches) } \\
\hline 0.125 & 51.94 & 51.78 & 51.77 & 51.77 & 51.77 & 51.77 & 51.77 & 51.77 & 51.81 & 51.95 \\
\hline 1.00 & 52.89 & 52.33 & 52.45 & 52.47 & 52.47 & 52.47 & 52.46 & 52.41 & 52.32 & 52.82 \\
\hline 2.00 & 56.00 & 74.45 & 76.81 & 76.87 & 76.87 & 76.87 & 76.87 & 76.64 & 66.46 & 54.97 \\
\hline 3.00 & 56.35 & 74.68 & 77.06 & 77.13 & 77.13 & 77.13 & 77.12 & 76.88 & 66.71 & 55.30 \\
\hline 4.00 & 57.22 & 79.24 & 82.00 & 82.07 & 82.08 & 82.08 & 82.06 & 81.81 & 69.80 & 55.97 \\
\hline 5.00 & 57.49 & 79.40 & 82.17 & 82.24 & 82.25 & 82.25 & 82.23 & 81.97 & 69.99 & 56.23 \\
\hline 6.00 & 58.27 & 82.85 & 85.90 & 85.98 & 85.99 & 85.98 & 85.97 & 85.68 & 72.39 & 56.84 \\
\hline 7.00 & 58.57 & 83.03 & 86.08 & 86.17 & 86.18 & 86.18 & 86.15 & 85.86 & 72.59 & 57.11 \\
\hline 8.00 & 59.18 & 85.34 & 88.56 & 88.65 & 88.66 & 88.66 & 88.63 & 88.33 & 74.23 & 57.60 \\
\hline 9.00 & 59.43 & 85.50 & 88.72 & 88.82 & 88.83 & 88.83 & 88.80 & 88.49 & 74.44 & 57.94 \\
\hline 10.00 & 59.98 & 87.28 & 90.63 & 90.73 & 90.75 & 90.75 & 90.72 & 90.40 & 75.74 & 58.38 \\
\hline 11.00 & 60.27 & 87.48 & 90.82 & 90.93 & 90.95 & 90.94 & 90.91 & 90.58 & 75.95 & 58.63 \\
\hline 12.00 & 60.82 & 89.32 & 92.80 & 92.91 & 92.93 & 92.93 & 92.89 & 92.55 & 77.30 & 59.09 \\
\hline 13.00 & 61.07 & 89.50 & 92.97 & 93.09 & 93.11 & 93.11 & 93.06 & 92.72 & 77.50 & 59.36 \\
\hline 14.00 & 61.52 & 90.81 & 94.38 & 94.50 & 94.52 & 94.52 & 94.47 & 94.12 & 78.49 & 59.75 \\
\hline 15.00 & 61.78 & 91.00 & 94.56 & 94.68 & 94.71 & 94.70 & 94.65 & 94.30 & 78.69 & 59.97 \\
\hline 16.00 & 62.13 & 91.59 & 95.18 & 95.30 & 95.33 & 95.33 & 95.27 & 94.92 & 79.19 & 60.27 \\
\hline 17.00 & 62.37 & 91.76 & 95.35 & 95.49 & 95.52 & 95.52 & 95.45 & 95.09 & 79.41 & 60.59 \\
\hline 18.00 & 62.69 & 92.48 & 96.12 & 96.26 & 96.29 & 96.29 & 96.22 & 95.85 & 79.99 & 60.92 \\
\hline 19.00 & 62.93 & 92.67 & 96.31 & 96.45 & 96.49 & 96.49 & 96.42 & 96.04 & 80.19 & 61.12 \\
\hline 20.00 & 63.35 & 93.90 & 97.64 & 97.80 & 97.84 & 97.84 & 97.75 & 97.35 & 81.12 & 61.44 \\
\hline 21.00 & 63.57 & 94.08 & 97.84 & 98.00 & 98.05 & 98.04 & 97.96 & 97.53 & 81.31 & 61.65 \\
\hline 22.00 & 59.18 & 61.48 & 62.57 & 62.71 & 62.77 & 62.76 & 62.65 & 62.31 & 59.96 & 58.72 \\
\hline 22.50 & 57.71 & 56.99 & 57.14 & 57.26 & 57.31 & 57.31 & 57.20 & 57.05 & 56.99 & 57.60 \\
\hline
\end{tabular}


Table B - 34. Temperature $\left({ }^{\circ} \mathrm{C}\right)$ map of the west side of plate 2TT at BOC 142B (80.4 EFPD).

\begin{tabular}{|c||c|c|c|c|c|c|c|c|c|c|}
\hline \multirow{2}{*}{$\begin{array}{c}\text { Length of } \\
\text { Plate (in) }\end{array}$} & \multicolumn{10}{|c|}{ Width of Plate (inches) } \\
\hline 0.125 & 51.98 & 51.78 & 51.77 & 51.77 & 51.77 & 51.77 & 51.77 & 51.77 & 51.81 & 51.98 \\
\hline 1.00 & 53.11 & 52.34 & 52.45 & 52.47 & 52.47 & 52.47 & 52.47 & 52.41 & 52.32 & 52.94 \\
\hline 2.00 & 56.79 & 74.47 & 76.83 & 76.89 & 76.89 & 76.89 & 76.88 & 76.66 & 66.46 & 55.36 \\
\hline 3.00 & 57.20 & 74.73 & 77.10 & 77.17 & 77.17 & 77.17 & 77.16 & 76.93 & 66.73 & 55.71 \\
\hline 4.00 & 58.24 & 79.32 & 82.07 & 82.15 & 82.15 & 82.15 & 82.14 & 81.88 & 69.85 & 56.49 \\
\hline 5.00 & 58.55 & 79.52 & 82.28 & 82.36 & 82.37 & 82.37 & 82.35 & 82.08 & 70.08 & 56.76 \\
\hline 6.00 & 59.52 & 83.01 & 86.05 & 86.13 & 86.14 & 86.14 & 86.12 & 85.83 & 72.51 & 57.46 \\
\hline 7.00 & 59.87 & 83.25 & 86.29 & 86.38 & 86.39 & 86.39 & 86.36 & 86.07 & 72.76 & 57.74 \\
\hline 8.00 & 60.63 & 85.60 & 88.82 & 88.91 & 88.93 & 88.93 & 88.90 & 88.59 & 74.45 & 58.31 \\
\hline 9.00 & 60.93 & 85.82 & 89.04 & 89.14 & 89.16 & 89.16 & 89.12 & 88.81 & 74.71 & 58.75 \\
\hline 10.00 & 61.64 & 87.67 & 91.01 & 91.12 & 91.14 & 91.14 & 91.10 & 90.77 & 76.06 & 59.26 \\
\hline 11.00 & 61.98 & 87.93 & 91.28 & 91.39 & 91.41 & 91.41 & 91.36 & 91.03 & 76.34 & 59.49 \\
\hline 12.00 & 62.65 & 89.84 & 93.32 & 93.44 & 93.46 & 93.46 & 93.41 & 93.07 & 77.74 & 60.00 \\
\hline 13.00 & 62.95 & 90.09 & 93.57 & 93.69 & 93.72 & 93.72 & 93.66 & 93.31 & 78.01 & 60.31 \\
\hline 14.00 & 63.51 & 91.48 & 95.05 & 95.17 & 95.20 & 95.20 & 95.14 & 94.79 & 79.07 & 60.77 \\
\hline 15.00 & 63.82 & 91.74 & 95.30 & 95.44 & 95.47 & 95.47 & 95.40 & 95.04 & 79.34 & 60.99 \\
\hline 16.00 & 64.27 & 92.42 & 96.01 & 96.15 & 96.19 & 96.18 & 96.11 & 95.75 & 79.91 & 61.32 \\
\hline 17.00 & 64.55 & 92.68 & 96.28 & 96.44 & 96.48 & 96.48 & 96.39 & 96.00 & 80.21 & 61.74 \\
\hline 18.00 & 64.94 & 93.48 & 97.18 & 97.35 & 97.39 & 97.39 & 97.30 & 96.89 & 80.87 & 62.13 \\
\hline 19.00 & 65.23 & 93.75 & 97.49 & 97.66 & 97.71 & 97.70 & 97.61 & 97.19 & 81.15 & 62.34 \\
\hline 20.00 & 65.74 & 95.08 & 98.98 & 99.17 & 99.22 & 99.21 & 99.11 & 98.68 & 82.16 & 62.64 \\
\hline 21.00 & 66.01 & 95.36 & 99.28 & 99.47 & 99.52 & 99.52 & 99.41 & 98.97 & 82.43 & 62.87 \\
\hline 22.00 & 60.62 & 62.65 & 63.75 & 63.92 & 63.98 & 63.97 & 63.85 & 63.48 & 61.10 & 59.43 \\
\hline 22.50 & 58.79 & 58.21 & 58.38 & 58.52 & 58.58 & 58.58 & 58.45 & 58.27 & 58.17 & 58.18 \\
\hline
\end{tabular}


Table B - 35. Temperature $\left({ }^{\circ} \mathrm{C}\right)$ map of the east side of plate $2 \mathrm{BZ}$ at BOC $142 \mathrm{~B}$ (80.4 EFPD).

\begin{tabular}{|c||c|c|c|c|c|c|c|c|c|c|}
\hline \multirow{2}{*}{$\begin{array}{c}\text { Length of } \\
\text { Plate (in) }\end{array}$} & \multicolumn{10}{|c|}{ Width of Plate (inches) } \\
\hline 22.625 & 57.83 & 57.02 & 57.18 & 57.30 & 57.35 & 57.35 & 57.24 & 57.08 & 57.02 & 57.72 \\
\hline 23.00 & 58.99 & 60.47 & 61.33 & 61.47 & 61.52 & 61.52 & 61.41 & 61.11 & 59.33 & 58.62 \\
\hline 24.00 & 62.75 & 86.12 & 89.12 & 89.27 & 89.31 & 89.31 & 89.22 & 88.88 & 76.07 & 61.28 \\
\hline 25.00 & 62.95 & 86.31 & 89.31 & 89.46 & 89.51 & 89.50 & 89.41 & 89.06 & 76.27 & 61.47 \\
\hline 26.00 & 63.07 & 86.33 & 89.32 & 89.47 & 89.52 & 89.51 & 89.42 & 89.07 & 76.36 & 61.65 \\
\hline 27.00 & 63.22 & 86.49 & 89.47 & 89.63 & 89.68 & 89.67 & 89.58 & 89.23 & 76.54 & 61.83 \\
\hline 28.00 & 63.34 & 86.42 & 89.38 & 89.54 & 89.59 & 89.59 & 89.49 & 89.14 & 76.55 & 61.92 \\
\hline 29.00 & 63.49 & 86.58 & 89.55 & 89.71 & 89.76 & 89.76 & 89.66 & 89.30 & 76.72 & 62.02 \\
\hline 30.00 & 63.58 & 86.48 & 89.42 & 89.59 & 89.64 & 89.64 & 89.53 & 89.18 & 76.71 & 62.12 \\
\hline 31.00 & 63.70 & 86.63 & 89.57 & 89.74 & 89.80 & 89.79 & 89.68 & 89.32 & 76.87 & 62.27 \\
\hline 32.00 & 63.70 & 86.10 & 88.98 & 89.16 & 89.21 & 89.21 & 89.10 & 88.74 & 76.57 & 62.29 \\
\hline 33.00 & 63.81 & 86.25 & 89.15 & 89.32 & 89.38 & 89.37 & 89.26 & 88.90 & 76.72 & 62.37 \\
\hline 34.00 & 63.78 & 85.74 & 88.58 & 88.75 & 88.81 & 88.81 & 88.69 & 88.33 & 76.42 & 62.36 \\
\hline 35.00 & 63.87 & 85.87 & 88.72 & 88.89 & 88.95 & 88.95 & 88.83 & 88.47 & 76.57 & 62.50 \\
\hline 36.00 & 63.77 & 85.14 & 87.91 & 88.09 & 88.15 & 88.14 & 88.02 & 87.66 & 76.12 & 62.47 \\
\hline 37.00 & 63.84 & 85.28 & 88.05 & 88.23 & 88.29 & 88.29 & 88.17 & 87.80 & 76.26 & 62.50 \\
\hline 38.00 & 63.62 & 83.85 & 86.48 & 86.66 & 86.73 & 86.72 & 86.60 & 86.24 & 75.32 & 62.30 \\
\hline 39.00 & 63.66 & 83.96 & 86.60 & 86.78 & 86.84 & 86.84 & 86.71 & 86.36 & 75.43 & 62.33 \\
\hline 40.00 & 63.39 & 82.24 & 84.72 & 84.90 & 84.96 & 84.95 & 84.83 & 84.48 & 74.33 & 62.17 \\
\hline 41.00 & 63.39 & 82.33 & 84.81 & 84.99 & 85.06 & 85.05 & 84.93 & 84.58 & 74.42 & 62.17 \\
\hline 42.00 & 62.97 & 79.79 & 82.03 & 82.21 & 82.28 & 82.27 & 82.14 & 81.81 & 72.75 & 61.84 \\
\hline 43.00 & 62.93 & 79.86 & 82.10 & 82.28 & 82.35 & 82.34 & 82.21 & 81.88 & 72.81 & 61.80 \\
\hline 44.00 & 60.29 & 61.17 & 61.56 & 61.74 & 61.82 & 61.81 & 61.65 & 61.41 & 60.83 & 60.02 \\
\hline 45.00 & 59.78 & 60.76 & 61.03 & 61.20 & 61.28 & 61.27 & 61.12 & 60.92 & 60.49 & 59.61 \\
\hline
\end{tabular}


Table B - 36. Temperature $\left({ }^{\circ} \mathrm{C}\right)$ map of the west side of plate $2 \mathrm{BZ}$ at BOC $142 \mathrm{~B}$ (80.4 EFPD).

\begin{tabular}{|c||c|c|c|c|c|c|c|c|c|c|}
\hline \multirow{2}{*}{$\begin{array}{c}\text { Length of } \\
\text { Plate (in) }\end{array}$} & \multicolumn{10}{|c|}{ Width of Plate (inches) } \\
\hline 22.625 & 58.91 & 58.25 & 58.43 & 58.57 & 58.63 & 58.62 & 58.50 & 58.32 & 58.21 & 58.27 \\
\hline 23.00 & 60.45 & 61.70 & 62.57 & 62.73 & 62.79 & 62.79 & 62.66 & 62.34 & 60.52 & 59.34 \\
\hline 24.00 & 65.25 & 87.65 & 90.66 & 90.83 & 90.88 & 90.88 & 90.77 & 90.40 & 77.39 & 62.54 \\
\hline 25.00 & 65.47 & 87.92 & 90.94 & 91.11 & 91.16 & 91.16 & 91.05 & 90.67 & 77.68 & 62.75 \\
\hline 26.00 & 65.59 & 88.03 & 91.03 & 91.21 & 91.27 & 91.26 & 91.15 & 90.77 & 77.84 & 62.99 \\
\hline 27.00 & 65.77 & 88.28 & 91.28 & 91.46 & 91.52 & 91.52 & 91.39 & 91.01 & 78.10 & 63.22 \\
\hline 28.00 & 65.91 & 88.30 & 91.29 & 91.47 & 91.53 & 91.53 & 91.40 & 91.02 & 78.19 & 63.28 \\
\hline 29.00 & 66.10 & 88.57 & 91.56 & 91.75 & 91.82 & 91.81 & 91.68 & 91.29 & 78.46 & 63.35 \\
\hline 30.00 & 66.21 & 88.56 & 91.53 & 91.74 & 91.81 & 91.80 & 91.66 & 91.26 & 78.53 & 63.44 \\
\hline 31.00 & 66.35 & 88.80 & 91.79 & 92.00 & 92.08 & 92.07 & 91.92 & 91.50 & 78.77 & 63.62 \\
\hline 32.00 & 66.33 & 88.37 & 91.30 & 91.52 & 91.59 & 91.58 & 91.43 & 91.01 & 78.55 & 63.62 \\
\hline 33.00 & 66.47 & 88.63 & 91.59 & 91.80 & 91.88 & 91.87 & 91.72 & 91.29 & 78.80 & 63.69 \\
\hline 34.00 & 66.39 & 88.21 & 91.11 & 91.33 & 91.41 & 91.40 & 91.24 & 90.82 & 78.58 & 63.67 \\
\hline 35.00 & 66.50 & 88.42 & 91.35 & 91.57 & 91.65 & 91.65 & 91.48 & 91.06 & 78.80 & 63.86 \\
\hline 36.00 & 66.33 & 87.78 & 90.62 & 90.85 & 90.93 & 90.92 & 90.75 & 90.33 & 78.43 & 63.82 \\
\hline 37.00 & 66.41 & 88.01 & 90.87 & 91.10 & 91.18 & 91.17 & 91.00 & 90.58 & 78.64 & 63.82 \\
\hline 38.00 & 66.11 & 86.67 & 89.37 & 89.59 & 89.68 & 89.67 & 89.50 & 89.08 & 77.79 & 63.53 \\
\hline 39.00 & 66.16 & 86.85 & 89.56 & 89.79 & 89.88 & 89.87 & 89.70 & 89.27 & 77.96 & 63.55 \\
\hline 40.00 & 65.78 & 85.23 & 87.75 & 87.97 & 88.05 & 88.04 & 87.88 & 87.48 & 76.93 & 63.34 \\
\hline 41.00 & 65.78 & 85.38 & 87.91 & 88.14 & 88.22 & 88.22 & 88.04 & 87.64 & 77.07 & 63.35 \\
\hline 42.00 & 65.23 & 82.94 & 85.24 & 85.46 & 85.54 & 85.54 & 85.37 & 84.98 & 75.48 & 62.92 \\
\hline 43.00 & 65.18 & 83.06 & 85.36 & 85.58 & 85.66 & 85.66 & 85.49 & 85.10 & 75.59 & 62.88 \\
\hline 44.00 & 61.97 & 63.82 & 64.26 & 64.47 & 64.57 & 64.56 & 64.37 & 64.07 & 63.40 & 60.85 \\
\hline 45.00 & 61.35 & 63.43 & 63.75 & 63.95 & 64.04 & 64.03 & 63.84 & 63.60 & 63.08 & 60.40 \\
\hline
\end{tabular}


Table B - 37. Temperature $\left({ }^{\circ} \mathrm{C}\right)$ map of the east side of plate $2 \mathrm{TT}$ at MOC $142 \mathrm{~B}$ (96.4 EFPD).

\begin{tabular}{|c||c|c|c|c|c|c|c|c|c|c|}
\hline \multirow{2}{*}{$\begin{array}{c}\text { Length of } \\
\text { Plate (in) }\end{array}$} & \multicolumn{10}{|c|}{ Width of Plate (inches) } \\
\hline 0.125 & 51.94 & 51.78 & 51.77 & 51.77 & 51.77 & 51.77 & 51.77 & 51.77 & 51.81 & 51.95 \\
\hline 1.00 & 52.89 & 52.34 & 52.46 & 52.48 & 52.48 & 52.48 & 52.47 & 52.42 & 52.32 & 52.82 \\
\hline 2.00 & 56.06 & 74.86 & 77.26 & 77.32 & 77.32 & 77.32 & 77.31 & 77.09 & 66.73 & 55.01 \\
\hline 3.00 & 56.40 & 75.09 & 77.50 & 77.56 & 77.57 & 77.57 & 77.56 & 77.32 & 66.97 & 55.33 \\
\hline 4.00 & 57.19 & 79.01 & 81.76 & 81.83 & 81.83 & 81.83 & 81.82 & 81.56 & 69.66 & 55.95 \\
\hline 5.00 & 57.46 & 79.18 & 81.92 & 82.00 & 82.01 & 82.01 & 81.99 & 81.72 & 69.84 & 56.21 \\
\hline 6.00 & 58.27 & 82.88 & 85.93 & 86.01 & 86.02 & 86.02 & 86.00 & 85.71 & 72.41 & 56.83 \\
\hline 7.00 & 58.56 & 83.06 & 86.12 & 86.20 & 86.21 & 86.21 & 86.19 & 85.90 & 72.61 & 57.10 \\
\hline 8.00 & 59.13 & 85.07 & 88.27 & 88.36 & 88.38 & 88.37 & 88.35 & 88.04 & 74.05 & 57.57 \\
\hline 9.00 & 59.38 & 85.23 & 88.43 & 88.53 & 88.54 & 88.54 & 88.51 & 88.20 & 74.26 & 57.90 \\
\hline 10.00 & 59.84 & 86.56 & 89.85 & 89.95 & 89.97 & 89.97 & 89.93 & 89.62 & 75.24 & 58.28 \\
\hline 11.00 & 60.13 & 86.75 & 90.04 & 90.14 & 90.16 & 90.16 & 90.12 & 89.80 & 75.45 & 58.52 \\
\hline 12.00 & 60.56 & 87.97 & 91.34 & 91.45 & 91.47 & 91.47 & 91.43 & 91.10 & 76.36 & 58.90 \\
\hline 13.00 & 60.81 & 88.14 & 91.51 & 91.62 & 91.64 & 91.64 & 91.59 & 91.26 & 76.56 & 59.16 \\
\hline 14.00 & 61.14 & 88.77 & 92.17 & 92.29 & 92.31 & 92.31 & 92.26 & 91.93 & 77.08 & 59.48 \\
\hline 15.00 & 61.39 & 88.94 & 92.34 & 92.46 & 92.49 & 92.48 & 92.43 & 92.09 & 77.27 & 59.69 \\
\hline 16.00 & 61.75 & 89.69 & 93.14 & 93.26 & 93.29 & 93.29 & 93.23 & 92.88 & 77.86 & 59.99 \\
\hline 17.00 & 61.98 & 89.87 & 93.31 & 93.43 & 93.46 & 93.46 & 93.40 & 93.05 & 78.08 & 60.30 \\
\hline 18.00 & 62.14 & 89.50 & 92.90 & 93.03 & 93.06 & 93.06 & 93.00 & 92.64 & 77.92 & 60.51 \\
\hline 19.00 & 62.34 & 89.67 & 93.06 & 93.19 & 93.23 & 93.22 & 93.16 & 92.80 & 78.10 & 60.69 \\
\hline 20.00 & 62.70 & 90.55 & 93.99 & 94.13 & 94.17 & 94.17 & 94.10 & 93.73 & 78.78 & 60.96 \\
\hline 21.00 & 62.90 & 90.71 & 94.15 & 94.30 & 94.34 & 94.34 & 94.26 & 93.89 & 78.96 & 61.15 \\
\hline 22.00 & 58.91 & 60.86 & 61.85 & 61.99 & 62.04 & 62.04 & 61.93 & 61.61 & 59.49 & 58.49 \\
\hline 22.50 & 57.57 & 56.78 & 56.92 & 57.03 & 57.08 & 57.07 & 56.97 & 56.83 & 56.80 & 57.48 \\
\hline
\end{tabular}


Table B - 38. Temperature $\left({ }^{\circ} \mathrm{C}\right)$ map of the west side of plate 2TT at MOC 142B (96.4 EFPD).

\begin{tabular}{|c||c|c|c|c|c|c|c|c|c|c|}
\hline \multirow{2}{*}{$\begin{array}{c}\text { Length of } \\
\text { Plate (in) }\end{array}$} & \multicolumn{10}{|c|}{ Width of Plate (inches) } \\
\hline & 0.05 & 0.25 & 0.50 & 0.75 & 1.00 & 1.25 & 1.50 & 1.75 & 2.00 & 2.24 \\
\hline 0.125 & 51.98 & 51.78 & 51.77 & 51.77 & 51.77 & 51.77 & 51.77 & 51.77 & 51.81 & 51.98 \\
\hline 1.00 & 53.12 & 52.35 & 52.47 & 52.48 & 52.48 & 52.48 & 52.48 & 52.42 & 52.33 & 52.95 \\
\hline 2.00 & 56.86 & 74.88 & 77.27 & 77.33 & 77.34 & 77.34 & 77.33 & 77.10 & 66.73 & 55.40 \\
\hline 3.00 & 57.25 & 75.13 & 77.54 & 77.61 & 77.61 & 77.61 & 77.60 & 77.36 & 67.00 & 55.75 \\
\hline 4.00 & 58.20 & 79.09 & 81.83 & 81.90 & 81.91 & 81.91 & 81.89 & 81.64 & 69.71 & 56.47 \\
\hline 5.00 & 58.52 & 79.29 & 82.04 & 82.11 & 82.12 & 82.12 & 82.10 & 81.84 & 69.93 & 56.75 \\
\hline 6.00 & 59.52 & 83.04 & 86.08 & 86.17 & 86.18 & 86.18 & 86.16 & 85.87 & 72.53 & 57.45 \\
\hline 7.00 & 59.86 & 83.28 & 86.32 & 86.41 & 86.43 & 86.42 & 86.40 & 86.10 & 72.78 & 57.73 \\
\hline 8.00 & 60.58 & 85.34 & 88.53 & 88.63 & 88.64 & 88.64 & 88.61 & 88.30 & 74.27 & 58.27 \\
\hline 9.00 & 60.87 & 85.56 & 88.75 & 88.85 & 88.87 & 88.87 & 88.83 & 88.52 & 74.52 & 58.71 \\
\hline 10.00 & 61.46 & 86.94 & 90.23 & 90.34 & 90.36 & 90.35 & 90.31 & 89.99 & 75.56 & 59.13 \\
\hline 11.00 & 61.79 & 87.20 & 90.49 & 90.60 & 90.62 & 90.62 & 90.57 & 90.25 & 75.83 & 59.36 \\
\hline 12.00 & 62.33 & 88.49 & 91.86 & 91.97 & 92.00 & 91.99 & 91.95 & 91.61 & 76.80 & 59.78 \\
\hline 13.00 & 62.62 & 88.73 & 92.09 & 92.21 & 92.24 & 92.24 & 92.18 & 91.84 & 77.06 & 60.08 \\
\hline 14.00 & 63.06 & 89.43 & 92.83 & 92.96 & 92.99 & 92.99 & 92.93 & 92.58 & 77.64 & 60.46 \\
\hline 15.00 & 63.34 & 89.68 & 93.08 & 93.21 & 93.24 & 93.24 & 93.17 & 92.82 & 77.90 & 60.67 \\
\hline 16.00 & 63.79 & 90.51 & 93.96 & 94.09 & 94.13 & 94.12 & 94.06 & 93.70 & 78.57 & 60.99 \\
\hline 17.00 & 64.07 & 90.76 & 94.21 & 94.35 & 94.38 & 94.38 & 94.31 & 93.94 & 78.86 & 61.40 \\
\hline 18.00 & 64.27 & 90.48 & 93.88 & 94.03 & 94.07 & 94.07 & 93.99 & 93.62 & 78.77 & 61.67 \\
\hline 19.00 & 64.52 & 90.73 & 94.12 & 94.27 & 94.31 & 94.31 & 94.23 & 93.86 & 79.02 & 61.84 \\
\hline 20.00 & 64.95 & 91.70 & 95.15 & 95.32 & 95.37 & 95.36 & 95.26 & 94.88 & 79.78 & 62.09 \\
\hline 21.00 & 65.20 & 91.94 & 95.41 & 95.59 & 95.64 & 95.64 & 95.53 & 95.12 & 80.03 & 62.30 \\
\hline 22.00 & 60.29 & 61.98 & 62.98 & 63.14 & 63.19 & 63.19 & 63.07 & 62.73 & 60.58 & 59.17 \\
\hline 22.50 & 58.62 & 57.94 & 58.10 & 58.22 & 58.28 & 58.28 & 58.16 & 57.99 & 57.92 & 58.04 \\
\hline
\end{tabular}


Table B - 39. Temperature $\left({ }^{\circ} \mathrm{C}\right)$ map of the east side of plate $2 \mathrm{BZ}$ at MOC 142B (96.4 EFPD).

\begin{tabular}{|c||c|c|c|c|c|c|c|c|c|c|}
\hline \multirow{2}{*}{$\begin{array}{c}\text { Length of } \\
\text { Plate (in) }\end{array}$} & \multicolumn{10}{|c|}{ Width of Plate (inches) } \\
\hline 22.625 & 57.68 & 56.81 & 56.95 & 57.06 & 57.12 & 57.11 & 57.01 & 56.86 & 56.83 & 57.58 \\
\hline 23.00 & 58.73 & 59.93 & 60.71 & 60.84 & 60.89 & 60.89 & 60.78 & 60.51 & 58.91 & 58.39 \\
\hline 24.00 & 62.14 & 83.29 & 86.06 & 86.20 & 86.24 & 86.24 & 86.15 & 85.83 & 74.09 & 60.80 \\
\hline 25.00 & 62.33 & 83.49 & 86.26 & 86.40 & 86.45 & 86.44 & 86.36 & 86.02 & 74.30 & 60.99 \\
\hline 26.00 & 62.48 & 83.70 & 86.48 & 86.63 & 86.67 & 86.67 & 86.58 & 86.24 & 74.51 & 61.19 \\
\hline 27.00 & 62.63 & 83.85 & 86.63 & 86.78 & 86.82 & 86.82 & 86.73 & 86.39 & 74.68 & 61.37 \\
\hline 28.00 & 62.78 & 84.07 & 86.85 & 87.00 & 87.05 & 87.05 & 86.95 & 86.62 & 74.88 & 61.48 \\
\hline 29.00 & 62.92 & 84.23 & 87.01 & 87.17 & 87.21 & 87.21 & 87.11 & 86.78 & 75.04 & 61.57 \\
\hline 30.00 & 63.02 & 84.30 & 87.07 & 87.23 & 87.28 & 87.27 & 87.17 & 86.83 & 75.14 & 61.67 \\
\hline 31.00 & 63.13 & 84.43 & 87.20 & 87.36 & 87.41 & 87.41 & 87.31 & 86.97 & 75.29 & 61.81 \\
\hline 32.00 & 63.12 & 83.82 & 86.53 & 86.69 & 86.74 & 86.74 & 86.63 & 86.29 & 74.93 & 61.83 \\
\hline 33.00 & 63.22 & 83.97 & 86.68 & 86.84 & 86.89 & 86.89 & 86.78 & 86.44 & 75.08 & 61.90 \\
\hline 34.00 & 63.26 & 83.91 & 86.60 & 86.77 & 86.82 & 86.82 & 86.71 & 86.37 & 75.08 & 61.94 \\
\hline 35.00 & 63.34 & 84.04 & 86.73 & 86.90 & 86.95 & 86.95 & 86.84 & 86.50 & 75.22 & 62.07 \\
\hline 36.00 & 63.23 & 83.18 & 85.79 & 85.96 & 86.02 & 86.01 & 85.90 & 85.56 & 74.70 & 62.03 \\
\hline 37.00 & 63.29 & 83.32 & 85.93 & 86.10 & 86.15 & 86.15 & 86.04 & 85.70 & 74.82 & 62.05 \\
\hline 38.00 & 63.20 & 82.72 & 85.28 & 85.45 & 85.51 & 85.50 & 85.39 & 85.05 & 74.45 & 61.95 \\
\hline 39.00 & 63.23 & 82.83 & 85.39 & 85.56 & 85.62 & 85.62 & 85.50 & 85.16 & 74.56 & 61.97 \\
\hline 40.00 & 62.95 & 80.99 & 83.38 & 83.55 & 83.61 & 83.61 & 83.49 & 83.16 & 73.37 & 61.80 \\
\hline 41.00 & 62.95 & 81.08 & 83.47 & 83.65 & 83.71 & 83.70 & 83.58 & 83.25 & 73.45 & 61.80 \\
\hline 42.00 & 62.65 & 79.39 & 81.63 & 81.80 & 81.86 & 81.85 & 81.73 & 81.41 & 72.35 & 61.55 \\
\hline 43.00 & 62.62 & 79.47 & 81.70 & 81.87 & 81.93 & 81.93 & 81.81 & 81.48 & 72.42 & 61.52 \\
\hline 44.00 & 59.98 & 60.71 & 61.08 & 61.25 & 61.33 & 61.32 & 61.17 & 60.94 & 60.40 & 59.74 \\
\hline 45.00 & 59.47 & 60.30 & 60.56 & 60.71 & 60.79 & 60.78 & 60.64 & 60.45 & 60.06 & 59.33 \\
\hline
\end{tabular}


Table B - 40. Temperature $\left({ }^{\circ} \mathrm{C}\right)$ map of the west side of plate 2BZ at MOC 142B (96.4 EFPD).

\begin{tabular}{|c||c|c|c|c|c|c|c|c|c|c|}
\hline \multirow{2}{*}{$\begin{array}{c}\text { Length of } \\
\text { Plate (in) }\end{array}$} & \multicolumn{10}{|c|}{ Width of Plate (inches) } \\
\hline 22.625 & 58.73 & 57.98 & 0.50 & 0.75 & 1.00 & 1.25 & 1.50 & 1.75 & 2.00 & 2.24 \\
\hline 23.00 & 60.12 & 61.10 & 61.89 & 62.04 & 62.10 & 62.09 & 61.97 & 61.68 & 60.04 & 59.08 \\
\hline 24.00 & 64.45 & 84.75 & 87.54 & 87.70 & 87.75 & 87.74 & 87.64 & 87.29 & 75.36 & 61.97 \\
\hline 25.00 & 64.68 & 85.03 & 87.81 & 87.98 & 88.03 & 88.03 & 87.92 & 87.57 & 75.63 & 62.18 \\
\hline 26.00 & 64.84 & 85.32 & 88.11 & 88.28 & 88.34 & 88.33 & 88.22 & 87.86 & 75.92 & 62.44 \\
\hline 27.00 & 65.02 & 85.56 & 88.35 & 88.52 & 88.58 & 88.57 & 88.46 & 88.10 & 76.16 & 62.66 \\
\hline 28.00 & 65.20 & 85.86 & 88.66 & 88.83 & 88.89 & 88.89 & 88.77 & 88.40 & 76.43 & 62.75 \\
\hline 29.00 & 65.37 & 86.11 & 88.91 & 89.09 & 89.15 & 89.15 & 89.03 & 88.66 & 76.68 & 62.81 \\
\hline 30.00 & 65.47 & 86.26 & 89.06 & 89.24 & 89.30 & 89.30 & 89.17 & 88.80 & 76.85 & 62.90 \\
\hline 31.00 & 65.60 & 86.48 & 89.28 & 89.47 & 89.53 & 89.52 & 89.39 & 89.02 & 77.07 & 63.06 \\
\hline 32.00 & 65.58 & 85.96 & 88.70 & 88.88 & 88.95 & 88.94 & 88.81 & 88.44 & 76.79 & 63.07 \\
\hline 33.00 & 65.71 & 86.20 & 88.94 & 89.13 & 89.20 & 89.19 & 89.05 & 88.68 & 77.02 & 63.13 \\
\hline 34.00 & 65.73 & 86.23 & 88.95 & 89.14 & 89.21 & 89.21 & 89.07 & 88.69 & 77.10 & 63.17 \\
\hline 35.00 & 65.83 & 86.43 & 89.16 & 89.35 & 89.42 & 89.42 & 89.28 & 88.90 & 77.31 & 63.35 \\
\hline 36.00 & 65.64 & 85.66 & 88.31 & 88.51 & 88.58 & 88.57 & 88.43 & 88.05 & 76.85 & 63.30 \\
\hline 37.00 & 65.72 & 85.88 & 88.53 & 88.73 & 88.80 & 88.79 & 88.65 & 88.27 & 77.05 & 63.30 \\
\hline 38.00 & 65.57 & 85.36 & 87.96 & 88.16 & 88.23 & 88.23 & 88.08 & 87.70 & 76.74 & 63.11 \\
\hline 39.00 & 65.61 & 85.54 & 88.14 & 88.34 & 88.41 & 88.41 & 88.26 & 87.87 & 76.91 & 63.13 \\
\hline 40.00 & 65.22 & 83.79 & 86.22 & 86.43 & 86.50 & 86.49 & 86.34 & 85.96 & 75.79 & 62.92 \\
\hline 41.00 & 65.22 & 83.94 & 86.37 & 86.58 & 86.65 & 86.65 & 86.49 & 86.11 & 75.93 & 62.92 \\
\hline 42.00 & 64.82 & 82.34 & 84.62 & 84.82 & 84.90 & 84.89 & 84.74 & 84.36 & 74.90 & 62.59 \\
\hline 43.00 & 64.78 & 82.46 & 84.74 & 84.95 & 85.02 & 85.02 & 84.86 & 84.49 & 75.01 & 62.57 \\
\hline 44.00 & 61.57 & 63.19 & 63.61 & 63.80 & 63.89 & 63.88 & 63.70 & 63.42 & 62.80 & 60.52 \\
\hline 45.00 & 60.94 & 62.79 & 63.09 & 63.27 & 63.36 & 63.35 & 63.18 & 62.95 & 62.47 & 60.07 \\
\hline
\end{tabular}


Table B - 41. Temperature $\left({ }^{\circ} \mathrm{C}\right)$ map of the east side of plate 2TT at MOC 142B (110.1 EFPD).

\begin{tabular}{|c||c|c|c|c|c|c|c|c|c|c|}
\hline \multirow{2}{*}{$\begin{array}{c}\text { Length of } \\
\text { Plate (in) }\end{array}$} & \multicolumn{10}{|c|}{ Width of Plate (inches) } \\
\hline 0.125 & 51.94 & 51.78 & 51.77 & 51.77 & 51.77 & 51.77 & 51.77 & 51.77 & 51.81 & 51.95 \\
\hline 1.00 & 52.98 & 52.43 & 52.57 & 52.59 & 52.59 & 52.59 & 52.59 & 52.52 & 52.39 & 52.90 \\
\hline 2.00 & 56.65 & 78.38 & 81.08 & 81.15 & 81.15 & 81.15 & 81.14 & 80.90 & 69.08 & 55.42 \\
\hline 3.00 & 57.01 & 78.62 & 81.35 & 81.42 & 81.42 & 81.42 & 81.41 & 81.15 & 69.35 & 55.76 \\
\hline 4.00 & 57.83 & 82.62 & 85.67 & 85.74 & 85.75 & 85.75 & 85.73 & 85.46 & 72.11 & 56.41 \\
\hline 5.00 & 58.11 & 82.79 & 85.84 & 85.93 & 85.93 & 85.93 & 85.91 & 85.63 & 72.31 & 56.68 \\
\hline 6.00 & 58.90 & 86.18 & 89.50 & 89.59 & 89.60 & 89.59 & 89.57 & 89.27 & 74.69 & 57.30 \\
\hline 7.00 & 59.21 & 86.38 & 89.69 & 89.79 & 89.80 & 89.80 & 89.77 & 89.46 & 74.91 & 57.58 \\
\hline 8.00 & 59.77 & 88.22 & 91.67 & 91.77 & 91.78 & 91.78 & 91.75 & 91.43 & 76.25 & 58.05 \\
\hline 9.00 & 60.03 & 88.39 & 91.84 & 91.94 & 91.96 & 91.95 & 91.92 & 91.60 & 76.47 & 58.39 \\
\hline 10.00 & 60.51 & 89.72 & 93.26 & 93.37 & 93.38 & 93.38 & 93.35 & 93.01 & 77.47 & 58.79 \\
\hline 11.00 & 60.81 & 89.92 & 93.46 & 93.57 & 93.59 & 93.58 & 93.54 & 93.21 & 77.69 & 59.04 \\
\hline 12.00 & 61.21 & 90.85 & 94.44 & 94.56 & 94.58 & 94.58 & 94.53 & 94.19 & 78.41 & 59.39 \\
\hline 13.00 & 61.46 & 91.03 & 94.62 & 94.74 & 94.76 & 94.76 & 94.71 & 94.36 & 78.62 & 59.67 \\
\hline 14.00 & 61.74 & 91.21 & 94.80 & 94.92 & 94.95 & 94.95 & 94.90 & 94.54 & 78.84 & 59.95 \\
\hline 15.00 & 61.99 & 91.39 & 94.97 & 95.10 & 95.13 & 95.13 & 95.07 & 94.71 & 79.03 & 60.16 \\
\hline 16.00 & 62.32 & 91.95 & 95.56 & 95.70 & 95.73 & 95.73 & 95.66 & 95.30 & 79.51 & 60.44 \\
\hline 17.00 & 62.56 & 92.13 & 95.75 & 95.89 & 95.92 & 95.92 & 95.85 & 95.48 & 79.73 & 60.76 \\
\hline 18.00 & 62.77 & 92.02 & 95.62 & 95.76 & 95.79 & 95.79 & 95.72 & 95.34 & 79.75 & 61.01 \\
\hline 19.00 & 63.00 & 92.20 & 95.79 & 95.94 & 95.98 & 95.97 & 95.90 & 95.52 & 79.94 & 61.21 \\
\hline 20.00 & 63.32 & 92.82 & 96.45 & 96.60 & 96.64 & 96.64 & 96.56 & 96.17 & 80.44 & 61.45 \\
\hline 21.00 & 63.52 & 92.98 & 96.62 & 96.77 & 96.81 & 96.81 & 96.73 & 96.33 & 80.63 & 61.65 \\
\hline 22.00 & 59.28 & 61.53 & 62.59 & 62.74 & 62.80 & 62.79 & 62.68 & 62.34 & 60.05 & 58.82 \\
\hline 22.50 & 57.86 & 57.21 & 57.38 & 57.50 & 57.56 & 57.55 & 57.44 & 57.28 & 57.20 & 57.74 \\
\hline
\end{tabular}


Table B - 42. Temperature $\left({ }^{\circ} \mathrm{C}\right)$ map of the west side of plate 2TT at MOC 142B (110.1 EFPD).

\begin{tabular}{|c||c|c|c|c|c|c|c|c|c|c|}
\hline \multirow{2}{*}{$\begin{array}{c}\text { Length of } \\
\text { Plate (in) }\end{array}$} & \multicolumn{10}{|c|}{ Width of Plate (inches) } \\
\hline & 0.05 & 0.25 & 0.50 & 0.75 & 1.00 & 1.25 & 1.50 & 1.75 & 2.00 & 2.24 \\
\hline 0.125 & 51.98 & 51.78 & 51.77 & 51.77 & 51.77 & 51.77 & 51.77 & 51.77 & 51.81 & 51.98 \\
\hline 1.00 & 53.22 & 52.43 & 52.58 & 52.59 & 52.60 & 52.60 & 52.59 & 52.52 & 52.40 & 53.04 \\
\hline 2.00 & 57.55 & 78.40 & 81.10 & 81.17 & 81.17 & 81.17 & 81.16 & 80.92 & 69.08 & 55.87 \\
\hline 3.00 & 57.97 & 78.67 & 81.39 & 81.47 & 81.47 & 81.47 & 81.46 & 81.20 & 69.38 & 56.23 \\
\hline 4.00 & 58.96 & 82.70 & 85.75 & 85.83 & 85.84 & 85.84 & 85.82 & 85.54 & 72.17 & 56.99 \\
\hline 5.00 & 59.29 & 82.92 & 85.97 & 86.06 & 86.07 & 86.06 & 86.04 & 85.76 & 72.41 & 57.28 \\
\hline 6.00 & 60.28 & 86.36 & 89.67 & 89.76 & 89.78 & 89.77 & 89.75 & 89.45 & 74.83 & 57.98 \\
\hline 7.00 & 60.64 & 86.62 & 89.93 & 90.03 & 90.04 & 90.04 & 90.01 & 89.70 & 75.10 & 58.28 \\
\hline 8.00 & 61.35 & 88.52 & 91.97 & 92.07 & 92.08 & 92.08 & 92.05 & 91.72 & 76.50 & 58.82 \\
\hline 9.00 & 61.66 & 88.76 & 92.20 & 92.31 & 92.33 & 92.33 & 92.29 & 91.96 & 76.77 & 59.27 \\
\hline 10.00 & 62.29 & 90.16 & 93.69 & 93.80 & 93.82 & 93.82 & 93.78 & 93.44 & 77.83 & 59.73 \\
\hline 11.00 & 62.63 & 90.43 & 93.97 & 94.09 & 94.11 & 94.11 & 94.06 & 93.71 & 78.12 & 59.96 \\
\hline 12.00 & 63.14 & 91.44 & 95.03 & 95.15 & 95.18 & 95.18 & 95.12 & 94.77 & 78.91 & 60.36 \\
\hline 13.00 & 63.44 & 91.69 & 95.28 & 95.41 & 95.44 & 95.44 & 95.38 & 95.02 & 79.20 & 60.67 \\
\hline 14.00 & 63.80 & 91.96 & 95.54 & 95.68 & 95.71 & 95.71 & 95.64 & 95.28 & 79.48 & 61.00 \\
\hline 15.00 & 64.10 & 92.22 & 95.80 & 95.94 & 95.98 & 95.97 & 95.90 & 95.53 & 79.75 & 61.22 \\
\hline 16.00 & 64.51 & 92.87 & 96.50 & 96.66 & 96.70 & 96.70 & 96.61 & 96.21 & 80.31 & 61.52 \\
\hline 17.00 & 64.80 & 93.14 & 96.79 & 96.96 & 97.01 & 97.00 & 96.91 & 96.50 & 80.61 & 61.93 \\
\hline 18.00 & 65.06 & 93.12 & 96.77 & 96.94 & 96.99 & 96.99 & 96.89 & 96.47 & 80.71 & 62.26 \\
\hline 19.00 & 65.34 & 93.38 & 97.06 & 97.24 & 97.29 & 97.29 & 97.18 & 96.76 & 80.98 & 62.45 \\
\hline 20.00 & 65.74 & 94.10 & 97.87 & 98.05 & 98.11 & 98.10 & 97.99 & 97.56 & 81.57 & 62.67 \\
\hline 21.00 & 65.99 & 94.34 & 98.14 & 98.34 & 98.39 & 98.39 & 98.27 & 97.84 & 81.83 & 62.88 \\
\hline 22.00 & 60.76 & 62.78 & 63.86 & 64.03 & 64.09 & 64.09 & 63.95 & 63.59 & 61.27 & 59.55 \\
\hline 22.50 & 58.99 & 58.52 & 58.70 & 58.84 & 58.91 & 58.90 & 58.77 & 58.58 & 58.46 & 58.34 \\
\hline
\end{tabular}


Table B - 43. Temperature $\left({ }^{\circ} \mathrm{C}\right)$ map of the east side of plate 2BZ at MOC 142B (110.1 EFPD).

\begin{tabular}{|c||c|c|c|c|c|c|c|c|c|c|}
\hline \multirow{2}{*}{$\begin{array}{l}\text { Length of } \\
\text { Plate (in) }\end{array}$} & \multicolumn{10}{|c|}{ Width of Plate (inches) } \\
\hline 22.625 & 57.98 & 57.25 & 57.41 & 57.54 & 57.60 & 57.59 & 57.48 & 57.31 & 57.23 & 57.85 \\
\hline 23.00 & 59.09 & 60.54 & 61.38 & 61.52 & 61.58 & 61.57 & 61.46 & 61.16 & 59.44 & 58.71 \\
\hline 24.00 & 62.70 & 85.14 & 88.04 & 88.19 & 88.23 & 88.23 & 88.14 & 87.80 & 75.46 & 61.27 \\
\hline 25.00 & 62.92 & 85.38 & 88.29 & 88.44 & 88.49 & 88.49 & 88.39 & 88.04 & 75.70 & 61.47 \\
\hline 26.00 & 63.12 & 85.94 & 88.88 & 89.04 & 89.09 & 89.08 & 88.99 & 88.64 & 76.16 & 61.71 \\
\hline 27.00 & 63.28 & 86.10 & 89.04 & 89.20 & 89.25 & 89.25 & 89.15 & 88.79 & 76.34 & 61.90 \\
\hline 28.00 & 63.44 & 86.35 & 89.30 & 89.46 & 89.51 & 89.51 & 89.41 & 89.05 & 76.57 & 62.03 \\
\hline 29.00 & 63.60 & 86.52 & 89.47 & 89.64 & 89.69 & 89.69 & 89.58 & 89.22 & 76.74 & 62.13 \\
\hline 30.00 & 63.73 & 86.68 & 89.63 & 89.80 & 89.86 & 89.85 & 89.74 & 89.38 & 76.90 & 62.25 \\
\hline 31.00 & 63.85 & 86.83 & 89.78 & 89.96 & 90.01 & 90.01 & 89.90 & 89.53 & 77.07 & 62.40 \\
\hline 32.00 & 63.84 & 86.20 & 89.09 & 89.27 & 89.33 & 89.32 & 89.21 & 88.84 & 76.70 & 62.42 \\
\hline 33.00 & 63.95 & 86.37 & 89.26 & 89.44 & 89.49 & 89.49 & 89.37 & 89.01 & 76.86 & 62.50 \\
\hline 34.00 & 64.02 & 86.44 & 89.33 & 89.51 & 89.57 & 89.57 & 89.45 & 89.08 & 76.96 & 62.56 \\
\hline 35.00 & 64.11 & 86.58 & 89.48 & 89.66 & 89.72 & 89.71 & 89.60 & 89.22 & 77.11 & 62.70 \\
\hline 36.00 & 64.08 & 86.18 & 89.03 & 89.22 & 89.28 & 89.27 & 89.15 & 88.78 & 76.89 & 62.73 \\
\hline 37.00 & 64.16 & 86.33 & 89.19 & 89.37 & 89.43 & 89.43 & 89.31 & 88.93 & 77.04 & 62.76 \\
\hline 38.00 & 64.06 & 85.57 & 88.35 & 88.54 & 88.60 & 88.60 & 88.47 & 88.10 & 76.55 & 62.65 \\
\hline 39.00 & 64.11 & 85.70 & 88.48 & 88.67 & 88.73 & 88.73 & 88.60 & 88.23 & 76.68 & 62.69 \\
\hline 40.00 & 63.87 & 84.21 & 86.86 & 87.04 & 87.11 & 87.10 & 86.98 & 86.61 & 75.72 & 62.55 \\
\hline 41.00 & 63.88 & 84.32 & 86.97 & 87.15 & 87.22 & 87.21 & 87.09 & 86.72 & 75.83 & 62.56 \\
\hline 42.00 & 63.54 & 82.27 & 84.72 & 84.91 & 84.98 & 84.97 & 84.84 & 84.49 & 74.49 & 62.29 \\
\hline 43.00 & 63.51 & 82.35 & 84.81 & 85.00 & 85.07 & 85.06 & 84.93 & 84.57 & 74.56 & 62.26 \\
\hline 44.00 & 60.57 & 61.54 & 61.96 & 62.15 & 62.23 & 62.22 & 62.06 & 61.80 & 61.17 & 60.28 \\
\hline 45.00 & 60.01 & 61.09 & 61.38 & 61.55 & 61.63 & 61.63 & 61.47 & 61.26 & 60.80 & 59.82 \\
\hline
\end{tabular}


Table B - 44. Temperature $\left({ }^{\circ} \mathrm{C}\right)$ map of the west side of plate 2BZ at MOC 142B (110.1 EFPD).

\begin{tabular}{|c||c|c|c|c|c|c|c|c|c|c|}
\hline \multirow{2}{*}{$\begin{array}{c}\text { Length of } \\
\text { Plate (in) }\end{array}$} & \multicolumn{10}{|c|}{ Width of Plate (inches) } \\
\hline 22.625 & 59.10 & 58.56 & 58.75 & 58.89 & 58.95 & 58.95 & 58.82 & 58.63 & 58.50 & 58.43 \\
\hline 23.00 & 60.58 & 61.85 & 62.70 & 62.87 & 62.93 & 62.92 & 62.79 & 62.47 & 60.70 & 59.45 \\
\hline 24.00 & 65.19 & 86.76 & 89.68 & 89.85 & 89.91 & 89.91 & 89.79 & 89.42 & 76.87 & 62.53 \\
\hline 25.00 & 65.44 & 87.09 & 90.02 & 90.20 & 90.25 & 90.25 & 90.13 & 89.75 & 77.19 & 62.76 \\
\hline 26.00 & 65.66 & 87.73 & 90.69 & 90.88 & 90.94 & 90.93 & 90.81 & 90.43 & 77.72 & 63.06 \\
\hline 27.00 & 65.86 & 87.98 & 90.94 & 91.13 & 91.19 & 91.19 & 91.06 & 90.68 & 77.98 & 63.30 \\
\hline 28.00 & 66.06 & 88.33 & 91.29 & 91.48 & 91.55 & 91.54 & 91.41 & 91.03 & 78.29 & 63.40 \\
\hline 29.00 & 66.25 & 88.60 & 91.57 & 91.78 & 91.85 & 91.85 & 91.70 & 91.30 & 78.55 & 63.47 \\
\hline 30.00 & 66.39 & 88.85 & 91.85 & 92.06 & 92.14 & 92.13 & 91.98 & 91.56 & 78.80 & 63.59 \\
\hline 31.00 & 66.54 & 89.09 & 92.11 & 92.33 & 92.40 & 92.40 & 92.24 & 91.82 & 79.05 & 63.77 \\
\hline 32.00 & 66.51 & 88.57 & 91.52 & 91.74 & 91.81 & 91.81 & 91.65 & 91.23 & 78.77 & 63.77 \\
\hline 33.00 & 66.65 & 88.83 & 91.81 & 92.03 & 92.11 & 92.10 & 91.94 & 91.51 & 79.02 & 63.84 \\
\hline 34.00 & 66.70 & 88.99 & 91.99 & 92.21 & 92.29 & 92.29 & 92.12 & 91.69 & 79.19 & 63.90 \\
\hline 35.00 & 66.81 & 89.22 & 92.23 & 92.46 & 92.54 & 92.54 & 92.37 & 91.94 & 79.43 & 64.10 \\
\hline 36.00 & 66.74 & 88.91 & 91.88 & 92.11 & 92.20 & 92.19 & 92.02 & 91.58 & 79.28 & 64.13 \\
\hline 37.00 & 66.83 & 89.15 & 92.14 & 92.38 & 92.46 & 92.46 & 92.28 & 91.84 & 79.51 & 64.14 \\
\hline 38.00 & 66.67 & 88.48 & 91.39 & 91.62 & 91.71 & 91.70 & 91.53 & 91.09 & 79.10 & 63.94 \\
\hline 39.00 & 66.73 & 88.68 & 91.61 & 91.84 & 91.93 & 91.92 & 91.74 & 91.30 & 79.29 & 63.97 \\
\hline 40.00 & 66.39 & 87.29 & 90.04 & 90.28 & 90.37 & 90.36 & 90.18 & 89.74 & 78.41 & 63.79 \\
\hline 41.00 & 66.41 & 87.46 & 90.23 & 90.47 & 90.56 & 90.55 & 90.37 & 89.93 & 78.58 & 63.81 \\
\hline 42.00 & 65.95 & 85.52 & 88.05 & 88.29 & 88.38 & 88.37 & 88.18 & 87.76 & 77.32 & 63.44 \\
\hline 43.00 & 65.92 & 85.66 & 88.20 & 88.44 & 88.53 & 88.52 & 88.33 & 87.91 & 77.44 & 63.42 \\
\hline 44.00 & 62.33 & 64.31 & 64.79 & 65.01 & 65.10 & 65.09 & 64.89 & 64.58 & 63.86 & 61.14 \\
\hline 45.00 & 61.64 & 63.87 & 64.22 & 64.43 & 64.52 & 64.51 & 64.32 & 64.06 & 63.50 & 60.64 \\
\hline
\end{tabular}


Table B - 45. Temperature $\left({ }^{\circ} \mathrm{C}\right)$ map of the east side of plate 2TT at EOC 142B (132.4 EFPD).

\begin{tabular}{|c||c|c|c|c|c|c|c|c|c|c|}
\hline \multirow{2}{*}{$\begin{array}{c}\text { Length of } \\
\text { Plate (in) }\end{array}$} & \multicolumn{9}{|c|}{ Width of Plate (inches) } \\
\hline & 0.05 & 0.25 & 0.50 & 0.75 & 1.00 & 1.25 & 1.50 & 1.75 & 2.00 & 2.24 \\
\hline 0.125 & 51.94 & 51.78 & 51.77 & 51.77 & 51.77 & 51.77 & 51.77 & 51.77 & 51.81 & 51.95 \\
\hline 1.00 & 52.95 & 52.39 & 52.53 & 52.54 & 52.55 & 52.55 & 52.54 & 52.48 & 52.37 & 52.87 \\
\hline 2.00 & 56.41 & 76.93 & 79.51 & 79.58 & 79.58 & 79.58 & 79.57 & 79.34 & 68.11 & 55.25 \\
\hline 3.00 & 56.74 & 77.16 & 79.76 & 79.82 & 79.83 & 79.83 & 79.82 & 79.57 & 68.36 & 55.57 \\
\hline 4.00 & 57.45 & 80.42 & 83.28 & 83.36 & 83.36 & 83.36 & 83.35 & 83.08 & 70.61 & 56.14 \\
\hline 5.00 & 57.71 & 80.58 & 83.45 & 83.52 & 83.53 & 83.53 & 83.51 & 83.24 & 70.80 & 56.40 \\
\hline 6.00 & 58.35 & 83.09 & 86.16 & 86.24 & 86.25 & 86.25 & 86.23 & 85.94 & 72.57 & 56.90 \\
\hline 7.00 & 58.63 & 83.27 & 86.34 & 86.42 & 86.43 & 86.43 & 86.41 & 86.11 & 72.77 & 57.16 \\
\hline 8.00 & 59.06 & 84.43 & 87.58 & 87.67 & 87.68 & 87.68 & 87.66 & 87.35 & 73.64 & 57.54 \\
\hline 9.00 & 59.31 & 84.59 & 87.74 & 87.83 & 87.84 & 87.84 & 87.81 & 87.51 & 73.84 & 57.86 \\
\hline 10.00 & 59.67 & 85.32 & 88.51 & 88.61 & 88.63 & 88.63 & 88.59 & 88.28 & 74.41 & 58.17 \\
\hline 11.00 & 59.95 & 85.50 & 88.69 & 88.79 & 88.81 & 88.81 & 88.77 & 88.45 & 74.61 & 58.40 \\
\hline 12.00 & 60.25 & 85.97 & 89.18 & 89.29 & 89.31 & 89.30 & 89.26 & 88.94 & 75.01 & 58.69 \\
\hline 13.00 & 60.48 & 86.13 & 89.34 & 89.45 & 89.47 & 89.47 & 89.42 & 89.10 & 75.20 & 58.94 \\
\hline 14.00 & 60.69 & 86.02 & 89.20 & 89.31 & 89.34 & 89.34 & 89.29 & 88.96 & 75.20 & 59.17 \\
\hline 15.00 & 60.92 & 86.18 & 89.36 & 89.47 & 89.50 & 89.50 & 89.44 & 89.11 & 75.38 & 59.36 \\
\hline 16.00 & 61.16 & 86.33 & 89.49 & 89.62 & 89.64 & 89.64 & 89.59 & 89.25 & 75.55 & 59.58 \\
\hline 17.00 & 61.38 & 86.48 & 89.65 & 89.77 & 89.80 & 89.80 & 89.74 & 89.41 & 75.75 & 59.87 \\
\hline 18.00 & 61.58 & 86.49 & 89.64 & 89.76 & 89.80 & 89.79 & 89.73 & 89.39 & 75.84 & 60.11 \\
\hline 19.00 & 61.78 & 86.64 & 89.79 & 89.92 & 89.95 & 89.95 & 89.88 & 89.54 & 76.01 & 60.29 \\
\hline 20.00 & 62.01 & 86.74 & 89.87 & 90.01 & 90.04 & 90.04 & 89.97 & 89.62 & 76.14 & 60.46 \\
\hline 21.00 & 62.18 & 86.87 & 90.00 & 90.14 & 90.18 & 90.18 & 90.10 & 89.76 & 76.30 & 60.62 \\
\hline 22.00 & 58.63 & 60.20 & 61.07 & 61.21 & 61.25 & 61.25 & 61.15 & 60.86 & 59.00 & 58.26 \\
\hline 22.50 & 57.45 & 56.58 & 56.70 & 56.80 & 56.85 & 56.85 & 56.75 & 56.62 & 56.62 & 57.37 \\
\hline
\end{tabular}


Table B - 46. Temperature $\left({ }^{\circ} \mathrm{C}\right)$ map of the west side of plate 2TT at EOC 142B (132.4 EFPD).

\begin{tabular}{|c||c|c|c|c|c|c|c|c|c|c|}
\hline \multirow{2}{*}{$\begin{array}{c}\text { Length of } \\
\text { Plate (in) }\end{array}$} & 0.05 & 0.25 & 0.50 & 0.75 & 1.00 & 1.25 & 1.50 & 1.75 & 2.00 & 2.24 \\
\hline 0.125 & 51.98 & 51.78 & 51.77 & 51.77 & 51.77 & 51.77 & 51.77 & 51.77 & 51.81 & 51.98 \\
\hline 1.00 & 53.18 & 52.40 & 52.53 & 52.55 & 52.55 & 52.55 & 52.54 & 52.48 & 52.37 & 53.00 \\
\hline 2.00 & 57.26 & 76.95 & 79.53 & 79.60 & 79.60 & 79.60 & 79.59 & 79.35 & 68.11 & 55.68 \\
\hline 3.00 & 57.66 & 77.21 & 79.80 & 79.87 & 79.88 & 79.88 & 79.86 & 79.61 & 68.39 & 56.02 \\
\hline 4.00 & 58.52 & 80.50 & 83.36 & 83.44 & 83.45 & 83.45 & 83.43 & 83.16 & 70.67 & 56.69 \\
\hline 5.00 & 58.83 & 80.71 & 83.57 & 83.65 & 83.66 & 83.66 & 83.64 & 83.36 & 70.89 & 56.96 \\
\hline 6.00 & 59.62 & 83.26 & 86.32 & 86.41 & 86.42 & 86.42 & 86.40 & 86.11 & 72.70 & 57.53 \\
\hline 7.00 & 59.96 & 83.50 & 86.56 & 86.65 & 86.66 & 86.66 & 86.63 & 86.34 & 72.95 & 57.80 \\
\hline 8.00 & 60.52 & 84.72 & 87.86 & 87.95 & 87.97 & 87.97 & 87.94 & 87.63 & 73.87 & 58.25 \\
\hline 9.00 & 60.81 & 84.93 & 88.07 & 88.17 & 88.19 & 88.19 & 88.15 & 87.84 & 74.12 & 58.67 \\
\hline 10.00 & 61.28 & 85.73 & 88.91 & 89.02 & 89.04 & 89.04 & 88.99 & 88.68 & 74.75 & 59.03 \\
\hline 11.00 & 61.60 & 85.98 & 89.16 & 89.27 & 89.29 & 89.29 & 89.25 & 88.92 & 75.01 & 59.24 \\
\hline 12.00 & 61.99 & 86.51 & 89.72 & 89.83 & 89.86 & 89.86 & 89.81 & 89.48 & 75.47 & 59.56 \\
\hline 13.00 & 62.26 & 86.74 & 89.94 & 90.06 & 90.09 & 90.09 & 90.03 & 89.70 & 75.72 & 59.85 \\
\hline 14.00 & 62.54 & 86.70 & 89.88 & 90.01 & 90.04 & 90.04 & 89.97 & 89.64 & 75.79 & 60.11 \\
\hline 15.00 & 62.81 & 86.93 & 90.11 & 90.24 & 90.27 & 90.27 & 90.20 & 89.86 & 76.02 & 60.30 \\
\hline 16.00 & 63.11 & 87.16 & 90.33 & 90.47 & 90.50 & 90.50 & 90.43 & 90.08 & 76.28 & 60.53 \\
\hline 17.00 & 63.36 & 87.40 & 90.56 & 90.70 & 90.74 & 90.74 & 90.66 & 90.31 & 76.54 & 60.92 \\
\hline 18.00 & 63.62 & 87.47 & 90.63 & 90.77 & 90.81 & 90.81 & 90.73 & 90.37 & 76.69 & 61.22 \\
\hline 19.00 & 63.86 & 87.71 & 90.86 & 91.00 & 91.04 & 91.04 & 90.96 & 90.60 & 76.93 & 61.39 \\
\hline 20.00 & 64.14 & 87.89 & 91.03 & 91.18 & 91.22 & 91.22 & 91.13 & 90.77 & 77.14 & 61.53 \\
\hline 21.00 & 64.34 & 88.10 & 91.24 & 91.39 & 91.44 & 91.43 & 91.34 & 90.98 & 77.36 & 61.70 \\
\hline 22.00 & 59.96 & 61.29 & 62.18 & 62.32 & 62.38 & 62.38 & 62.26 & 61.95 & 60.06 & 58.91 \\
\hline 22.50 & 58.47 & 57.72 & 57.85 & 57.97 & 58.03 & 58.02 & 57.91 & 57.75 & 57.71 & 57.92 \\
\hline
\end{tabular}


Table B - 47. Temperature $\left({ }^{\circ} \mathrm{C}\right)$ map of the east side of plate $2 \mathrm{BZ}$ at EOC 142B (132.4 EFPD).

\begin{tabular}{|c||c|c|c|c|c|c|c|c|c|c|}
\hline \multirow{2}{*}{$\begin{array}{c}\text { Length of } \\
\text { Plate (in) }\end{array}$} & \multicolumn{10}{|c|}{ Width of Plate (inches) } \\
\hline 22.625 & 57.55 & 56.61 & 56.73 & 56.84 & 56.89 & 56.88 & 56.78 & 56.65 & 56.64 & 57.46 \\
\hline 23.00 & 58.47 & 59.35 & 60.03 & 60.16 & 60.21 & 60.20 & 60.10 & 59.85 & 58.47 & 58.18 \\
\hline 24.00 & 61.49 & 80.00 & 82.49 & 82.63 & 82.67 & 82.67 & 82.58 & 82.28 & 71.83 & 60.32 \\
\hline 25.00 & 61.68 & 80.23 & 82.73 & 82.87 & 82.91 & 82.91 & 82.82 & 82.51 & 72.06 & 60.51 \\
\hline 26.00 & 61.89 & 80.90 & 83.44 & 83.58 & 83.62 & 83.62 & 83.53 & 83.22 & 72.57 & 60.74 \\
\hline 27.00 & 62.03 & 81.04 & 83.58 & 83.72 & 83.77 & 83.76 & 83.67 & 83.36 & 72.73 & 60.91 \\
\hline 28.00 & 62.18 & 81.36 & 83.91 & 84.05 & 84.10 & 84.10 & 84.01 & 83.69 & 72.98 & 61.02 \\
\hline 29.00 & 62.32 & 81.51 & 84.06 & 84.21 & 84.25 & 84.25 & 84.16 & 83.84 & 73.13 & 61.11 \\
\hline 30.00 & 62.42 & 81.62 & 84.17 & 84.32 & 84.37 & 84.37 & 84.27 & 83.95 & 73.25 & 61.20 \\
\hline 31.00 & 62.53 & 81.77 & 84.32 & 84.47 & 84.52 & 84.51 & 84.42 & 84.10 & 73.41 & 61.35 \\
\hline 32.00 & 62.57 & 81.58 & 84.11 & 84.26 & 84.31 & 84.30 & 84.21 & 83.88 & 73.33 & 61.40 \\
\hline 33.00 & 62.67 & 81.72 & 84.25 & 84.40 & 84.45 & 84.45 & 84.35 & 84.03 & 73.47 & 61.47 \\
\hline 34.00 & 62.75 & 81.95 & 84.49 & 84.65 & 84.70 & 84.69 & 84.59 & 84.27 & 73.67 & 61.54 \\
\hline 35.00 & 62.83 & 82.08 & 84.61 & 84.77 & 84.82 & 84.82 & 84.72 & 84.39 & 73.81 & 61.66 \\
\hline 36.00 & 62.86 & 82.10 & 84.63 & 84.79 & 84.84 & 84.84 & 84.74 & 84.41 & 73.86 & 61.73 \\
\hline 37.00 & 62.92 & 82.23 & 84.77 & 84.93 & 84.98 & 84.98 & 84.87 & 84.54 & 73.99 & 61.76 \\
\hline 38.00 & 62.89 & 81.95 & 84.45 & 84.61 & 84.67 & 84.66 & 84.56 & 84.23 & 73.82 & 61.69 \\
\hline 39.00 & 62.92 & 82.06 & 84.56 & 84.73 & 84.78 & 84.78 & 84.67 & 84.34 & 73.93 & 61.72 \\
\hline 40.00 & 62.78 & 81.16 & 83.59 & 83.75 & 83.81 & 83.80 & 83.69 & 83.37 & 73.37 & 61.64 \\
\hline 41.00 & 62.79 & 81.26 & 83.69 & 83.85 & 83.91 & 83.90 & 83.80 & 83.47 & 73.46 & 61.65 \\
\hline 42.00 & 62.53 & 79.78 & 82.07 & 82.24 & 82.29 & 82.29 & 82.18 & 81.86 & 72.50 & 61.43 \\
\hline 43.00 & 62.50 & 79.86 & 82.15 & 82.32 & 82.38 & 82.37 & 82.26 & 81.94 & 72.57 & 61.40 \\
\hline 44.00 & 59.75 & 60.35 & 60.71 & 60.87 & 60.94 & 60.94 & 60.80 & 60.57 & 60.06 & 59.54 \\
\hline 45.00 & 59.21 & 59.93 & 60.16 & 60.31 & 60.38 & 60.37 & 60.24 & 60.06 & 59.71 & 59.10 \\
\hline
\end{tabular}


Table B - 48. Temperature $\left({ }^{\circ} \mathrm{C}\right)$ map of the west side of plate $2 \mathrm{BZ}$ at EOC $142 \mathrm{~B}$ (132.4 EFPD).

\begin{tabular}{|c||c|c|c|c|c|c|c|c|c|c|}
\hline \multirow{2}{*}{$\begin{array}{c}\text { Length of } \\
\text { Plate (in) }\end{array}$} & \multicolumn{10}{|c|}{ Width of Plate (inches) } \\
\hline 22.625 & 58.57 & 57.75 & 57.89 & 58.01 & 58.06 & 58.06 & 57.94 & 57.79 & 57.74 & 57.99 \\
\hline 23.00 & 59.81 & 60.49 & 61.18 & 61.32 & 61.38 & 61.37 & 61.26 & 60.99 & 59.58 & 58.84 \\
\hline 24.00 & 63.67 & 81.45 & 83.95 & 84.10 & 84.15 & 84.15 & 84.05 & 83.72 & 73.07 & 61.42 \\
\hline 25.00 & 63.90 & 81.75 & 84.26 & 84.42 & 84.47 & 84.47 & 84.36 & 84.03 & 73.36 & 61.63 \\
\hline 26.00 & 64.13 & 82.48 & 85.04 & 85.20 & 85.25 & 85.25 & 85.14 & 84.80 & 73.93 & 61.93 \\
\hline 27.00 & 64.30 & 82.70 & 85.25 & 85.42 & 85.47 & 85.47 & 85.36 & 85.02 & 74.16 & 62.15 \\
\hline 28.00 & 64.48 & 83.09 & 85.66 & 85.83 & 85.89 & 85.88 & 85.77 & 85.42 & 74.48 & 62.24 \\
\hline 29.00 & 64.65 & 83.33 & 85.90 & 86.07 & 86.13 & 86.13 & 86.01 & 85.66 & 74.71 & 62.29 \\
\hline 30.00 & 64.77 & 83.54 & 86.11 & 86.29 & 86.35 & 86.34 & 86.22 & 85.87 & 74.92 & 62.40 \\
\hline 31.00 & 64.90 & 83.75 & 86.32 & 86.50 & 86.56 & 86.55 & 86.43 & 86.08 & 75.13 & 62.56 \\
\hline 32.00 & 64.93 & 83.64 & 86.19 & 86.37 & 86.43 & 86.43 & 86.30 & 85.95 & 75.12 & 62.59 \\
\hline 33.00 & 65.05 & 83.87 & 86.43 & 86.61 & 86.67 & 86.66 & 86.53 & 86.18 & 75.34 & 62.65 \\
\hline 34.00 & 65.12 & 84.18 & 86.74 & 86.93 & 86.99 & 86.98 & 86.85 & 86.49 & 75.60 & 62.73 \\
\hline 35.00 & 65.22 & 84.37 & 86.94 & 87.13 & 87.19 & 87.19 & 87.06 & 86.69 & 75.80 & 62.90 \\
\hline 36.00 & 65.22 & 84.47 & 87.04 & 87.22 & 87.29 & 87.28 & 87.15 & 86.78 & 75.92 & 62.98 \\
\hline 37.00 & 65.30 & 84.68 & 87.25 & 87.44 & 87.51 & 87.50 & 87.37 & 87.00 & 76.12 & 62.98 \\
\hline 38.00 & 65.22 & 84.47 & 87.01 & 87.20 & 87.27 & 87.26 & 87.12 & 86.76 & 76.01 & 62.84 \\
\hline 39.00 & 65.26 & 84.65 & 87.19 & 87.38 & 87.45 & 87.45 & 87.31 & 86.94 & 76.18 & 62.86 \\
\hline 40.00 & 65.04 & 83.83 & 86.30 & 86.49 & 86.56 & 86.55 & 86.41 & 86.04 & 75.68 & 62.75 \\
\hline 41.00 & 65.05 & 83.99 & 86.46 & 86.65 & 86.72 & 86.72 & 86.57 & 86.20 & 75.83 & 62.77 \\
\hline 42.00 & 64.70 & 82.60 & 84.93 & 85.13 & 85.20 & 85.19 & 85.05 & 84.68 & 74.94 & 62.47 \\
\hline 43.00 & 64.66 & 82.73 & 85.06 & 85.26 & 85.33 & 85.33 & 85.18 & 84.81 & 75.05 & 62.45 \\
\hline 44.00 & 61.30 & 62.73 & 63.14 & 63.32 & 63.41 & 63.40 & 63.23 & 62.96 & 62.37 & 60.30 \\
\hline 45.00 & 60.65 & 62.32 & 62.60 & 62.78 & 62.86 & 62.85 & 62.68 & 62.47 & 62.03 & 59.83 \\
\hline
\end{tabular}

